\title{
LOG CANONICAL THRESHOLDS OF SMOOTH FANO THREEFOLDS
}

\author{
IVAN CHELTSOV AND CONSTANTIN SHRAMOV
}

WITH AN APPENDIX BY JEAN-PIERRE DEMAILLY

ABSTRACT. We compute global log canonical thresholds of some smooth Fano threefolds.

\section{Contents}

1. Introduction

2. Preliminaries

3. The Mukai-Umemura threefold

4. Cubic surfaces

5. Del Pezzo surfaces

6. Toric varieties

7. Del Pezzo threefolds

8. Fano threefolds with $\rho=2$

9. Fano threefolds with $\rho=3$

10. Fano threefolds with $\rho \geqslant 4$

11. Upper bounds

Appendix A. By Jean-Pierre Demailly. On Tian's invariant and log canonical thresholds 100 Appendix B. The Big Table

\section{INTRODUCTION}

The multiplicity of a polynomial $\phi \in \mathbb{C}\left[z_{1}, \ldots, z_{n}\right]$ in the origin $O \in \mathbb{C}^{n}$ is the number

$$
\min \left\{m \in \mathbb{Z}_{\geqslant 0} \mid \frac{\partial^{m} \phi\left(z_{1}, \ldots, z_{n}\right)}{\partial^{m_{1}} z_{1} \partial^{m_{2}} z_{2} \ldots \partial^{m_{n}} z_{n}}(O) \neq 0\right\} \in \mathbb{Z}_{\geqslant 0} \cup\{+\infty\} .
$$

There is a similar but more subtle invariant

$c_{0}(\phi)=\sup \left\{\varepsilon \in \mathbb{Q} \mid\right.$ the function $\frac{1}{|\phi|^{2 \varepsilon}}$ is locally integrable near $\left.O \in \mathbb{C}^{n}\right\} \in \mathbb{Q} \geqslant 0 \cup\{+\infty\}$,

which is called the complex singularity exponent of the polynomial $\phi$ at the point $O$.

Example 1.1. Suppose that $n=2$, and $\phi=0$ defines an irreducible curve in $\mathbb{C}^{2}$. Then

$$
c_{0}(\phi)=\min \left(1, \frac{1}{m}+\frac{1}{n}\right)
$$

by [90, where $(m, n)$ is the first pair of Puiseux exponents of $\phi$. On the other hand, the equality

$$
c_{0}\left(z_{1}^{n_{1}} z_{2}^{n_{2}}\left(z_{1}^{k m_{1}}+z_{2}^{k m_{2}}\right)\right)=\min \left(\frac{1}{n_{1}}, \frac{1}{n_{2}}, \frac{\frac{1}{m_{1}}+\frac{1}{m_{2}}}{k+\frac{n_{1}}{m_{1}}+\frac{n_{2}}{m_{2}}}\right)
$$

holds (see [109]), where $n_{1}, n_{2}, m_{1}, m_{2}, k$ are non-negative integers.

The first author was supported by the grants NSF DMS-0701465 and EPSRC EP/E048412/1, the second author was supported by the grants RFFI No. 08-01-00395-a, N.Sh.-1987.2008.1 and EPSRC EP/E048412/1. 
Example 1.2. Let $m_{1}, \ldots, m_{n}$ be positive integers. Then

$$
\min \left(1, \sum_{i=1}^{n} \frac{1}{m_{i}}\right)=c_{0}\left(\sum_{i=1}^{n} z_{i}^{m_{i}}\right) \geqslant c_{0}\left(\prod_{i=1}^{n} z_{i}^{m_{i}}\right)=\min \left(\frac{1}{m_{1}}, \frac{1}{m_{2}}, \ldots, \frac{1}{m_{n}}\right) .
$$

The set of complex singularity exponents has interesting properties. Put

$$
\mathcal{H}_{n}=\left\{c_{0}(\phi) \mid \phi \in \mathbb{C}\left[z_{1}, \ldots, z_{n}\right]\right\} \subset \mathbb{Q} \cup\{+\infty\}
$$

which implies that $\mathcal{T}_{n} \subset[0,1] \cup\{+\infty\}$. Then

- the set $\mathcal{H}_{n}$ is closed in $\mathbb{R} \cup\{+\infty\}$ (see [60]),

- we expect that $\mathcal{H}_{n}$ satisfies ascending chain condition (ACC) for every $n$ (see [106]),

- the set $\mathcal{H}_{n}$ satisfies ACC for $n \leqslant 3$ (see [169], [1], [109], [141], [58]),

- it follows from [60] that the following assertions are equivalent:

- the set $\mathcal{H}_{n}$ satisfies ACC for every $n \in \mathbb{Z}_{>0}$;

- for every $n \in \mathbb{Z}_{>0}$, there is $\delta_{n} \in(0,1)$ such that $\mathcal{H}_{n} \cap\left(\delta_{n}, 1\right)=\varnothing$;

- it follows from [106] that $\mathcal{H}_{n-1} \subset \mathcal{H}_{n}$ and

$$
\mathcal{H}_{n-1} \backslash\{1,+\infty\} \subseteq \partial \mathcal{H}_{n} \subseteq \mathcal{H}_{n-1} \backslash\{+\infty\},
$$

where $\partial \mathcal{H}_{n}$ is the set of all accumulation points of $\mathcal{H}_{n}$,

- it follows from [109] and [110] that the set $\mathcal{H}_{2}$ is the union

$$
\left\{\frac{2}{m} \mid 2 \leqslant m \in \mathbb{Z}_{>0}\right\} \bigcup\left\{\frac{m_{1}+m_{2}}{k m_{1} m_{2}+n_{1} m_{2}+n_{2} m_{1}} \begin{array}{c}
-k m_{1}<n_{1}-n_{2}<k m_{2}, \\
\operatorname{gcd}\left(m_{1}, m_{2}\right)=1, \\
k, m_{1}, m_{2}, n_{1}, n_{2} \in \mathbb{Z}_{\geqslant 0}
\end{array}\right\} \bigcup\{0,+\infty\},
$$

which implies that $\partial \mathcal{H}_{2}=\mathcal{H}_{1} \backslash\{1,+\infty\}$, where $\mathcal{H}_{1}=\left\{1 / n \mid n \in \mathbb{Z}_{\geqslant 0}\right\} \cup\{0\}$,

- it follows from [110] that the intersection $\mathcal{H}_{3} \cap\left[\frac{5}{6}, 1\right)$ is the union

$$
\left\{\frac{5}{6}+\frac{1}{m} \mid m \geqslant 6\right\} \bigcup\left\{\frac{5}{6}+\frac{2}{3 m} \mid m \geqslant 4\right\} \bigcup\left\{\frac{5}{6}+\frac{4}{9 m+6} \mid m \geqslant 2\right\} \bigcup\left\{\frac{19}{20}, \frac{15}{16}, \frac{12}{13}, \frac{25}{28}, \frac{15}{17}, \frac{5}{6}\right\},
$$

where $m \in \mathbb{Z}_{>0}$, which implies that $5 / 6$ is the largest accumulation point of $\mathcal{H}_{3}$ (cf. [145], [146]),

- it follows from [124] that $\partial \mathcal{H}_{3}=\mathcal{H}_{2} \backslash\{1,+\infty\}$ (cf. [106]),

- it follows from [104] that $41 / 42$ is the maximal element of the set $\mathcal{H}_{3} \cap[0,1)$.

Remark 1.3. For a non-constant $\phi$, the complex singularity exponent $c_{0}(\phi)$ coincides with the absolute value of the biggest root of the Bernstein-Sato polynomial of $\phi$ (see [10], [105]).

Let $X$ be a variety 1 with at most $\log$ canonical singularities (see [102]), let $Z \subseteq X$ be a closed subvariety, and let $D$ be an effective $\mathbb{Q}$-Cartier $\mathbb{Q}$-divisor on the variety $X$. Then the number

$$
\operatorname{lct}_{Z}(X, D)=\sup \{\lambda \in \mathbb{Q} \mid \text { the } \log \text { pair }(X, \lambda D) \text { is } \log \text { canonical along } Z\} \in \mathbb{Q} \cup\{+\infty\}
$$

is called a $\log$ canonical threshold of the divisor $D$ along $Z$. It follows from [105] that

$$
\operatorname{lct}_{O}\left(\mathbb{C}^{n},(\phi=0)\right)=c_{0}(\phi),
$$

so that $\operatorname{lct}_{Z}(X, D)$ is an algebraic counterpart of the number $c_{0}(\phi)$. One has $\operatorname{lct}_{X}(X, D)=\inf \left\{\operatorname{lct}_{P}(X, D) \mid P \in X\right\}=\sup \{\lambda \in \mathbb{Q} \mid$ the log pair $(X, \lambda D)$ is log canonical $\}$, and, for simplicity, we put $\operatorname{lct}(X, D)=\operatorname{lct}_{X}(X, D)$.

\footnotetext{
${ }^{1}$ All varieties are assumed to be complex, algebraic, projective and normal if the opposite is not stated explicitly.
} 
Example 1.4. Suppose that $X=\mathbb{P}^{2}$ and $D \in\left|\mathcal{O}_{\mathbb{P}^{2}}(3)\right|$. Then

$$
\operatorname{lct}(X, D)=\left\{\begin{array}{l}
1 \text { if } D \text { is a smooth curve, } \\
1 \text { if } D \text { is a curve with ordinary double points, } \\
5 / 6 \text { if } D \text { is a curve with one cuspidal point, } \\
3 / 4 \text { if } D \text { consists of a conic and a line that are tangent, } \\
2 / 3 \text { if } D \text { consists of three lines intersecting at one point, } \\
1 / 2 \text { if } \operatorname{Supp}(D) \text { consists of two lines, } \\
1 / 3 \text { if } \operatorname{Supp}(D) \text { consists of one line. }
\end{array}\right.
$$

Example 1.5. Suppose that $X=\mathbb{P}^{2}$ and $D \in\left|\mathcal{O}_{\mathbb{P}^{2}}(d)\right|$ for $d \geqslant 3$. The papers [103] and [112] show that the curve $D$ is semistable (stable, respectively) if $\operatorname{lct}(X, D) \geqslant 3 / d(>3 / d$, respectively).

The set of log canonical thresholds of Weil divisors has interesting properties, which are similar to the properties of the set $\mathcal{H}_{n}$ (cf. [8]). Put

$$
\mathcal{T}_{n}=\left\{\begin{array}{l|l}
\operatorname{lct}(X, D) & \begin{array}{l}
\text { the variety } X \text { has at most log canonical singularities, } \\
\operatorname{dim}(X)=n \text { and } D \text { is effective } \mathbb{Q} \text {-Cartier Weil divisor }
\end{array}
\end{array}\right\} \subset \mathbb{Q} \cup\{+\infty\},
$$

which implies that $\mathcal{T}_{n} \subset[0,1] \cup\{+\infty\}$. Then

- the set $\mathcal{T}_{n}$ satisfies ACC for $n \leqslant 3$ (see [169, [1, [109], [141, [58]),

- we expect that $\mathcal{T}_{n}$ satisfies ACC for every $n$ (see [105, Conjecture 8.8]),

- it follows from [105, Proposition 8.8] that that $\mathcal{T}_{n-1} \subset \mathcal{T}_{n}$ and

$$
\mathcal{T}_{n-1} \backslash\{1,+\infty\} \subseteq \partial \mathcal{T}_{n}
$$

where $\partial \mathcal{T}_{n}$ is the set of all accumulation points of $\mathcal{T}_{n}$,

- it follows from [169] and [109] that $\partial \mathcal{T}_{2}=\mathcal{T}_{1} \backslash\{1,+\infty\}$, where $\mathcal{T}_{1}=\left\{1 / n \mid n \in \mathbb{Z}_{\geqslant 0}\right\} \cup\{0\}$,

- it follows from [145] and [146] that

$$
\partial \mathcal{T}_{3} \cap\left[\frac{1}{2}, 1\right]=\mathcal{T}_{2} \cap\left[\frac{1}{2}, 1\right]=\left\{\frac{1}{2}+\frac{1}{n} \mid 3 \leqslant n \in \mathbb{Z}_{>0}\right\},
$$

which implies that $5 / 6$ is the largest accumulation point of $\mathcal{T}_{3}$ (cf. [110]),

- it follows from 124 that $\partial \mathcal{T}_{3}=\mathcal{T}_{2} \backslash\{1,+\infty\}$ (cf. [106]),

- it follows from [104 that $41 / 42$ is is the maximal element of the set $\mathcal{T}_{3} \cap[0,1)$.

Remark 1.6. If $X$ is smooth, and $D$ is a Weil divisor, then it follows from [131] that

$$
\operatorname{lct}(X, D)=\operatorname{dim}(X)-\sup \left\{\frac{\operatorname{dim}\left(D_{m}\right)}{m+1} \mid m \in \mathbb{Z}_{\geqslant 0}\right\},
$$

where $D_{m}$ is the $m$-th jet scheme of the divisor $D$ (see [131]).

Suppose that $X$ is a Fano variety with at most log terminal singularities (see [98]).

Definition 1.7. Global $\log$ canonical threshold of the Fano variety $X$ is the number

$$
\operatorname{lct}(X)=\inf \left\{\operatorname{lct}(X, D) \mid D \text { is an effective } \mathbb{Q} \text {-divisor on } X \text { such that } D \sim_{\mathbb{Q}}-K_{X}\right\} \geqslant 0 \text {. }
$$

Remark 1.8. To define the number $\operatorname{lct}(X) \in \mathbb{R}$, we only need to assume that

$$
\left|-n K_{X}\right| \neq \varnothing
$$

for some $n \gg 0$. This property is shared by many varieties (toric varieties, weak Fano varieties), but all the currently known applications are related to the case when $-K_{X}$ is ample.

The number $\operatorname{lct}(X)$ is an algebraic counterpart of the $\alpha$-invariant introduced in [179]. One has

$$
\operatorname{lct}(X)=\sup \left\{\begin{array}{l|l}
\varepsilon \in \mathbb{Q} & \begin{array}{l}
\text { the log pair }\left(X, \frac{\varepsilon}{n} D\right) \text { is log canonical for } \\
\text { every divisor } D \in\left|-n K_{X}\right| \text { and all } n \in \mathbb{Z}_{>0}
\end{array}
\end{array}\right\} .
$$


Recall that every Fano variety $X$ is rationally connected (see [170, [193]). Thus, the group $\operatorname{Pic}(X)$ is torsion free. Then

$$
\operatorname{lct}(X)=\sup \left\{\begin{array}{l|l}
\lambda \in \mathbb{Q} & \begin{array}{l}
\text { the log pair }(X, \lambda D) \text { is log canonical } \\
\text { for every effective } \mathbb{Q} \text {-divisor } D \sim_{\mathbb{Q}}-K_{X}
\end{array}
\end{array}\right\} .
$$

Example 1.9. Let $X$ be a smooth hypersurface in $\mathbb{P}^{n}$ of degree $m<n$. Then

$$
\operatorname{lct}(X)=\frac{1}{n+1-m}
$$

as shown in 20] (see Corollary 2.16). In particular, the equality $\operatorname{lct}\left(\mathbb{P}^{n}\right)=1 /(n+1)$ holds.

Example 1.10. Let $X$ be a rational homogeneous space such that $-K_{X} \sim r D$ and

$$
\operatorname{Pic}(X)=\mathbb{Z}[D]
$$

where $D$ is an ample divisor and $r \in \mathbb{Z}_{>0}$. Then $\operatorname{lct}(X)=1 / r$ (see [78, [79]).

Example 1.11. Let $X$ be a general intersection of hypersurfaces $F_{1}, \ldots, F_{k} \subset \mathbb{P}^{n}$ such that

$$
\sum_{i=1}^{k} \operatorname{deg}\left(F_{i}\right)=n \geqslant 5 k+1 \geqslant 11
$$

where $\operatorname{deg}\left(F_{k}\right) \geqslant \ldots \geqslant \operatorname{deg}\left(F_{1}\right) \geqslant 2$ and $\operatorname{deg}\left(F_{k}\right) \geqslant 8$. Then $\operatorname{lct}(X)=1$ (see [158]).

In general, the number $\operatorname{lct}(X)$ depends on small deformations of the variety $X$.

Example 1.12. Let $X$ be a smooth hypersurface in $\mathbb{P}(1,1,1,1,3)$ of degree 6 . Then

$$
\operatorname{lct}(X) \in\left\{\frac{5}{6}, \frac{43}{50}, \frac{13}{15}, \frac{33}{38}, \frac{7}{8}, \frac{33}{38}, \frac{8}{9}, \frac{9}{10}, \frac{11}{12}, \frac{13}{14}, \frac{15}{16}, \frac{17}{18}, \frac{19}{20}, \frac{21}{22}, \frac{29}{30}, 1\right\}
$$

by [157] and [37, and all these values are attained.

Example 1.13. Let $X$ be a smooth hypersurface in $\mathbb{P}\left(1^{n+1}, n\right)$ of degree $2 n$. The inequalities

$$
1 \geqslant \operatorname{lct}(X) \geqslant \frac{2 n-1}{2 n}
$$

hold (see [37]). But the equality $\operatorname{lct}(X)=1$ holds if $X$ is general and $n \geqslant 3$.

Example 1.14. Let $X$ be a smooth hypersurface in $\mathbb{P}^{n}$ of degree $n \geqslant 2$. Then the inequalities

$$
1 \geqslant \operatorname{lct}(X) \geqslant \frac{n-1}{n}
$$

hold (see [20]). Then it follows from [157] and [37] that

$$
\operatorname{lct}(X) \geqslant\left\{\begin{array}{l}
1 \text { if } n \geqslant 6, \\
22 / 25 \text { if } n=5, \\
16 / 21 \text { if } n=4, \\
3 / 4 \text { if } n=3,
\end{array}\right.
$$

whenver $X$ is general. But $\operatorname{lct}(X)=1-1 / n$ if $X$ contains a cone of dimension $n-2$.

Example 1.15. Let $X$ be a quasismooth hypersurface in $\mathbb{P}\left(1, a_{1}, a_{2}, a_{3}, a_{4}\right)$ of degree $\sum_{i=1}^{4} a_{i}$ such that $X$ has at most terminal singularities (see [102]), where $a_{1} \leqslant a_{2} \leqslant a_{3} \leqslant a_{4}$. Then

$$
-\left.K_{X} \sim \mathcal{O}_{\mathbb{P}\left(1, a_{1}, a_{2}, a_{3}, a_{4}\right)}(1)\right|_{X}
$$

and there are 95 possibilities for the quadruple $\left(a_{1}, a_{2}, a_{3}, a_{4}\right)$ (see [89], 82]). Then

$$
1 \geqslant \operatorname{lct}(X) \geqslant\left\{\begin{array}{l}
16 / 21 \text { if } a_{1}=a_{2}=a_{3}=a_{4}=1, \\
7 / 9 \text { if }\left(a_{1}, a_{2}, a_{3}, a_{4}\right)=(1,1,1,2), \\
4 / 5 \text { if }\left(a_{1}, a_{2}, a_{3}, a_{4}\right)=(1,1,2,2), \\
6 / 7 \text { if }\left(a_{1}, a_{2}, a_{3}, a_{4}\right)=(1,1,2,3), \\
1 \text { in the remaining cases, }
\end{array}\right.
$$


if $X$ is general (see [27], 37, 29], 30]). The global log canonical threshold of the hypersurface

$$
w^{2}=t^{3}+z^{9}+y^{18}+x^{18} \subset \mathbb{P}(1,1,2,6,9) \cong \operatorname{Proj}(\mathbb{C}[x, y, z, t, w])
$$

is equal to $17 / 18$ (see [27]), where $\operatorname{wt}(x)=\operatorname{wt}(y)=1, \operatorname{wt}(z)=2, \operatorname{wt}(t)=6, \operatorname{wt}(w)=9$.

Example 1.16. It follows from Lemma 6.1 that

$$
\operatorname{lct}\left(\mathbb{P}\left(a_{0}, a_{1}, \ldots, a_{n}\right)\right)=\frac{a_{0}}{\sum_{i=0}^{n} a_{i}},
$$

where $\mathbb{P}\left(a_{0}, a_{1}, \ldots, a_{n}\right)$ is well-formed (see [89]), and $a_{0} \leqslant a_{1} \leqslant \ldots \leqslant a_{n}$.

Example 1.17. Let $X$ be a smooth hypersurface in $\mathbb{P}\left(1^{n+1}, d\right)$ of degree $2 d$. Then

$$
\operatorname{lct}(X)=\frac{1}{n+1-d}
$$

in the case when the inequalities $2 \leqslant d \leqslant n-1$ hold (see Proposition 20 in [28]).

Example 1.18. Let $X$ be smooth surface del Pezzo. It follows from [31] that

$$
\operatorname{lct}(X)=\left\{\begin{array}{l}
1 \text { if } K_{X}^{2}=1 \text { and }\left|-K_{X}\right| \text { contains no cuspidal curves, } \\
5 / 6 \text { if } K_{X}^{2}=1 \text { and }\left|-K_{X}\right| \text { contains a cuspidal curve, } \\
5 / 6 \text { if } K_{X}^{2}=2 \text { and }\left|-K_{X}\right| \text { contains no tacnodal curves, } \\
3 / 4 \text { if } K_{X}^{2}=2 \text { and }\left|-K_{X}\right| \text { contains a tacnodal curve, } \\
3 / 4 \text { if } X \text { is a cubic in } \mathbb{P}^{3} \text { with no Eckardt points, } \\
2 / 3 \text { if either } X \text { is a cubic in } \mathbb{P}^{3} \text { with an Eckardt point, or } K_{X}^{2}=4 \\
1 / 2 \text { if } X \cong \mathbb{P}^{1} \times \mathbb{P}^{1} \text { or } K_{X}^{2} \in\{5,6\} \\
1 / 3 \text { in the remaining cases. }
\end{array}\right.
$$

It would be interesting to compute global log canonical thresholds of del Pezzo surfaces with at most canonical singularities that are of Picard rank one, which has been classified in [62]

Example 1.19. Let $X$ be a singular cubic surface in $\mathbb{P}^{3}$ such that $X$ has at most canonical singularities. The singularities of the surface $X$ are classified in [16]. It follows from [32] that

$$
\operatorname{lct}(X)=\left\{\begin{array}{l}
2 / 3 \text { if } \operatorname{Sing}(X)=\left\{\mathbb{A}_{1}\right\} \\
1 / 3 \text { if } \operatorname{Sing}(X) \supseteq\left\{\mathbb{A}_{4}\right\} \\
1 / 3 \text { if } \operatorname{Sing}(X)=\left\{\mathbb{D}_{4}\right\} \\
1 / 3 \text { if } \operatorname{Sing}(X) \supseteq\left\{\mathbb{A}_{2}, \mathbb{A}_{2}\right\} \\
1 / 4 \text { if } \operatorname{Sing}(X) \supseteq\left\{\mathbb{A}_{5}\right\} \\
1 / 4 \text { if } \operatorname{Sing}(X)=\left\{\mathbb{D}_{5}\right\} \\
1 / 6 \text { if } \operatorname{Sing}(X)=\left\{\mathbb{E}_{6}\right\} \\
1 / 2 \text { in the remaining cases. }
\end{array}\right.
$$

It is unknown whether $\operatorname{lct}(X) \in \mathbb{Q}$ or not? (cf. Question 1 in [181]).

Conjecture 1.20. There is an effective $\mathbb{Q}$-divisor $D \sim_{\mathbb{Q}}-K_{X}$ on the variety $X$ such that

$$
\operatorname{lct}(X)=\operatorname{lct}(X, D) \in \mathbb{Q} \text {. }
$$

Let $G \subset \operatorname{Aut}(X)$ be an arbitrary subgroup.

Definition 1.21. Global $G$-invariant $\log$ canonical threshold of the Fano variety $X$ is $\operatorname{lct}(X, G)=\sup \left\{\begin{array}{l|l}\varepsilon \in \mathbb{Q} & \begin{array}{l}\text { the log pair }\left(X, \frac{\varepsilon}{n} \mathcal{D}\right) \text { has log canonical singularities for every } \\ G \text {-invariant linear system } \mathcal{D} \subset\left|-n K_{X}\right| \text { and every } n \in \mathbb{Z}_{>0}\end{array}\end{array}\right\}$.

\footnotetext{
${ }^{2}$ It is even unknown whether $\operatorname{lct}(X) \in \mathbb{Q}$ or not if $X$ is a del Pezzo surfaces with log terminal singularities.
} 
Remark 1.22. To define the threshold $\operatorname{lct}(X, G) \in \mathbb{R} \cup\{+\infty\}$, we only need to assume that

$$
\left|-n K_{X}\right| \neq \varnothing
$$

for some $n \gg 0$. But all known applications require $-K_{X}$ to be ample, and $G$ to be compact.

In the case when the Fano variety $X$ is smooth and $G$ is compact, the equality

$$
\operatorname{lct}(X, G)=\alpha_{G}(X),
$$

holds (see Appendix $\overline{\mathrm{A}}$ ), where $\alpha_{G}(X)$ is the $\alpha$-invariant introduced in [179]. It is clear that

$$
\operatorname{lct}(X, G)=\sup \left\{\begin{array}{l|l}
\lambda \in \mathbb{Q} & \begin{array}{l}
\text { the log pair }(X, \lambda D) \text { has log canonical singularities } \\
\text { for every } G \text {-invariant effective } \mathbb{Q} \text {-divisor } D \sim_{\mathbb{Q}}-K_{X}
\end{array}
\end{array}\right\}
$$

in the case when $|G|<+\infty$. Note that $0 \leqslant \operatorname{lct}(X) \leqslant \operatorname{lct}(X, G) \in \mathbb{R} \cup\{+\infty\}$.

Example 1.23. The simple group $\operatorname{PGL}\left(2, \mathrm{~F}_{7}\right)$ is a group of automorphisms of the quartic

$$
x^{3} y+y^{3} z+z^{3} x=0 \subset \mathbb{P}^{2} \cong \operatorname{Proj}(\mathbb{C}[x, y, z]),
$$

which induces an embedding $\operatorname{PGL}\left(2, \mathrm{~F}_{7}\right) \subset \operatorname{Aut}\left(\mathbb{P}^{2}\right)$. Then $\operatorname{lct}\left(\mathbb{P}^{2}, \operatorname{PGL}\left(2, \mathrm{~F}_{7}\right)\right)=4 / 3$ (see [31]).

Example 1.24. Let $X$ be a smooth del Pezzo surface such that $K_{X}^{2}=5$. Then

- the isomorphism $\operatorname{Aut}(X) \cong \mathrm{S}_{5}$ holds (see [161]),

- the equalities $\operatorname{lct}\left(X, \mathrm{~S}_{5}\right)=\operatorname{lct}\left(X, \mathrm{~A}_{5}\right)=2$ hold (see [31]).

Example 1.25. Let $X$ be the cubic surface in $\mathbb{P}^{3}$ given by the equation

$$
x^{3}+y^{3}+z^{3}+t^{3}=0 \subset \mathbb{P}^{3} \cong \operatorname{Proj}(\mathbb{C}[x, y, z, t]),
$$

and let $G=\operatorname{Aut}(X) \cong \mathbb{Z}_{3}^{3} \rtimes \mathrm{S}_{4}$. Then $\operatorname{lct}(X, G)=4$ by [31].

The following result was proved in [179, [132, [49] (see Appendix A).

Theorem 1.26. Suppose that $X$ has at most quotient singularities, and the inequality

$$
\operatorname{lct}(X, G)>\frac{\operatorname{dim}(X)}{\operatorname{dim}(X)+1}
$$

holds. Then $X$ admits an orbifold Kähler-Einstein metric.

Let us show how to apply Theorem 1.26 (cf. Examples 1.13, 1.14, 1.15).

Example 1.27. Let $X$ be a quasismooth hypersurface in $\mathbb{P}\left(a_{0}, a_{1}, a_{2}, a_{3}\right)$ of degree $\sum_{i=0}^{3} a_{i}-1$, where $a_{0} \leqslant a_{1} \leqslant a_{2} \leqslant a_{3}$. Then it follows from [49], [81, [13], [2] (cf. [14], [15]) that

- either the surface $X$ is smooth, which implies that

$$
\left(a_{0}, a_{1}, a_{2}, a_{3}\right) \in\{(1,1,1,1),(1,1,1,2),(1,1,2,3)\},
$$

and all possible values of lct $(X)$ are contained in Example 1.18,

- or $\left(a_{0}, a_{1}, a_{2}, a_{3}\right)=(2,2 n+1,2 n+1,4 n+1)$ and $\operatorname{lct}(X)=1$, where $n \in \mathbb{Z}_{\geqslant 2}$,

- or we have the following sporadic possibilities:

$-\left(a_{0}, a_{1}, a_{2}, a_{3}\right)=(2,3,3,5)$ and $\operatorname{lct}(X) \geqslant 33 / 38$

$-\left(a_{0}, a_{1}, a_{2}, a_{3}\right)=(1,2,3,5)$ and $\operatorname{lct}(X)>2 / 3$;

- $\left(a_{0}, a_{1}, a_{2}, a_{3}\right)=(1,3,5,7)$ and $\operatorname{lct}(X)>2 / 3$ if $X \subset \mathbb{P}(1,3,5,7)$ is general;

$-\left(a_{0}, a_{1}, a_{2}, a_{3}\right)=(1,3,5,8)$ and $\operatorname{lct}(X) \geqslant 11 / 16$ if $X \subset \mathbb{P}(1,3,5,8)$ is general;

- the inequality $\operatorname{lct}(X)>1$ holds and

$$
\left(a_{0}, a_{1}, a_{2}, a_{3}\right) \in\left\{\begin{array}{l}
(2,3,5,9),(3,3,5,5),(3,5,7,11),(3,5,7,14),(3,5,11,18) \\
(5,14,17,21),(5,19,27,31),(5,19,27,50),(7,11,27,37) \\
(7,11,27,44),(9,15,17,20),(9,15,23,23),(11,29,39,49) \\
(11,49,69,128),(13,23,35,57),(13,35,81,128)
\end{array}\right\} .
$$


Example 1.28. Let $X$ be a quasismooth hypersurface in $\mathbb{P}\left(a_{0}, \ldots, a_{4}\right)$ of degree $\sum_{i=0}^{4} a_{i}-1$, where $a_{0} \leqslant a_{1} \leqslant a_{2} \leqslant a_{3} \leqslant a_{4}$. Then it follows from [82] that

- the inequality $\operatorname{lct}(X)>3 / 4$ holds for at least 1936 quintuples $\left(a_{0}, a_{1}, a_{2}, a_{3}, a_{4}\right)$,

- the inequality $\operatorname{lct}(X) \geqslant 1$ holds for at least 1605 quintuples $\left(a_{0}, a_{1}, a_{2}, a_{3}, a_{4}\right)$.

Example 1.29. Let $X$ be one of the following smooth Fano varieties:

- a Fermat hypersurface in $\mathbb{P}^{n}$ of degree $n / 2 \leqslant d \leqslant n$ (cf. Example 1.25);

- a general complete intersection of three quadrics in $\mathbb{P}^{6}$ that is given by

$$
\sum_{i=0}^{6} x_{i}^{2}=\sum_{i=0}^{6} \lambda_{i} x_{i}^{2}=\sum_{i=0}^{6} \mu_{i} x_{i}^{2}=0 \subseteq \mathbb{P}^{6} \cong \operatorname{Proj}\left(\mathbb{C}\left[x_{0}, \ldots, x_{6}\right]\right),
$$

where $\lambda_{i}$ and $\mu_{i}$ are complex numbers;

- a smooth complete intersection of two quadrics in $\mathbb{P}^{5}$ that is given by

$$
\sum_{i=0}^{5} x_{i}^{2}=\sum_{i=0}^{5} \zeta^{i} x_{i}^{2}=0 \subseteq \mathbb{P}^{5} \cong \operatorname{Proj}\left(\mathbb{C}\left[x_{0}, \ldots, x_{5}\right]\right),
$$

where $\zeta$ is a primitive sixth root of unity;

- a smooth complete intersection of a quadric and a cubic in $\mathbb{P}^{5}$ that is given by

$$
\sum_{i=0}^{5} x_{i}^{3}=\sum_{i=0}^{5} \zeta^{i} x_{i}^{2}=0 \subseteq \mathbb{P}^{5} \cong \operatorname{Proj}\left(\mathbb{C}\left[x_{0}, \ldots, x_{5}\right]\right),
$$

where $\zeta$ is a nontrivial cube root of unity;

- a hypersurface in $\mathbb{P}\left(1^{n+1}, q\right)$ of degree $p q$ that is given by the equation

$$
w^{p}=\sum_{i=0}^{5} x_{i}^{p q} \subseteq \mathbb{P}\left(1^{n+1}, q\right) \cong \operatorname{Proj}\left(\mathbb{C}\left[x_{0}, \ldots, x_{n}, w\right]\right),
$$

such that $p q-q \leqslant n$, where $\operatorname{wt}\left(x_{0}\right)=\ldots=\operatorname{wt}\left(x_{n}\right)=1, \operatorname{wt}(w)=q \in \mathbb{Z}_{>0}$ and $p \in \mathbb{Z}_{>0} ;$ and let $G=\operatorname{Aut}(X)$. Then $G$ is finite, and the inequality $\operatorname{lct}(X, G) \geqslant 1$ holds (see [179], [132]).

Example 1.30. Let $X$ be a blow up of $\mathbb{P}^{3}$ along a disjoint union of two lines, let $G$ be a maximal compact subgroup in $\operatorname{Aut}(X)$. Then the inequality $\operatorname{lct}(X, G) \geqslant 1$ holds by [132] (cf. Lemma 9.26).

If a variety with quotient singularities admits an orbifold Kähler-Einstein metric, then

- either its canonical divisor is numerically trivial;

- or its canonical divisor is ample (variety of general type);

- or its canonical divisor is antiample (Fano variety).

Remark 1.31. Every variety with at most quotient singularities that has numerically trivial or ample canonical divisor always admits an orbifold Kähler-Einstein metric (see [5], [190], [191]).

There are several known obstructions for the Fano variety $X$ to admit a Kähler-Einstein metric. For example, if the variety $X$ is smooth, then it does not admit a Kähler-Einstein metric if

- either the group $\operatorname{Aut}(X)$ is not reductive (see [122]),

- or the tangent bundle of $X$ is not polystable with respect to $-K_{X}$ (see [114]),

- or the Futaki character of holomorphic vector fields on $X$ does not vanish (see [68]),

- or the pair $\left(X,-K_{X}\right)$ is not $K$-semistable (see [182, [53], [54, [162], [163]).

Example 1.32. The following varieties admit no Kähler-Einstein metrics:

- a blow up of $\mathbb{P}^{2}$ in one or two points (see [122]),

- a smooth Fano threefold $\mathbb{P}\left(\mathcal{O}_{\mathbb{P}^{2}} \oplus \mathcal{O}_{\mathbb{P}^{2}}(1)\right)$ (see [176]),

- a smooth Fano fourfold

$$
\mathbb{P}\left(\alpha^{*}\left(\mathcal{O}_{\mathbb{P}^{1}}(1)\right) \oplus \beta^{*}\left(\mathcal{O}_{\mathbb{P}^{2}}(1)\right)\right),
$$

where $\alpha: \mathbb{P}^{1} \times \mathbb{P}^{2} \rightarrow \mathbb{P}^{1}$ and $\beta: \mathbb{P}^{1} \times \mathbb{P}^{2} \rightarrow \mathbb{P}^{2}$ are natural projections (see [68]). 
Example 1.33. Let $X$ be a smooth Fano threefold such that

$$
\operatorname{Pic}(X)=\mathbb{Z}\left[-K_{X}\right]
$$

and $-K_{X}^{3}=22$. Then $\operatorname{lct}(X) \leqslant 2 / 3$ by Lemma 11.3 (see Section 3 ). But

- the tangent bundle of the threefold $X$ is stable (see [176]),

- the group $\operatorname{Aut}(X)$ is trivial if the threefold $X$ is general (see [167]),

- there is $X$ such that $\operatorname{Aut}(X)=\{1\}$ and $X$ admits no Kähler-Einstein metrics (see [182]);

- there is $X$, whose group of automorphisms $\operatorname{Aut}(X)$ is not non-reductive (see [142]);

- if $\operatorname{Aut}(X) \cong \operatorname{PSL}(2, \mathbb{C})$, then $X$ has a Kähler-Einstein metric (see [55] and Remark 3.2).

Recently new obstruction for the existence of orbifold Kähler-Einstein metrics on Fano varieties with at most quotient singularities have been found (see [69], [175, [11]).

Example 1.34. Let $X$ be a quasismooth hypersurface in $\mathbb{P}\left(a_{0}, \ldots, a_{n}\right)$ of degree $d<\sum_{i=0}^{n} a_{i}$, where $a_{0} \leqslant \ldots, \leqslant a_{n}$ and $\mathbb{P}\left(a_{0}, \ldots, a_{n}\right)$ is well-formed (see [89]). Then $X$ is a Fano variety. If

$$
\sum_{i=0}^{n} a_{i}>d+n a_{0},
$$

then $X$ admits no orbifold Kähler-Einstein metric (see [69], [175]).

The problem of existence of Kähler-Einstein metrics on smooth toric Fano varieties is completely solved. Namely, the following result holds (see [115], 7], [187, [133]).

Theorem 1.35. If $X$ is smooth and toric, then the following conditions are equivalent:

- the variety $X$ admits a Kähler-Einstein metric;

- the Futaki character of holomorphic vector field of $X$ vanishes;

- the baricenter of the reflexive polytope of $X$ is zero.

It should be pointed out that the assertion of Theorem 1.26 gives only a sufficient condition for the existence of a Kähler-Einstein metric on $X$ (cf. [119], [182]).

Example 1.36. Let $X$ be a general cubic surface in $\mathbb{P}^{3}$ that has an Eckardt point (see Definition 4.1). Then $\operatorname{Aut}(X) \cong \mathbb{Z}_{2}$ (see [52]) and

$$
\operatorname{lct}(X, \operatorname{Aut}(X))=\operatorname{lct}(X)=\frac{2}{3}
$$

by [31. But every smooth del Pezzo surface that has a reductive automorphism groups admits a Kähler-Einstein metric (see [183], 180]).

Example 1.37. Let $X$ be a general hypersurface in $\mathbb{P}\left(1^{5}, 3\right)$ of degree 6 . Then $\operatorname{Aut}(X) \cong \mathbb{Z}_{2}$ (see [123]) and

$$
\operatorname{lct}(X, \operatorname{Aut}(X))=\operatorname{lct}(X)=\frac{1}{2}
$$

by [28]. But $X$ admit a Kähler-Einstein metric (see [3]).

The problem of existence of Kähler-Einstein metrics on singular Fano varities that have quotient singularities is not well studied even for del Pezzo surfaces with canonical singularities.

Example 1.38. Let $X$ be a cubic surface in $\mathbb{P}^{3}$. Then

- the surface $X$ admits a Kähler-Einstein metric if $\operatorname{Sing}(X)=\varnothing$ (see [180]),

- the surface $X$ does not admit an orbifold Kähler-Einstein metric in the case when it has at least one singular point that is not a singular point of type $\mathbb{A}_{1}$ or $\mathbb{A}_{2}$ (see [51]),

- the surface that is given by the equation

$$
x y z+x y t+x z t+y z t=0 \subset \mathbb{P}^{3} \cong \operatorname{Proj}(\mathbb{C}[x, y, z, t])
$$

admits an orbifold Kähler-Einstein metric and has 4 singular points of type $\mathbb{A}_{1}$ (see [32]),

- the surface that is given by the equation

$$
x y z=t^{3} \subset \mathbb{P}^{3} \cong \operatorname{Proj}(\mathbb{C}[x, y, z, t])
$$

admits an orbifold Kähler-Einstein metric and has 3 singular points of type $\mathbb{A}_{2}$ (see [32]). 
Example 1.39. Let $X$ be a complete intersection of two quadrics in $\mathbb{P}^{4}$. Then

- the surface $X$ admits a Kähler-Einstein metric if $\operatorname{Sing}(X)=\varnothing$ (see [180]),

- the surface $X$ does not admit an orbifold Kähler-Einstein metric in the case when it has at least one singular point that is not an ordinary double point (see [88]),

- if the surface $X \subset \mathbb{P}^{4}$ can be given by the equations

$$
\sum_{i=0}^{4} x_{i}^{2}=\sum_{i=0}^{4} \lambda_{i} x_{i}^{2}=0 \subset \mathbb{P}^{4} \cong \operatorname{Proj}\left(\mathbb{C}\left[x_{0}, \ldots, x_{4}\right]\right)
$$

and $X$ has at most ordinary double points, then $\operatorname{lct}\left(X, \mathbb{Z}_{2}^{4}\right)=1$ (see [116]).

Remark 1.40. Let $X$ be a del Pezzo surface with canonical singularities such that $K_{X}^{2} \leqslant 2$. Then

- the surface $X$ admits a Kähler-Einstein metric in the following cases:

- if $K_{X}^{2}=2$ and $X$ has at most singular points of type $\mathbb{A}_{1}$ or $\mathbb{A}_{2}$ (see [70]);

- if $K_{X}^{2}=1$ and $X$ has at most ordinary double points (see [31]);

- we expect $X$ to admit no Kähler-Einstein metrics if $X$ has relatively bad singularities (cf. [51], [113]).

The numbers $\operatorname{lct}(X)$ and $\operatorname{lct}(X, G)$ play an important role in birational geometry.

Example 1.41. Let $V$ and $\bar{V}$ be varieties with at most terminal and $\mathbb{Q}$-factorial singularities, and let $Z$ be a smooth curve. Suppose that there is a commutative diagram

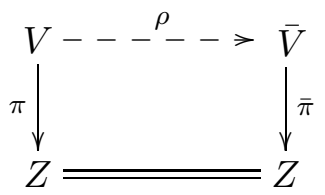

such that $\pi$ and $\bar{\pi}$ are flat morphisms, and $\rho$ is a birational map that induces an isomorphism

$$
V \backslash X \cong \bar{V} \backslash \bar{X}
$$

where $X$ and $\bar{X}$ are scheme fibers of $\pi$ and $\bar{\pi}$ over a point $O \in Z$, respectively. Suppose that

- the fibers $X$ and $\bar{X}$ are irreducible and reduced,

- the divisors $-K_{V}$ and $-K_{\bar{V}}$ are $\pi$-ample and $\bar{\pi}$-ample, respectively,

- the varieties $X$ and $\bar{X}$ have at most log terminal singularities,

and $\rho$ is not an isomorphism. Then it follows from [136] and [32] that

$$
\operatorname{lct}(X)+\operatorname{lct}(\bar{X}) \leqslant 1 \text {, }
$$

where $X$ and $\bar{X}$ are Fano varieties by the adjunction formula.

In general, the inequality 1.42 is sharp (see [152], [72], [73], [137]).

Example 1.43. Let $\pi: V \rightarrow Z$ be a surjective flat morphism such that

- the variety $V$ is a smooth threefold,

- the variety $Z$ is a smooth curve,

- the divisor $-K_{V}$ is $\pi$-ample,

let $X$ be a scheme fiber of the morphism $\pi$ over a point $O \in Z$ such that $X$ is a smooth cubic surface in $\mathbb{P}^{3}$ that has an Eckardt poin $P \in X$ (cf. Definition 4.1), let $L_{1}, L_{2}, L_{3} \subset X$ be the lines that pass through the point $P$. Then it follows from [41] that there is a commutative diagram

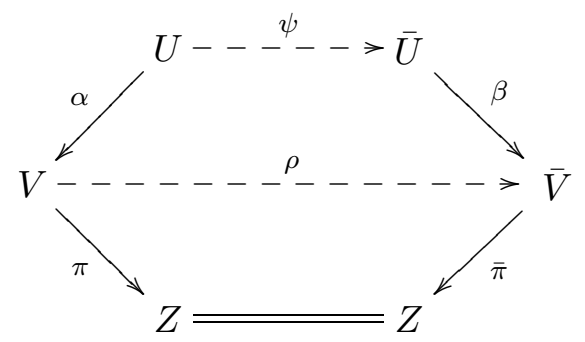

such that $\alpha$ is a blow up of the point $P$, the map $\psi$ is an antiflip in the proper transforms of the curves $L_{1}, L_{2}, L_{3}$, and $\beta$ is a contraction of the proper transform of the fiber $X$. Then 
- the birational map $\rho$ is not an isomorphism,

- the threefold $\bar{V}$ has terminal and $\mathbb{Q}$-factorial singularities,

- the divisor $-K_{\bar{V}}$ is a Cartier $\bar{\pi}$-ample divisor,

- the map $\rho$ induces an isomorphism

$$
V \backslash X \cong \bar{V} \backslash \bar{X}
$$

where $\bar{X}$ is a scheme fiber of $\bar{\pi}$ over the point $O$,

- the surface $\bar{X}$ is a cubic surface with a singular point of type $\mathbb{D}_{4}$.

The latter assertion implies that $\operatorname{lct}(X)+\operatorname{lct}(\bar{X})=1$ (see Examples 1.18 and 1.19).

Global log canonical thresholds can be used to prove that some Fano varieties are non-rational.

Definition 1.44. The variety $X$ is said to be birationally superrigid if the following conditions hold:

- $\operatorname{rk} \operatorname{Pic}(X)=1$;

- the variety $X$ has terminal $\mathbb{Q}$-factorial singularities;

- there is no rational dominant map $\rho: X \rightarrow Y$ such that

- general fiber of the map $\rho$ is rationally connected,

- the inequality $\operatorname{dim}(Y) \geqslant 1$ holds;

- there is no non-biregular birational map $\rho: X \rightarrow Y$ such that

- the variety $Y$ has terminal $\mathbb{Q}$-factorial singularities;

- the equality $\operatorname{rk} \operatorname{Pic}(Y)=1$ holds.

The following result is known as the Noether-Fano inequality (see [40], [96], [22], [159]).

Theorem 1.45. The following conditions are equivalent:

- the variety $X$ is birationally superrigid;

- the following conditions hold:

- the equality $\operatorname{rk} \operatorname{Pic}(X)=1$ holds;

- the variety $X$ has terminal $\mathbb{Q}$-factorial singularities;

- for every linear system $\mathcal{M}$ on the variety $X$ that does not have fixed components, the log pair $(X, \lambda \mathcal{M})$ has canonical singularities, where $K_{X}+\lambda \mathcal{M} \sim_{\mathbb{Q}} 0$.

Proof. Because one part of the required assertion is well-known (see [40], [22], [159]), we prove only another part of the required assertion. Suppose that

- the variety $X$ is birationally superrigid,

- but there is a linear system $\mathcal{M}$ on the variety $X$ such that $\mathcal{M}$ has no fixed components, the singularities of $(X, \lambda \mathcal{M})$ are not canonical, where $K_{X}+\lambda \mathcal{M} \sim \mathbb{Q} 0$.

It follows from [75] that there is birational morphism $\pi: V \rightarrow X$ such that

- the variety $V$ is smooth,

- the proper transform of $\mathcal{M}$ on the variety $V$ has no base points,

and let $\mathcal{B}$ be the proper transform of the linear system $\mathcal{M}$ on the variety $V$. Then

$$
K_{V}+\lambda \mathcal{B} \sim_{\mathbb{Q}} \pi^{*}\left(K_{X}+\lambda \mathcal{M}\right)+\sum_{i=1}^{r} a_{i} E_{i} \sim_{\mathbb{Q}} \sum_{i=1}^{r} a_{i} E_{i},
$$

where $E_{i}$ is an exceptional divisor of $\pi$, and $a_{i} \in \mathbb{Q}$.

It follows from [9] that there is a commutative diagram

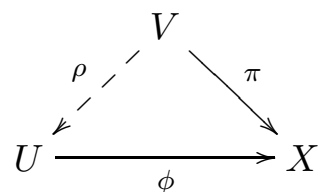

such that $\rho$ is a birational map, the morphism $\phi$ is birational, the divisor

$$
K_{U}+\lambda \rho(\mathcal{B}) \sim_{\mathbb{Q}} \phi^{*}\left(K_{X}+\lambda \mathcal{M}\right)+\sum_{i=1}^{r} a_{i} \rho\left(E_{i}\right) \sim_{\mathbb{Q}} \sum_{i=1}^{r} a_{i} \rho\left(E_{i}\right)
$$


is $\phi$-nef, the variety $U$ is $\mathbb{Q}$-factorial, the $\log$ pair $(U, \lambda \rho(\mathcal{B})$ has terminal singularities.

The morphism $\phi$ is not an isomorphism. It follows from [169, 1.1] that

$$
a_{i}>0 \Longrightarrow \operatorname{dim}\left(\rho\left(E_{i}\right)\right) \leqslant \operatorname{dim}(X)-2,
$$

but it follows from the construction of the map $\rho$ that there is $k \in\{1, \ldots, r\}$ such that

- the inequality $a_{k}<0$ holds,

- the subvariety $\rho\left(E_{k}\right) \subset U$ is a divisor,

because the singularities of the log pair $(X, \lambda \mathcal{M})$ are not canonical.

The divisor $K_{U}+\lambda \rho(\mathcal{B})$ is not pseudo-effective. Then it follows from [9] that there is a diagram

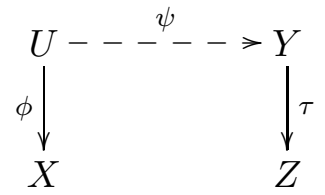

such that $\psi$ is a birational map, the morphism $\tau$ is a Mori fibred space (see [102]), and the divisor

$$
-\left(K_{Y}+\lambda(\psi \circ \rho)(\mathcal{B})\right)
$$

is $\tau$-ample. The variety $Y$ has terminal $\mathbb{Q}$-factorial singularities, and $\operatorname{rk} \operatorname{Pic}(Y / Z)=1$. Then

- the birational map $\psi \circ \rho \circ \pi^{-1}$ is not an isomorphism, because $K_{X}+\lambda \mathcal{M} \sim_{\mathbb{Q}} 0$,

- general fiber of the morphism $\tau$ is rationally connected (see [193]),

which contradicts the assumption that $X$ is birationally superrigid.

Birationally superrigid Fano varieties are non-rational. In particular, if the variety $X$ is birationally superrigid, then $\operatorname{dim}(X) \neq 2$ (cf. [117], [118], [95]).

Example 1.46. It follows from [97] that smooth quartic hypersurface

$$
x^{4}+x w^{3}+y^{4}-6 y^{2} z^{2}+z^{4}+t^{4}+t^{3} w=0 \subset \mathbb{P}^{4} \cong \operatorname{Proj}(\mathbb{C}[x, y, z, t, w])
$$

is smooth and unirational (cf. [120]) and birationally superrigid (cf. [38]).

Example 1.47. The following smooth Fano varieties are birationally superrigid:

- a smooth hypersurface in $\mathbb{P}^{n}$ of degree $n$ for $4 \leqslant n \leqslant 12$ (see [97, [147], [19], [155], [59]);

- a general hypersurface in $\mathbb{P}^{n}$ of degree $n \geqslant 4$ (see [151]);

- a smooth hypersurface in $\mathbb{P}\left(1^{n+1}, n\right)$ of degree $2 n \geqslant 6$ (see [94, [148]).

- a general complete intersection of hypersurfaces $F_{1}, \ldots, F_{k} \subset \mathbb{P}^{n}$ such that

$$
\sum_{i=1}^{k} \operatorname{deg}\left(F_{i}\right)=n \geqslant 3 k+1 \geqslant 7,
$$

where $\operatorname{deg}\left(F_{k}\right) \geqslant \ldots \geqslant \operatorname{deg}\left(F_{2}\right) \geqslant \operatorname{deg}\left(F_{1}\right) \geqslant 2$ (see [154);

- a smooth fourfold complete intersection in $\mathbb{P}^{6}$ of degree 8 containing no planes (see [21]);

- a smooth Fano variety $X$ such that there is a double cover

$$
\tau: X \longrightarrow V \subset \mathbb{P}^{n}
$$

where $V$ is a hypersurface, $\tau$ is ramified in a divisor $R \in\left|\mathcal{O}_{\mathbb{P}^{n}}(2 n-2 \operatorname{deg}(V))\right|_{V} \mid$, and

- either $\operatorname{deg}(V)=2$ and $n \geqslant 5$ (see [148]),

- or $V$ and $R \in\left|\mathcal{O}_{\mathbb{P}^{n}}(2 n-2 \operatorname{deg}(V))\right|_{V} \mid$ are general and $n \geqslant 5$ (see [153]),

- or $3 \leqslant \operatorname{deg}(V) \leqslant 4$ and $n \geqslant 8$ (see [24]).

- a sextic hypersurface in $\mathbb{P}^{6}$ with at most ordinary double points (see [25]).

Example 1.48. Let $\pi: X \rightarrow \mathbb{P}^{3}$ be a double cover branched along a surface $S \subset \mathbb{P}^{3}$ of degree 6 such that the sextic surface $S$ has at most ordinary double points. Then

- the inequality $|\operatorname{Sing}(S)| \leqslant 65$ holds (see [84], [188]),

- for any $65 \geqslant k \in \mathbb{Z}_{>0}$, there exists $S \subset \mathbb{P}^{3}$ such that $|\operatorname{Sing}(S)|=k$ (see [18], [6]),

- the variety $X$ is birationally superrigid in the case when $\operatorname{rk} \operatorname{Cl}(X)=1$ (see [97], 34]),

- the equality $\operatorname{rk} \operatorname{Cl}(X)=1$ holds if $|\operatorname{Sing}(S)| \leqslant 14$ (see [34]), 
- suppose that the surface $S$ is a Barth sextic (see [6]) that is given by

$4\left(\tau^{2} x^{2}-y^{2}\right)\left(\tau^{2} y^{2}-z^{2}\right)\left(\tau^{2} z^{2}-x^{2}\right)=t^{2}(1+2 \tau)\left(x^{2}+y^{2}+z^{2}-t^{2}\right)^{2} \subset \mathbb{P}^{3} \cong \operatorname{Proj}(\mathbb{C}[x, y, z, t])$,

where $\tau=(1+\sqrt{5}) / 2$; then $\operatorname{rk} \operatorname{Cl}(X)=14$ (see [56]) and the diagram

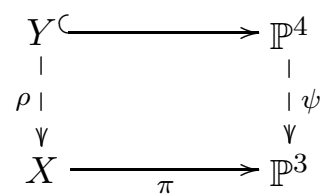

commutes (see [56], [140]), where

- the variety $Y$ is a determinantal quartic threefold in $\mathbb{P}^{4}$ such that $|\operatorname{Sing}(Y)|=42$,

- the map $\psi$ is the projection from a singular point of the quartic $Y$,

- the map $\rho$ is a birational map,

which implies that the threefold $X$ is rational.

The following result is proved in [157].

Theorem 1.49. Let $X_{1}, X_{2}, \ldots, X_{r}$ be birationally superrigid Fano varieties such that $\operatorname{lct}\left(X_{1}\right) \geqslant$ $1, \operatorname{lct}\left(X_{2}\right) \geqslant 1, \ldots, \operatorname{lct}\left(X_{r}\right) \geqslant 1$. Then

- the variety $X_{1} \times \ldots \times X_{r}$ is non-rational and

$$
\operatorname{Bir}\left(X_{1} \times \ldots \times X_{r}\right)=\operatorname{Aut}\left(X_{1} \times \ldots \times X_{r}\right),
$$

- for every rational dominant map

$$
\rho: X_{1} \times \ldots \times X_{r} \rightarrow Y
$$

whose general fiber is rationally connected, there is a commutative diagram

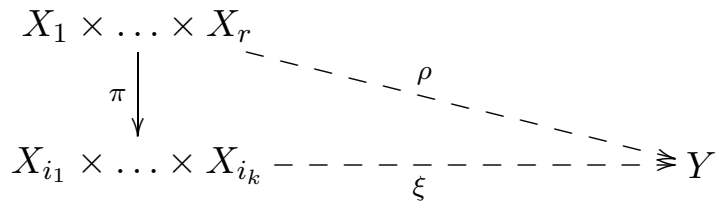

for some $\left\{i_{1}, \ldots, i_{k}\right\} \subseteq\{1, \ldots, r\}$, where $\xi$ is a birational map, and $\pi$ is a projection.

Varieties satisfying all hypotheses of Theorem 1.49 exist (see Examples 1.11, 1.13, 1.14, 1.47).

Example 1.50. Let $X$ be a hypersurface that is given by

$$
w^{2}=x^{6}+y^{6}+z^{6}+t^{6}+x^{2} y^{2} z t \subset \mathbb{P}(1,1,1,1,3) \cong \operatorname{Proj}(\mathbb{C}[x, y, z, t, w]),
$$

where $\operatorname{wt}(x)=\operatorname{wt}(y)=\operatorname{wt}(z)=\operatorname{wt}(t)=1$ and $\operatorname{wt}(w)=3$. Then

- the threefold $X$ is smooth and birationally superrigid (see [94]),

- it follows from [37] that the equality $\operatorname{lct}(X)=1$ holds.

Example 1.51. Let $X$ be a hypersurface that is given by

$$
w^{2}=\sum_{i=0}^{n} x_{i}^{2 n}+\varepsilon \sum_{i=1}^{n-4}\left(\sum_{j=0}^{n} a_{i j} x_{i}\right)^{2 n} \subset \mathbb{P}\left(1^{n+1}, n\right) \cong \operatorname{Proj}\left(\mathbb{C}\left[x_{0}, x_{1}, \ldots, x_{n}, w\right]\right)
$$

for some $\varepsilon \in \mathbb{C} \ni a_{i j}$, where $\operatorname{wt}\left(x_{i}\right)=1$ and $\operatorname{wt}(w)=n$. Suppose that any $n+1$ forms among

$$
x_{0}, x_{1}, \ldots, x_{n}, \sum_{i=0}^{n} a_{1 i} x_{i}, \sum_{i=0}^{n} a_{2 i} x_{i}, \ldots, \sum_{i=0}^{n} a_{n-4 i} x_{i}
$$

are linearly independent. Put $\Delta=\max \left\{\left|a_{i j}\right|\right\}$. Suppose that the inequalities $n \geqslant 8$ and

$$
1>|\varepsilon|(2 n \Delta+2 \Delta)^{2 m}
$$


hold. Then $X$ is birationally superrigid and $\operatorname{lct}(X)=1$ (see [148], [160]). The hypersurface

$$
w^{2}=n^{2 n^{2}} \sum_{i=0}^{n} x_{i}^{2 n}+\sum_{i=1}^{n-4}\left(\sum_{j=0}^{n}(j+1)^{i} x_{i}\right)^{2 n}
$$

is birationally superrigid, and its global log canonical threshold is 1 (see [160]).

Suppose, in addition, that the subgroup $G \subset \operatorname{Aut}(X)$ is finite (cf. [71]).

Definition 1.52. The Fano variety $X$ is $G$-birationally superrigid if

- the $G$-invariant subgroup of the group $\mathrm{Cl}(X)$ is isomorphic to $\mathbb{Z}$,

- the variety $X$ has terminal singularities,

- there is no $G$-equivariant rational dominant map $\rho: X \rightarrow Y$ such that

- general fiber of the map $\rho$ is rationally connected,

- the inequality $\operatorname{dim}(Y) \geqslant 1$ holds,

- there is no $G$-equivariant non-biregular birational map $\rho: X \rightarrow Y$ such that

- the $G$-invariant subgroup of the group $\mathrm{Cl}(Y)$ is isomorphic to $\mathbb{Z}$,

- the variety $Y$ has terminal singularities.

Arguing as in the proof of Theorem 1.45, we obtain the following result.

Theorem 1.53. The following conditions are equivalent:

- the variety $X$ is $G$-birationally superrigid;

- the following conditions hold:

- the $G$-invariant subgroup of the group $\mathrm{Cl}(X)$ is isomorphic to $\mathbb{Z}$;

- the variety $X$ has terminal singularities;

- for every $G$-invariant linear system $\mathcal{M}$ on variety $X$ that has no fixed components, the log pair $(X, \lambda \mathcal{M})$ is canonical, where $K_{X}+\lambda \mathcal{M} \sim_{\mathbb{Q}} 0$.

If $X$ is birationally superrigid, then $X$ is $G$-birationally superrigid for any $G \subset \operatorname{Aut}(X)$.

Example 1.54. Let $X$ be a smooth surface in $\mathbb{P}(1,1,2,3)$ of degree 6 such that the $G$-invariant subgroup of the group $\operatorname{Pic}(X)$ is $\mathbb{Z}$. Then $X$ is $G$-birationally superrigid (see [117, [118, [95]).

The proof of Theorem 1.49 implies the following result (see [31]).

Theorem 1.55. Let $X_{i}$ be a Fano variety, and let $G_{i} \subset \operatorname{Aut}\left(X_{i}\right)$ be a finite subgroup such that

- the variety $X_{i}$ is $G_{i}$-birationally superrigid,

- the inequality $\operatorname{lct}\left(X_{i}, G_{i}\right) \geqslant 1$ holds for any $i=1, \ldots, r$.

Then the following assertions hold:

- there is no $G_{1} \times \ldots \times G_{r}$-equivariant birational map $\rho: X_{1} \times \ldots \times X_{r} \rightarrow \mathbb{P}^{n}$;

- every $G_{1} \times \ldots \times G_{r}$-equivariant birational automorphism of $X_{1} \times \ldots \times X_{r}$ is biregular;

- for every $G_{1} \times \ldots \times G_{r}$-equivariant rational dominant map

$$
\rho: X_{1} \times \ldots \times X_{r} \rightarrow Y
$$

whose general fiber is rationally connected, there a commutative diagram

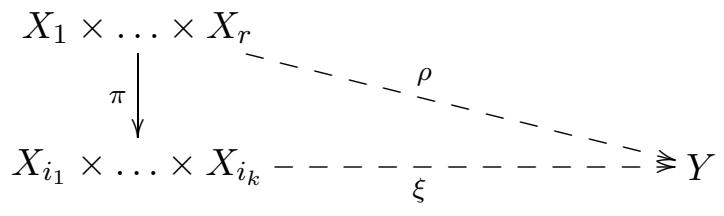

where $\xi$ is a birational map, $\pi$ is a natural projection, and $\left\{i_{1}, \ldots, i_{k}\right\} \subseteq\{1, \ldots, r\}$.

Varieties satisfying all hypotheses of Theorem 1.55 do exist (see Example 1.25).

Example 1.56. The simple group $A_{6}$ is a group of automorphisms of the sextic

$$
10 x^{3} y^{3}+9 z x^{5}+9 z y^{5}+27 z^{6}=45 x^{2} y^{2} z^{2}+135 x y z^{4} \subset \mathbb{P}^{2} \cong \operatorname{Proj}(\mathbb{C}[x, y, z]),
$$


which induces an embedding $\mathrm{A}_{6} \subset \operatorname{Aut}\left(\mathbb{P}^{2}\right)$. It follows from 44 that $\mathbb{P}^{2}$ is $\mathrm{A}_{6}$-birationally superrigid. But the equality $\operatorname{lct}\left(\mathbb{P}^{2}, \mathrm{~A}_{6}\right)=2$ holds (see [31]). Thus, there is an induced embedding

$$
\mathrm{A}_{6} \times \mathrm{A}_{6} \cong \Omega \subset \operatorname{Bir}\left(\mathbb{P}^{4}\right)
$$

such that $\Omega$ is not conjugate to any subgroup in $\operatorname{Aut}\left(\mathbb{P}^{4}\right)$ by Theorem 1.55 .

Example 1.57. Suppose that $X$ be a smooth cubic surface in $\mathbb{P}^{3}$ that is given by

$$
x^{2} y+x z^{2}+z t^{2}+t x^{2}=0 \subset \mathbb{P}^{3} \cong \operatorname{Proj}(\mathbb{C}[x, y, z, t]) .
$$

Then $\operatorname{Aut}(X) \cong \mathrm{S}_{5}($ see [52]). Hence, by [31]

$$
\operatorname{lct}\left(X, \mathrm{~S}_{5}\right)=\operatorname{lct}\left(X, \mathrm{~A}_{5}\right)=2 \text {, }
$$

and the surface $X$ is $A_{5}$-birationally superrigid (see Example 1.54).

Let us consider Fano varieties that are close to being birationally superrigid.

Definition 1.58. The Fano variety $X$ is birationally rigid 3

- the equality $\operatorname{rk} \operatorname{Pic}(X)=1$ holds,

- the variety $X$ has $\mathbb{Q}$-factorial and terminal singularities,

- there is no rational dominant map $\rho: X \rightarrow Y$ such that

- a general fiber of the map $\rho$ is rationally connected,

- the inequality $\operatorname{dim}(Y) \geqslant 1$ holds,

- there is no birational map $\rho: X \rightarrow Y$ such that

- the varieties $Y$ and $X$ are not biregular,

- the variety $Y$ has terminal $\mathbb{Q}$-factorial singularities,

- the equality $\operatorname{rk} \operatorname{Pic}(Y)=1$ holds.

Arguing as in the proof of Theorem 1.45, we obtain the following result.

Theorem 1.59. The following conditions are equivalent:

- the variety $X$ is birationally rigid;

- the following conditions hold:

- the equality $\operatorname{rk} \operatorname{Pic}(X)=1$ holds;

- the variety $X$ has $\mathbb{Q}$-factorial and terminal singularities;

- for every linear system $\mathcal{M}$ on the Fano variety $X$ that does not have fixed components, there is birational automorphism $\xi \in \operatorname{Bir}(X)$ such that the log pair

$$
(X, \lambda \xi(\mathcal{M}))
$$

has canonical singularities, where $K_{X}+\lambda \xi(\mathcal{M}) \sim_{\mathbb{Q}} 0$.

Remark 1.60. For every $n \geqslant 5$, there exists a smooth Fano variety $X$ of dimension $n$ such that

$$
\operatorname{Pic}(X)=\mathbb{Z}\left[-K_{X}\right]
$$

and the variety $X$ is not birationally rigid (see [17]).

Birationally rigid Fano varieties are non-rational (see [40], [96], 222], [159]).

Example 1.61. The following varieties are birationally rigid but not birationally superrigid:

- a general complete intersection of a quadric and a cubic in $\mathbb{P}^{5}$ (see [99]);

- a smooth double cover of a quadric in $\mathbb{P}^{4}$ branched over a surface of degree 8 (see [94]).

One usually seeks for the birational automorphism in Definition 1.58 in a given set of birational automorphisms. This leads to the following definition.

\footnotetext{
${ }^{3}$ There are several definitions of birational rigidity and birational superrigidity (see [40, 442, 96], 22], 159]).
} 
Definition 1.62. Suppose that $X$ is birationally rigid. A subset $\Gamma \subset \operatorname{Bir}(X)$ untwists all maximal singularities if for every linear system $\mathcal{M}$ on the variety $X$ that has no fixed components, there is a birational automorphism $\xi \in \Gamma$ such that the $\log$ pair

$$
(X, \lambda \xi(\mathcal{M}))
$$

has canonical singularities, where $\lambda$ is a rational number such that $K_{X}+\lambda \xi(\mathcal{M}) \sim_{\mathbb{Q}} 0$.

If $X$ is birationally rigid and there is $\Gamma \subset \operatorname{Bir}(X)$ that untwists all maximal singularities, then the group $\operatorname{Bir}(X)$ is generated by $\Gamma$ and $\operatorname{Aut}(X)$.

Example 1.63. Let $X$ be a sufficiently general hypersurface in $\mathbb{P}^{n}$ of degree $n \geqslant 5$ that has one ordinary singular point $O=\operatorname{Sing}(X)$ of multiplicity $n-2$. Then the projection

$$
\psi: X \rightarrow \mathbb{P}^{n-1}
$$

from the point $O$ induces an involution that untwists all maximal singularities (see [156]).

If $X$ is defined over a perfect field, then Definition [1.58 still makes sense (see [117], [118], [95]).

Definition 1.64. The variety $X$ is universally birationally rigid if for any variety $U$, the variety

$$
X \otimes \operatorname{Spec}(\mathbb{C}(U))
$$

is birationally rigid over a field of rational functions $\mathbb{C}(U)$ of the variety $U$.

Example 1.65. Let $X$ be a smooth Fano threefold such that there is a double cover

$$
\pi: X \longrightarrow Q \subset \mathbb{P}^{3},
$$

where $Q$ is a quadric threefold, and $\pi$ is branched in a surface $S \subset Q$ of degree 8 . Put

$$
\mathcal{C}=\left\{C \subset X \mid C \text { is a smooth curve such that }-K_{X} \cdot C=1\right\},
$$

then $\mathcal{C}$ is a one-dimensional family. For every curve $C \in \mathcal{C}$ there is a commutative diagram

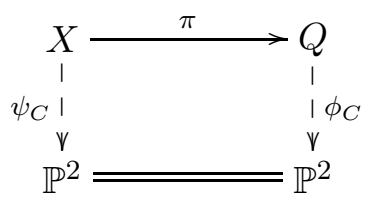

where $\phi_{C}$ is a projection from the line $\pi(C)$. General fiber of the map $\psi_{C}$ is an elliptic curve, the map $\psi_{C}$ induces an elliptic fibration with a section and an involution $\tau_{C} \in \operatorname{Bir}(X)$. Then

$$
\psi_{C} \in \operatorname{Aut}(X) \Longleftrightarrow C \subset S,
$$

and $S$ contains no curves in $\mathcal{C}$ if $X$ is general. It follows from [94] that there is an exact sequence

$$
1 \longrightarrow \Gamma \longrightarrow \operatorname{Bir}(X) \longrightarrow \operatorname{Aut}(X) \longrightarrow 1
$$

where $\Gamma$ is a free product of subgroups that are generated by non-biregular birational involutions constructed above. Hence the Fano variety $X$ is universally birationally rigid (see [94]).

Example 1.66. Let $X$ be a quartic threefold in $\mathbb{P}^{4}$ that has at most ordinary double points. Then

- the inequality $|\operatorname{Sing}(X)| \leqslant 45$ holds (see [186]),

- in general, the variety $X$ is not birationally superrigid if $\operatorname{Sing}(X) \neq \varnothing$,

- the variety $X$ is universally birationally rigid if $\operatorname{rk} \mathrm{Cl}(X)=1$ (see [97, [149], [125], [172]),

- the inequality $\mathrm{rk} \mathrm{Cl}(X) \leqslant 16$ holds (see [100, [101]),

- the equality $\operatorname{rk} \operatorname{Cl}(X)=1$ holds if $|\operatorname{Sing}(X)| \leqslant 8$ (see [23]),

- the equality $\mathrm{rk} \mathrm{Cl}(X)=1$ holds if the following conditions hold:

- the inequality $|\operatorname{Sing}(X)| \leqslant 12$ holds;

- the quartic $X$ contains neither planes or quadric surfaces (see [171]);

- in the case when $|\operatorname{Sing}(X)|=45$, it follows from 83 that $X$ can be given by the equation

$$
w^{4}-w\left(x^{3}+y^{3}+z^{3}+t^{3}\right)+3 x y z t=0 \subset \mathbb{P}^{4} \cong \operatorname{Proj}(\mathbb{C}[x, y, z, t, w]),
$$

the quartic $X$ is determinantal and rational, and $\operatorname{rkCl}(X)=16$ (see [76], [100], [101]). 
Birationally superrigid Fano varieties are universally birationally rigid.

Definition 1.67. Suppose that $X$ is universally birationally rigid. A subset $\Gamma \subset \operatorname{Bir}(X)$ universally untwists all maximal singularities if for every variety $U$ the induced subset

$$
\Gamma \subset \operatorname{Bir}(X) \subseteq \operatorname{Bir}(X \otimes \operatorname{Spec}(\mathbb{C}(U)))
$$

untwists all maximal singularities on $X \otimes \operatorname{Spec}(\mathbb{C}(U))$.

An identity map universally untwists all maximal singularities if $X$ is birationally superrigid.

Remark 1.68. Suppose that $X$ is birationally rigid, and $\operatorname{dim}(X) \neq 1$. Let $\Gamma \subseteq \operatorname{Bir}(X)$ be a subset. It follows from [107] that the following conditions are equivalent:

- the subset $\Gamma$ universally untwists all maximal singularities;

- the subset $\Gamma$ untwists all maximal singularities, and $\operatorname{Bir}(X)$ is countable.

Example 1.69. In the assumptions of Example 1.15, suppose that $X$ is general. Then

- the hypersurface $X$ is universally birationally rigid (see [43]),

- there are involutions $\tau_{1}, \ldots, \tau_{k} \in \operatorname{Bir}(X)$ such that that the sequence of groups

$$
1 \longrightarrow\left\langle\tau_{1}, \ldots, \tau_{k}\right\rangle \longrightarrow \operatorname{Bir}(X) \longrightarrow \operatorname{Aut}(X) \longrightarrow 1
$$

is exact (see [43], 35]), where $\left\langle\tau_{1}, \ldots, \tau_{k}\right\rangle$ is a subgroup generated by $\tau_{1}, \ldots, \tau_{k}$,

- the subgroup $\left\langle\tau_{1}, \ldots, \tau_{k}\right\rangle$ universally untwists all maximal singularities (see [43]).

All relations between the involutions $\tau_{1}, \ldots, \tau_{k}$ are found in [35]. The papers [164], [165], 35], [26] classify all maps $\psi: X \rightarrow \mathbb{P}^{2}$ such that the diagram

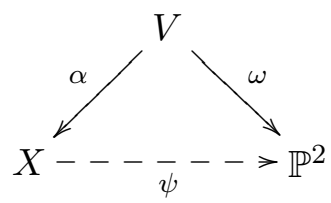

commutes, where $\alpha$ is a birational morphism, and $\omega$ is an elliptic fibration. The papers [164, [165], 36] classify all maps $\phi: X \rightarrow \mathbb{P}^{1}$ such that the diagram

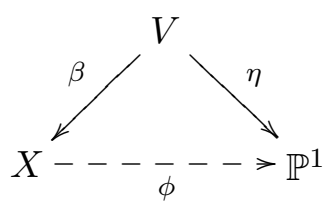

commutes, where $\beta$ is a birational morphism, and $\eta$ is a fibration into surfaces of Kodaira dimension zero.

Let $X_{1}, \ldots, X_{r}$ be Fano varieties that have at most $\mathbb{Q}$-factorial and terminal singularities, let $\pi_{i}: X_{1} \times \ldots \times X_{i-1} \times X_{i} \times X_{i+1} \times \ldots \times X_{r} \longrightarrow X_{1} \times \ldots \times X_{i-1} \times \widehat{X}_{i} \times X_{i+1} \times \ldots \times X_{r}$

be a natural projection, and let $\mathcal{X}_{i}$ be a scheme general fiber of the projection $\pi_{i}$, which is defined over $\mathbb{C}\left(X_{1} \times \ldots \times X_{i-1} \times \widehat{X}_{i} \times X_{i+1} \times \ldots \times X_{r}\right)$. Suppose that $\operatorname{rk} \operatorname{Pic}\left(X_{1}\right)=\ldots=\operatorname{rk} \operatorname{Pic}\left(X_{r}\right)=1$.

Remark 1.70. There are natural embeddings of groups

$$
\prod_{i=1}^{r} \operatorname{Bir}\left(X_{i}\right) \subseteq\left\langle\operatorname{Bir}\left(\mathcal{X}_{1}\right), \ldots, \operatorname{Bir}\left(\mathcal{X}_{r}\right)\right\rangle \subseteq \operatorname{Bir}\left(X_{1} \times \ldots \times X_{r}\right) .
$$

The following generalization of Theorem 1.49 holds (see [27]).

Theorem 1.71. Suppose that $X_{1}, X_{2}, \ldots, X_{r}$ are universally birationally rigid. Then

- the variety $X_{1} \times \ldots \times X_{r}$ is non-rational and

$$
\operatorname{Bir}\left(X_{1} \times \ldots \times X_{r}\right)=\left\langle\operatorname{Bir}\left(\mathcal{X}_{1}\right), \ldots, \operatorname{Bir}\left(\mathcal{X}_{r}\right), \operatorname{Aut}\left(X_{1} \times \ldots \times X_{r}\right)\right\rangle,
$$


- for every rational dominant map $\rho: X_{1} \times \ldots \times X_{r} \rightarrow Y$, whose general fiber is rationally connected, there is a subset $\left\{i_{1}, \ldots, i_{k}\right\} \subseteq\{1, \ldots, r\}$ and a commutative diagram

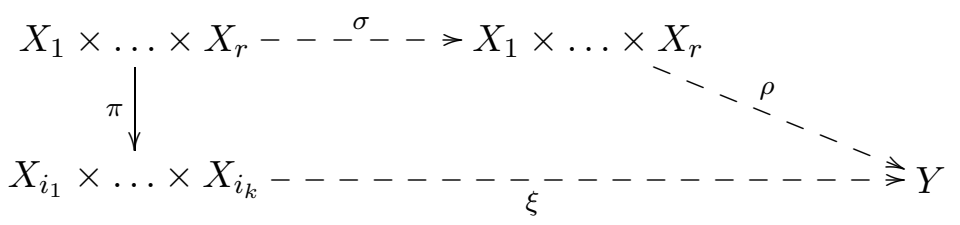

where $\xi$ and $\sigma$ are birational maps, and $\pi$ is a projection,

in the case when the inequalities $\operatorname{lct}\left(X_{1}\right) \geqslant 1, \operatorname{lct}\left(X_{2}\right) \geqslant 1, \ldots, \operatorname{lct}\left(X_{r}\right) \geqslant 1$ hold.

Corollary 1.72. Suppose that there are subgroups $\Gamma_{1} \subseteq \operatorname{Bir}\left(X_{1}\right), \ldots, \Gamma_{r} \subseteq \operatorname{Bir}\left(X_{r}\right)$ that universally untwists all maximal singularities, and $\operatorname{lct}\left(X_{1}\right) \geqslant 1, \operatorname{lct}\left(X_{2}\right) \geqslant 1, \ldots, \operatorname{lct}\left(X_{r}\right) \geqslant 1$. Then

$$
\operatorname{Bir}\left(X_{1} \times \ldots \times X_{r}\right)=\left\langle\prod_{i=1}^{r} \Gamma_{i}, \operatorname{Aut}\left(X_{1} \times \ldots \times X_{r}\right)\right\rangle .
$$

The following four examples are implied by Examples 1.14, 1.15, 1.47, 1.69 and [123].

Example 1.73. Let $X$ be a general hypersurface in $\mathbb{P}(1,1,4,5,10)$ of degree 20 . The sequence

$$
1 \longrightarrow \prod_{i=1}^{m}\left(\mathbb{Z}_{2} * \mathbb{Z}_{2}\right) \longrightarrow \operatorname{Bir}(\underbrace{X \times \ldots \times X}_{m \text { times }}) \longrightarrow \mathrm{S}_{m} \longrightarrow 1
$$

is exact, where $\mathrm{S}_{m}$ is a permutation group, and $\mathbb{Z}_{2} * \mathbb{Z}_{2}$ is the infinite dihedral group.

Example 1.74. Let $X$ be a general hypersurface in $\mathbb{P}(1,1,3,4,5)$ of degree 13 . Then

$$
\operatorname{Bir}(X \times V) \cong \mathbb{Z}_{2} * \mathbb{Z}_{2} * \mathbb{Z}_{2}
$$

where $V$ is a general hypersurface in $\mathbb{P}^{n}$ of degree $n \geqslant 6$.

Example 1.75. Let $X$ be a general hypersurface in $\mathbb{P}(1,1,2,3,3)$ of degree 9 . Then

$$
\operatorname{Bir}(X \times V) \cong\left\langle a, b, c \mid a^{2}=b^{2}=c^{2}=(a b c)^{2}=1\right\rangle,
$$

where $V$ is a general hypersurface in $\mathbb{P}^{n}$ of degree $n \geqslant 6$.

Example 1.76. Let $X$ be a general hypersurface in $\mathbb{P}(1,1,2,2,3)$ of degree 8 . Then

$$
\operatorname{Bir}(X \times V) \cong \mathbb{Z}_{2} * \mathbb{Z}_{2} * \mathbb{Z}_{2} * \mathbb{Z}_{2} * \mathbb{Z}_{2}
$$

where $V$ is a general hypersurface in $\mathbb{P}^{n}$ of degree $n \geqslant 6$.

Suppose now that $X$ is a smooth Fano threefold (see [98]). Let

$$
I(X) \in\{1.1,1.2, \ldots, 1.17,2.1, \ldots, 2.36,3.1, \ldots, 3.31,4.1, \ldots, 4.13,5.1, \ldots, 5.7,5.8\}
$$

be the ordinal number of the deformation type of the threefold $X$ in the notation of Table 1 . Remark 1.77. The threefold $X$ lies in 105 deformation families (see [92, 93], [126], 128], [129], [127]).

The main purpose of this paper is to prove the following result.

Theorem 1.78. The following assertions hold:

- $\operatorname{lct}(X)=1 / 5$ for $\Xi(X) \in\{2.36,3.29\}$;

- $\operatorname{lct}(X)=1 / 4$ for

$$
J(X) \in\{1.17,2.28,2.30,2.33,2.35,3.23,3.26,3.30,4.12\} ;
$$

- $\operatorname{lct}(X)=1 / 3$ for

$$
J(X) \in\{1.16,2.29,2.31,2.34,3.9,3.18, \ldots, 3.22,3.24,3.25,3.28,3.31,4.4,4.8, \ldots 4.11,5.1,5.2\} ;
$$

- $\operatorname{lct}(X)=3 / 7$ for $I(X)=4.5$; 
- $\operatorname{lct}(X)=1 / 2$ for

$J(X) \in\left\{\begin{array}{l}1.11,1.12,1.13,1.14,1.15,2.1,2.3,2.18,2.25,2.27,2.32,3.4,3.10,3.11,3.12, \\ 3.14,3.15,3,16,3.17,3.24,3.27,4.1,4.2,4.3,4.6,4.7,5.3,5.4,5.5,5.6,5.7,5.8\end{array}\right\} ;$

- if the threefold $X$ is general in moduli, then

$$
\operatorname{lct}(X)=\left\{\begin{array}{l}
1 / 3 \text { if } \exists(X)=2.23, \\
1 / 2 \text { if } \exists(X) \in\{2.5,2.8,2.10,2.11,2.14,2.15,2.19,2.24,2.26,3.2,3.5, \ldots, 3.8,4.13\}, \\
2 / 3 \text { if } \exists(X)=3.3, \\
3 / 4 \text { if } \Xi(X) \in\{2.4,3.1\}, \\
1 \text { if } \Xi(X)=1.1 .
\end{array}\right.
$$

Hence, if the threefold $X$ is general in moduli, then we do not know $\operatorname{lct}(X)$ only when

$I(X) \in\{1.2,1.3,1.4,1.5, \ldots, 1.10,2.2,2.6,2.7,2.9,2.12,2.13,2.16,2.17,2.20,2.21,2.22,3.13\}$,

and the generality condition in Theorem 1.78 can not be omitted in many cases.

Example 1.79. Suppose that $I(X)=4.13$. Note that this deformation type was omitted in [126], and it has been discovered only twenty years later (see [127]). There is a birational morphism

$$
\alpha: X \longrightarrow \mathbb{P}^{1} \times \mathbb{P}^{1} \times \mathbb{P}^{1}
$$

that contracts a surface $E \subset X$ to a curve $C$ such that $C \cdot F_{1}=C \cdot F_{2}=1$ and $C \cdot F_{3}=3$, where

$$
F_{1} \cong F_{2} \cong F_{3} \cong \mathbb{P}^{1} \times \mathbb{P}^{1}
$$

are fibers of three different projections $\mathbb{P}^{1} \times \mathbb{P}^{1} \times \mathbb{P}^{1} \rightarrow \mathbb{P}^{1}$, respectively. Then

$$
\operatorname{lct}(X)=1 / 2
$$

by Theorem 1.78 if $X$ is general. There is a surface $G \in\left|F_{1}+F_{2}\right|$ such that $C \subset G$. Then

$$
-K_{X} \sim 2 \bar{G}+E+\bar{F}_{3},
$$

where $\bar{F}_{3} \subset X \supset \bar{G}$ are proper transforms of $F_{3}$ and $G$, respectively. Then $\operatorname{lct}(X) \leqslant 1 / 2$. But

$$
\operatorname{lct}(X) \leqslant \operatorname{lct}\left(X, 2 \bar{G}+E+\bar{F}_{3}\right) \leqslant 4 / 9<1 / 2
$$

in the case when the intersection $F_{3} \cap C$ consists of a single point.

We hope that the proof of Theorem 1.78 can be used to

- study the slope stability of the threefold $X$ in the sense of [162] and [163,

- study the problem of existence of a Kähler-Einstein metric on the threefold $X$,

- compute $\operatorname{lct}(X, G)$ for various subgroups $G \subset \operatorname{Aut}(X)$.

Remark 1.80. The stability of the tangent bundle of $X$ was studied in [176]. It is known that

- the tangent bundle of $X$ is unstable with respect to $-K_{X}$ when

$$
I(X) \in\{2.35,2.36,3.29,3.30,3.31,4.11,4.12\},
$$

- the tangent bundle of $X$ is semistable with respect to $-K_{X}$ when

$$
I(X) \in\{2.33,2.34,3.27,3.28,4.10,5.2, \ldots, 5.8\},
$$

- the tangent bundle of $X$ is stable with respect to $-K_{X}$ when

$$
J(X) \notin\{2.33,2.34,2.35,2.36,3.27, \ldots, 3.31,4.10,4.11,4.12,5.2, \ldots, 5.8\} .
$$

We organize the paper in the following way:

- in Section 2, we consider auxiliary results that are used in the proof of Theorem 1.78,

- in Section 3, we find the global log canonical threshold of the Mukai-Umemura threefold;

- in Section 4, we prove Theorem 4.2 that is required for Example 5.4 and Lemma 8.2;

- in Section 5, we consider facts on surfaces that are used in the proof of Theorem 1.78,

- in Section 6, we compute global log canonical thresholds of toric Fano varieties; 
- in Section 7, we prove Theorem 1.78 for smooth Fano threefolds of index 2, i.e., for

$$
I(X) \in\{1.11,1.12,1.13,1.14,1.15,2.32,2.35,3.27\} ;
$$

- in Section 8, we prove Theorem 1.78 in the case when $\operatorname{rk} \operatorname{Pic}(X)=2$;

- in Section 9, we prove Theorem 1.78 in the case when $\operatorname{rk} \operatorname{Pic}(X)=3$;

- in Section 10, we prove Theorem 1.78 in the case when $\operatorname{rk} \operatorname{Pic}(X) \geqslant 4$;

- in Section 11, we find upper bounds for $\operatorname{lct}(X)$ in the case when

$$
I(X) \in\{1.8,1.9,1.10,2.9,2.12,2.13,2.16,2.17,2.20,2.21,2.22,3.13\} ;
$$

- in Appendix A, written by J.-P. Demailly, the relation between global log canonical thresholds of smooth Fano varieties and the $\alpha$-invariants of smooth Fano varieties introduced by G. Tian in [179] for the study of the existence of Kähler-Einstein metrics has been studied;

- in Appendix B, we put Table 1 that contains the list of all smooth Fano threefolds together with the known values and bounds for their global log canonical thresholds.

We use a standard notation $D_{1} \sim D_{2}$ (resp., $D_{1} \sim_{\mathbb{Q}} D_{2}$ ) for the linearly equivalent (resp., $\mathbb{Q}$-linearly equivalent) divisors (resp., $\mathbb{Q}$-divisors). If a divisor (resp., a $\mathbb{Q}$-divisor) $D$ is linearly equivalent to a line bundle $\mathcal{L}$ (resp., is $\mathbb{Q}$-linearly equivalent to a divisor that is linearly equivalent to $\mathcal{L}$ ), we write $D \sim \mathcal{L}$ (resp., $D \sim \mathbb{Q} \mathcal{L}$ ). Recall that $\mathbb{Q}$-linear equivalence coincides with numerical equivalence in the case of Fano varieties.

A divisor $D$ on $\mathbb{P}^{n_{1}} \times \mathbb{P}^{n_{2}} \times \ldots \times \mathbb{P}^{n_{m}}$ is said to be of multidegree $\left(a_{1}, a_{2}, \ldots, a_{m}\right) \in \mathbb{Z}^{m}$ if $D \sim \sum_{i=1}^{m} a_{i} H_{i}$, where

$$
H_{i} \sim \pi_{i}^{*}\left(\mathcal{O}_{\mathbb{P}^{n_{i}}}\left(a_{i}\right)\right)
$$

is a pull-back of a hyperplane section of $\mathbb{P}^{n_{i}}$ under the projection $\pi_{i}: \mathbb{P}^{n_{1}} \times \mathbb{P}^{n_{2}} \times \ldots \times \mathbb{P}^{n_{m}} \rightarrow \mathbb{P}^{n_{i}}$ is a natural projection. A curve $C \subset \mathbb{P}^{n_{1}} \times \mathbb{P}^{n_{2}} \times \ldots \times \mathbb{P}^{n_{m}}$ is said to be of multidegree $\left(a_{1}, a_{2}, \ldots, a_{m}\right) \in \mathbb{Z}^{m}$ if

$$
\pi_{i}^{*}\left(\mathcal{O}_{\mathbb{P}^{n_{i}}}(1)\right) \cdot C=a_{i}
$$

for $i=1, \ldots, m$.

The projectivisation $\mathbb{P}_{Y}(\mathcal{E})$ of a vector bundle $\mathcal{E}$ on a variety $Y$ is the variety of hyperplanes in the fibers of $\mathcal{E}$. The symbol $\mathbb{F}_{n}$ denotes the Hirzebruch surface $\mathbb{P}\left(\mathcal{O}_{\mathbb{P}^{1}} \oplus \mathcal{O}_{\mathbb{P}^{1}}(n)\right)$.

We always refer to a smooth Fano threefold $X$ using the ordinal number $\beth(X)$ introduced in Table 1 .

We are very grateful to J.-P. Demailly for writing Appendix A, and to C. Boyer, A. Iliev, P. Jahnke, A.-S. Kaloghiros, A. G. Kuznetsov, J. Park and Yu. G. Prokhorov for useful discussions. The first author would like to express his gratitude to IHES (Bures-sur-Yvette, France) and MPIM (Bonn, Germany) for hospitality.

\section{Preliminaries}

Let $X$ be a variety with log terminal singularities. Let us consider a $\mathbb{Q}$-divisor

$$
B_{X}=\sum_{i=1}^{r} a_{i} B_{i},
$$

where $B_{i}$ is a prime Weil divisor on the variety $X$, and $a_{i}$ is an arbitrary non-negative rational number. Suppose that $B_{X}$ is a $\mathbb{Q}$-Cartier divisor such that $B_{i} \neq B_{j}$ for $i \neq j$.

Let $\pi: \bar{X} \rightarrow X$ be a birational morphism such that $\bar{X}$ is smooth (see [75]). Put

$$
B_{\bar{X}}=\sum_{i=1}^{r} a_{i} \bar{B}_{i},
$$


where $\bar{B}_{i}$ is a proper transform of the divisor $B_{i}$ on the variety $\bar{X}$. Then

$$
K_{\bar{X}}+B_{\bar{X}} \sim_{\mathbb{Q}} \pi^{*}\left(K_{X}+B_{X}\right)+\sum_{i=1}^{n} c_{i} E_{i},
$$

where $c_{i} \in \mathbb{Q}$, and $E_{i}$ is an exceptional divisor of the morphism $\pi$. Suppose that

$$
\left(\bigcup_{i=1}^{r} \bar{B}_{i}\right) \bigcup\left(\bigcup_{i=1}^{n} E_{i}\right)
$$

is a divisor with simple normal crossing. Put

$$
B^{\bar{X}}=B_{\bar{X}}-\sum_{i=1}^{n} c_{i} E_{i} .
$$

Definition 2.1. The singularities of $\left(X, B_{X}\right)$ are log canonical (resp., log terminal) if

- the inequality $a_{i} \leqslant 1$ holds (resp., the inequality $a_{i}<1$ holds),

- the inequality $c_{j} \geqslant-1$ holds (resp., the inequality $c_{j}>-1$ holds), for every $i=1, \ldots, r$ and $j=1, \ldots, n$.

One can show that Definition 2.1 does not depend on the choice of the morphism $\pi$. Put

$$
\operatorname{LCS}\left(X, B_{X}\right)=\left(\bigcup_{a_{i} \geqslant 1} B_{i}\right) \bigcup\left(\bigcup_{c_{i} \leqslant-1} \pi\left(E_{i}\right)\right) \subsetneq X,
$$

then $\operatorname{LCS}\left(X, B_{X}\right)$ is called the locus of log canonical singularities of the log pair $\left(X, B_{X}\right)$.

Definition 2.2. A proper irreducible subvariety $Y \subsetneq X$ is said to be a center of log canonical singularities of the log pair $\left(X, B_{X}\right)$ if one of the following conditions is satisfied:

- either the inequality $a_{i} \geqslant 1$ holds and $Y=B_{i}$,

- or the inequality $c_{i} \leqslant-1$ holds and $Y=\pi\left(E_{i}\right)$,

for some choice of the birational morphism $\pi: \bar{X} \rightarrow X$.

Let $\mathbb{L} \mathbb{C} \mathbb{S}\left(X, B_{X}\right)$ be the set of all centers of log canonical singularities of $\left(X, B_{X}\right)$. Then

$$
Y \in \mathbb{L} \mathbb{C S}\left(X, B_{X}\right) \Longrightarrow Y \subseteq \operatorname{LCS}\left(X, B_{X}\right)
$$

and $\mathbb{L} \mathbb{C S}\left(X, B_{X}\right)=\varnothing \Longleftrightarrow \operatorname{LCS}\left(X, B_{X}\right)=\varnothing \Longleftrightarrow$ the log pair $\left(X, B_{X}\right)$ is log terminal.

Remark 2.3. Let $\mathcal{H}$ be a linear system on $X$ that has no base points, let $H$ be a sufficiently general divisor in the linear system $\mathcal{H}$, and let $Y \subsetneq X$ be an irreducible subvariety. Put

$$
\left.Y\right|_{H}=\sum_{i=1}^{m} Z_{i}
$$

where $Z_{i} \subset H$ is an irreducible subvariety. It follows from Definition 2.2 (cf. Theorem 2.20) that

$$
Y \in \mathbb{L} \mathbb{C} S\left(X, B_{X}\right) \Longleftrightarrow\left\{Z_{1}, \ldots, Z_{m}\right\} \subseteq \mathbb{L} \mathbb{C S}\left(H,\left.B_{X}\right|_{H}\right)
$$

Example 2.4. Let $\alpha: V \rightarrow X$ be a blow up of a smooth point $O \in X$. Then

$$
B_{V} \sim_{\mathbb{Q}} \alpha^{*}\left(B_{X}\right)-\operatorname{mult}_{O}\left(B_{X}\right) E,
$$

where $\operatorname{mult}_{O}\left(B_{X}\right) \in \mathbb{Q}$, and $E$ is the exceptional divisor of the blow up $\alpha$. Then

$$
\operatorname{mult}_{O}\left(B_{X}\right)>1
$$

if the log pair $\left(X, B_{X}\right)$ is not log canonical at the point $O$. Put

$$
B^{V}=B_{V}+\left(\operatorname{mult}_{O}\left(B_{X}\right)-\operatorname{dim}(X)+1\right) E
$$

and suppose that $\operatorname{mult}_{O}\left(B_{X}\right) \geqslant \operatorname{dim}(X)-1$. Then $O \in \mathbb{L} \mathbb{C S}\left(X, B_{X}\right)$ if and only if

- either $E \in \mathbb{L} \mathbb{C} S\left(V, B^{V}\right)$, i. e. $\operatorname{mult}_{O}\left(B_{X}\right) \geqslant \operatorname{dim}(X)$,

- or there is a subvariety $Z \subsetneq E$ such that $Z \in \mathbb{L} \mathbb{C S}\left(V, B^{V}\right)$. 
The locus $\operatorname{LCS}\left(X, B_{X}\right) \subset X$ can be equipped with a scheme structure (see [132], [169]). Put

$$
\mathcal{I}\left(X, B_{X}\right)=\pi_{*}\left(\sum_{i=1}^{n}\left\lceil c_{i}\right\rceil E_{i}-\sum_{i=1}^{r}\left\lfloor a_{i}\right\rfloor \bar{B}_{i}\right)
$$

and let $\mathcal{L}\left(X, B_{X}\right)$ be a subscheme that corresponds to the ideal sheaf $\mathcal{I}\left(X, B_{X}\right)$.

Definition 2.5. For the log pair $\left(X, B_{X}\right)$, we say that

- the subscheme $\mathcal{L}\left(X, B_{X}\right)$ is the subscheme of log canonical singularities of $\left(X, B_{X}\right)$,

- the ideal sheaf $\mathcal{I}\left(X, B_{X}\right)$ is the multiplier ideal sheaf of $\left(X, B_{X}\right)$.

It follows from the construction of the subscheme $\mathcal{L}\left(X, B_{X}\right)$ that

$$
\operatorname{Supp}\left(\mathcal{L}\left(X, B_{X}\right)\right)=\operatorname{LCS}\left(X, B_{X}\right) \subset X .
$$

The following result is the Nadel-Shokurov vanishing theorem (see [169], [111, Theorem 9.4.8]).

Theorem 2.6. Let $H$ be a nef and big $\mathbb{Q}$-divisor on $X$ such that

$$
K_{X}+B_{X}+H \sim_{\mathbb{Q}} D
$$

for some Cartier divisor $D$ on the variety $X$. Then for every $i \geqslant 1$

$$
H^{i}\left(X, \mathcal{I}\left(X, B_{X}\right) \otimes D\right)=0 .
$$

Proof. It follows from the Kawamata-Viehweg vanishing theorem (see [105]) that

$$
R^{i} \pi_{*}\left(\pi^{*}\left(K_{X}+B_{X}+H\right)+\sum_{i=1}^{n}\left\lceil c_{i}\right\rceil E_{i}-\sum_{i=1}^{r}\left\lfloor a_{i}\right\rfloor \bar{B}_{i}\right)=0
$$

for every $i>0$. It follows from the equality of sheaves

$$
\pi_{*}\left(\pi^{*}\left(K_{X}+B_{X}+H\right)+\sum_{i=1}^{n}\left\lceil c_{i}\right\rceil E_{i}-\sum_{i=1}^{r}\left\lfloor a_{i}\right\rfloor \bar{B}_{i}\right)=\mathcal{I}\left(X, B_{X}\right) \otimes D
$$

and from the degeneration of a local-to-global spectral sequence that

$$
H^{i}\left(X, \mathcal{I}\left(X, B_{X}\right) \otimes D\right)=H^{i}\left(\bar{X}, \pi^{*}\left(K_{X}+B_{X}+H\right)+\sum_{i=1}^{n}\left\lceil c_{i}\right\rceil E_{i}-\sum_{i=1}^{r}\left\lfloor a_{i}\right\rfloor \bar{B}_{i}\right),
$$

for every $i \geqslant 0$. But for $i>0$, the cohomology group

$$
H^{i}\left(\bar{X}, \pi^{*}\left(K_{X}+B_{X}+H\right)+\sum_{i=1}^{n}\left\lceil c_{i}\right\rceil E_{i}-\sum_{i=1}^{r}\left\lfloor a_{i}\right\rfloor \bar{B}_{i}\right)
$$

is trivial by the Kawamata-Viehweg vanishing theorem (see [105]).

For every Cartier divisor $D$ on the variety $X$, let us consider the exact sequence of sheaves

$$
0 \longrightarrow \mathcal{I}\left(X, B_{X}\right) \otimes D \longrightarrow \mathcal{O}_{X}(D) \longrightarrow \mathcal{O}_{\mathcal{L}\left(X, B_{X}\right)}(D) \longrightarrow 0,
$$

and let us consider the corresponding exact sequence of cohomology groups

$$
H^{0}\left(\mathcal{O}_{X}(D)\right) \longrightarrow H^{0}\left(\mathcal{O}_{\mathcal{L}\left(X, B_{X}\right)}(D)\right) \longrightarrow H^{1}\left(\mathcal{I}\left(X, B_{X}\right) \otimes D\right) .
$$

Theorem 2.7. Suppose that $-\left(K_{X}+B_{X}\right)$ is nef and big. Then $\operatorname{LCS}\left(X, B_{X}\right)$ is connected.

Proof. Put $D=0$. Then it follows from Theorem 2.6 that the sequence

$$
\mathbb{C}=H^{0}\left(\mathcal{O}_{X}\right) \longrightarrow H^{0}\left(\mathcal{O}_{\mathcal{L}\left(X, B_{X}\right)}\right) \longrightarrow H^{1}\left(\mathcal{I}\left(X, B_{X}\right)\right)=0
$$

is exact. Thus, the locus

is connected.

$$
\operatorname{LCS}\left(X, B_{X}\right)=\operatorname{Supp}\left(\mathcal{L}\left(X, B_{X}\right)\right)
$$

Let us consider few elementary applications of Theorem 2.7 (cf. Example 1.18). 
Lemma 2.8. Suppose that $\operatorname{LCS}\left(X, B_{X}\right) \neq \varnothing$, where $X \cong \mathbb{P}^{n}$, and

$$
B_{X} \sim_{\mathbb{Q}}-\lambda K_{X}
$$

for some rational number $0<\lambda<n /(n+1)$. Then

- the inequality $\operatorname{dim}\left(\operatorname{LCS}\left(X, B_{X}\right)\right) \geqslant 1$ holds,

- the subscheme $\mathcal{L}\left(X, B_{X}\right)$ does not contain isolated zero-dimensional components.

Proof. Suppose that there is a point $O \in X$ such that

$$
\operatorname{LCS}\left(X, \lambda B_{X}\right)=O \cup \Sigma,
$$

where $\Sigma \subset X$ is a possibly empty subset such that $O \notin X$.

Let $H$ be a general line in $X \cong \mathbb{P}^{2}$. Then the locus

$$
\operatorname{LCS}\left(X, \lambda B_{X}+H\right)=O \cup H \cup \Sigma
$$

is disconnected. But the divisor $-\left(K_{X}+\lambda B_{X}+H\right)$ is ample, which contradicts Theorem 2.7

Lemma 2.9. Suppose that $\operatorname{LCS}\left(X, B_{X}\right) \neq \varnothing$, where $X \cong \mathbb{P}^{3}$, and

$$
B_{X} \sim_{\mathbb{Q}}-\lambda K_{X}
$$

for some rational number $0<\lambda<1 / 2$. Then $\mathbb{L} \mathbb{C S}\left(X, B_{X}\right)$ contains a surface.

Proof. Suppose that $\mathbb{L} \mathbb{C S}\left(X, B_{X}\right)$ contains no surfaces. Let $S \subset \mathbb{P}^{3}$ be a general plane. The locus

$$
\operatorname{LCS}\left(\mathbb{P}^{3}, B_{X}+S\right)
$$

is connected by Theorem 2.7. Then $\left(S,\left.B_{X}\right|_{S}\right)$ is not log terminal by Remark 2.3. But the locus

$$
\operatorname{LCS}\left(S,\left.B_{X}\right|_{S}\right)
$$

consists of finitely many points, which is impossible by Lemma 2.8 .

Lemma 2.10. Suppose that $\operatorname{LCS}\left(X, B_{X}\right) \neq \varnothing$, where $X$ is a smooth quadric threefold in $\mathbb{P}^{4}$, and

$$
B_{X} \sim_{\mathbb{Q}}-\lambda K_{X}
$$

for some rational number $0<\lambda<1 / 2$. Then $\mathbb{L} \mathbb{C S}\left(X, B_{X}\right)$ contains a surface.

Proof. Let $L \subset X$ be a general line, let $P_{1} \in L \ni P_{2}$ be two general points, let $H_{1}$ and $H_{2}$ be the hyperplane sections of $X$ that are tangent to $X$ at the points $P_{1}$ and $P_{2}$, respectively. Then

$$
\operatorname{LCS}\left(X, \lambda B_{X}+\frac{3}{4}\left(H_{1}+H_{2}\right)\right)=\operatorname{LCS}\left(X, \lambda B_{X}\right) \cup L
$$

is disconnected, which is impossible by Theorem 2.7 .

Remark 2.11. One can prove Lemmas 2.9, 2.10 and 2.29 using the following trick. Suppose that

$$
B_{X} \sim_{\mathbb{Q}}-\lambda K_{X}
$$

for some $\lambda \in \mathbb{Q}$ such that $0<\lambda<1 / 2$, where $X$ is either $\mathbb{P}^{3}$, or $\mathbb{P}^{1} \times \mathbb{P}^{2}$, or a smooth quadric threefold, and the set $\mathbb{L} \mathbb{C S}\left(X, B_{X}\right)$ contains no surfaces. Then

$$
\operatorname{LCS}\left(X, B_{X}\right) \subseteq \Sigma,
$$

where $\Sigma \subset X$ is a (possibly reducible) curve. For a general $\phi \in \operatorname{Aut}(X)$ we have

$$
\phi(\Sigma) \cap \Sigma=\varnothing,
$$

which implies that $\operatorname{LCS}\left(X, \phi\left(B_{X}\right)\right) \cap \operatorname{LCS}\left(X, B_{X}\right)=\varnothing$. But

$$
\operatorname{LCS}\left(X, \phi\left(B_{X}\right)+B_{X}\right)=\operatorname{LCS}\left(X, \phi\left(B_{X}\right)\right) \bigcup \operatorname{LCS}\left(X, B_{X}\right)
$$

whenever $\phi$ is sufficiently general. The latter contradicts Theorem 2.7 since $\lambda<1 / 2$.

Lemma 2.12. Suppose that $\operatorname{LCS}\left(X, B_{X}\right) \neq \varnothing$, where $X$ is a blow up of $\mathbb{P}^{3}$ in one point, and

$$
B_{X} \sim_{\mathbb{Q}}-\lambda K_{X}
$$

for some rational number $0<\lambda<1 / 2$. Then $\mathbb{L} \mathbb{C} S\left(X, B_{X}\right)$ contains a surface. 
Proof. Suppose that the set $\mathbb{L} \mathbb{C} \mathbb{S}\left(X, B_{X}\right)$ contains no surfaces. Let

$$
\alpha: X \longrightarrow \mathbb{P}^{3}
$$

be the blow up of a point, and let $E$ be the exceptional divisor of $\alpha$. In the case when

$$
\operatorname{LCS}\left(X, \lambda B_{X}\right) \nsubseteq E
$$

we can apply Lemma 2.9 to the pair $\left(\mathbb{P}^{3}, \alpha\left(B_{X}\right)\right)$ to get a contradiction. Hence $\operatorname{LCS}\left(X, B_{X}\right) \subseteq E$.

Let $H \subset \mathbb{P}^{3}$ a general hyperplane, and let $H_{1} \subset \mathbb{P}^{3} \supset H_{2}$ be general hyperplanes that pass through $\alpha(E)$. Denote by $\bar{H}, \bar{H}_{1}$ and $\bar{H}_{2}$ the proper transforms of these planes on $X$. Then

$$
\operatorname{LCS}\left(X, B_{X}+\frac{1}{2}\left(\bar{H}_{1}+\bar{H}_{2}+2 \bar{H}\right)\right)
$$

is disconnected, which is impossible by Theorem 2.7 .

Lemma 2.13. Suppose that $X$ is a cone in $\mathbb{P}^{4}$ over a smooth quadric surface, and

$$
B_{X} \sim_{\mathbb{Q}}-\lambda K_{X}
$$

for some rational number $0<\lambda<1 / 3$. Then $\mathbb{L} \mathbb{C S}\left(X, B_{X}\right)=\varnothing$.

Proof. Suppose that $\mathbb{L} \mathbb{C S}\left(X, B_{X}\right) \neq \varnothing$. Let $S \subset X$ be a general hyperplane section. Then

$$
\operatorname{LCS}\left(S,\left.B_{X}\right|_{S}\right)=\varnothing
$$

because $S \cong \mathbb{P}^{1} \times \mathbb{P}^{1}$ and $\operatorname{lct}\left(\mathbb{P}^{1} \times \mathbb{P}^{1}\right)=1 / 2$ (see Example 1.18).

One has $\left|\operatorname{LCS}\left(X, B_{X}\right)\right|<+\infty$ by Remark 2.3 . Then the locus

$$
\operatorname{LCS}\left(X, B_{X}+S\right)
$$

is disconnected, which contradicts Theorem 2.7

The following result is a corollary Theorem 2.6 (see [132, Theorem 4.1]).

Lemma 2.14. Suppose that $-\left(K_{X}+B_{X}\right)$ is nef and big and $\operatorname{dim}\left(\operatorname{LCS}\left(X, B_{X}\right)\right)=1$. Then

- the locus $\operatorname{LCS}\left(X, B_{X}\right)$ is a connected union of smooth rational curves,

- every two irreducible components of the locus $\operatorname{LCS}\left(X, B_{X}\right)$ meet in at most one point,

- every intersecting irreducible components of the locus $\operatorname{LCS}\left(X, B_{X}\right)$ meet transversally,

- no three irreducible components of the locus $\operatorname{LCS}\left(X, B_{X}\right)$ meet in one point,

- the locus $\operatorname{LCS}\left(X, B_{X}\right)$ does not contain a cycle of smooth rational curves.

Proof. Arguing as in the proof of Theorem 2.7, we see that the locus $\operatorname{LCS}\left(X, B_{X}\right)$ is a connected tree of smooth rational curves with simple normal crossings.

To consider another application of Theorem [2.7, we need the following result (see [150], [21]).

Lemma 2.15. Suppose that $X$ is a smooth complete intersection $\cap_{i=1}^{k} G_{i} \subset \mathbb{P}^{m}$, and

$$
\left.B_{X} \sim_{\mathbb{Q}} \mathcal{O}_{\mathbb{P} m}(1)\right|_{X}
$$

where $G_{i}$ is a hypersurface. Let $S \subsetneq X$ be an irreducible subvariety such that $\operatorname{dim}(S) \geqslant k$. Then

$$
\operatorname{mult}_{S}\left(B_{X}\right) \leqslant 1 \text {. }
$$

Proof. We may assume $\operatorname{dim}(S)=k \leqslant(m-1) / 2$. Let $P$ be a sufficiently general point in $\mathbb{P}^{m}$, and let $C \subset \mathbb{P}^{m}$ be a cone over the subvariety $S$ with vertex in the point $P$. Then

$$
C \cap X=S \cup R,
$$

where $R$ is a curve on $X$. Let us calculate $|R \cap S|$. Let

$$
\pi: X \longrightarrow \mathbb{P}^{m-1}, \pi_{1}: G_{1} \longrightarrow \mathbb{P}^{m-1}, \ldots, \pi_{k}: G_{k} \longrightarrow \mathbb{P}^{m-1}
$$

be projections from the point $P$, let $D \subset X$ and $D_{i} \subset G_{i}$ be the ramification subvarieties of the projections $\pi$ and $\pi_{i}$, respectively. Put $C \cap G_{i}=S \cup R_{i}$. Then

$$
R_{i} \cap S=D_{i} \cap S
$$


by [155, Lemma 3]. Hence, it follows from $R=\cap_{i=1}^{k} R_{i}$ and $D=\cap_{i=1}^{k} D_{i}$ that $R \cap S=D \cap S$.

Let $\left(z_{0}, \ldots, z_{m}\right)$ be homogeneous coordinates on $\mathbb{P}^{m}$ such that

$$
P=\left(p_{0}, \ldots, p_{m}\right),
$$

and $G_{i} \subset \mathbb{P}^{m}$ is given by the equation $F_{i}\left(z_{0}, \ldots, z_{m}\right)=0$. Then $D \subset X$ is cut out by

$$
\sum_{i=0}^{m} \frac{\partial F_{1}\left(z_{0}, \ldots, z_{m}\right)}{\partial z_{i}} p_{i}=\sum_{i=0}^{m} \frac{\partial F_{2}\left(z_{0}, \ldots, z_{m}\right)}{\partial z_{i}} p_{i}=\ldots=\sum_{i=0}^{m} \frac{\partial F_{k}\left(z_{0}, \ldots, z_{m}\right)}{\partial z_{i}} p_{i}=0 .
$$

Let $\mathcal{F}_{r}$ be a linear system on $\mathbb{P}^{m}$ that contains divisors

$$
\sum_{i=0}^{m} \lambda_{i} \frac{\partial F_{r}\left(z_{0}, \ldots, z_{m}\right)}{\partial z_{i}}=0, r=1, \ldots, k .
$$

The variety $X$ is smooth. Hence

$$
|R \cap S|=|D \cap S|=\operatorname{deg}(S) \prod_{i=1}^{k}\left(\operatorname{deg}\left(F_{i}\right)-1\right),
$$

because $\mathcal{F}_{1}, \ldots, \mathcal{F}_{k}$ do not base points on $X$. Therefore, we have the inequality

$$
\operatorname{deg}(S) \prod_{i=1}^{k}\left(\operatorname{deg}\left(F_{i}\right)-1\right)=B_{X} \cdot R \geqslant \sum_{O \in R \cap S} \operatorname{mult}_{S}\left(B_{X}\right)=\operatorname{mult}_{S}\left(B_{X}\right)|R \cap S|,
$$

which implies $\operatorname{mult}_{S}\left(B_{X}\right) \leqslant 1$.

Using Remark 2.3, Theorem 2.7 and Lemma 2.15, we obtain the following result.

Corollary 2.16. Let $X$ is a smooth complete intersection $\cap_{i=1}^{k} G_{i} \subset \mathbb{P}^{m}$ such that

$$
m+1-\sum_{i=1}^{k} \operatorname{deg}\left(G_{i}\right) \geqslant k+1,
$$

where $G_{i}$ is a hypersurface in $\mathbb{P}^{m}$. Then $X$ is a Fano variety and

$$
\operatorname{lct}(X)=\frac{1}{m+1-\sum_{i=1}^{k} \operatorname{deg}\left(G_{i}\right)} .
$$

Let us consider another simple application of Theorem 2.7 and Lemma 2.15.

Lemma 2.17. Let $X$ be a cubic hypersurface in $\mathbb{P}^{4}$ such that $|\operatorname{Sing}(X)|<+\infty$. Suppose that

$$
B_{X} \sim \mathbb{Q}-K_{X},
$$

and there is a positive rational number $\lambda<1 / 2$ such that $\operatorname{LCS}\left(X, \lambda B_{X}\right) \neq \varnothing$. Then

$$
\operatorname{LCS}\left(X, \lambda B_{X}\right)=L,
$$

where $L$ is a line in $X \subset \mathbb{P}^{4}$ such that $L \cap \operatorname{Sing}(X) \neq \varnothing$.

Proof. Let $S$ be a general hyperplane section of $X$. Then

$$
S \cup \operatorname{LCS}\left(X, \lambda B_{X}\right) \subseteq \operatorname{LCS}\left(X, \lambda B_{X}+S\right),
$$

which implies that $\operatorname{dim}\left(\operatorname{LCS}\left(X, \lambda B_{X}\right)\right) \geqslant 1$ by Theorem 2.7. Then

$$
\operatorname{LCS}\left(S,\left.\lambda B_{X}\right|_{S}\right) \neq \varnothing
$$

by Remark 2.3. But $\left|\operatorname{LCS}\left(S,\left.\lambda B_{X}\right|_{S}\right)\right|<+\infty$ by Lemma 2.15. There is a point $O \in S$ such that

$$
\operatorname{LCS}\left(S,\left.\lambda B_{X}\right|_{S}\right)=O
$$

by Theorem 2.7. Therefore, there is a line $L \subset X$ such that $\operatorname{LCS}\left(X, \lambda B_{X}\right)=L$ by Remark 2.3 ,

Arguing as in the proof of Lemma 2.15, we see that $L \cap \operatorname{Sing}(X) \neq \varnothing$.

Similar to Lemma 2.17, one can prove the following result. 
Lemma 2.18. Suppose that there is a double cover $\tau: X \rightarrow \mathbb{P}^{3}$ branched over an irreducible reduced quartic surface $R \subset \mathbb{P}^{3}$ that has at most ordinary double points, the equivalence

$$
B_{X} \sim_{\mathbb{Q}}-\lambda K_{X}
$$

holds and $\operatorname{LCS}\left(X, B_{X}\right) \neq \varnothing$, where $\lambda<1 / 2$. Then $\operatorname{Sing}(X) \neq \varnothing$ and

$$
\operatorname{LCS}\left(X, B_{X}\right)=L
$$

where $L$ is an irreducible curve on $X$ such that $-K_{X} \cdot L=2$ and $L \cap \operatorname{Sing}(X) \neq \varnothing$.

Proof. We have $-K_{X} \sim 2 H$, where $H$ is a Cartier divisor on $X$ such that

$$
H \sim \tau^{*}\left(\mathcal{O}_{\mathbb{P}^{3}}(1)\right) .
$$

The variety $X$ is a Fano threefold, and $H^{3}=2$. Then

$$
\operatorname{LCS}\left(X, B_{X}+H\right)
$$

must be connected by Theorem 2.7. Thus, there is a curve

$$
C \in \mathbb{L} \mathbb{C} \mathbb{S}\left(X, B_{X}\right),
$$

which implies that $\operatorname{mult}_{C}\left(B_{X}\right) \geqslant 1 / \lambda>2$.

Let $S$ be a general surface in $|H|$. Put $B_{S}=\left.B_{X}\right|_{S}$. Then

$$
-\left.K_{S} \sim H\right|_{S} \sim_{\mathbb{Q}} \frac{1}{\lambda} B_{S}
$$

but the log pair $\left(S, B_{S}\right)$ is not log canonical in every point of the intersection $S \cap \operatorname{LCS}\left(X, B_{X}\right)$.

The surface $H$ is a smooth surface in $\mathbb{P}(1,1,1,2)$ of degree 4 .

Let $P$ be any point in $S \cap \operatorname{LCS}\left(X, B_{X}\right)$. Then there is a birational morphism

$$
\rho: S \longrightarrow \bar{S}
$$

such that $\bar{S}$ is a cubic surface in $\mathbb{P}^{3}$ and $\rho$ is an isomorphism in a neighborhood of $P$. Then

$$
\left(\bar{S}, \rho\left(B_{S}\right)\right)
$$

is not $\log$ terminal at the point $\rho(P)$. Thus, we have $\operatorname{LCS}\left(\bar{S}, \rho\left(B_{S}\right)\right) \neq \varnothing$. But

$$
\frac{1}{\lambda} \rho\left(B_{S}\right) \sim_{\mathbb{Q}}-\left.K_{\bar{S}} \sim \mathcal{O}_{\mathbb{P}^{3}}(1)\right|_{\bar{S}},
$$

which implies that $\operatorname{LCS}\left(\bar{S}, \rho\left(B_{S}\right)\right)$ consists of one point by Lemma 2.15 and Theorem 2.7. Then

$$
P=S \cap C=S \cap \operatorname{LCS}\left(X, B_{X}\right)
$$

if the point $P$ is sufficiently general. Therefore, we see that

$$
\operatorname{LCS}\left(X, B_{X}\right)=C \text {, }
$$

the curve $C$ is irreducible and $-K_{X} \cdot C=2$. Then $\tau(C) \subset \mathbb{P}^{3}$ is a line.

Suppose that $C \cap \operatorname{Sing}(X)=\varnothing$. Let us derive a contradiction.

Suppose that $\tau(C) \subset R$. Take a general point $O \in C$. Let

$$
\tau(O) \in \Pi \subset \mathbb{P}^{3}
$$

be a plane that is tangent to $R$ at the point $\tau(O)$. Arguing as in the proof of Lemma 2.15, we see that $\left.R\right|_{\Pi}$ is reduced along $\tau(C)$, because $\tau(C) \cap \operatorname{Sing}(R)=\varnothing$. Fix a general line

$$
\Gamma \subset \Pi \subset \mathbb{P}^{3}
$$

such that $\tau(O) \in \Gamma$. Let $\bar{\Gamma} \subset X$ be an irreducible curve such that $\tau(\bar{\Gamma})=\Gamma$. Then

$$
\bar{\Gamma} \not \operatorname{Supp}\left(B_{X}\right),
$$

because $\Gamma$ spans a dense subset in $\mathbb{P}^{3}$ when we vary the point $O \in C$ and the line $\Gamma \subset \Pi$. Note that $H \cdot \bar{\Gamma}$ equals either 1 or 2 , and $\operatorname{mult}_{O}(\bar{\Gamma})=2$ in the case when $H \cdot G a \bar{m} m a=2$. Hence

$$
H \cdot \bar{\Gamma}>2 \lambda H \cdot \bar{\Gamma}=\bar{\Gamma} \cdot B_{X} \geqslant \operatorname{mult}_{25}(\bar{\Gamma}) \operatorname{mult}_{C}\left(B_{V}\right) \geqslant H \cdot \bar{\Gamma},
$$


which is a contradiction. Thus, we see that $\tau(C) \not \subset R$.

There is an irreducible reduced curve $\bar{C} \subset X$ such that

$$
\tau(\bar{C})=\tau(C) \subset \mathbb{P}^{3}
$$

and $\bar{C} \neq C$. Let $Y$ be a general surface in $|H|$ that passes through the curves $\bar{C}$ and $C$. Then $Y$ is smooth, because $C \cap \operatorname{Sing}(X)=\varnothing$, and

$$
\bar{C} \cdot \bar{C}=C \cdot C=-2
$$

on the surface $Y$.

By construction, we have $Y \not \subset \operatorname{Supp}\left(B_{X}\right)$. Put $B_{Y}=\left.B_{X}\right|_{Y}$. Then

$$
B_{Y}=\operatorname{mult}_{\bar{C}}\left(B_{X}\right) \bar{C}+\operatorname{mult}_{C}\left(B_{X}\right) C+\Delta
$$

where $\Delta$ is an effective $\mathbb{Q}$-divisor on the surface $Y$ such that $\bar{C} \not \subset \operatorname{Supp}(\Delta) \not \supset C$. But

$$
B_{Y} \sim_{\mathbb{Q}} 2 \lambda(\bar{C}+C),
$$

which implies, in particular, that

$$
\left(2 \lambda-\operatorname{mult}_{C}\left(B_{X}\right)\right) C \cdot C=\left(\operatorname{mult}_{\bar{C}}\left(B_{X}\right)-2 \lambda\right) \bar{C} \cdot C+\Delta \cdot C \geqslant\left(\operatorname{mult}_{\bar{C}}\left(B_{X}\right)-2 \lambda\right) \bar{C} \cdot C \geqslant 0,
$$

because $\Delta \cdot C \geqslant 0$ and $\bar{C} \cdot C \geqslant 0$. Then $\operatorname{mult}_{\bar{C}}\left(B_{X}\right) \geqslant 2 \lambda$, because $C \cdot C<0$. Thus, we have

$$
-\Delta \sim_{\mathbb{Q}}\left(\operatorname{mult}_{\bar{C}}\left(B_{X}\right)-2 \lambda\right) \bar{C}+\left(\operatorname{mult}_{C}\left(B_{X}\right)-2 \lambda\right) C
$$

which is impossible, because $\operatorname{mult}_{C}\left(B_{X}\right)>2 \lambda$ and $Y$ is projective.

One can generalize Theorem 2.7 in the following way (see [169, Lemma 5.7]).

Theorem 2.19. Let $\psi: X \rightarrow Z$ be a morphism. Then the set

$$
\operatorname{LCS}\left(\bar{X}, B^{\bar{X}}\right)
$$

is connected in a neighborhood of every fiber of the morphism $\psi \circ \pi: X \rightarrow Z$ in the case when

- the morphism $\psi$ is surjective and has connected fibers,

- the divisor $-\left(K_{X}+B_{X}\right)$ is nef and big with respect to $\psi$.

Let us consider one important application of Theorem 2.19 (see [108, Theorem 5.50]).

Theorem 2.20. Suppose that $B_{1}$ is a Cartier divisor, $a_{1}=1$, and $B_{1}$ has at most log terminal singularities. Then the following assertions are equivalent:

- the log pair $\left(X, B_{X}\right)$ is log canonical in a neighborhood of the divisor $B_{1}$;

- the singularities of the log pair $\left(B_{1},\left.\sum_{i=2}^{r} a_{i} B_{i}\right|_{B_{1}}\right)$ are $\log$ canonical.

Proof. Suppose that the singularities of the log pair $\left(X, B_{X}\right)$ are not log canonical in a neighborhood of the divisor $B_{1} \subset X$. Let us show that $\left(B_{1},\left.\sum_{i=2}^{r} a_{i} B_{i}\right|_{B_{1}}\right)$ is not log canonical.

In the case when $a_{m}>1$ and $B_{m} \cap B_{1} \neq \varnothing$ for some $m \geqslant 2$, the log pair

$$
\left(B_{1},\left.\sum_{i=2}^{r} a_{i} B_{i}\right|_{B_{1}}\right)
$$

is not $\log$ canonical by Definition 2.1 . Thus, we may assume that $a_{i} \leqslant 1$ for every $i$. Then

$$
\left(X, B_{1}+\sum_{i=2}^{r} \lambda a_{i} B_{i}\right)
$$

is not $\log$ canonical as well for some rational number $\lambda<1$. Then

$$
K_{\bar{X}}+\bar{B}_{1}+\sum_{i=2}^{r} \lambda a_{i} \bar{B}_{i} \sim_{\mathbb{Q}} \pi^{*}\left(K_{X}+B_{1}+\sum_{i=2}^{r} \lambda a_{i} B_{i}\right)+\sum_{i=1}^{n} d_{i} E_{i}
$$

for some rational numbers $d_{1}, \ldots, d_{n}$. It follows from Theorem 2.19 that

$$
\bar{B}_{1} \cap E_{k} \neq \varnothing
$$


and the inequality $d_{k} \leqslant-1$ holds for some $k$. But

$$
K_{\bar{B}_{1}}+\left.\sum_{i=2}^{r} \lambda a_{i} \bar{B}_{i}\right|_{B_{1}} \sim_{\mathbb{Q}} \phi^{*}\left(K_{B_{1}}+\left.\sum_{i=2}^{r} \lambda a_{i} B_{i}\right|_{B_{1}}\right)+\left.\sum_{i=1}^{n} d_{i} E_{i}\right|_{B_{1}},
$$

where $\phi: \bar{B}_{1} \rightarrow B_{1}$ is a birational morphism that is induced by $\pi$.

Thus, the log pair $\left(B_{1},\left.\sum_{i=2}^{r} \lambda a_{i} B_{i}\right|_{B_{1}}\right)$ is not log terminal. Then the log pair

$$
\left(B_{1},\left.\sum_{i=2}^{r} a_{i} B_{i}\right|_{B_{1}}\right)
$$

is not log canonical. The rest of the proof is similar (see the proof of [105, Theorem 7.5]).

The simplest application of Theorem 2.20 is a non-obvious result (see [108, Corollary 5.57]).

Lemma 2.21. Suppose that $\operatorname{dim}(X)=2$ and $a_{1} \leqslant 1$. Then

$$
\left(\sum_{i=2}^{r} a_{i} B_{i}\right) \cdot B_{1}>1
$$

whenever $\left(X, B_{X}\right)$ is not $\log$ canonical at some point $O \in B_{1}$ such that $O \notin \operatorname{Sing}(X) \cup \operatorname{Sing}\left(B_{1}\right)$. Proof. Suppose that $\left(X, B_{X}\right)$ is not $\log$ canonical in a point $O \in B_{1}$. By Theorem 2.20, we have

$$
\left(\sum_{i=2}^{r} a_{i} B_{i}\right) \cdot B_{1} \geqslant \operatorname{mult}_{O}\left(\left.\sum_{i=2}^{r} a_{i} B_{i}\right|_{B_{1}}\right)>1
$$

if $O \notin \operatorname{Sing}(X) \cup \operatorname{Sing}\left(B_{1}\right)$, because $\left(X, B_{1}+\sum_{i=2}^{r} a_{i} B_{i}\right)$ is not $\log$ canonical at the point $O$.

Let us consider another application of Theorem 2.20 (cf. Lemma 2.30).

Lemma 2.22. Suppose that $X$ is a Fano variety with log terminal singularities. Then

$$
\operatorname{lct}\left(\mathbb{P}^{1} \times X\right)=\min \left(\frac{1}{2}, \operatorname{lct}(X)\right) .
$$

Proof. The inequalities $1 / 2 \geqslant \operatorname{lct}(V \times U) \leqslant \operatorname{lct}(X)$ are obvious. Suppose that

$$
\operatorname{lct}\left(\mathbb{P}^{1} \times X\right)<\min \left(\frac{1}{2}, \operatorname{lct}(X)\right),
$$

and let us show that this assumption leads to a contradiction.

There is an effective $\mathbb{Q}$-divisor $D \sim_{\mathbb{Q}}-K_{\mathbb{P}^{1} \times X}$ such that the log pair

$$
\left(\mathbb{P}^{1} \times X, \lambda D\right)
$$

is not $\log$ canonical in some point $P \in \mathbb{P}^{1} \times X$, where $\lambda<\min (1 / 2, \operatorname{lct}(X))$.

Let $F$ be a fiber of the projection $\mathbb{P}^{1} \times X \rightarrow \mathbb{P}^{1}$ such that $P \in F$. Then

$$
D=\mu F+\Omega,
$$

where $\Omega$ is an effective $\mathbb{Q}$-divisor on $\mathbb{P}^{1} \times X$ such that $F \not \subset \operatorname{Supp}(\Omega)$.

Let $L$ be a general fiber of the projection $\mathbb{P}^{1} \times X \rightarrow X$. Then

$$
2=D \cdot L=\mu+\Omega \cdot L \geqslant \mu,
$$

which implies that the log pair $\left(\mathbb{P}^{1} \times X, F+\lambda \Omega\right)$ is not log canonical at the point $P$. Then

$$
\left(F,\left.\lambda \Omega\right|_{F}\right)
$$

is not $\log$ canonical at the point $P$ by Theorem 2.20 , But

$$
\left.\left.\Omega\right|_{F} \sim_{\mathbb{Q}} D\right|_{F} \sim_{\mathbb{Q}}-K_{F},
$$

which is impossible, because $X \cong F$ and $\lambda<\operatorname{lct}(X)$. 
Let $P$ be a point in $X$. Let us consider an effective divisor

$$
\Delta=\sum_{i=1}^{r} \varepsilon_{i} B_{i} \sim_{\mathbb{Q}} B_{X},
$$

where $\varepsilon_{i}$ is a non-negative rational number. Suppose that

- the divisor $\Delta$ is a $\mathbb{Q}$-Cartier divisor,

- the equivalence $\Delta \sim \mathbb{Q} B_{X}$ holds,

- the log pair $(X, \Delta)$ is $\log$ canonical in the point $P \in X$.

Remark 2.23. Suppose that $\left(X, B_{X}\right)$ is not log canonical in the point $P \in X$. Put

$$
\alpha=\min \left\{\frac{a_{i}}{\varepsilon_{i}} \mid \varepsilon_{i} \neq 0\right\},
$$

where $\alpha$ is well defined, because there is $\varepsilon_{i} \neq 0$. Then $\alpha<1$, the log pair

$$
\left(X, \sum_{i=1}^{r} \frac{a_{i}-\alpha \varepsilon_{i}}{1-\alpha} B_{i}\right)
$$

is not $\log$ canonical in the point $P \in X$, the equivalence

$$
\sum_{i=1}^{r} \frac{a_{i}-\alpha \varepsilon_{i}}{1-\alpha} B_{i} \sim_{\mathbb{Q}} B_{X} \sim_{\mathbb{Q}} \Delta
$$

holds, and at least one irreducible component of the divisor $\operatorname{Supp}(\Delta)$ is not contained in

$$
\operatorname{Supp}\left(\sum_{i=1}^{r} \frac{a_{i}-\alpha \varepsilon_{i}}{1-\alpha} B_{i}\right) \text {. }
$$

The assertion of Remark 2.23 is obvious. Nevertheless it is very useful.

Lemma 2.24. Suppose that $X \cong C_{1} \times C_{2}$, where $C_{1}$ and $C_{2}$ are smooth curves, suppose that

$$
B_{X} \sim_{\mathbb{Q}} \lambda E+\mu F
$$

where $E \cong C_{1}$ and $F \cong C_{2}$ are curves on the surface $X$ such that

$$
E \cdot E=F \cdot F=0
$$

and $E \cdot F=1$, and $\lambda$ and $\mu$ are non-negative rational numbers. Then

- the pair $\left(X, B_{X}\right)$ is log terminal if $\lambda<1$ and $\mu<1$,

- the pair $\left(X, B_{X}\right)$ is $\log$ canonical if $\lambda \leqslant 1$ and $\mu \leqslant 1$.

Proof. Suppose that $\lambda, \mu<1$, but $\left(X, B_{X}\right)$ is not log terminal at some point $P \in X$. Then

$$
\operatorname{mult}_{P}\left(B_{X}\right) \geqslant 1 \text {, }
$$

and we may assume that $E \not \subset \operatorname{Supp}\left(B_{X}\right)$ or $F \not \subset \operatorname{Supp}\left(B_{X}\right)$ by Remark 2.23. But

$$
E \cdot B_{X}=\mu, F \cdot B_{X}=\lambda,
$$

which immediately leads to a contradiction, because $\operatorname{mult}_{P}\left(B_{X}\right) \geqslant 1$.

Let $\left[B_{X}\right]$ be a class of $\mathbb{Q}$-rational equivalence of the divisor $B_{X}$. Put

$$
\operatorname{lct}\left(X,\left[B_{X}\right]\right)=\inf \left\{\operatorname{lct}(X, D) \mid D \text { is an effective } \mathbb{Q} \text {-divisor on } X \text { such that } D \sim_{\mathbb{Q}} B_{X}\right\} \geqslant 0 \text {, }
$$

and put $\operatorname{lct}\left(X,\left[B_{X}\right]\right)=+\infty$ if $B_{X}=0$. Note that $B_{X}$ is an effective by assumption.

Remark 2.25. The equality $\operatorname{lct}\left(X,\left[-K_{X}\right]\right)=\operatorname{lct}(X)$ holds (see Definition 1.7).

Arguing as in the proof of Lemma 2.22, we obtain the following result. 
Lemma 2.26. Suppose that there is a surjective morphism with connected fibers

$$
\phi: X \longrightarrow Z
$$

such that $\operatorname{dim}(Z)=1$. Let $F$ be a fiber of $\phi$ that has $\log$ terminal singularities. Then either

$$
\operatorname{lct}_{F}\left(X, B_{X}\right) \geqslant \operatorname{lct}\left(F,\left[\left.B_{X}\right|_{F}\right]\right),
$$

or there is a positive rational number $\varepsilon<\operatorname{lct}\left(F,\left[\left.B_{X}\right|_{F}\right]\right)$ such that $F \subseteq \operatorname{LCS}\left(X, \varepsilon B_{X}\right)$.

Proof. Suppose that $\operatorname{lct}_{F}\left(X, B_{X}\right)<\operatorname{lct}\left(F,\left[\left.B_{X}\right|_{F}\right]\right)$. Then there is a rational number

$$
\varepsilon<\operatorname{lct}\left(F,\left[\left.B_{X}\right|_{F}\right]\right)
$$

such that the $\log$ pair $\left(X, \varepsilon B_{X}\right)$ is not $\log$ canonical at some point $P \in F$. Put

$$
B_{X}=\mu F+\Omega \text {, }
$$

where $\Omega$ is an effective $\mathbb{Q}$-divisor on $X$ such that $F \not \subset \operatorname{Supp}(\Omega)$.

We may assume that $\varepsilon \mu \leqslant 1$. Then $(X, F+\varepsilon \Omega)$ is not canonical at the point $P$. Then

$$
\left(F,\left.\varepsilon \Omega\right|_{F}\right)
$$

is not $\log$ canonical at $P$ by Theorem 2.20 . But $\left.\left.\Omega\right|_{F} \sim_{\mathbb{Q}} B_{X}\right|_{F}$, which is a contradiction.

Let us show how to apply Lemma 2.26.

Lemma 2.27. Let $Q \subset \mathbb{P}^{4}$ be a cone over a smooth quadric surface, and let $\alpha: X \rightarrow Q$ be a blow up along a smooth conic $C \subset Q \backslash \operatorname{Sing}(Q)$. Then $\operatorname{lct}(X)=1 / 3$.

Proof. Let $H$ be a general hyperplane section of $Q \subset \mathbb{P}^{4}$ that contains $C$, and let $\bar{H}$ be a proper transform of the surface $H$ on the threefold $X$. Then

$$
-K_{X} \sim 3 \bar{H}+2 E,
$$

where $E$ is the exceptional divisor of $\alpha$. In particular, the inequality $\operatorname{lct}(X) \leqslant 1 / 3$ holds.

We suppose that $\operatorname{lct}(X)<1 / 3$. Then there exists an effective $\mathbb{Q}$-divisor $D \sim_{\mathbb{Q}}-K_{X}$ such that the log pair $(X, \lambda D)$ is not log canonical for some positive rational number $\lambda<1 / 3$.

There is a commutative diagram

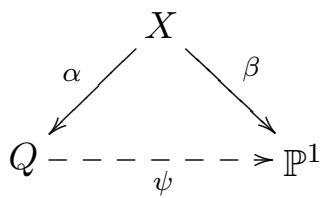

where $\beta$ is a morphism given by the linear system $|\bar{H}|$, and $\psi$ is a projection from the twodimensional linear subspace that contains the conic $C$.

Suppose that $\mathbb{L} \mathbb{C} S(X, \lambda D)$ contains a surface $M \subset X$. Then

$$
D=\mu M+\Omega,
$$

where $\mu \geqslant 1 / \lambda$, and $\Omega$ is an effective $\mathbb{Q}$-divisor such that $M \not \subset \operatorname{Supp}(\Omega)$.

Let $F$ be a general fiber of $\beta$. Then $F \cong \mathbb{P}^{1} \times \mathbb{P}^{1}$ and

$$
\left.D\right|_{F}=\left.\mu M\right|_{F}+\left.\Omega\right|_{F} \sim_{\mathbb{Q}}-K_{F},
$$

which immediately implies that $M$ is a fiber of the morphism $\beta$. But

$$
\alpha(D)=\mu \alpha(M)+\alpha(\Omega) \sim_{\mathbb{Q}}-K_{Q} \sim 3 \alpha(M),
$$

which is impossible, because $\mu \geqslant 1 / \lambda>3$. Thus, the set $\mathbb{L} \mathbb{C} \mathbb{S}(X, \lambda D)$ contains no surfaces.

There is a fiber $S$ of the morphism $\beta$ such that

$$
S \neq S \cap \operatorname{LCS}(X, \lambda D) \neq \varnothing,
$$

which implies that $S$ is singular by Lemma 2.26 , because $\operatorname{lct}\left(\mathbb{P}^{1} \times \mathbb{P}^{1}\right)=1 / 2$. 
Thus, the surface $S$ is an irreducible quadric cone in $\mathbb{P}^{3}$. Then

$$
\operatorname{LCS}(X, \lambda D) \subseteq S
$$

by Theorem 2.7. We may assume that either $S \not \subset \operatorname{Supp}(D)$ or $E \not \subset \operatorname{Supp}(D)$ by Remark 2.23 , because

$$
\left(X, S+\frac{2}{3} E\right)
$$

has $\log$ canonical singularities, and the equivalence $3 S+2 E \sim_{\mathbb{Q}} D$ holds.

Put $\Gamma=E \cap S$. The curve $\Gamma$ is an irreducible conic in $S$. Then

$$
\operatorname{LCS}(X, \lambda D) \subseteq \Gamma
$$

by Lemma 2.13, Intersecting $D$ with a general ruling of the cone $S \subset \mathbb{P}^{3}$, and intersecting $D$ with a general fiber of the projection $E \rightarrow C$, we see that

$$
\Gamma \nsubseteq \operatorname{LCS}(X, \lambda D)
$$

which implies that $\operatorname{LCS}(X, \lambda D)$ consists of a single point $O \in \Gamma$ by Theorem 2.7

Let $R$ be a general (not passing through $O$ ) surface in $\left|\alpha^{*}(H)\right|$. Then

$$
\operatorname{LCS}\left(X, \lambda D+\frac{1}{2}(\bar{H}+2 R)\right)=R \cup O,
$$

which is impossible by Theorem 2.7, since $-K_{X} \sim \bar{H}+2 R \sim_{\mathbb{Q}} D$ and $\lambda<1 / 3$.

The following generalization of Lemma 2.26 is proved in [79].

Theorem 2.28. Suppose that there is a surjective morphism with connected fibers

$$
\phi: X \longrightarrow Z
$$

such that $\phi$ is smooth in a neighborhood of a fiber $F$ of the morphism $\phi$. Then either

$$
\operatorname{lct}_{F}\left(X, B_{X}\right) \geqslant \operatorname{lct}\left(F,\left[\left.B_{X}\right|_{F}\right]\right) \text {, }
$$

or the equality $\operatorname{lct}_{O}\left(X, B_{X}\right)=\operatorname{lct}_{Q}\left(X, B_{X}\right)$ holds for every two points $O \in F \ni Q$.

Let us consider two elementary applications of Theorem 2.28

Lemma 2.29. Suppose that $\operatorname{LCS}\left(X, B_{X}\right) \neq \varnothing$, where $X \cong \mathbb{P}^{1} \times \mathbb{P}^{2}$ and

$$
B_{X} \sim_{\mathbb{Q}}-\lambda K_{X}
$$

for some rational number $0<\lambda<1 / 2$. Then $\mathbb{L} \mathbb{C} S\left(X, B_{X}\right)$ contains a surface.

Proof. Suppose that $\mathbb{L} \mathbb{C S}\left(X, B_{X}\right)$ contains no surfaces. By Theorems 2.7 and 2.28, we have

$$
\operatorname{LCS}\left(X, B_{X}\right)=F,
$$

where $F$ is a fiber of the natural projection $\pi_{2}: X \rightarrow \mathbb{P}^{2}$. Let $S$ be a general surface in

$$
\left|\pi_{1}^{*}\left(\mathcal{O}_{\mathbb{P}^{2}}(1)\right)\right|,
$$

let $M_{1}$ and $M_{2}$ be general fibers of the natural projection $\pi_{1}: X \rightarrow \mathbb{P}^{1}$. Then the locus

$$
\operatorname{LCS}\left(X, \lambda D+\frac{1}{2}\left(M_{1}+M_{2}+3 S\right)\right)=F \cup S
$$

is disconnected, which is impossible by Theorem 2.7 .

Lemma 2.30. Let $V$ and $U$ be smooth Fano varieties. Then

$$
\operatorname{lct}(V \times U)=\min (\operatorname{lct}(V), \operatorname{lct}(U)) .
$$


Proof. The inequalities $\operatorname{lct}(U) \geqslant \operatorname{lct}(V \times U) \leqslant \operatorname{lct}(U)$ are obvious. Suppose that

$$
\operatorname{lct}(V \times U)<\min (\operatorname{lct}(V), \operatorname{lct}(U)) \text {, }
$$

and let us show that this assumption leads to a contradiction.

There is an effective $\mathbb{Q}$-divisor $D \sim_{\mathbb{Q}}-K_{V \times U}$ such that the log pair

$$
(V \times U, \lambda D)
$$

is not $\log$ canonical in some point $P \in V \times U$, where $\lambda<\min (\operatorname{lct}(V)$, $\operatorname{lct}(U))$.

Let us identify $V$ with a fiber of the projection $V \times U \rightarrow U$ that contains the point $P$. Then

$$
\operatorname{lct}_{O}(V \times U, D)=\operatorname{lct}_{Q}(V \times U, D)
$$

for every points $O \in V \ni Q$ by Theorem 2.28, because the inequalities

$$
\operatorname{lct}(V)>\lambda>\operatorname{lct}_{V}(V \times U, D) \geqslant \operatorname{lct}\left(V,\left[\left.D\right|_{V}\right]\right)=\operatorname{lct}\left(V,\left[-K_{V}\right]\right)=\operatorname{lct}(V)
$$

are inconsistent. So, the log pair $(V \times U, \lambda D)$ is not log canonical in every point of $V \subset V \times U$.

Let us identify $U$ with a general fiber of the projection $V \times U \rightarrow V$. Then

$$
\left.D\right|_{U} \sim_{\mathbb{Q}}-K_{U}
$$

and $\left(U,\left.\lambda D\right|_{U}\right)$ is not log canonical in $U \cap V$ by Remark 2.3 (applied $\operatorname{dim}(V)$ times here), which contradicts the inequality $\lambda<\operatorname{lct}(U)$.

We believe that the assertion of Lemma 2.30 holds for log terminal varieties (cf. Lemma 2.22).

\section{The Mukai-Umemura threefold}

The main purpose of this section is to compute the global log canonical threshold of one remarkable smooth Fano threefold (cf. [55]) to illustrate the proof of Theorem 1.78 .

Lemma 3.1. Let $X$ be the smooth Fano threefold such that

$$
\operatorname{Pic}(X)=\mathbb{Z}\left[-K_{X}\right]
$$

the equality $-K_{X}^{3}=22$ holds, and $\operatorname{Aut}(X) \cong \operatorname{PSL}(2, \mathbb{C})$. Then $\operatorname{lct}(X)=1 / 2$.

Proof. Let $U \subset \mathbb{C}[x, y]$ be a subspace of forms of degree 12 . Consider $U \cong \mathbb{C}^{13}$ as the affine part of

$$
\mathbb{P}(U \oplus \mathbb{C}) \cong \mathbb{P}^{13}
$$

and let us identify $\mathbb{P}(U)$ with the hyperplane at infinity.

The natural action of $\operatorname{SL}(2, \mathbb{C})$ on $\mathbb{C}[x, y]$ induces an action on $\mathbb{P}(U \oplus \mathbb{C})$. Put

$$
\phi=x y\left(x^{10}-11 x^{5} y^{5}-y^{10}\right) \in U
$$

and consider the closure $\overline{\mathrm{SL}(2, \mathbb{C}) \cdot[\phi+1]} \subset \mathbb{P}(U \oplus \mathbb{C})$. It follows from [130] that

$$
X \cong \overline{\mathrm{SL}(2, \mathbb{C}) \cdot[\phi+1]}
$$

and the embedding $X \subset \mathbb{P}(U \oplus \mathbb{C}) \cong \mathbb{P}^{13}$ is induced by $\left|-K_{X}\right|$.

The action of $\operatorname{SL}(2, \mathbb{C})$ on $X$ has the following orbits (see [98, Theorem 5.2.13]):

- the three-dimensional orbit $\Sigma_{3}=\mathrm{SL}(2, \mathbb{C}) \cdot[\phi+1]$;

- the two-dimensional orbit $\Sigma_{2}=\operatorname{SL}(2, \mathbb{C}) \cdot\left[x y^{11}\right]$;

- the one-dimensional orbit $\Sigma_{1}=\mathrm{SL}(2, \mathbb{C}) \cdot\left[y^{12}\right]$.

The orbit $\Sigma_{3}$ is open, the orbit $\Sigma_{1} \cong \mathbb{P}^{1}$ is closed, and

$$
\bar{\Sigma}_{2}=\Sigma_{1} \cup \Sigma_{2} \text {, }
$$

so that the orbit $\Sigma_{2}$ is neither open nor closed. One has

$$
X \cap \mathbb{P}(U)=\Sigma_{1} \cup \Sigma_{2}
$$

and $X=\Sigma_{1} \cup \Sigma_{2} \cup \Sigma_{3}$. Put $R=X \cap \mathbb{P}(U)$. It follows from [130] that

\footnotetext{
${ }^{4}$ The threefold $X$ satisfying these assumptions is unique (see [130] and [142]).
} 
- the surface $R$ is swept out by lines on $X \subset \mathbb{P}^{13}$,

- the surface $R$ contains all lines on $X \subset \mathbb{P}^{13}$,

- for any lines $L_{1} \subset R \supset L_{2}$ such that $L_{1} \neq L_{2}$, one has $L_{1} \cap L_{2}=\varnothing$,

- the surface $R$ is singular along the orbit $\Sigma_{1} \cong \mathbb{P}^{1}$,

- the normalization of the surface $R$ is isomorphic to $\mathbb{P}^{1} \times \mathbb{P}^{1}$,

- for every point $P \in \Sigma_{1}$, the surface $R$ is locally isomorphic to

$$
x^{2}=y^{3} \subset \mathbb{C}^{3} \cong \operatorname{Spec}(\mathbb{C}[x, y, z]),
$$

which implies that $\operatorname{lct}(X, R)=5 / 6$.

The structure of the surface $R$ can be seen as follows. We see that

$$
\Sigma_{2}=\left\{\left[(a x+b y)(c x+d y)^{11}\right] \mid a d-b c=1\right\} \subset \mathbb{P}(U),
$$

which implies that there is a birational morphism $\nu: \mathbb{P}^{1} \times \mathbb{P}^{1} \rightarrow R$ that is defined by

$$
\nu:[a: b] \times[c: d] \mapsto\left[(a x+b y)(c x+d y)^{11}\right] \in R,
$$

so that $\nu$ is a normalization of the surface $R$.

Let $V_{5}$ be a smooth Fano threefold such that

$$
-K_{V_{5}} \sim 2 H
$$

and $H^{3}=5$, where $H$ is a Cartier divisor on $V_{5}$. Then $|H|$ induces an embedding $X \subset \mathbb{P}^{6}$.

Let $L \cong \mathbb{P}^{1}$ be a line on $X$. Then $\mathcal{N}_{L / X} \cong \mathcal{O}_{\mathbb{P}^{1}}(-2) \oplus \mathcal{O}_{\mathbb{P}^{1}}(1)$. Let

$$
\alpha_{L}: U_{L} \longrightarrow X
$$

be a blow up of the line $L$, and let $E_{L}$ be the exceptional divisor of $\alpha_{L}$. Then

$$
E_{L} \cong \mathbb{F}_{3},
$$

and it follows from Theorem 4.3.3 in [98] (see [45], [178]) that there is a commutative diagram

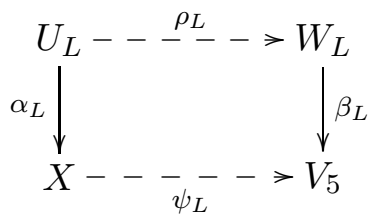

where $\rho_{L}$ is a flop in the exceptional section of $E \cong \mathbb{F}_{3}$, the morphism $\beta_{L}$ contracts a surface $D_{L} \subset W_{L}$ to a smooth rational curve of degree 5 , and $\psi_{L}$ is a double projection from the line $L$.

Let $\bar{D}_{L} \subset X$ be the proper transform of the surface $D_{L}$. Then

$$
\operatorname{mult}_{L}\left(\bar{D}_{L}\right)=3
$$

and $\bar{D}_{L} \sim-K_{X}$. It follows from [63] that $X \backslash \bar{D}_{L} \cong \mathbb{C}^{3}$ (cf. [138], [139], 65]).

It follows from 64] that there is an open subset $\breve{D}_{L} \subset \bar{D}_{L}$ that is given by

$\mu_{0} x^{4}+\left(\mu_{1} y z+\mu_{2} z^{3}\right) x^{3}+\left(\mu_{3} y^{3}+\mu_{4} y^{2} z^{2}+\mu_{5} y z^{4}\right) x^{2}+\left(\mu_{6} y^{4} z+\mu_{7} y^{3} z^{3}\right) x+\mu_{8} y^{6}+\mu_{9} y^{5} z^{2}=0$ in $\mathbb{C}^{3} \cong \operatorname{Spec}(\mathbb{C}[x, y, z])$, where $L \cap \Sigma_{1} \in \breve{D}_{L}$ is given by the equations $x=y=z=0$, and

$$
\begin{aligned}
& \mu_{0}=-2^{8} 5^{2}, \mu_{1}=2^{9} 3^{3} 5, \mu_{2}=-2^{6} 3^{4} 5, \mu_{3}=-2^{8} 3^{3} 7, \mu_{4}=-2^{4} 3^{4} 127, \\
& \mu_{5}=2^{9} 3^{5}, \mu_{6}=2^{2} 3^{6} 89, \mu_{7}=-2^{8} 3^{6}, \mu_{8}=-3^{6} 5^{3}, \mu_{9}=2^{5} 3^{7} .
\end{aligned}
$$

Put $O_{L}=\Sigma_{1} \cap L$. Then mult $O_{L}\left(\bar{D}_{L}\right)=4$, and it follows from Proposition 8.14 in [105] that

$$
\operatorname{LCS}\left(X, \frac{1}{2} \bar{D}_{L}\right)=O_{L}
$$

and $\operatorname{lct}\left(X, \bar{D}_{L}\right)=1 / 2$. Thus, we see that $\operatorname{lct}(X) \leqslant 1 / 2$.

Suppose that $\operatorname{lct}(X)<1 / 2$. Then there exists an effective $\mathbb{Q}$-divisor

$$
D \sim_{\mathbb{Q}}-K_{X}
$$

such that the log pair $(X, \lambda D)$ is not $\log$ canonical for some positive rational number $\lambda<1 / 2$. 
By Remark 2.23, we may assume that $R \not \subset \operatorname{Supp}(D)$, because $\operatorname{lct}(X, R)=5 / 6$.

Let $C$ be a line in $X$ such that $C \not \subset \operatorname{Supp}(D)$. Then

$$
1=D \cdot C \geqslant \operatorname{mult}_{O_{C}}(D) \operatorname{mult}_{O_{C}}(C)=\operatorname{mult}_{O_{C}}(D),
$$

which implies that $O_{C} \notin \operatorname{LCS}(X, \lambda D)$. In particular, we see that $\Sigma_{1} \notin \operatorname{LCS}(X, \lambda D)$.

Let $\Gamma$ be an irreducible curve in $\operatorname{Supp}(D)$ such that $O_{C} \in \Gamma$. Then

$$
\operatorname{mult}_{\Gamma}\left(\frac{1}{2} \bar{D}_{C}+\lambda D\right)=\frac{\operatorname{mult}_{\Gamma}\left(\bar{D}_{C}\right)}{2}+\lambda \operatorname{mult}_{\Gamma}(D) \leqslant \frac{\operatorname{mult}_{\Gamma}\left(\bar{D}_{C}\right)}{2}+\lambda \operatorname{mult}_{O_{C}}(D)<1,
$$

because $\lambda<1 / 2$ and $\operatorname{Sing}\left(\bar{D}_{C}\right)=C$, because $\bar{D}_{C} \neq R$. Thus, we see that

$$
\Gamma \nsubseteq \operatorname{LCS}\left(X, \frac{1}{2} \bar{D}_{C}+\lambda D\right) \supseteq \operatorname{LCS}(X, \lambda D) \cup O_{C},
$$

which is impossible by Theorem 2.7, because $O_{C} \notin \operatorname{LCS}(X, \lambda D)$ and $\lambda<1 / 2$.

The threefold satisfying all hypotheses of Lemma 3.1 is called the Mukai-Mumemura threefold. Remark 3.2. Let $X$ be the Mukai-Mumemura threefold. Then it follows from [55] that

$$
\operatorname{lct}(X, \operatorname{SO}(3))=\frac{5}{6} \text {. }
$$

Remark 3.3. Let $X$ be a smooth Fano threefold such that $\operatorname{Pic}(X)=\mathbb{Z}\left[-K_{X}\right]$. Then it follows from the papers [143], [91], [74] that the following conditions are equivalent:

- $-K_{X}^{3}=22$ and the threefold $X$ is the Mukai-Mumemura threefold;

- $-K_{X}^{3} \geqslant 16$ and for any curve $\mathbb{P}^{1} \cong L \subset X$ such that $-K_{X} \cdot L=1$, we have

$$
\mathcal{N}_{L / X} \cong \mathcal{O}_{\mathbb{P}^{1}}(1) \oplus \mathcal{O}_{\mathbb{P}^{1}}(-2),
$$

- $-K_{X}^{3} \geqslant 6$ and for any two curves $L_{1} \subset X \supset L_{2}$ such that

$$
-K_{X} \cdot L_{1}=-K_{X} \cdot L_{2}=1,
$$

either $L_{1}=L_{2}$, or $L_{1} \cap L_{2}=\varnothing$.

Remark 3.4. Let $Z \subset \mathbb{P}^{2}$ be a plane quartic curve. Then $Z$ is given by an equation

$$
\zeta(x, y, z)=0 \subset \mathbb{P}^{2} \cong \operatorname{Proj}(\mathbb{C}[x, y, z]),
$$

where $\zeta(x, y, z)$ is a form of degree 4 . A polar hexagon of the curve $Z \subset \mathbb{P}^{2}$ is the union

$$
\Gamma=\bigcup_{i=1}^{6}\left(\xi_{i}(x, y, z)=0\right) \subset \mathbb{P}^{2}
$$

such that $\zeta(x, y, z)=\sum_{i=1}^{6} \xi_{i}^{4}(x, y, z)$, where $\xi_{i}(x, y, z)$ is a non-zero linear form. Put

$$
X_{\zeta}=\overline{\left\{\Gamma \in \operatorname{Hilb}_{6}\left(\mathbb{P}^{2}\right) \mid \Gamma \text { is polar to } Z \subset \mathbb{P}^{2}\right\}},
$$

where we identify the polar hexagon $\Gamma$ with a point in $\operatorname{Hilb}_{6}\left(\mathbb{P}^{2}\right)$. Then it follows from [167 that the variety $X_{\zeta}$ is a smooth Fano threefold such that

$$
\operatorname{Pic}\left(X_{\zeta}\right)=\mathbb{Z}\left[-K_{X_{\zeta}}\right]
$$

and the equality $-K_{X_{\zeta}}^{3}=22$ holds in the case when the homogeneous form $\zeta(x, y, z)$ is sufficiently genera 5 . It follows from [130] that $X_{\zeta}$ is the Mukai-Mumemura threefold if

$$
\zeta(x, y, z)=\left(x^{2}+y^{2}+z^{2}\right)^{2}
$$

\footnotetext{
${ }^{5}$ Varieties $X_{\zeta}$ that are smooth compactifications of $\mathbb{C}^{3}$ were studied in [138, 139], 63], 144], 64], 65].
} 


\section{Cubic surfaces}

Let $X$ be a cubic surface in $\mathbb{P}^{3}$ that has at most one ordinary double point.

Definition 4.1. A point $O \in X$ is said to be an Eckardt point if $O \notin \operatorname{Sing}(X)$ and

$$
O=L_{1} \cap L_{2} \cap L_{3}
$$

where $L_{1}, L_{2}, L_{3}$ are different lines on the surface $X \subset \mathbb{P}^{3}$.

General cubic surfaces have no Eckardt points. It follows from Example 1.18 and 1.19 that

$$
\operatorname{lct}(X)=\left\{\begin{array}{l}
3 / 4 \text { when } X \text { has no Eckardt points and } \operatorname{Sing}(X)=\varnothing \\
2 / 3 \text { when } X \text { has an Eckardt point or } \operatorname{Sing}(X) \neq \varnothing
\end{array}\right.
$$

Let $D$ be an effective $\mathbb{Q}$-divisor on $X$ such that $D \sim_{\mathbb{Q}}-K_{X}$, and let $\omega$ be a positive rational number such that $\omega<3 / 4$. In this section we prove the following result (cf. [20], [33], 31]).

Theorem 4.2. Suppose that $(X, \omega D)$ is not log canonical. Then

$$
\operatorname{LCS}(X, \omega D)=O,
$$

where $O \in X$ is either a singular point or an Eckardt point.

Suppose that $(X, \omega D)$ is not $\log$ canonical. Let $P$ be a point in $\operatorname{LCS}(X, \omega D)$. Suppose that

- neither $P=\operatorname{Sing}(X)$,

- nor $P$ is an Eckardt point.

Lemma 4.3. One has $\operatorname{LCS}(X, \omega D)=P$.

Proof. Suppose that $\operatorname{LCS}(X, \omega D) \neq P$. Then there is a curve $C \subset X$ such that

$$
P \in C \subseteq \operatorname{LCS}(X, \omega D)
$$

by Theorem 2.7. Then there is an effective $\mathbb{Q}$-divisor $\Omega$ on $X$ such that $C \not \subset \operatorname{Supp}(\Omega)$ and

$$
D=\mu C+\Omega,
$$

where $\mu \geqslant 1 / \omega$. Let $H$ be a general hyperplane section of $X$. Then

$$
3=H \cdot D=\mu H \cdot C+H \cdot \Omega \geqslant \mu \operatorname{deg}(C),
$$

which implies that either $\operatorname{deg}(C)=1$, or $\operatorname{deg}(C)=2$.

Suppose that $\operatorname{deg}(C)=1$. Let $Z$ be a general conic on $X$ such that $-K_{X} \sim C+Z$. Then

$$
2=Z \cdot D=\mu Z \cdot C+Z \cdot \Omega \geqslant \mu Z \cdot C=\left\{\begin{array}{l}
2 \mu \text { if } C \cap \operatorname{Sing}(X)=\varnothing \\
3 \mu / 2 \text { if } C \cap \operatorname{Sing}(X) \neq \varnothing,
\end{array}\right.
$$

which implies that $\mu \leqslant 4 / 3$. But $\mu \geqslant 1 / \omega>4 / 3$, which gives a contradiction.

We see that $\operatorname{deg}(C)=2$. Let $L$ be a line on $X$ such that $-K_{X} \sim C+L$. Then

$$
D=\mu C+\lambda L+\Upsilon,
$$

where $\lambda \in \mathbb{Q}$ such that $\lambda \geqslant 0$, and $\Upsilon$ is an effective $\mathbb{Q}$-divisor such that $C \not \subset \operatorname{Supp}(\Upsilon) \not \supset L$. Then

$$
1=L \cdot D=\mu L \cdot C+\lambda L \cdot L+L \cdot \Upsilon \geqslant \mu L \cdot C+\lambda L \cdot L=\left\{\begin{array}{l}
2 \mu-\lambda \text { if } C \cap \operatorname{Sing}(X)=\varnothing \\
3 \mu / 2-\lambda / 2 \text { if } C \cap \operatorname{Sing}(X) \neq \varnothing,
\end{array}\right.
$$

which implies that $\mu \leqslant 7 / 6<4 / 3$, because $\lambda \leqslant 4 / 3$ (see the case when $\operatorname{deg}(C)=1$ ). But $\mu>4 / 3$, which gives a contradiction.

Let $\pi: U \rightarrow X$ be a blow up of $P$, and let $E$ be the $\pi$-exceptional curve. Then

$$
\bar{D} \sim_{\mathbb{Q}} \pi^{*}(D)+\operatorname{mult}_{P}(D) E,
$$

where $\operatorname{mult}_{P}(D) \geqslant 1 / \omega$ and $\bar{D}$ is a proper transform of $D$ on the surface $U$. The log pair

$$
\left(U, \omega \bar{D}+\left(\omega \operatorname{mult}_{P}(D)-1\right) E\right)
$$


is not $\log$ canonical at some point $Q \in E$. Then either $\operatorname{mult}_{P}(D) \geqslant 2 / \omega$, or

$$
\operatorname{mult}_{Q}(\bar{D})+\operatorname{mult}_{P}(D) \geqslant 2 / \omega>8 / 3,
$$

because the divisor $\omega \bar{D}+\left(\omega \operatorname{mult}_{P}(D)-1\right) E$ is effective.

Let $T$ be the unique hyperplane section of $X$ that is singular at $P$. We may assume that

$$
\operatorname{Supp}(T) \nsubseteq \operatorname{Supp}(D)
$$

by Remark 2.23 , because $(X, \omega T)$ is $\log$ canonical. The following cases are possible:

- the curve $T$ is irreducible;

- the curve $T$ is a union of a line and an irreducible conic;

- the curve $T$ consists of 3 lines.

Hence $T$ is reduced. Note that $\operatorname{mult}_{P}(T)=2$ since $P$ is not an Eckardt point. We exclude these cases one by one.

Lemma 4.5. The curve $T$ is reducible.

Proof. Suppose that $T$ is irreducible. Then there is a commutative diagram

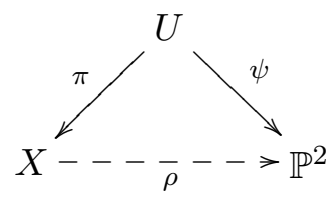

where $\psi$ is a double cover branched over a quartic curve, and $\rho$ is the projection from $P \in X$.

Let $\bar{T}$ be the proper transform of $T$ on the surface $U$. Suppose that $Q \in \bar{T}$. Then

$3-2 \operatorname{mult}_{P}(D)=\bar{T} \cdot \bar{D} \geqslant \operatorname{mult}_{Q}(\bar{T}) \operatorname{mult}_{Q}(\bar{D})>\operatorname{mult}_{Q}(\bar{T})\left(8 / 3-\operatorname{mult}_{P}(D)\right) \geqslant 8 / 3-\operatorname{mult}_{P}(D)$, which implies that $\operatorname{mult}_{P}(D) \leqslant 1 / 3$. But $\operatorname{mult}_{P}(D)>4 / 3$. Thus, we see that $Q \notin \bar{T}$.

Let $\tau \in \operatorname{Aut}(U)$ be an involution 6 induced by $\psi$. It follows from [118, that

$$
\tau^{*}\left(\pi^{*}\left(-K_{X}\right)\right) \sim \pi^{*}\left(-2 K_{X}\right)-3 E,
$$

and $\tau(\bar{T})=E$. Put $\breve{Q}=\pi \circ \tau(Q)$. Then $\breve{Q} \neq P$, because $Q \notin \bar{T}$.

Let $H$ be the hyperplane section of $X$ that is singular at $\breve{Q}$. Then $T \neq H$, because $P \neq \breve{Q}$ and $T$ is smooth outside of the point $P$. Hence $P \notin H$, because otherwise

$$
3=H \cdot T \geqslant \operatorname{mult}_{P}(H) \operatorname{mult}_{P}(T)+\operatorname{mult}_{\breve{Q}}(H) \operatorname{mult}_{\breve{Q}}(T) \geqslant 4 \text {. }
$$

Let $\bar{H}$ be the proper transform of $H$ on the surface $U$. Put $\bar{R}=\tau(\bar{H})$ and $R=\pi(\bar{R})$. Then

$$
\bar{R} \sim \pi^{*}\left(-2 K_{X}\right)-3 E
$$

ant the curve $\bar{R}$ must be singular at the point $Q$.

Suppose that $R$ is irreducible. Taking into account the possible singularities of $\bar{R}$, we see that

$$
\left(X, \frac{3}{8} R\right)
$$

is $\log$ canonical. Thus, we may assume that $R \nsubseteq \operatorname{Supp}(D)$ by Remark 2.23. Then

$$
6-3 \operatorname{mult}_{P}(D)=\bar{R} \cdot \bar{D} \geqslant \operatorname{mult}_{Q}(\bar{R}) \operatorname{mult}_{Q}(\bar{D})>2\left(8 / 3-\operatorname{mult}_{P}(D)\right),
$$

which implies that $\operatorname{mult}_{P}(D)<2 / 3$. But $\operatorname{mult}_{P}(D)>4 / 3$. The curve $R$ must be reducible.

The curves $R$ and $H$ are reducible. So, there is a line $L \subset X$ such that $P \notin L \ni \breve{Q}$.

Let $\bar{L}$ be the proper transform of $L$ on the surface $U$. Put $\bar{Z}=\tau(\bar{L})$. Then $\bar{L} \cdot E=0$ and

$$
\bar{L} \cdot \bar{T}=\bar{L} \cdot \pi^{*}\left(-K_{X}\right)=1 \text {, }
$$

which implies that $\bar{Z} \cdot E=1$ and $\bar{Z} \cdot \pi^{*}\left(-K_{X}\right)=2$. We have $Q \in \bar{Z}$. Then

$$
2-\operatorname{mult}_{P}(D)=\bar{Z} \cdot \bar{D} \geqslant \operatorname{mult}_{Q}(\bar{D})>8 / 3-\operatorname{mult}_{P}(D)>2-\operatorname{mult}_{P}(D)
$$

in the case when $\bar{Z} \nsubseteq \operatorname{Supp}(\bar{D})$. Hence, we see that $\bar{Z} \subseteq \operatorname{Supp}(\bar{D})$.

\footnotetext{
${ }^{6}$ The involution $\tau$ induces an involution in $\operatorname{Bir}(X)$ that is called a Geiser involution.
} 
Put $Z=\pi(\bar{Z})$. Then $Z$ is a conic such that $P \in Z$ and

$$
-K_{X} \sim L+Z
$$

which means that $L \cup Z$ is cut out by the plane in $\mathbb{P}^{3}$ that passes through $Z$. Put

$$
D=\varepsilon Z+\Upsilon
$$

where $\varepsilon \in \mathbb{Q}$ such that $\varepsilon \geqslant 0$, and $\Upsilon$ is an effective $\mathbb{Q}$-divisor such that $Z \not \subset \operatorname{Supp}(\Upsilon)$.

We may assume that $L \nsubseteq \operatorname{Supp}(\Upsilon)$ by Remark 2.23 . Then

$$
1=L \cdot D=\varepsilon Z \cdot L+L \cdot \Upsilon \geqslant \varepsilon Z \cdot L=\left\{\begin{array}{l}
2 \varepsilon \text { if } Z \cap \operatorname{Sing}(X)=\varnothing, \\
3 \varepsilon / 2 \text { if } Z \cap \operatorname{Sing}(X) \neq \varnothing,
\end{array}\right.
$$

which implies that $\varepsilon \leqslant 2 / 3$.

Let $\bar{\Upsilon}$ be the proper transform of $\Upsilon$ on the surface $U$. Then the log pair

$$
\left(U, \varepsilon \omega \bar{Z}+\omega \bar{\Upsilon}+\left(\omega \operatorname{mult}_{P}(D)-1\right) E\right)
$$

is not $\log$ canonical at $Q \in \bar{Z}$. Then

$$
\omega \bar{\Upsilon} \cdot \bar{Z}+\left(\operatorname{wmult}_{P}(D)-1\right)=\left(\omega \bar{\Upsilon}+\left(\operatorname{wmult}_{P}(D)-1\right) E\right) \cdot \bar{Z}>1
$$

by Lemma 2.21 , because $\varepsilon \leqslant 2 / 3$. In particular, we see that

$8 / 3-\operatorname{mult}_{P}(D)<\bar{Z} \cdot \bar{\Upsilon}=2-\operatorname{mult}_{P}(D)-\varepsilon \bar{Z} \cdot \bar{Z}=\left\{\begin{array}{l}2-\operatorname{mult}_{P}(D)+\varepsilon \text { if } Z \cap \operatorname{Sing}(X)=\varnothing, \\ 2-\operatorname{mult}_{P}(D)+\varepsilon / 2 \text { if } Z \cap \operatorname{Sing}(X) \neq \varnothing,\end{array}\right.$

which implies that $\varepsilon>2 / 3$. But $\varepsilon \leqslant 2 / 3$.

Therefore, there is a line $L_{1} \subset X$ such that $P \in L_{1}$. Put

$$
D=m_{1} L_{1}+\Omega
$$

where $m_{1} \in \mathbb{Q}$ such that $\Omega$ is an effective $\mathbb{Q}$-divisor such that $L_{1} \not \subset \in \operatorname{Supp}(\Omega)$. Then

$$
4 / 3<1 / \omega<\Omega \cdot L_{1}=1-m_{1} L_{1} \cdot L_{1}=\left\{\begin{array}{l}
1+m_{1} \text { if } L_{1} \cap \operatorname{Sing}(X)=\varnothing, \\
1+m_{1} / 2 \text { if } L_{1} \cap \operatorname{Sing}(X) \neq \varnothing .
\end{array}\right.
$$

Corollary 4.6. The following inequality holds:

$$
m_{1}>\left\{\begin{array}{l}
1 / 3 \text { if } L_{1} \cap \operatorname{Sing}(X)=\varnothing \\
2 / 3 \text { if } L_{1} \cap \operatorname{Sing}(X) \neq \varnothing
\end{array}\right.
$$

Remark 4.7. Suppose that $X$ is singular. Put $O=\operatorname{Sing}(X)$. It follows from [16] that

$$
O=\Gamma_{1} \cap \Gamma_{2} \cap \Gamma_{3} \cap \Gamma_{4} \cap \Gamma_{5} \cap \Gamma_{6},
$$

where $\Gamma_{1}, \ldots, \Gamma_{6}$ are different lines on the surface $X \subset \mathbb{P}^{3}$. The equivalence

$$
-2 K_{X} \sim \sum_{i=1}^{6} \Gamma_{i}
$$

holds. Suppose that $L_{1}=\Gamma_{1}$. Let $\Pi_{2}, \ldots, \Pi_{6} \subset \mathbb{P}^{3}$ be planes such that

$$
L_{1} \subset \Pi_{i} \supset \Gamma_{i},
$$

and let $\Lambda_{2}, \ldots, \Lambda_{6}$ be lines on the surface $X$ such that

$$
L_{1} \cup \Gamma_{i} \cup \Lambda_{i}=\Pi_{i} \cap X \subset X \subset \mathbb{P}^{3},
$$

which implies that $-K_{X} \sim L_{1}+\Gamma_{i}+\Lambda_{i}$. Then

$$
-5 K_{X} \sim 4 L_{1}+\sum_{i=2}^{6} \Lambda_{i}+\left(L_{1}+\sum_{\substack{i=2 \\ 36}}^{6} \Gamma_{i}\right) \sim 4 L_{1}+\sum_{i=2}^{6} \Lambda_{i}-2 K_{X},
$$


which implies that $-3 K_{X} \sim 4 L_{1}+\sum_{i=2}^{6} \Lambda_{i}$. But the log pair

$$
\left(X, L_{1}+\frac{\sum_{i=2}^{6} \Lambda_{i}}{3}\right)
$$

is $\log$ canonical at the point $P$. Thus, we may assume that

$$
\operatorname{Supp}\left(\sum_{i=2}^{6} \Lambda_{i}\right) \nsubseteq \operatorname{Supp}(D)
$$

thanks to Remark 2.23, because $L_{1} \subseteq \operatorname{Supp}(D)$. Then there is $\Lambda_{k}$ such that

$$
1=D \cdot \Lambda_{k}=\left(m_{1} L_{1}+\Omega\right) \cdot \Lambda_{k}=m_{1}+\Omega \cdot \Lambda_{k} \geqslant m_{1}
$$

because $O \notin \Lambda_{k}$. Thus, we may assume that $m_{1} \leqslant 1$ if $L_{1} \cap \operatorname{Sing}(X) \neq \varnothing$.

Arguing as in the proof of Lemma 2.15, we see that $m_{1} \leqslant 1$ if $L_{1} \cap \operatorname{Sing}(X)=\varnothing$.

Lemma 4.8. There is a line $L_{2} \subset X$ such that $L_{1} \neq L_{2}$ and $P \in L_{2}$.

Proof. Suppose that there is no line $L_{2} \subset X$ such that $L_{1} \neq L_{2}$ and $P \in L_{2}$. Then

$$
T=L_{1}+C,
$$

where $C$ is an irreducible conic on the surface $X \subset \mathbb{P}^{3}$ such that $P \in C$.

It follows from Remark 2.23 that we may assume that $C \nsubseteq \operatorname{Supp}(\Omega)$, because $m_{1} \neq 0$.

Let $\bar{L}_{1}$ and $\bar{C}$ be the proper transforms of $L_{1}$ and $C$ on the surface $U$, respectively. Then

$$
\bar{D} \sim_{\mathbb{Q}} m_{1} \bar{L}_{1}+\bar{\Omega} \sim_{\mathbb{Q}} \pi^{*}\left(m_{1} L_{1}+\Omega\right)-\left(m_{1}+\operatorname{mult}_{P}(\Omega)\right) E \sim_{\mathbb{Q}} \pi^{*}(D)-\operatorname{mult}_{P}(D) E,
$$

where $\bar{\Omega}$ is the proper transform of the divisor $\Omega$ on the surface $U$. We have

$0 \leqslant \bar{C} \cdot \bar{\Omega}=2-\operatorname{mult}_{P}(D)+m_{1} \bar{C} \cdot \bar{L}<2 / 3-m_{1} \bar{C} \cdot \bar{L}_{1}=\left\{\begin{array}{l}2 / 3-m_{1}, \text { if } L_{1} \cap \operatorname{Sing}(X)=\varnothing, \\ 2 / 3-m_{1} / 2, \text { if } L_{1} \cap \operatorname{Sing}(X) \neq \varnothing,\end{array}\right.$

which implies that $m_{1}<2 / 3$ if $L_{1} \cap \operatorname{Sing}(X)=\varnothing$. It follows from inequality 4.4 that

$$
\operatorname{mult}_{Q}(\bar{\Omega})>8 / 3-\operatorname{mult}_{P}(\Omega)-m_{1}\left(1+\operatorname{mult}_{Q}\left(\bar{L}_{1}\right)\right) .
$$

Suppose that $Q \in \bar{L}_{1}$. Then it follows from Lemma 2.21 that

$$
8 / 3<\bar{L}_{1} \cdot\left(\bar{\Omega}+\left(\operatorname{mult}_{P}(\Omega)+m_{1}\right) E\right)=1-m_{1} \bar{L}_{1} \cdot \bar{L}_{1}=\left\{\begin{array}{l}
1+2 m_{1}, \text { if } L_{1} \cap \operatorname{Sing}(X)=\varnothing, \\
1+3 m_{1} / 2, \text { if } L_{1} \cap \operatorname{Sing}(X) \neq \varnothing,
\end{array}\right.
$$

which is impossible, because $m_{1} \leqslant 1$ if $L_{1} \cap \operatorname{Sing}(X) \neq \varnothing$ by Remark 4.7.

We see that $Q \notin \bar{L}_{1}$. Suppose that $Q \in \bar{C}$. Then

$$
2-\operatorname{mult}_{P}(\Omega)-m_{1}-m_{1} \bar{C} \cdot \bar{L} 1=\bar{C} \cdot \bar{\Omega}>8 / 3-\operatorname{mult}_{P}(\Omega)-m_{1}
$$

which is impossible, because $m_{1} \bar{C} \cdot \bar{L}_{1} \geqslant 0$. Hence, we see that $Q \notin \bar{C}$.

There is a commutative diagram

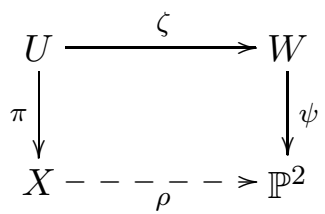

where $\zeta$ is a birational morphism that contracts the curve $\bar{L}_{1}$, the morphism $\psi$ is a double cover branched over a quartic curve, and $\rho$ is a linear projection from the point $P \in X$.

Let $\tau$ be the birational involution of $U$ induced by $\psi$. Then

- the involution $\tau$ is biregular $\Longleftrightarrow L_{1} \cap \operatorname{Sing}(X)=\varnothing$,

- the involution $\tau$ acts biregularly on $U \backslash \bar{L}_{1}$ if $L_{1} \cap \operatorname{Sing}(X) \neq \varnothing$,

- it follows from the construction of $\tau$ that $\tau(E)=\bar{C}$, 
- if $L_{1} \cap \operatorname{Sing}(X)=\varnothing$, then

$$
\tau^{*}\left(\bar{L}_{1}\right) \sim \bar{L}_{1}, \tau^{*}(E) \sim \bar{C}, \tau^{*}\left(\pi^{*}\left(-K_{X}\right)\right) \sim \pi^{*}\left(-2 K_{X}\right)-3 E-\bar{L}_{1} .
$$

Let $H$ be the hyperplane section of $X$ that is singular at $\pi \circ \tau(Q) \in C$. Then $P \notin H$, because $C$ is smooth. Let $\bar{H}$ be the proper transform of $H$ on the surface $U$. Then

$$
\bar{L}_{1} \not \subseteq \operatorname{Supp}(\bar{H}) \nsupseteq \bar{C},
$$

and we can put $\bar{R}=\tau(\bar{H})$ and $R=\pi(\bar{R})$. Then $\bar{R}$ is singular at the point $Q$, and

$$
\bar{R} \sim \pi^{*}\left(-2 K_{X}\right)-3 E-\bar{L}_{1},
$$

because $R$ does not pass through a singular point of $X$ if $\operatorname{Sing}(X) \neq \varnothing$.

Suppose that $R$ is irreducible. Then $R+L_{1} \sim-2 K_{X}$, but the log pair

$$
\left(X, \frac{3}{8}\left(R+L_{1}\right)\right)
$$

is $\log$ canonical. Thus, we may assume that $R \nsubseteq \operatorname{Supp}(D)$ by Remark 2.23 . Then

$5-2\left(m_{1}+\operatorname{mult}_{P}(\Omega)\right)+m_{1}\left(1+\bar{L}_{1} \cdot \bar{L}_{1}\right)=\bar{R} \cdot \bar{\Omega} \geqslant 2 \operatorname{mult}_{Q}(\bar{\Omega})>2\left(8 / 3-m_{1}-\operatorname{mult}_{P}(\Omega)\right)$, which implies that $m_{1}<0$. The curve $R$ must be reducible.

There is a line $L \subset X$ such that $P \notin L$ and $\pi \circ \tau(Q) \in L$. Then

$$
L \cap L_{1}=\varnothing \text {, }
$$

because $\pi \circ \tau(Q) \in C$ and $\left(C+L_{1}\right) \cdot L=T \cdot L=1$. Thus, there is unique conic $Z \subset X$ such that $-K_{X} \sim L+Z$ and $P \in Z$. Then $Z$ is irreducible and $P=Z \cap L_{1}$, because $(L+Z) \cdot L_{1}=1$.

Let $\bar{L}$ and $\bar{Z}$ be the proper transform of $L$ and $Z$ on the surface $U$, respectively. Then

$\bar{L} \cdot \bar{C}=\bar{Z} \cdot E=1, \bar{L}_{1} \cdot \bar{Z}=\bar{L} \cdot E=\bar{L} \cdot \bar{L}_{1}=0, \bar{Z} \cdot \bar{Z}=1-\bar{L} \cdot \bar{Z}, \bar{L} \cdot \bar{Z}=\left\{\begin{array}{l}2 \text { if } L \cap \operatorname{Sing}(X)=\varnothing, \\ 3 / 2 \text { if } L \cap \operatorname{Sing}(X) \neq \varnothing .\end{array}\right.$

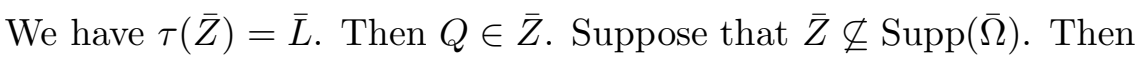

$$
2-m_{1}-\operatorname{mult}_{P}(\Omega)=\bar{Z} \cdot \bar{\Omega}>8 / 3-m_{1}-\operatorname{mult}_{P}(\Omega),
$$

which is impossible. Thus, we see that $\bar{Z} \subseteq \operatorname{Supp}(\bar{\Omega})$. But the log pair

$$
(X, \omega(L+Z))
$$

is $\log$ canonical at the point $P$. Hence, we may assume that $\bar{L} \nsubseteq \operatorname{Supp}(\bar{\Omega})$ by $\operatorname{Remark} 2.23$. Put

$$
D=\varepsilon Z+m_{1} L_{1}+\Upsilon,
$$

where $\Upsilon$ is an effective $\mathbb{Q}$-divisor such that $Z \not \subset \operatorname{Supp}(\Upsilon) \not \supset L_{1}$. Then

$$
1=L \cdot D=\varepsilon L \cdot Z+m_{1} L \cdot L_{1}+L \cdot \Upsilon=\varepsilon L \cdot Z+L \cdot \Upsilon \geqslant \varepsilon L \cdot Z=\left\{\begin{array}{l}
2 \varepsilon \text { if } L \cap \operatorname{Sing}(X)=\varnothing, \\
3 \varepsilon / 2 \text { if } L \cap \operatorname{Sing}(X) \neq \varnothing,
\end{array}\right.
$$

which implies that $\varepsilon \leqslant 2 / 3$. But $\bar{Z} \cap \bar{L}_{1}=\varnothing$. Then it follows from Lemma 2.21 that

$$
2-\operatorname{mult}_{P}(D)-\varepsilon \bar{Z} \cdot \bar{Z}=\bar{Z} \cdot \bar{\Upsilon}>8 / 3-\operatorname{mult}_{P}(D),
$$

where $\bar{\Upsilon}$ is a proper transform of $\Upsilon$ on the surface $U$. We deduce that $\varepsilon>2 / 3$. But $\varepsilon \leqslant 2 / 3$.

Therefore, we see that $T=L_{1}+L_{2}+L_{3}$, where $L_{3}$ is a line such that $P \notin L_{3}$. Put

$$
D=m_{1} L_{1}+m_{2} L_{2}+\Delta,
$$

where $\Delta$ is an effective $\mathbb{Q}$-divisor such that $L_{2} \not \subset \operatorname{Supp}(\Delta) \nsupseteq L_{2}$.

The inequalities $m_{1}>1 / 3$ and $m_{2}>1 / 3$ hold by Corollary 4.6. We may assume that $L_{3} \not \subset \operatorname{Supp}(\Delta)$ by Remark 2.23. If the singular point of $X$ (provided that there exists one) is contained in either $L_{1}$ or $L_{2}$, we may assume without loss of generality that it is contained in $L_{1}$. Then $L_{3} \cdot L_{2}=1$ and $L_{3} \cdot L_{1}=1 / 2$ in the case when $L_{1} \cap \operatorname{Sing}(X) \neq \varnothing$, and

$$
L_{3} \cdot L_{2}=L_{3} \cdot L_{1}=1
$$

in the case when $L_{1} \cap \operatorname{Sing}(X)=\varnothing$. Then $1-m_{1} L_{1} \cdot L_{3}-m_{2}=L_{3} \cdot \Delta \geqslant 0$. 
Let $\bar{L}_{1}$ and $\bar{L}_{3}$ be the proper transforms of $L_{1}$ and $L_{2}$ on the surface $U$, respectively. Then

$$
m_{1} \bar{L}_{1}+m_{2} \bar{L}_{2}+\bar{\Delta} \sim_{\mathbb{Q}} \pi^{*}\left(m_{1} L_{1}+m_{2} L_{2}+\Delta\right)-\left(m_{1}+m_{2}+\operatorname{mult}_{P}(\Delta)\right) E,
$$

where $\bar{\Delta}$ is the proper transform of $\Delta$ on the surface $U$. The inequality 4.4 implies that

$$
\operatorname{mult}_{Q}(\bar{\Delta})>8 / 3-\operatorname{mult}_{P}(\Delta)-m_{1}\left(1+\operatorname{mult}_{Q}\left(\bar{L}_{1}\right)\right)-m_{1}\left(1+\operatorname{mult}_{Q}\left(\bar{L}_{2}\right)\right) .
$$

Lemma 4.10. The curve $\bar{L}_{2}$ does not contain the point $Q$.

Proof. Suppose that $Q \in \bar{L}_{2}$. Then

$$
1-\operatorname{mult}_{P}(\Delta)-m_{1}+m_{2}=\bar{L}_{2} \cdot \bar{\Delta}>8 / 3-\operatorname{mult}_{P}(\Delta)-m_{1}-m_{2}
$$

by Lemma 2.21, Thus, we have $m_{2}>5 / 6$. It follows from Lemma 2.21 that

$$
1-m_{2}-m_{1} L_{1} \cdot L_{1}=\Delta \cdot L_{1}>4 / 3-m_{2},
$$

but $L_{1} \cdot L_{1}=-1$ if $L_{1} \cap \operatorname{Sing}(X)=\varnothing$, and $L_{1} \cdot L_{1}=-1 / 2$ if $L_{1} \cap \operatorname{Sing}(X) \neq \varnothing$. Then

$$
m_{1}>\left\{\begin{array}{l}
1 / 3 \text { if } L_{1} \cap \operatorname{Sing}(X)=\varnothing, \\
2 / 3 \text { if } L_{1} \cap \operatorname{Sing}(X) \neq \varnothing,
\end{array}\right.
$$

by Corollary 4.6, which is impossible because $m_{2}>5 / 6$ and

$$
1>m_{1} L_{1} \cdot L_{3}+m_{2} .
$$

Lemma 4.11. The curve $\bar{L}_{1}$ does not contain the point $Q$.

Proof. Suppose that $Q \in \bar{L}_{1}$. Arguing as in the proof of Lemma 4.10, we see that

$$
L_{1} \cap \operatorname{Sing}(X) \neq \varnothing,
$$

which implies that $\bar{L}_{1} \cdot \bar{L}_{1}=-1 / 2$. Then $m_{1}>10 / 9$, because

$$
1+3 m_{1} / 2=\bar{L}_{2} \cdot\left(\bar{\Delta}+\left(\operatorname{mult}_{P}(\Delta)-m_{1}-m_{2}\right) E\right)>8 / 3
$$

by Lemma 2.21, But $m_{1} \leqslant 1$ by Remark 4.7 .

Therefore, we see that $\bar{L}_{1} \not \ngtr Q \notin \bar{L}_{2}$. There is a commutative diagram

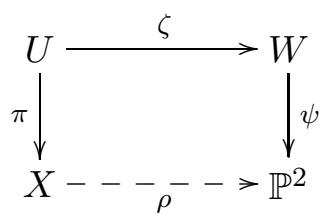

where $\zeta$ is a birational morphism that contracts the curves $\bar{L}_{1}$ and $\bar{L}_{2}$, the morphism $\psi$ is a double cover branched over a quartic curve, and $\rho$ is the projection from the point $P$.

Let $\tau$ be the birational involution of $U$ induced by $\psi$. Then

- the involution $\tau$ is biregular $\Longleftrightarrow L_{1} \cap \operatorname{Sing}(X)=\varnothing$,

- the involution $\tau$ acts biregularly on $U \backslash \bar{L}_{1}$ if $L_{1} \cap \operatorname{Sing}(X) \neq \varnothing$,

- the equality $\tau\left(\bar{L}_{2}\right)=\bar{L}_{2}$ holds,

- if $L_{1} \cap \operatorname{Sing}(X)=\varnothing$, then $\tau\left(\bar{L}_{1}\right)=\bar{L}_{1}$ and

$$
\tau^{*}\left(\pi^{*}\left(-K_{X}\right)\right) \sim \pi^{*}\left(-2 K_{X}\right)-3 E-\bar{L}_{1}-\bar{L}_{2} .
$$

Let $\bar{L}_{3}$ be a proper transform of $L_{3}$ on the surface $U$. Then $\tau(E)=\bar{L}_{3}$ and

$$
L_{1} \cup L_{2} \not \supset \pi \circ \tau(Q) \in L_{3} .
$$

Lemma 4.12. The line $L_{3}$ is the only line on $X$ that passes through the point $\pi \circ \tau(Q)$. 
Proof. Suppose that there is a line $L \subset X$ such that $L \neq L_{3}$ and $\pi \circ \tau(Q) \in L$. Then

$$
L \cap L_{1}=L \cap L_{2}=\varnothing,
$$

because $\pi \circ \tau(Q) \in L_{3}$ and $\left(L_{1}+L_{2}+L_{3}\right) \cdot L=1$. Thus, there is unique conic $Z \subset X$ such that $-K_{X} \sim L+Z$ and $P \in Z$. Then $Z$ is irreducible, because $P \notin L$ and $P$ is not an Eckardt point.

Let $\bar{L}$ and $\bar{Z}$ be the proper transform of $L$ and $Z$ on the surface $U$, respectively. Then

$$
\bar{L} \cdot \bar{L}_{3}=\bar{Z} \cdot E=1, \bar{Z} \cdot \bar{Z}=1-\bar{L} \cdot \bar{Z}, \bar{L} \cdot \bar{Z}=\left\{\begin{array}{l}
2 \text { if } L \cap \operatorname{Sing}(X)=\varnothing, \\
3 / 2 \text { if } L \cap \operatorname{Sing}(X) \neq \varnothing,
\end{array}\right.
$$

and $\bar{L}_{1} \cdot \bar{Z}=\bar{L}_{2} \cdot \bar{Z}=\bar{L} \cdot E=\bar{L} \cdot \bar{L}_{1}=\bar{L} \cdot \bar{L}_{2}=0$.

We have $\tau(\bar{Z})=\bar{L}$. Then $Q \in \bar{Z}$, which implies that $\bar{Z} \subseteq \operatorname{Supp}(\bar{\Delta})$, because

$$
2-\operatorname{mult}_{P}(\Delta)-m_{1}-m_{2}=\bar{Z} \cdot \bar{\Omega}>8 / 3-\operatorname{mult}_{P}(\Delta)-m_{1}-m_{2}
$$

in the case when $\bar{Z} \nsubseteq \operatorname{Supp}(\bar{\Delta})$. On the other hand, the log pair

$$
(X, \omega(L+Z))
$$

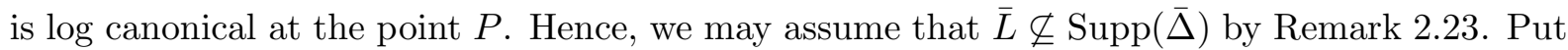

$$
D=\varepsilon Z+m_{1} L_{1}+m_{2} L_{2}+\Upsilon,
$$

where $\Upsilon$ is an effective $\mathbb{Q}$-divisor such that $Z \nsubseteq \operatorname{Supp}(\Upsilon)$. Then

$$
1=L \cdot D=\varepsilon L \cdot Z+m_{1} L \cdot L_{1}+L \cdot \Upsilon=\varepsilon L \cdot Z+L \cdot \Upsilon \geqslant \varepsilon L \cdot Z=\left\{\begin{array}{l}
2 \varepsilon \text { if } L \cap \operatorname{Sing}(X)=\varnothing, \\
3 \varepsilon / 2 \text { if } L \cap \operatorname{Sing}(X) \neq \varnothing,
\end{array}\right.
$$

which implies that $\varepsilon \leqslant 2 / 3$. But $\bar{Z} \cap \bar{L}_{1}=\varnothing$. Then it follows from Lemma 2.21 that

$$
2-\operatorname{mult}_{P}(D)-\varepsilon \bar{Z} \cdot \bar{Z}=\bar{Z} \cdot \bar{\Upsilon}>8 / 3-\operatorname{mult}_{P}(D),
$$

where $\bar{\Upsilon}$ is a proper transform of $\Upsilon$ on the surface $U$. We deduce that $\varepsilon>2 / 3$. But $\varepsilon \leqslant 2 / 3$.

Therefore, there is an unique irreducible conic $C \subset X$ such that

$$
-K_{X} \sim L_{3}+C
$$

and $\pi \circ \tau(Q) \in C$. Then $C+L_{3}$ is a hyperplane section of $X$ that is singular at $\pi \circ \tau(Q)$.

Let $\bar{C}$ be the proper transform of $C$ on the surface $U$. Put $\bar{Z}=\tau(\bar{C})$ and $Z=\pi(\bar{Z})$.

Lemma 4.13. One has $L_{1} \cap \operatorname{Sing}(X) \neq \varnothing$.

Proof. Suppose that $L_{1} \cap \operatorname{Sing}(X)=\varnothing$. Then

$$
C \cap L_{1}=C \cap L_{2}=\varnothing,
$$

because $\left(L_{1}+L_{2}+L_{3}\right) \cdot C=L_{3} \cdot C=2$. One can easily check that

$$
\bar{Z} \sim \pi^{*}\left(-2 K_{X}\right)-4 E-\bar{L}_{1}-\bar{L}_{2}
$$

and $Z$ is singular at $P$. Then $-2 K_{X} \sim Z+L_{1}+L_{2}$. But the log pair

$$
\left(U, \frac{1}{2}\left(Z+L_{1}+L_{2}\right)\right)
$$

is $\log$ canonical at $P$. Thus, we may assume that $Z \nsubseteq \operatorname{Supp}(D)$ by Remark 2.23.

We have $Q \in \bar{Z}$ and $\bar{Z} \cdot E=2$. Then it follows from the inequality 4.4 that

$$
4-2 \operatorname{mult}_{P}(D)=\bar{Z} \cdot \bar{D} \geqslant \operatorname{mult}_{Q}(\bar{D})>8 / 3-\operatorname{mult}_{P}(D),
$$

which implies that $\operatorname{mult}_{P}(D)<4 / 3$. But $\operatorname{mult}_{P}(D)>4 / 3$.

Thus, we see that $L_{1} \cap L_{3}=\operatorname{Sing}(X) \neq \varnothing$. Then $L_{1} \cap L_{2} \in C$, which implies that

$$
\bar{Z} \sim \pi^{*}\left(-2 K_{X}\right)-4 E-2 \bar{L}_{1}-\bar{L}_{2},
$$

and $Z$ is smooth rational cubic curve. Then $-2 K_{X} \sim Z+2 L_{1}+L_{2}$. But the log pair

$$
\left(U, \frac{1}{2}\left(Z+2 L_{1}+L_{2}\right)\right)
$$


is $\log$ canonical at $P$. Thus, we may assume that $Z \nsubseteq \operatorname{Supp}(D)$ by Remark 2.23 .

We have $Q \in \bar{Z}$ and $\bar{Z} \cdot E=\bar{L}_{1}=1$. Then it follows from the inequality 4.4 that

$$
3-\operatorname{mult}_{P}(\Delta)-2 m_{1}-m_{2}=\bar{Z} \cdot \bar{\Delta} \geqslant \operatorname{mult}_{Q}(\bar{\Delta})>8 / 3-\operatorname{mult}_{P}(\Delta)-m_{1}-m_{2},
$$

which implies that $m_{1}<1 / 3$. But $m_{1}>2 / 3$ by Corollary 4.6 .

The obtained contradiction completes the proof Theorem 4.2

\section{Del Pezzo surfaces}

Let $X$ be a del Pezzo surface that has at most canonical singularities, let $O$ be a point of the surface $X$, and let $B_{X}$ be an effective $\mathbb{Q}$-divisor on the surface $X$. Suppose that

- the point $O$ is either smooth or an ordinary double point of $X$,

- the surface $X$ is smooth outside the point $O \in X$.

Lemma 5.1. Suppose that $\operatorname{Sing}(X)=O, K_{X}^{2}=2$ and the equivalence

$$
B_{X} \sim_{\mathbb{Q}}-\mu K_{X}
$$

holds, where $0<\mu<2 / 3$. Then $\mathbb{L} \mathbb{C} \mathbb{S}\left(X, \mu B_{X}\right)=\varnothing$.

Proof. Suppose that $\mathbb{L} \mathbb{C S}\left(X, \mu B_{X}\right) \neq \varnothing$. There is a curve $\mathbb{P}^{1} \cong L \subset X$ such that

$$
\operatorname{LCS}\left(X, \mu B_{X}\right) \nsubseteq L
$$

the equality $L \cdot L=-1$ holds, and $L \cap \operatorname{Sing}(X)=\varnothing$. Thus, there is a birational morphism $\pi: X \rightarrow S$ that contracts the curve $L$. Then

$$
\mathbb{L} \mathbb{C} S\left(S, \mu \pi\left(B_{X}\right)\right) \neq \varnothing
$$

due to the choice of the curve $L \subset X$. But $-K_{S} \sim_{\mathbb{Q}} \pi\left(B_{X}\right)$, and $S \subset \mathbb{P}^{3}$ is a cubic surface that has at most one ordinary double point, which is impossible (see Examples 1.19 and 1.18).

Lemma 5.2. Suppose that $\operatorname{Sing}(X)=\varnothing, K_{X}^{2}=5$, the equivalence

$$
B_{X} \sim_{\mathbb{Q}}-\mu K_{X}
$$

holds, where $\mu \in \mathbb{Q}$ is such that $0<\mu<2 / 3$. Assume that $\mathbb{L} \mathbb{C} \mathbb{S}\left(X, B_{X}\right) \neq \varnothing$. Then

- either the set $\mathbb{L} \mathbb{C} S\left(X, B_{X}\right)$ contains a curve,

- or there are a curve $\mathbb{P}^{1} \cong L \subset X$ and a point $P \in L$ such that $L \cdot L=-1$ and

$$
\operatorname{LCS}\left(X, B_{X}\right)=P \text {. }
$$

Proof. Suppose that $\mathbb{L} \mathbb{C S}\left(X, B_{X}\right)$ contains no curves. Then it follows from Theorem 2.7 that

$$
\operatorname{LCS}\left(X, B_{X}\right)=P,
$$

where $P \in X$ is a point. We may assume that $P$ does not lie on any curve $\mathbb{P}^{1} \cong L \subset X$ such that the equality $L \cdot L=-1$ holds. Then there is a birational morphism $\phi: X \longrightarrow \mathbb{P}^{2}$ such that $\phi$ is an isomorphism in a neighborhood of the point $P$. Note that

$$
\phi(P) \in \operatorname{LCS}\left(\mathbb{P}^{2}, \phi\left(B_{X}\right)\right) \neq \varnothing,
$$

the set $\mathbb{L} \mathbb{C S}\left(\mathbb{P}^{2}, \phi\left(B_{X}\right)\right)$ contains no curves, and

$$
\phi\left(B_{X}\right) \sim_{\mathbb{Q}}-\mu K_{\mathbb{P}^{2}} .
$$

Since $\mu<2 / 3$, the latter is impossible by Lemma 2.8 .

Example 5.3. Suppose that $O=\operatorname{Sing}(X)$ and $K_{X}^{2}=5$. Let $\alpha: V \rightarrow X$ be a blow up of $O$, and let $E$ be the exceptional divisor of $\alpha$. Then there is a birational morphism $\omega: V \rightarrow \mathbb{P}^{2}$ such that

- the morphism $\omega$ contracts the curves $E_{1}, E_{2}, E_{3}, E_{4}$,

- the curve $\omega(E)$ is a line in $\mathbb{P}^{2}$ that contains $\omega\left(E_{1}\right), \omega\left(E_{2}\right), \omega\left(E_{3}\right)$, but $\omega(E) \not \ngtr \omega\left(E_{4}\right)$. 
Let $Z$ be a line in $\mathbb{P}^{2}$ such that $\omega\left(E_{1}\right) \in Z \ni \omega\left(E_{4}\right)$. Then

$$
2 E+\bar{Z}+2 E_{1}+E_{2}+E_{3} \sim-K_{V}
$$

where $\bar{Z} \subset V$ is a proper transform of $Z$. One has

$$
\operatorname{lct}\left(X, \alpha\left(\bar{Z}+2 \alpha\left(E_{1}\right)+\alpha\left(E_{2}\right)+\alpha\left(E_{3}\right)\right)=\frac{1}{2},\right.
$$

which implies $\operatorname{lct}(X) \leqslant 1 / 2$. Suppose that $-K_{X} \sim_{\mathbb{Q}} 2 B_{X}$, but $\left(X, B_{X}\right)$ is not log canonical. Then

$$
K_{V}+B_{V}+m E \sim_{\mathbb{Q}} \alpha^{*}\left(K_{X}+B_{X}\right),
$$

for some $m \geqslant 0$, and $B_{V}$ is the proper transform of $B_{X}$ on the surface $V$. Then

$$
\left(V, B_{V}+m E\right)
$$

is not $\log$ canonical at some point $P \in V$. There is a birational morphism $\pi: V \rightarrow U$ such that

- the surface $U$ is a smooth del Pezzo surface with $K_{U}^{2}=6$,

- the morphism $\pi$ is an isomorphism in a neighborhood of $P \in X$, which implies that $\left(U, \pi\left(B_{V}\right)+m \pi(E)\right)$ is not log canonical at $\pi(P)$. But

$$
\pi\left(B_{V}\right)+m \pi(E) \sim_{\mathbb{Q}}-\frac{1}{2} K_{U}
$$

which is impossible, because $\operatorname{lct}(U)=1 / 2$ (see Example 1.18). We see that $\operatorname{lct}(X)=1 / 2$.

Example 5.4. Suppose that $K_{X}^{2}=4$. Arguing as in Example 5.3, we see that the equality

$$
\operatorname{lct}(X)=\left\{\begin{array}{l}
1 / 2 \text { when } O=\operatorname{Sing}(X), \\
2 / 3 \text { when } \operatorname{Sing}(X)=\varnothing
\end{array}\right.
$$

holds (cf. [189]). Take $\lambda \leqslant 1$. Suppose that

$$
B_{X} \sim_{\mathbb{Q}}-K_{X},
$$

and $\left(X, \lambda B_{X}\right)$ is not log canonical at some point $P \in X \backslash O$. There is a commutative diagram

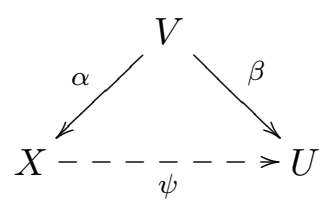

where $U$ is a cubic surface in $\mathbb{P}^{3}$ that has canonical singularities, the morphism $\alpha$ is a blow up of the point $P$, the morphism $\beta$ is birational, and $\psi$ is a projection from the point $P \in X$. Then

$$
K_{V}+\lambda B_{V}+\left(\lambda \operatorname{mult}_{P}\left(B_{X}\right)-1\right) E \sim_{\mathbb{Q}} \alpha^{*}\left(K_{X}+\lambda B_{X}\right),
$$

where $E$ is the exceptional divisor of $\alpha$, and $B_{V}$ is the proper transform of $B_{X}$. Note that

$$
\left(V, \lambda B_{V}+\left(\lambda \operatorname{mult}_{P}\left(B_{X}\right)-1\right) E\right)
$$

is not $\log$ canonical at some point $Q \in E$ and $\operatorname{mult}_{P}\left(B_{X}\right)>1 / \lambda$. Then the log pair

$$
\left(V, \lambda B_{V}+\left(\lambda \operatorname{mult}_{P}\left(B_{X}\right)-\lambda\right) E\right)
$$

is not $\log$ canonical at the point $Q \in E$ as well. But the equivalences

$$
B_{V}+\left(\operatorname{mult}_{P}\left(B_{X}\right)-1\right) E \sim_{\mathbb{Q}}-K_{V}+\alpha^{*}\left(K_{X}+B_{X}\right) \sim_{\mathbb{Q}}-K_{V},
$$

hold. Suppose that $P$ is not contained in any line on the surface $X$. Then

- the morphism $\beta: V \rightarrow U$ is an isomorphism,

- the cubic surface $U$ is smooth outside of the point $\psi(O)$,

- the point $\psi(O)$ is at most ordinary double point of the surface $U$, 
which implies that $\lambda>2 / 3$ (see Example 1.19).

Suppose that $\lambda=3 / 4$. Then the point

$$
\psi(Q) \in U \subset \mathbb{P}^{3}
$$

must be an Eckardt point (see Definition 4.1) of the surface $U$ by Theorem 4.2. But

$$
\beta(E) \subset U \subset \mathbb{P}^{3}
$$

is a line. So, there are two conics $C_{1} \neq C_{2}$ contained in $X$ such that $P=C_{1} \cap C_{2}$ and

$$
C_{1}+C_{2} \sim-K_{X}
$$

Lemma 5.5. Suppose that $O=\operatorname{Sing}(X)$ and $K_{X}^{2}=6$ such that there is a diagram

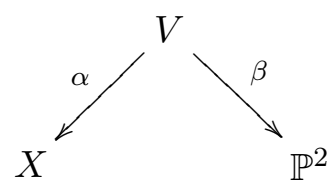

where $\beta$ is a blow up of three points $P_{1}, P_{2}, P_{3} \in \mathbb{P}^{2}$ lying on a line $L \subset \mathbb{P}^{2}$, and $\alpha$ is a birational morphism that contracts an irreducible curve $\bar{L}$ to the point $O$ such that $\beta(\bar{L})=L$. Then

$$
\operatorname{LCS}\left(X, \lambda B_{X}\right)=O
$$

in the case when $\operatorname{LCS}\left(X, \lambda B_{X}\right) \neq \varnothing, B_{X} \sim_{\mathbb{Q}}-K_{X}$ and $\lambda<1 / 2$.

Proof. Suppose that $B_{X} \sim_{\mathbb{Q}}-K_{X}$ and

$$
\varnothing \neq \operatorname{LCS}\left(X, \lambda B_{X}\right) \neq O
$$

let $M \subset \mathbb{P}^{2}$ be a general line, and let $\bar{M} \subset V$ be its proper transform. Then

$$
-K_{X} \sim 2 \alpha(\bar{M})
$$

and $O \in \alpha(\bar{M})$. Thus, the set $\mathbb{L} \mathbb{C S}\left(X, \lambda B_{X}\right)$ contains a curve, because otherwise the locus

$$
\operatorname{LCS}\left(X, \lambda B_{X}+\alpha(\bar{M})\right)
$$

would be disconnected, which is impossible by Theorem 2.7 .

Let $C \subset X$ be an irreducible curve such that $C \subseteq \operatorname{LCS}\left(X, \lambda B_{X}\right)$. Then

$$
B_{X}=\varepsilon C+\Omega \text {, }
$$

where $\varepsilon>2$, and $\Omega$ is an effective $\mathbb{Q}$-divisor such that $C \not \subset \operatorname{Supp}(\Omega)$.

Let $\Gamma_{i} \subset X$ be a proper transform of a general line in $\mathbb{P}^{2}$ that passes through $P_{i}$. Then

$$
O \notin \Gamma_{1} \cup \Gamma_{2} \cup \Gamma_{3}
$$

and $-K_{X} \cdot \Gamma_{1}=-K_{X} \cdot \Gamma_{2}=-K_{X} \cdot \Gamma_{3}=2$. But

$$
-K_{X} \sim_{\mathbb{Q}} \Gamma_{1}+\Gamma_{2}+\Gamma_{3},
$$

which implies that there is $m \in\{1,2,3\}$ such that $C \cdot \Gamma_{m} \neq 0$. Then

$$
2=B_{X} \cdot \Gamma_{m}=(\varepsilon C+\Omega) \cdot \Gamma_{m} \geqslant \varepsilon C \cdot \Gamma_{m} \geqslant \varepsilon>2,
$$

because $\Gamma_{m} \not \subset \operatorname{Supp}\left(B_{X}\right)$. The obtained contradiction completes the proof.

Remark 5.6. Suppose that $O=\operatorname{Sing}(X)$ and $K_{X}^{2}=6$. Let $\alpha: V \rightarrow X$ be a blow up of the point $O \in X$, and let $E$ be the exceptional divisor of $\alpha$. Then

$$
K_{V}+B_{V}+m E \sim_{\mathbb{Q}} \alpha^{*}\left(K_{X}+B_{X}\right)
$$

for some $m \geqslant 0$, and $B_{V}$ is the proper transform of $B_{X}$ on $V$. Note that $\operatorname{lct}(X) \leqslant 1 / 3$. Suppose that $\operatorname{lct}(X)<1 / 3$, i. e. there exists an effective $\mathbb{Q}$-divisor $B_{X} \sim_{\mathbb{Q}}-K_{X}$, such that the $\log$ pair $\left(X, 1 / 3 B_{X}\right)$ is not log canonical. Then the log pair

$$
\left(V, \frac{1}{3}\left(B_{V}+m E\right)\right)
$$


is not $\log$ canonical at some point $P \in V$. There is a birational morphism $\pi: V \longrightarrow U$ such that $U$ is either $\mathbb{F}_{1}$ or $\mathbb{P}^{1} \times \mathbb{P}^{1}$ and $\pi$ is an isomorphism in a neighborhood of $P \in X$. Then the $\log$ pair

$$
\left(U, \frac{1}{3}\left(\pi\left(B_{V}\right)+m \pi(E)\right)\right)
$$

is not $\log$ canonical at the point $\pi(P)$. But we know that

$$
-K_{U} \sim_{\mathbb{Q}} \pi\left(B_{V}\right)+m \pi(E),
$$

so the latter contradicts Example 1.18. Hence $\operatorname{lct}(X)=1 / 3$.

Lemma 5.7. Suppose that $X \cong \mathbb{P}(1,1,2)$ and $B_{X} \sim \mathbb{Q}-K_{X}$, but there is a point $P \in X$ such that

$$
O \neq P \in \operatorname{LCS}\left(X, \lambda B_{X}\right)
$$

for some $\lambda<1 / 2$. Take $L \in\left|\mathcal{O}_{\mathbb{P}(1,1,2)}(1)\right|$ such that $P \in L$. Then $L \subseteq \operatorname{LCS}\left(X, \lambda B_{X}\right)$.

Proof. Suppose that there is a curve $\Gamma \in \operatorname{LCS}\left(X, \lambda B_{X}\right)$ such that $P \in \Gamma \neq L$. Then

$$
B_{X}=\mu \Gamma+\Omega,
$$

where $\mu>2$, and $\Omega$ is an effective $\mathbb{Q}$-divisor such that $\Gamma \not \subset \operatorname{Supp}(\Omega)$. Hence

$$
\mu \Gamma+\Omega \sim_{\mathbb{Q}} 4 L
$$

and $\Gamma \sim m L$, where $m \in \mathbb{Z}_{>0}$. But $m \geqslant 2$, because $P \in \Gamma \neq L$, which is a contradiction.

Suppose that $L \nsubseteq \operatorname{LCS}\left(X, \lambda B_{X}\right)$. Then it follows from Theorem 2.7 that

$$
\operatorname{LCS}\left(X, \lambda B_{X}\right)=P
$$

because we proved that $\mathbb{L} \mathbb{C} S\left(X, \lambda B_{X}\right)$ contains no curves passing through $P \in X$.

Let $C \in\left|\mathcal{O}_{\mathbb{P}(1,1,2)}(1)\right|$ be a general curve. Then

$$
\operatorname{LCS}\left(X, \lambda B_{X}+C\right)=P \cup C,
$$

which is impossible by Theorem 2.7 .

Lemma 5.8. Suppose that $X \cong \mathbb{F}_{1}$. Then there are $0 \leqslant \mu \in \mathbb{Q} \ni \lambda \geqslant 0$ such that

$$
B_{X} \sim_{\mathbb{Q}} \mu C+\lambda L,
$$

where $C$ and $L$ are irreducible curves on the surface $X$ such that

$$
C \cdot C=-1, C \cdot L=1
$$

and $L \cdot L=0$. Suppose that $\mu<1$ and $\lambda<1$. Then $\mathbb{L} \mathbb{C S}\left(X, B_{X}\right)=\varnothing$.

Proof. The set $\mathbb{L} \mathbb{C} S\left(X, B_{X}\right)$ contains no curves, because $L$ and $C$ generate the cone of effective divisors of the surface $X$. Suppose that $\mathbb{L} \mathbb{C S}\left(X, B_{X}\right)$ contains a point $O \in X$. Then

$$
K_{X}+B_{X}+((1-\mu) C+(2-\lambda) L) \sim_{\mathbb{Q}}-(L+C),
$$

because $-K_{X} \sim_{\mathbb{Q}} 2 C+3 L$. But it follows from Theorem 2.6 that the map

$$
0=H^{0}\left(\mathcal{O}_{X}(-L-C)\right) \longrightarrow H^{0}\left(\mathcal{O}_{\mathcal{L}\left(X, B_{X}\right)}\right) \neq 0
$$

is surjective, because the divisor $(1-\mu) C+(2-\lambda) L$ is ample.

Lemma 5.9. Suppose that $\operatorname{Sing}(X)=\varnothing$ and $K_{X}^{2}=7$. Then

$$
L_{1} \cdot L_{1}=L_{2} \cdot L_{2}=L_{3} \cdot L_{3}=-1, L_{1} \cdot L_{2}=L_{2} \cdot L_{3}=1, L_{1} \cdot L_{3}=0
$$

where $L_{1}, L_{2}, L_{3}$ are exceptional curves on $X$. Suppose that $\operatorname{LCS}\left(X, B_{X}\right) \neq \varnothing$ and

$$
B_{X} \sim_{\mathbb{Q}}-\mu K_{X}
$$

where $\mu<1 / 2$. Then $\operatorname{LCS}\left(X, B_{X}\right)=L_{2}$. 
Proof. Let $P$ be a point in $\operatorname{LCS}\left(X, B_{X}\right)$. Then $P \in L_{2}$, because $\operatorname{lct}\left(\mathbb{P}^{1} \times \mathbb{P}^{1}\right)=1 / 2$, and there is a birational morphism $X \rightarrow \mathbb{P}^{1} \times \mathbb{P}^{1}$ that contracts only the curve $L_{2}$.

Suppose that $\operatorname{LCS}\left(X, B_{X}\right) \neq L_{2}$. Then $\operatorname{LCS}\left(X, B_{X}\right)=P$ by Theorem 2.7.

We may assume that $P \notin L_{3}$. There is a birational morphism $\phi: X \rightarrow \mathbb{P}^{2}$ that contracts the curves $L_{1}$ and $L_{3}$. Let $C_{1}$ and $C_{3}$ be proper transforms on $X$ of sufficiently general lines in $\mathbb{P}^{2}$ that pass through the points $\phi\left(L_{1}\right)$ and $\phi\left(L_{3}\right)$, respectively. Then

$$
-K_{X} \sim C_{1}+2 C_{3}+L_{3}
$$

and $C_{1} \not \ngtr P \notin C_{2}$. Therefore, we see that

$$
C_{2} \cup P \subseteq \operatorname{LCS}\left(X, \lambda D+\frac{1}{2}\left(C_{1}+2 C_{2}+L_{3}\right)\right) \subseteq C_{2} \cup P \cup L_{3},
$$

which is impossible by Theorem 2.7, because $P \notin L_{3}$.

Lemma 5.10. Suppose that $O=\operatorname{Sing}(X)$, the equality $K_{X}^{2}=7$ holds, the equivalence

$$
B_{X} \sim_{\mathbb{Q}} C+\frac{4}{3} L
$$

holds, where $L \cong \mathbb{P}^{1} \cong C$ are curves on the surface $X$ such that

$$
L \cdot L=-1 / 2, C \cdot C=-1, C \cdot L=1,
$$

but the log pair $\left(X, B_{X}\right)$ is not $\log$ canonical at some point $P \in C$. Then $P \in L$.

Proof. Let $S$ be a quadratic cone in $\mathbb{P}^{3}$. Then $S \cong \mathbb{P}(1,1,2)$ and there is a birational morphism

$$
\phi: X \longrightarrow S \subset \mathbb{P}^{3}
$$

that contracts the curve $C$ to a smooth point $Q \in S$. Then $Q \in \phi(L) \in\left|\mathcal{O}_{\mathbb{P}(1,1,2)}(1)\right|$.

Suppose that $P \notin L$. Then it follows from Remark 2.23 that to complete the proof we may assume that either $C \not \subset \operatorname{Supp}\left(B_{X}\right)$ or $L \not \subset \operatorname{Supp}\left(B_{X}\right)$, because the log pair

$$
\left(X, C+\frac{4}{3} L\right)
$$

is $\log$ canonical in the point $P \in X$. Suppose that $C \not \subset \operatorname{Supp}\left(B_{X}\right)$. Then

$$
\frac{1}{3}=B_{X} \cdot C \geqslant \operatorname{mult}_{P}\left(B_{X}\right)>1
$$

which is impossible. Therefore, we see that $C \subset \operatorname{Supp}\left(B_{X}\right)$. Then $L \not \subset \operatorname{Supp}\left(B_{X}\right)$. Put

$$
B_{X}=\varepsilon C+\Omega,
$$

where $\Omega$ is an effective $\mathbb{Q}$-divisor such that $C \not \subset \operatorname{Supp}(\Omega)$. Then

$$
\frac{1}{3}=B_{X} \cdot L=\varepsilon+\Omega \cdot L \geqslant \varepsilon,
$$

which implies that $\varepsilon \leqslant 1 / 3$. Then

$$
1<\Omega \cdot C=1 / 3+\varepsilon \leqslant 2 / 3
$$

by Lemma 2.21, which is a contradiction.

\section{TORIC VARIETIES}

The purpose of this section is to prove Lemma 6.1 (cf. [7], [174, [39]).

Let $N=\mathbb{Z}^{n}$ be a lattice of rank $n$, and let $M=\operatorname{Hom}(N, \mathbb{Z})$ be the dual lattice. Put $M_{\mathbb{R}}=M \otimes_{\mathbb{Z}} \mathbb{R}$ and $N_{\mathbb{R}}=N \otimes_{\mathbb{Z}} \mathbb{R}$. Let $X$ be a toric variety defined by a complete fan $\Sigma \subset N_{\mathbb{R}}$, let

$$
\Delta_{1}=\left\{v_{1}, \ldots, v_{m}\right\}
$$

be a set of generators of one-dimensional cones of the fan $\Sigma$. Put

$$
\Delta=\left\{w \in M \mid\left\langle w, v_{i}\right\rangle \geqslant-1 \text { for all } i=1, \ldots, m\right\} .
$$

Put $T=\left(\mathbb{C}^{*}\right)^{n} \subset \operatorname{Aut}(X)$. Let $\mathcal{N}$ be the normalizer of $T$ in $\operatorname{Aut}(X)$ and $\mathcal{W}=\mathcal{N} / T$. 
Lemma 6.1. Let $G \subset \mathcal{W}$ be a subgroup. Suppose that $X$ is $\mathbb{Q}$-factorial. Then

$$
\operatorname{lct}(X, G)=\frac{1}{1+\max \left\{\langle w, v\rangle \mid w \in \Delta^{G}, v \in \Delta_{1}\right\}},
$$

where $\Delta^{G}$ is the set of the points in $\Delta$ that are fixed by the group $G$.

Proof. Put $\mu=1+\max \left\{\langle w, v\rangle \mid w \in \Delta^{G}, v \in \Delta_{1}\right\}$. Then $\mu \in \mathbb{Q}$ is the largest number such that

$$
-K_{X} \sim_{\mathbb{Q}} \lambda R+H
$$

where $R$ is an integral $T \rtimes G$-invariant effective divisor, and $H$ is an effective $\mathbb{Q}$-divisor. Hence

$$
\operatorname{lct}(X, G) \leqslant \frac{1}{\mu}
$$

Suppose that $\operatorname{lct}(X, G)<1 / \mu$. Then there is an effective $G$-invariant $\mathbb{Q}$-divisor $D \sim_{\mathbb{Q}}-K_{X}$ such that the log pair $(X, \lambda D)$ is not $\log$ canonical for some rational $\lambda<1 / \mu$.

There exists a family $\left\{D_{t} \mid t \in \mathbb{C}\right\}$ of $G$-invariant effective $\mathbb{Q}$-divisors such that

- the equivalence $D_{t} \sim_{\mathbb{Q}} D$ holds for every $t \in \mathbb{C}$,

- the equality $D_{1}=D$ holds,

- for every $t \neq 0$ there is $\phi_{t} \in \operatorname{Aut}(X)$ such that

$$
D_{t}=\phi_{t}(D) \cong D
$$

- the divisor $D_{0}$ is $T$-invariant,

which implies that $\left(X, \lambda D_{0}\right)$ is not log canonical (see [49]).

On the other hand, the divisor $D_{0}$ does not have components with multiplicity greater then $\mu$, which implies that $\left(X, \lambda D_{0}\right)$ is log canonical (see [61, [121]), which is a contradiction.

Corollary 6.2. Let $X=\mathbb{P}_{\mathbb{P}^{n}}\left(\mathcal{O}_{\mathbb{P}^{n}} \oplus \mathcal{O}_{\mathbb{P}^{n}}\left(-a_{1}\right) \oplus \ldots \oplus \mathcal{O}_{\mathbb{P}^{n}}\left(-a_{k}\right)\right), a_{i} \geqslant 0$ for $i=1, \ldots, k$. Then

$$
\operatorname{lct}(X)=\frac{1}{1+\max \left\{k, n+\sum_{i=1}^{k} a_{i}\right\}} .
$$

Proof. Note that $X$ is a toric variety, and $\Delta_{1}$ consists of the following vectors:

$$
\begin{gathered}
(\overbrace{1,0, \ldots, 0}^{k}, \overbrace{0,0, \ldots, 0}^{n}), \\
\ldots \\
(0, \ldots, 0,1,0,0, \ldots, 0), \\
(-1, \ldots,-1,0,0, \ldots, 0), \\
(0,0, \ldots, 0,1,0, \ldots, 0), \\
\ldots \\
(0,0, \ldots, 0,0, \ldots, 0,1), \\
\left(-a_{1}, \ldots,-a_{k},-1, \ldots,-1\right),
\end{gathered}
$$

which implies the required assertion by Lemma 6.1.

Applying Corollary 6.2, we obtain the following result.

Corollary 6.3. In the notation of section 1 one has

$$
\operatorname{lct}(X)=\left\{\begin{array}{l}
1 / 4 \text { whenever } \beth(X) \in\{2.33,2.35\}, \\
1 / 5 \text { whenever } \beth(X)=2.36
\end{array}\right.
$$

On the other hand, straightforward computations using Lemma 6.1 imply the following result.

Corollary 6.4. In the notation of section 1 one has

$$
\operatorname{lct}(X)=\left\{\begin{array}{l}
1 / 3 \text { whenever } \exists(X) \in\{3.25,3.31,4.9,4.11,5.2\}, \\
1 / 4 \text { whenever } \Xi(X) \in\{3.26,3.30,4.12\}, \\
1 / 5 \text { whenever } \Xi(X)=3.29 .
\end{array}\right.
$$


Another application of Lemma 6.1 is an immediate proof of the following result, obtained in [77].

Example 6.5. Let $X$ be a blow up of $\mathbb{P}^{n}, n \geqslant 2$, at $k+1$ points that are not contained in a linear subspace of dimension $k-1$. Assume that $k \leqslant n$. Then the points are in general position (i. e. no $r+1$ of them are contained in a linear subspace of dimension $r-1$ ), so that $X$ is toric, and

$$
\operatorname{lct}(X)=\frac{1}{n+1}
$$

by Lemma 6.1. Note that $X$ is a Fano variety only if $k=1$, or $n=2$ and $k \leqslant 3$ (cf. [77, Chapter 5]).

Remark 6.6. Let $X$ be a blow up of $\mathbb{P}^{n}, n \geqslant 2$, at $n+1$ points that are not contained in a linear subspace of dimension $n-1$. Then $X$ is not Fano whether $n \geqslant 3$, but the points are still in general position, so that $X$ is toric, and

$$
\operatorname{lct}(X)=\frac{1}{n}
$$

by Lemma 6.1. On the other hand, there is a natural action of the symmetric group $S_{n+1}$ on $X$ such that

$$
\operatorname{lct}\left(X, S_{n+1}\right)=1 .
$$

\section{Del Pezzo threefolds}

We use the assumptions and notation of Theorem 1.78, Suppose that $-K_{X} \sim 2 H$, where $H$ is a Cartier divisor that is indivisible in $\operatorname{Pic}(X)$.

The purpose of this section is to prove the following result.

Theorem 7.1. The equality $\operatorname{lct}(X)=1 / 2$ holds, unless $\beth(X)=2.35$ when $\operatorname{lct}(X)=1 / 4$.

It follows from Theorems 3.1.14 and 3.3.1 in [98] that

$$
I(X) \in\{1.11,1.12,1.13,1.14,1.15,1.17,2.32,2.35,3.27\},
$$

and by [20] and [28] (see also Lemma 2.18) one has $\operatorname{lct}(X)=1 / 2$ if $I(X) \in\{1.12,1.13\}$.

It follows from Lemma 2.30 that $\operatorname{lct}(X)=1 / 2$ when $I(X)=3.27$.

Lemma 7.2. Suppose that $I(X)=2.35$. Then $\operatorname{lct}(X)=1 / 4$.

Proof. There is a birational morphism $\pi: X \rightarrow \mathbb{P}^{3}$ that contracts a surface $E \cong \mathbb{P}^{2}$ to a point $P \in \mathbb{P}^{3}$. Hence $\operatorname{lct}(X) \leqslant 1 / 4$, because

$$
-K_{X} \sim 2 E+4 T
$$

where $T$ is the proper transform of a plane in $\mathbb{P}^{3}$ that passes through $P \in \mathbb{P}^{3}$.

Suppose that $\operatorname{lct}(X)<1 / 4$. Then there is an effective $\mathbb{Q}$-divisor $D$ such that

$$
D \sim_{\mathbb{Q}} \frac{1}{2} E+T
$$

but the log pair $(X, \lambda D)$ is not $\log$ canonical for some rational number $\lambda<1$.

Let $R$ be a proper transform on $X$ of a general plane in $\mathbb{P}^{3}$. Then

$$
\operatorname{LCS}(X, \lambda D+R)
$$

must be connected by Theorem 2.7, because $-\left(K_{X}+\lambda D+R\right)$ is ample. Thus, we see that

- the subscheme $\mathcal{L}(X, \lambda D)$ is not zero-dimensional,

- the locus $\operatorname{LCS}(X, \lambda D)$ is not contained in $E$,

which implies that $\left(\mathbb{P}^{3}, \lambda \pi(D)\right)$ is not $\log$ canonical. But $\operatorname{lct}\left(\mathbb{P}^{3}\right)=1 / 4$ and $\pi(D) \sim_{\mathbb{Q}} \mathcal{O}_{\mathbb{P}^{3}}(1)$, which is a contradiction.

Remark 7.3. Actually, the assertion of Lemma 7.2 is contained in Corollary 6.3, but we still prefer to give a detailed proof that may have further applications. 
We are left with the cases

$$
J(X) \in\{1.11,1.14,1.15,2.32\},
$$

while the inequality $\operatorname{lct}(X) \leqslant 1 / 2$ is obvious, because $|H|$ is not empty.

Lemma 7.4. Suppose that $I(X)=2.32$. Then $\operatorname{lct}(X)=1 / 2$.

Proof. We may suppose that $\operatorname{lct}(X)<1 / 2$. Then there is an effective $\mathbb{Q}$-divisor $D \sim_{\mathbb{Q}} H$ such that the log pair $(X, \lambda D)$ is not $\log$ canonical for some positive rational number $\lambda<1$.

The threefold $X$ is a divisor on $\mathbb{P}^{2} \times \mathbb{P}^{2}$ of bi-degree $(1,1)$. There are two natural $\mathbb{P}^{1}$-bundles $\pi_{1}: X \rightarrow \mathbb{P}^{2}$ and $\pi_{2}: X \rightarrow \mathbb{P}^{2}$, and applying Theorem 2.28 to $\pi_{1}$ and $\pi_{2}$ we immediately obtain a contradiction.

Remark 7.5. Suppose that $\operatorname{Pic}(X)=\mathbb{Z}[H]$, and there is an effective $\mathbb{Q}$-divisor $D \sim_{\mathbb{Q}} H$ such that the log pair $(X, \lambda D)$ is not log canonical for some positive rational number $\lambda<1$. Put

$$
D=\varepsilon S+\Omega \sim \sim_{\mathbb{Q}} H,
$$

where $S$ is an irreducible surface and $\Omega$ is an effective $\mathbb{Q}$-divisor such that

$$
\operatorname{Supp}(\Omega) \not \supset S .
$$

Then $\varepsilon \leqslant 1$, because $\operatorname{Pic}(X)=\mathbb{Z}[H]$, which implies that the set $\mathbb{L} \mathbb{C} S(X, \lambda D)$ contains no surfaces. Moreover, for any choice of $H \in|H|$ the locus

$$
\operatorname{LCS}(X, \lambda D+H)
$$

is connected by Theorem 2.7. Let $H$ be a general surface in $|H|$. Since $\operatorname{LCS}(X, \lambda D+H)$ is connected, one obtains that the $\operatorname{locus} \operatorname{LCS}(X, \lambda D+H)$ has no isolated zero-dimensional components outside the base locus of the linear system $|H|$. Note that $|H|$ has no base points at all, unless $\mathrm{I}(X)=1.11$ when $\mathrm{Bs}|H|$ consists of a single point $O$. Note that in the latter case $O \notin \operatorname{LCS}(X, \lambda D)$, since $X$ is covered by the curves of anticanonical degree 2 passing through $O$. Hence the locus $\operatorname{LCS}(X, \lambda D)$ never has isolated zero-dimensional components; in particular, it contains an (irreducible) curve $C$. Put $\left.D\right|_{H}=\bar{D}$. Then

$$
-\left.K_{H} \sim H\right|_{H} \sim_{\mathbb{Q}} \bar{D}
$$

but $(H, \lambda \bar{D})$ is not log canonical in every point of the intersection $H \cap C$. The locus $\operatorname{LCS}(H, \lambda \bar{D})$ is connected by Theorem 2.7. But the scheme $\mathcal{L}(H, \lambda \bar{D})$ is zero-dimensional. We see that

$$
H \cdot C=|H \cap C|=1,
$$

and the locus $\operatorname{LCS}(X, \lambda D)$ contains no curves besides the irreducible curve $C$.

Lemma 7.6. Suppose that $I(X)=1.15$. Then $\operatorname{lct}(X)=1 / 2$.

Proof. We may suppose that $\operatorname{lct}(X)<1 / 2$. Then there is an effective $\mathbb{Q}$-divisor $D \sim \mathbb{Q} H$ such that the log pair $(X, \lambda D)$ is not $\log$ canonical for some positive rational number $\lambda<1$.

The linear system $|H|$ induces an embedding $X \subset \mathbb{P}^{6}$. Thus, it follows from Remark [7.5 that the locus $\operatorname{LCS}(X, \lambda D)$ consists of a single line $C \subset X \subset \mathbb{P}^{6}$.

It follows from [98, Proposition 3.4.1] (see [92] and 67]) that there is a commutative diagram

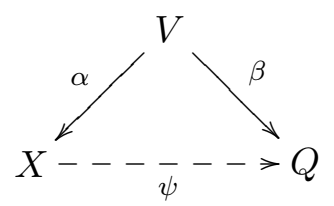

where $Q$ is a quadric in $\mathbb{P}^{4}$, the morphism $\alpha$ is a blow up of $C$, the morphism $\beta$ is a blow up of a smooth rational cubic curve $Z \subset Q$, and the map $\psi$ is a projection from the line $C$.

Let $S$ be the exceptional divisor of $\beta$, and let $L$ be a fiber of the morphism $\beta$ over a general point of the curve $Z$. Put $\bar{S}=\alpha(S)$ and $\bar{L}=\alpha(L)$. Then $\bar{S} \sim H$, the curve $\bar{L}$ is a line, and the surface $\bar{S}$ is singular along $C$. Moreover, the singularity of $\bar{S}$ at a general point of $C$ is locally isomorphic to $T \times \mathbb{A}^{1}$, where $T$ is a germ of a nodal curve. In particular, the pair $(X, \bar{S})$ is $\log$ canonical. 
We may assume that $\operatorname{Supp}(D) \not \supset \bar{S}$ by Remark 2.23. Then

$$
1=\bar{L} \cdot D \geqslant \operatorname{mult}_{C}(D)>1 / \lambda>1 \text {, }
$$

which is a contradiction.

Lemma 7.7. Let $I(X)=1.14$. Then $\operatorname{lct}(X)=1 / 2$.

Proof. We may suppose that $\operatorname{lct}(X)<1 / 2$. Then there is an effective $\mathbb{Q}$-divisor $D \sim_{\mathbb{Q}} H$ such that the log pair $(X, \lambda D)$ is not $\log$ canonical for some positive rational number $\lambda<1$.

The linear system $|H|$ induces an embedding $X \subset \mathbb{P}^{5}$ such that $X$ is a complete intersection of two quadrics. Then the locus $\operatorname{LCS}(X, \lambda D)$ consists of a single line $C \subset X \subset \mathbb{P}^{5}$ by Remark 7.5.

It follows from [98, Proposition 3.4.1] that there is a commutative diagram

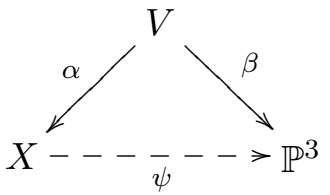

where $\psi$ is a projection from $C$, the morphism $\alpha$ is a blow up of the line $C$, and $\beta$ is a blow up of a smooth curve $Z \subset \mathbb{P}^{3}$ of degree 5 and genus 2 .

Let $S$ be the exceptional divisor of $\beta$, and let $L$ be a fiber of the morphism $\beta$ over a general point of the curve $Z$. Put $\bar{S}=\alpha(S)$ and $\bar{L}=\alpha(L)$. Then $\bar{S} \sim 2 H$, the curve $\bar{L}$ is a line, and $\operatorname{mult}_{C}(\bar{S})=3$. But the log pair $(X, 1 / 2 \bar{S})$ is log canonical, which implies that we may assume that $\operatorname{Supp}(D) \not \supset \bar{S}$ by Remark 2.23 . Then

$$
1=\bar{L} \cdot D \geqslant \operatorname{mult}_{C}(D)>1 / \lambda>1,
$$

which is a contradiction.

Remark 7.8. Let $V \subset \mathbb{P}^{5}$ be a complete intersection of two quadric hypersurfaces that has isolated singularities, and let $B_{V}$ be an effective $\mathbb{Q}$-divisor on $V$ such that $B_{V} \sim_{\mathbb{Q}}-K_{V}$ and

$$
\operatorname{LCS}\left(V, \mu B_{V}\right) \neq \varnothing,
$$

where $\mu<1 / 2$. Arguing as in the proof of Lemma 7.7, we see that

$$
\operatorname{LCS}\left(V, \mu B_{V}\right) \subseteq L
$$

where $L \subset V$ is a line such that $L \cap \operatorname{Sing}(V) \neq \varnothing$.

Lemma 7.9. Suppose that $I(X)=1$.11. Then $\operatorname{lct}(X)=1 / 2$.

Proof. We may suppose that $\operatorname{lct}(X)<1 / 2$. Then there is an effective $\mathbb{Q}$-divisor $D \sim_{\mathbb{Q}} H$ such that the log pair $(X, \lambda D)$ is not $\log$ canonical for some positive rational number $\lambda<1$.

Recall that the threefold $X$ can be given by an equation

$$
w^{2}=t^{3}+t^{2} f_{2}(x, y, z)+t f_{4}(x, y, z)+f_{6}(x, y, z) \subset \mathbb{P}(1,1,1,2,3) \cong \operatorname{Proj}(\mathbb{C}[x, y, z, t, w]),
$$

where $\operatorname{wt}(x)=\operatorname{wt}(y)=\operatorname{wt}(z)=1, \operatorname{wt}(t)=2, \operatorname{wt}(w)=3$, and $f_{i}$ is a polynomial of degree $i$.

The locus $\operatorname{LCS}(X, \lambda D)$ consists of a single curve $C \subset X$ such that $H \cdot C=1$ by Remark 7.5.

Let $\psi: X \rightarrow \mathbb{P}^{2}$ be the natural projection. Then $\psi$ is not defined in a point $O$ that is cut out by $x=y=z=0$. The curve $C$ does not contain the point $O$, because otherwise we get

$$
1=\Gamma \cdot D \geqslant \operatorname{mult}_{O}(D) \operatorname{mult}_{O}(\Gamma) \geqslant \operatorname{mult}_{C}(D)>1 / \lambda>1,
$$

where $\Gamma$ is a general fiber of the projection $\psi$. Thus, we see that $\psi(C) \subset \mathbb{P}^{2}$ is a line.

Let $S$ be the unique surface in $|H|$ such that $C \subset S$. Let $L$ be a sufficiently general fiber of the rational map $\psi$ that intersects the curve $C$. Then $L \subset \operatorname{Supp}(D)$, since otherwise

$$
1=D \cdot L \geqslant \operatorname{mult}_{C}(D)>1 / \lambda>1 \text {. }
$$

We may assume that $D=S$ by Remark 2.23 . Then $S$ has a cuspidal singularity along $C$. 
We may assume that the surface $S$ is cut out on $X$ by the equation $x=0$, and we may assume that the curve $C$ is given by $w=t=x=0$. Then $S$ is given by

$$
w^{2}=t^{3}+t^{2} f_{2}(0, y, z)+t f_{4}(0, y, z) \subset \mathbb{P}(1,1,2,3) \cong \operatorname{Proj}(\mathbb{C}[y, z, t, w]),
$$

and $f_{6}(x, y, z)=x f_{5}(x, y, z)$, where $f_{5}(x, y, z)$ is a homogeneous polynomial of degree 5 .

Since the surface $S$ is singular along the curve $C$, one has

$$
f_{4}(x, y, z)=x f_{3}(x, y, z),
$$

where $f_{3}(x, y, z)$ is a homogeneous polynomial of degree 3 . Then every point of the set

$$
x=f_{5}(x, y, z)=t=w=0 \subset \mathbb{P}(1,1,1,2,3)
$$

must be singular on $X$, which is a contradiction, because $X$ is smooth.

The assertion of Theorem 7.1 is completely proved.

\section{FANO THREEFOLDS With $\rho=2$}

We use the assumptions and notation introduced in section 1 .

Lemma 8.1. Suppose that $I(X)=2.1$ or $I(X)=2.3$. Then $\operatorname{lct}(X)=1 / 2$.

Proof. There is a birational morphism $\alpha: X \rightarrow V$ that contracts a surface $E \subset X$ to a smooth elliptic curve $C \subset V$, where $V$ is one of the following Fano threefolds:

- smooth hypersurface in $\mathbb{P}(1,1,1,2,3)$ of degree 6 ;

- smooth hypersurface in $\mathbb{P}(1,1,1,1,2)$ of degree 4 .

The curve $C$ is contained in a surface $H \subset V$ such that

$$
\operatorname{Pic}(V)=\mathbb{Z}[H]
$$

and $-K_{X} \sim 2 H$. Then $C$ is a complete intersection of two surfaces in $|H|$, and

$$
-K_{X} \sim 2 \bar{H}+E,
$$

where $E$ is the exceptional divisor of the birational morphism $\alpha$, and $\bar{H}$ is a proper transform of the surface $H$ on the threefold $X$. In particular, the inequality $\operatorname{lct}(X) \leqslant 1 / 2$ holds.

We suppose that $\operatorname{lct}(X)<1 / 2$. Then there exists an effective $\mathbb{Q}$-divisor $D \sim_{\mathbb{Q}}-K_{X}$ such that the $\log$ pair $(X, \lambda D)$ is not log canonical for some positive rational number $\lambda<1 / 2$. Then

$$
\varnothing \neq \operatorname{LCS}(X, \lambda D) \subseteq E,
$$

since $\operatorname{lct}(V)=1 / 2$ by Theorem 7.1 and $\alpha(D) \sim_{\mathbb{Q}} 2 H \sim-K_{V}$.

Put $k=H \cdot C$. Then $k=H^{3} \in\{1,2\}$. Note that

$$
\mathcal{N}_{C / V} \cong \mathcal{O}_{C}\left(\left.H\right|_{C}\right) \oplus \mathcal{O}_{C}\left(\left.H\right|_{C}\right),
$$

which implies that $E \cong C \times \mathbb{P}^{1}$. Let $Z \cong C$ and $L \cong \mathbb{P}^{1}$ be curves on $E$ such that

$$
Z \cdot Z=L \cdot L=0
$$

and $Z \cdot L=1$. Then $\left.\alpha^{*}(H)\right|_{E} \sim k L$, and since

$$
-\left.\left.2 Z \sim K_{E} \sim\left(K_{X}+E\right)\right|_{E} \sim\left(2 E-2 \alpha^{*}(H)\right)\right|_{E} \sim-2 k L+\left.2 E\right|_{E},
$$

we see that $\left.E\right|_{E} \sim-Z+k L$. Put

$$
D=\mu E+\Omega,
$$

where $\Omega$ is an effective $\mathbb{Q}$-divisor on $X$ such that $E \not \subset \operatorname{Supp}(\Omega)$.

The pair $(X, E+\lambda \Omega)$ is not log canonical in the neighborhood of $E$. Hence, the log pair

$$
\left(E,\left.\lambda \Omega\right|_{E}\right)
$$

is also not log canonical by Theorem 2.20. But

$$
\left.\left.\left.\Omega\right|_{E} \sim_{\mathbb{Q}}\left(-K_{X}-\mu E\right)\right|_{E} \sim_{\mathbb{Q}}\left(2 \alpha^{*}(H)-(1+\mu) E\right)\right|_{E} \sim_{\mathbb{Q}}(1+\mu) Z+k(1-\mu) L,
$$

and $0 \leqslant \lambda k(1-\mu) \leqslant 1$, which contradicts Lemma 2.24 . 
Lemma 8.2. Suppose that $I(X)=2.4$ and $X$ is general. Then $\operatorname{lct}(X)=3 / 4$.

Proof. There is a commutative diagram

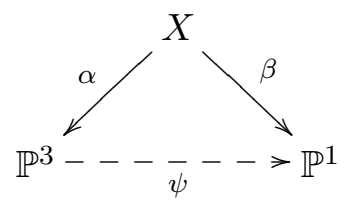

where $\psi$ is a rational map, $\alpha$ is a blow up of a smooth curve $C \subset \mathbb{P}^{3}$ such that

$$
C=H_{1} \cdot H_{2}
$$

for some $H_{1}, H_{2} \in\left|\mathcal{O}_{\mathbb{P}^{3}}(3)\right|$, and $\beta$ is a fibration into cubic surfaces.

Let $\mathcal{P}$ be a pencil in $\left|\mathcal{O}_{\mathbb{P}^{3}}(3)\right|$ generated by $H_{1}$ and $H_{2}$. Then $\psi$ is given by $\mathcal{P}$.

We assume that $X$ satisfies the following generality conditions:

- every surface in $\mathcal{P}$ has at most one ordinary double point;

- the curve $C$ contains no Eckardt point:7 (see Definition 4.1) of any surface in $\mathcal{P}$.

Let $E$ be the exceptional divisor of the birational morphism $\alpha$. Then

$$
\frac{4}{3} \bar{H}_{1}+\frac{1}{3} E \sim_{\mathbb{Q}} \frac{4}{3} \bar{H}_{2}+\frac{1}{3} E \sim_{\mathbb{Q}}-K_{X},
$$

where $\bar{H}_{i}$ is a proper transform of $H_{i}$ on the threefold $X$. We see that $\operatorname{lct}(X) \leqslant 3 / 4$.

We suppose that $\operatorname{lct}(X)<3 / 4$. Then there exists an effective $\mathbb{Q}$-divisor $D \sim_{\mathbb{Q}}-K_{X}$ such that the log pair $(X, \lambda D)$ is not log canonical for some positive rational number $\lambda<3 / 4$.

Suppose that the set $\mathbb{L} \mathbb{C} S(X, \lambda D)$ contains a (irreducible) surface $S \subset X$. Then

$$
D=\varepsilon S+\Delta,
$$

where $\varepsilon \geqslant 1 / \lambda$, and $\Delta$ is an effective $\mathbb{Q}$-divisor such that $S \not \subset \operatorname{Supp}(\Delta)$. Then

$$
\left(\bar{H}_{1},\left.D\right|_{\bar{H}_{1}}\right)
$$

is not $\log$ canonical by Remark 2.3 if $S \cap \bar{H}_{1} \neq \varnothing$. But

$$
\left.D\right|_{\bar{H}_{1}} \sim_{\mathbb{Q}}-K_{\bar{H}_{1}} .
$$

We can choose $\bar{H}_{1}$ to be a smooth cubic surface in $\mathbb{P}^{3}$. Thus, it follows from Theorem 4.2 that

$$
S \cap \bar{H}_{1}=\varnothing,
$$

which implies that $S \sim \bar{H}_{1}$. Thus, we see that $\alpha(S)$ is a cubic surface in $\mathcal{P}$. Then

$$
\varepsilon \alpha(S)+\alpha(\Delta) \sim_{\mathbb{Q}} \mathcal{O}_{\mathbb{P}^{3}}(4),
$$

which is impossible, because $\varepsilon \geqslant 1 / \lambda>4 / 3$.

Let $F$ be a fiber of $\beta$ such that $F \cap \operatorname{LCS}(X, \lambda D) \neq \varnothing$. Put

$$
D=\mu F+\Omega,
$$

where $\Omega$ is an effective $\mathbb{Q}$-divisor such that $F \not \subset \operatorname{Supp}(\Omega)$. Then the log pair $\left(F,\left.\lambda \Omega\right|_{F}\right)$ is not log canonical by Theorem 2.20, because $\lambda \mu<1$. It follows from Theorem 4.2 that

$$
\operatorname{LCS}\left(F,\left.\lambda \Omega\right|_{F}\right)=O
$$

where either $O$ is an Eckardt point of the surface $F$, or $O=\operatorname{Sing}(F)$. By Theorem 2.7

$$
\operatorname{LCS}(X, \lambda D)=\operatorname{LCS}(X, \lambda \mu F+\lambda \Omega D)=O,
$$

because it follows from Theorem 2.20 that $(X, F+\lambda \Omega D)$ is not $\log$ canonical at $O$ and is $\log$ canonical in a punctured neighborhood of $O$. But $O \notin E$ by our generality assumptions. Then

$$
\alpha(O) \subset \operatorname{LCS}\left(\mathbb{P}^{3}, \lambda \alpha(D)\right) \subseteq \alpha(O) \cup C,
$$

\footnotetext{
${ }^{7}$ Note that $C$ does not contain singular points of the surfaces in $\mathcal{P}$ since $C$ is a complete intersection of two surfaces from $\mathcal{P}$.
} 
where $\alpha(O) \notin C$. But $\lambda<3 / 4$, which contradicts Lemma 2.8 .

Lemma 8.3. Suppose that $I(X) \in\{2.5,2.10,2.14\}$ and $X$ is general. Then $\operatorname{lct}(X)=1 / 2$.

Proof. There is a commutative diagram

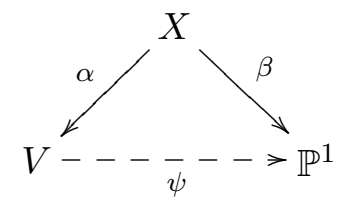

where $V$ is a smooth Fano threefold such that $-K_{V} \sim 2 H$ for some $H \in \operatorname{Pic}(V)$ and

$$
I(V) \in\{1.13,1.14,1.15\},
$$

the morphism $\alpha$ is a blow up of a smooth curve $C \subset V$ such that

$$
C=H_{1} \cdot H_{2}
$$

for some $H_{1}, H_{2} \in|H|, H_{1} \neq H_{2}$, the morphism $\beta$ is a del Pezzo fibration, and $\psi$ is a linear projection.

Let $E$ be the exceptional divisor of the birational morphism $\alpha$. Then

$$
2 \bar{H}_{1}+E \sim 2 \bar{H}_{2}+E \sim-K_{X},
$$

where $\bar{H}_{i}$ is a proper transform of $H_{i}$ on the threefold $X$. We see that $\operatorname{lct}(X) \leqslant 1 / 2$.

We suppose that $\operatorname{lct}(X)<1 / 2$. Then there exists an effective $\mathbb{Q}$-divisor $D \sim_{\mathbb{Q}}-K_{X}$ such that the log pair $(X, \lambda D)$ is not log canonical for some positive rational number $\lambda<1 / 2$. Then

$$
\varnothing \neq \operatorname{LCS}(X, \lambda D) \subseteq E,
$$

because $\alpha(D) \sim_{\mathbb{Q}}-K_{V}$ and $\operatorname{lct}(V)=1 / 2$ by Theorem 7.1 .

We assume that the threefold $X$ satisfies the following generality condition: every fiber of the del Pezzo fibration $\beta$ has at most one singular point that is an ordinary double point.

Let $F$ be a fiber of $\beta$ such that $F \cap \operatorname{LCS}(X, \lambda D) \neq \varnothing$. Put

$$
D=\mu F+\Omega,
$$

where $\Omega$ is an effective $\mathbb{Q}$-divisor on $X$ such that $F \not \subset \operatorname{Supp}(\Omega)$. Then

$$
\alpha(D)=\mu \alpha(F)+\alpha(\Omega) \sim_{\mathbb{Q}} 2 \alpha(F) \sim_{\mathbb{Q}}-K_{V},
$$

which implies that $\mu \leqslant 2$. Then $\left(F,\left.\lambda \Omega\right|_{F}\right)$ is also not log canonical by Theorem 2.20 , But

$$
\left.\Omega\right|_{F} \sim \mathbb{Q}-K_{F},
$$

which implies that $\operatorname{lct}(F) \leqslant \lambda<1 / 2$. But $F$ has at most one ordinary double point and

$$
K_{F}^{2}=H^{3} \leqslant 5,
$$

which implies that $\operatorname{lct}(F) \geqslant 1 / 2$ (see Examples 1.18, 1.19, 5.3 and 5.4), which is a contradiction.

Lemma 8.4. Suppose that $I(X)=2.8$ and $X$ is general. Then $\operatorname{lct}(X)=1 / 2$.

Proof. Let $O \in \mathbb{P}^{3}$ be a point, and let $\alpha: V_{7} \rightarrow \mathbb{P}^{3}$ be a blow up of the point $O$. Then

$$
V_{7} \cong \mathbb{P}\left(\mathcal{O}_{\mathbb{P}^{2}} \oplus \mathcal{O}_{\mathbb{P}^{2}}(1)\right)
$$

and there is a $\mathbb{P}^{1}$-bundle $\pi: V_{7} \rightarrow \mathbb{P}^{2}$. Let $E$ be the exceptional divisor of $\alpha$. Then $E$ is a section of $\pi$. 
There is a quartic surface $R \subset \mathbb{P}^{3}$ such that $\operatorname{Sing}(R)=O$, the point $O$ is an isolated double point of the surface $R$, and there is a commutative diagram

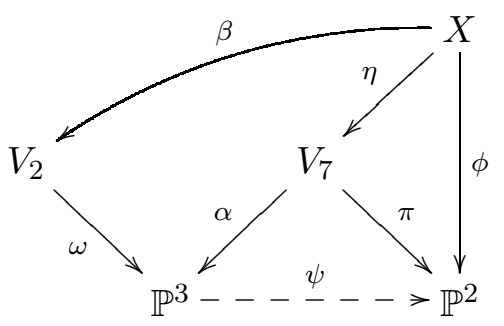

where $\omega$ is a double cover branched in $R$, the morphism $\eta$ is a double cover branched in the proper transform of $R$, the morphism $\beta$ is a birational morphism that contracts a surface $\bar{E}$ such that $\eta(\bar{E})=E$ to the singular point of $V_{2}$ and

$$
\omega\left(\operatorname{Sing}\left(V_{2}\right)\right)=O
$$

the map $\psi$ is a projection from the point $O$, and $\phi$ is a conic bundle.

We assume that $X$ satisfies the following mild generality condition: the point $O$ is an ordinary double point of the surface $R$. Then $\bar{E} \cong \mathbb{P}^{1} \times \mathbb{P}^{1}$.

Let $\bar{H}$ be the proper transform on $X$ of the general plane in $\mathbb{P}^{3}$ that passes through $O$. Then

$$
-K_{X} \sim 2 \bar{H}+\bar{E},
$$

which implies that $\operatorname{lct}(X) \leqslant 1 / 2$.

We suppose that $\operatorname{lct}(X)<1 / 2$. Then there exists an effective $\mathbb{Q}$-divisor $D \sim_{\mathbb{Q}}-K_{X}$ such that the log pair $(X, \lambda D)$ is not log canonical for some positive rational number $\lambda<1 / 2$.

It follows from Lemma 2.18 that $\operatorname{LCS}(X, D) \cap \bar{E} \neq \varnothing$. Put

$$
D=\mu \bar{E}+\Omega,
$$

where $\Omega$ is an effective $\mathbb{Q}$-divisor on $X$ such that $\bar{E} \not \subset \operatorname{Supp}(\Omega)$. Then

$$
2=D \cdot \Gamma=(\mu \bar{E}+\Omega) \cdot \Gamma=2 \mu+\Omega \cdot \Gamma \geqslant 2 \mu,
$$

where $\Gamma$ is a general fiber of the conic bundle $\phi$. Hence $\mu \leqslant 1$. Thus, the log pair

$$
\left(\bar{E},\left.\lambda \Omega\right|_{\bar{E}}\right)
$$

is not $\log$ canonical by Theorem 2.20 , because $\operatorname{LCS}(X, D) \cap \bar{E} \neq \varnothing$. But

$$
\left.\Omega\right|_{\bar{E}} \sim \mathbb{Q}-\frac{1+\mu}{2} K_{\bar{E}}
$$

which is impossible by Lemma 2.24 .

Lemma 8.5. Suppose that $I(X)=2.11$ and $X$ is general. Then $\operatorname{lct}(X)=1 / 2$.

Proof. Let $V$ be a cubic hypersurface in $\mathbb{P}^{4}$. Then there is a commutative diagram

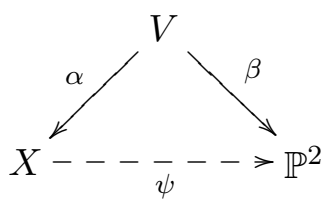

such that $\alpha$ contracts a surface $E \subset X$ to a line $L \subset V$, the map $\psi$ is a linear projection from the line $L$, the morphism $\beta$ is a conic bundle.

We assume that $X$ satisfies the following generality condition: the normal bundle $\mathcal{N}_{L / V}$ to the line $L$ on the variety $V$ is isomorphic to $\mathcal{O}_{L} \oplus \mathcal{O}_{L}$.

Let $H$ be a hyperplane section of $V$ such that $L \subset H$. Then

$$
-K_{X} \sim 2 \bar{H}+E,
$$

where $\bar{H} \subset X$ is the proper transform of the surface $H$. In particular, $\operatorname{lct}(X) \leqslant 1 / 2$. 
We suppose that $\operatorname{lct}(X)<1 / 2$. Then there exists an effective $\mathbb{Q}$-divisor $D \sim_{\mathbb{Q}}-K_{X}$ such that the log pair $(X, \lambda D)$ is not log canonical for some positive rational number $\lambda<1 / 2$. Then

$$
\varnothing \neq \operatorname{LCS}(X, \lambda D) \subseteq E,
$$

since $\operatorname{lct}(V)=1 / 2$ and $\alpha(D) \sim_{\mathbb{Q}}-K_{V}$. Note that $E \cong \mathbb{P}^{1} \times \mathbb{P}^{1}$ by the generality condition.

Let $F \subset E$ be a fiber of the induced projection $E \rightarrow L$, let $Z \subset E$ be a section of this projection such that $Z \cdot Z=0$. Then $\left.\alpha^{*}(H)\right|_{E} \sim F$ and $\left.E\right|_{E} \sim-Z$, because

$$
-2 Z-\left.\left.2 F \sim K_{E} \sim\left(K_{X}+E\right)\right|_{E} \sim 2\left(E-\alpha^{*}(H)\right)\right|_{E} \sim-2 F+\left.2 E\right|_{E} .
$$

Put $D=\mu E+\Omega$, where $\Omega$ is an effective $\mathbb{Q}$-divisor on $X$ such that $E \not \subset \operatorname{Supp}(\Omega)$. Then

$$
2=D \cdot \Gamma=\mu E \cdot \Gamma+\Omega \cdot \Gamma \geqslant \mu E \cdot \Gamma=2 \mu,
$$

where $\Gamma$ is a general fiber of the conic bundle $\beta$. Thus, we see that $\mu \leqslant 1$.

The log pair $\left(E,\left.\lambda \Omega\right|_{E}\right)$ is not log canonical by Theorem 2.20, But

$$
\left.\left.\Omega\right|_{E} \sim_{\mathbb{Q}}\left(-K_{X}-\mu E\right)\right|_{E} \sim_{\mathbb{Q}}(1+\mu) Z+2 F,
$$

which contradicts Lemma 2.24 , because $\mu \leqslant 1$ and $\lambda<1 / 2$.

Lemma 8.6. Suppose that $I(X)=2.15$ and $X$ is general. Then $\operatorname{lct}(X)=1 / 2$.

Proof. There is a birational morphism $\alpha: X \rightarrow \mathbb{P}^{3}$ that contracts a surface $E \subset X$ to a smooth curve $C \subset \mathbb{P}^{3}$ that is complete intersection of an irreducible quadric $Q \subset \mathbb{P}^{3}$ and a cubic $F \subset \mathbb{P}^{3}$.

We assume that $X$ satisfies the following generality condition: the quadric $Q$ is smooth.

Let $\bar{Q}$ be a proper transform of $Q$ on the threefold $X$. Then there is a commutative diagram

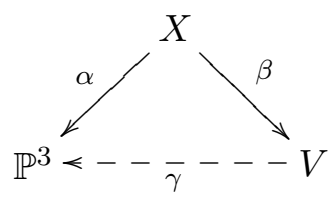

where $V$ is a cubic in $\mathbb{P}^{4}$ that has one ordinary double point $P \in V$, the morphism $\beta$ contracts the surface $\bar{Q}$ to the point $P$, and $\gamma$ is a linear projection from the point $P$.

Let $E$ be the exceptional divisor of $\alpha$. Then

$$
-K_{X} \sim 2 \bar{Q}+E
$$

and $\beta(E) \subset V$ is a surface that contains all lines on $V$ that pass through $P$. We see that $\operatorname{lct}(X) \leqslant 1 / 2$.

We suppose that $\operatorname{lct}(X)<1 / 2$. Then there exists an effective $\mathbb{Q}$-divisor $D \sim_{\mathbb{Q}}-K_{X}$ such that the log pair $(X, \lambda D)$ is not log canonical for some positive rational number $\lambda<1 / 2$.

It follows from Lemma 2.17 that either

$$
\varnothing \neq \operatorname{LCS}(X, \lambda D) \subseteq \bar{Q},
$$

or the set $\operatorname{LCS}(X, \lambda D)$ contains a fiber of the natural projection $E \rightarrow C$. In both cases

$$
\operatorname{LCS}(X, \lambda D) \cap \bar{Q} \neq \varnothing .
$$

We have $\bar{Q} \cong \mathbb{P}^{1} \times \mathbb{P}^{1}$. Put

$$
D=\mu \bar{Q}+\Omega,
$$

where $\Omega$ is an effective $\mathbb{Q}$-divisor on $X$ such that $\bar{Q} \not \subset \operatorname{Supp}(\Omega)$. Then

$$
\alpha(D) \sim_{\mathbb{Q}} \mu Q+\alpha(\Omega) \sim_{\mathbb{Q}}-K_{\mathbb{P}^{3}},
$$

which gives $\mu \leqslant 2$. The $\log$ pair $\left(\bar{Q},\left.\lambda \Omega\right|_{\bar{Q}}\right)$ is not $\log$ canonical by Theorem 2.20 , But

$$
\left.\Omega\right|_{\bar{Q}} \sim \mathbb{Q}-\frac{1+\mu}{2} K_{\bar{Q}},
$$

which implies that $\mu>1$ by Lemma 2.24 , 
It follows from Remark 2.23 that we may assume that $E \not \subset \operatorname{Supp}(D)$. Then

$$
1=D \cdot F=\mu \bar{Q} \cdot F+\Omega \cdot F=\mu+\Omega \cdot F \geqslant \mu,
$$

where $F$ is a general fiber the natural projection $E \rightarrow C$. But $\mu>1$, which is a contradiction.

Lemma 8.7. Suppose that $I(X)=2.18$. Then $\operatorname{lct}(X)=1 / 2$.

Proof. There is a smooth divisor $B \subset \mathbb{P}^{1} \times \mathbb{P}^{2}$ of bidegree $(2,2)$ such that the diagram

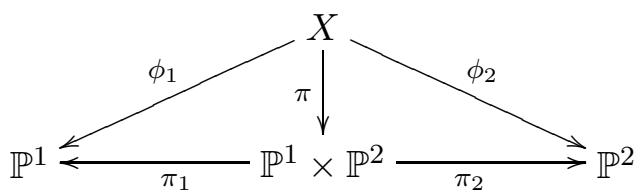

commutes, where $\pi$ is a double cover that is branched in $B$, the morphisms $\pi_{1}$ and $\pi_{2}$ are natural projections, the morphism $\phi_{1}$ is a quadric fibration, and $\phi_{2}$ is a conic bundle.

Let $H_{1}$ be a general fiber of $\pi_{1}$, and let $H_{2}$ be a general surface in $\left|\pi_{2}^{*}\left(\mathcal{O}_{\mathbb{P}^{2}}(1)\right)\right|$. Then $B \sim 2 H_{1}+2 H_{2}$.

Let $\bar{H}_{1}$ be a general fiber of $\phi_{1}$, and let $\bar{H}_{2}$ be a general surface in $\left|\phi_{2}^{*}\left(\mathcal{O}_{\mathbb{P}^{2}}(1)\right)\right|$. Then

$$
-K_{X} \sim \bar{H}_{1}+2 \bar{H}_{2}
$$

which implies that $\operatorname{lct}(X) \leqslant 1 / 2$.

We suppose that $\operatorname{lct}(X)<1 / 2$. Then there exists an effective $\mathbb{Q}$-divisor $D \sim_{\mathbb{Q}}-K_{X}$ such that the log pair $(X, \lambda D)$ is not log canonical for some positive rational number $\lambda<1 / 2$.

Applying Lemma 2.26 to the fibration $\phi_{1}$ we see that

$$
\varnothing \neq \operatorname{LCS}(X, \lambda D) \subseteq Q,
$$

where $Q$ is a singular fiber of $\phi_{1}$. Moreover, applying Theorem 2.28 to the fibration $\phi_{2}$, we see that

$$
\varnothing \neq \operatorname{LCS}(X, \lambda D) \subseteq Q \cap R
$$

where $R \subset X$ be an irreducible surface that is swept out by singular fibers of $\phi_{2}$. In particular, the set $\mathbb{L} \mathbb{C} \mathbb{S}(X, \lambda D)$ contains no surfaces.

Suppose that $\operatorname{LCS}(X, \lambda D)$ is zero-dimensional. Then

$$
\operatorname{LCS}\left(X, \lambda D+\frac{1}{2}\left(\bar{H}_{1}+2 \bar{H}_{2}\right)\right)=\operatorname{LCS}(X, \lambda D) \cup \bar{H}_{2},
$$

which is impossible by Theorem 2.7 ,

We see that the set $\mathbb{L} \mathbb{C} S(X, \lambda D)$ contains a curve $\Gamma \subset Q \cap R$. Put

$$
D=\mu Q+\Omega,
$$

where $\Omega$ is an effective $\mathbb{Q}$-divisor such that $Q \not \subset \operatorname{Supp}(\Omega)$. Then

$$
\left(Q,\left.\lambda \Omega\right|_{Q}\right)
$$

is not $\log$ canonical along $\Gamma$ by Theorem 2.20 , But

$$
\left.\left.\Omega\right|_{Q} \sim_{\mathbb{Q}}\left(-K_{X}-\mu Q\right)\right|_{Q} \sim_{\mathbb{Q}}-K_{Q}
$$

which implies that $\Gamma$ is a ruling of the cone $Q \subset \mathbb{P}^{3}$ by Lemma 5.7. Then $\phi_{2}(\Gamma) \subset \mathbb{P}^{2}$ is a line, and

$$
\phi_{2}(\Gamma) \subseteq \phi_{2}(R) .
$$

But $\phi_{2}(R) \subset \mathbb{P}^{2}$ is a curve of degree 4 . Thus, we see that

$$
\phi_{2}(R)=\phi_{2}(\Gamma) \cup Z,
$$

where $Z \subset \mathbb{P}^{2}$ is a reduced cubic curve. Then $\phi_{2}$ induces a double cover of

$$
\phi_{2}(\Gamma) \backslash \underset{55}{\left(\phi_{2}(\Gamma) \cap Z\right)}
$$


that must be unramified (see [192]). But the quartic curve $\phi_{2}(R)$ has at most ordinary double points (see [192], [166]). Then

which is impossible, because $\phi_{2}(\Gamma) \cong \mathbb{P}^{1}$.

$$
\left|\phi_{2}(\Gamma) \cap Z\right|=3,
$$

Lemma 8.8. Suppose that $I(X)=2.19$ and $X$ is general. Then $\operatorname{lct}(X)=1 / 2$.

Proof. It follows from [98, Proposition 3.4.1] that there is a commutative diagram

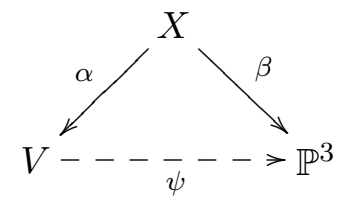

where $V$ is a complete intersection of two quadric fourfolds in $\mathbb{P}^{5}$, the morphism $\alpha$ is a blow up of a line $L \subset V$, the morphism $\beta$ is a blow up of a smooth curve $C \subset \mathbb{P}^{3}$ of degree 5 and genus 2 , and the map $\psi$ is a linear projection from the line $L$.

Let $E$ and $R$ be the exceptional divisors of $\alpha$ and $\beta$, respectively. Then

- the surface $\beta(E) \subset \mathbb{P}^{3}$ is an irreducible quadric,

- the surface $\alpha(R) \subset V$ is swept out by lines that intersect the line $L$.

We assume that $X$ satisfies the following generality condition: the surface $\beta(E)$ is smooth.

Let $H$ be any hyperplane section of $V \subset \mathbb{P}^{5}$ such that $L \subset H$. Then

$$
2 \bar{H}+E \sim R+2 E \sim-K_{X},
$$

where $\bar{H}$ is a proper transform of $H$ on the threefold $X$. We see that $\operatorname{lct}(X) \leqslant 1 / 2$.

We suppose that $\operatorname{lct}(X)<1 / 2$. Then there exists an effective $\mathbb{Q}$-divisor $D \sim_{\mathbb{Q}}-K_{X}$ such that the log pair $(X, \lambda D)$ is not log canonical for some positive rational number $\lambda<1 / 2$. Then

$$
\varnothing \neq \operatorname{LCS}(X, \lambda D) \subseteq E \cong \mathbb{P}^{1} \times \mathbb{P}^{1},
$$

because $\alpha(D) \sim_{\mathbb{Q}}-K_{V}$ and $\operatorname{lct}(V)=1 / 2$ by Theorem 7.1 .

Let $F$ be a fiber of the projection $E \rightarrow L$, and let $Z$ be a section of this projection such that $Z \cdot Z=0$. Then $\left.\alpha^{*}(H)\right|_{E} \sim F$ and $\left.E\right|_{E} \sim-Z$, because

$$
-2 Z-\left.\left.\left.2 F \sim K_{E} \sim\left(K_{X}+E\right)\right|_{E} \sim 2\left(E-\alpha^{*}(H)\right)\right|_{E} \sim 2 E\right|_{E}-2 F .
$$

By Remark 2.23, we may assume that either $E \not \subset \operatorname{Supp}(D)$, or $R \not \subset \operatorname{Supp}(D)$, because the log pair

$$
(X, \lambda(R+2 E))
$$

is $\log$ canonical and $-K_{X} \sim R+2 E$. Put

$$
D=\mu E+\Omega,
$$

where $\Omega$ is an effective $\mathbb{Q}$-divisor on $X$ such that $E \not \subset \operatorname{Supp}(\Omega)$.

Suppose that $\mu \leqslant 1$. Then $(X, E+\lambda \Omega)$ is not $\log$ canonical, which implies that

$$
\left(E,\left.\lambda \Omega\right|_{E}\right)
$$

is also not $\log$ canonical by Theorem 2.20 , But

$$
\left.\left.\Omega\right|_{E} \sim_{\mathbb{Q}}\left(-K_{X}-\mu E\right)\right|_{E} \sim_{\mathbb{Q}}(1+\mu) Z+2 F,
$$

which contradicts Lemma 2.24 , because $\mu \leqslant 1$ and $\lambda<1 / 2$.

Thus, we see that $\mu>1$. Then we may assume that $R \not \subset \operatorname{Supp}(D)$.

Let $\Gamma$ be a general fiber of the projection $R \rightarrow C$. Then $\Gamma \not \subset \operatorname{Supp}(D)$ and

$$
1=-K_{X} \cdot \Gamma=\mu E \cdot \Gamma+\Omega \cdot \Gamma=\mu+\Omega \cdot \Gamma \geqslant \mu,
$$

which is a contradiction.

Lemma 8.9. Suppose that $I(X)=2.23$ and $X$ is general. Then $\operatorname{lct}(X)=1 / 3$. 
Proof. There is a birational morphism $\alpha: X \rightarrow Q$ such that $Q \subset \mathbb{P}^{4}$ is a smooth quadric threefold, and $\alpha$ contracts a surface $E \subset X$ to a smooth curve $C \subset Q$ that is a complete intersection of a hyperplane section $H \subset Q$ and a divisor $F \in\left|\mathcal{O}_{Q}(2)\right|$.

We assume that $X$ satisfies the following generality condition: the quadric surface $H$ is smooth.

Let $\bar{H}$ be a proper transform of $H$ on the threefold $X$. Then there is a commutative diagram

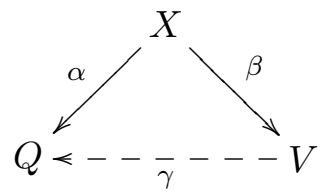

where $V$ is a complete intersection of two quadrics in $\mathbb{P}^{5}$ such that $V$ has one ordinary double point $P \in V$, the morphism $\beta$ contracts $\bar{H}$ to the point $P$, and $\gamma$ is a projection from $P$.

Let $E$ be the exceptional divisor of $\alpha$. Then

$$
-K_{X} \sim 3 \bar{H}+2 E
$$

and $\beta(E) \subset V$ is a surface that contains all lines that pass through $P$. In particular, $\operatorname{lct}(X) \leqslant$ $1 / 3$.

We suppose that $\operatorname{lct}(X)<1 / 3$. Then there exists an effective $\mathbb{Q}$-divisor $D \sim_{\mathbb{Q}}-K_{X}$ such that the log pair $(X, \lambda D)$ is not log canonical for some positive rational number $\lambda<1 / 3$.

It follows from Remark 7.8 that either

$$
\varnothing \neq \operatorname{LCS}(X, \lambda D) \subseteq \bar{H},
$$

or the set $\operatorname{LCS}(X, \lambda D)$ contains a fiber of the natural projection $E \rightarrow C$. In both cases

$$
\operatorname{LCS}(X, \lambda D) \cap \bar{H} \neq \varnothing .
$$

We have $\bar{H} \cong \mathbb{P}^{1} \times \mathbb{P}^{1}$. There is a non-negative rational number $\mu$ such that

$$
D=\mu \bar{H}+\Omega,
$$

where $\Omega$ is an effective $\mathbb{Q}$-divisor on $X$ such that $\bar{H} \not \subset \operatorname{Supp}(\Omega)$. Then

$$
\alpha(D) \sim_{\mathbb{Q}} \mu H+\alpha(\Omega) \sim_{\mathbb{Q}}-K_{Q},
$$

which gives $\mu \leqslant 3$. The log pair $\left(\bar{H},\left.\lambda \Omega\right|_{\bar{H}}\right)$ is not log canonical by Theorem 2.20 , But

$$
\left.\Omega\right|_{\bar{H}} \sim \mathbb{Q}-\frac{1+\mu}{2} K_{\bar{H}}
$$

which implies that $\mu>1$ by Lemma 2.24 .

It follows from Remark 2.23 that we may assume that $E \not \subset \operatorname{Supp}(D)$, because the log pair

$$
(X, \lambda(3 \bar{H}+2 E))
$$

is $\log$ canonical. Let $F$ be a general fiber the natural projection $E \rightarrow C$. Then

$$
1=D \cdot F=\mu \bar{H} \cdot F+\Omega \cdot F=\mu+\Omega \cdot F \geqslant \mu,
$$

which is a contradiction, because $\mu>1$.

Lemma 8.10. Suppose that $I(X)=2.24$ and $X$ is general. Then $\operatorname{lct}(X)=1 / 2$.

Proof. The threefold $X$ is a divisor on $\mathbb{P}^{2} \times \mathbb{P}^{2}$ of bidegree $(1,2)$. Let $H_{i}$ be a surface in

$$
\left|\pi_{i}^{*}\left(\mathcal{O}_{\mathbb{P}^{2}}(1)\right)\right|,
$$

where $\pi_{i}: X \rightarrow \mathbb{P}^{2}$ is a projection of $X$ onto the $i$-th factor of $\mathbb{P}^{2} \times \mathbb{P}^{2}, i \in\{1,2\}$. Then

$$
-K_{X} \sim 2 H_{1}+H_{2}
$$

which implies that $\operatorname{lct}(X) \leqslant 1 / 2$. Note that $\pi_{1}$ is a conic bundle, and $\pi_{2}$ is a $\mathbb{P}^{1}$-bundle.

Let $\Delta \subset \mathbb{P}^{2}$ be the degeneration curve of the conic bundle $\pi_{1}$. Then $\Delta$ is a cubic curve.

We suppose that $X$ satisfies the following generality condition: the curve $\Delta$ is irreducible. 
Assume that $\operatorname{lct}(X)<1 / 2$. Then there exists an effective $\mathbb{Q}$-divisor $D \sim_{\mathbb{Q}}-K_{X}$ such that the log pair $(X, \lambda D)$ is not log canonical for some positive rational number $\lambda<1 / 2$.

Suppose that the set $\mathbb{L} \mathbb{C} S(X, \lambda D)$ contains a surface $S \subset X$. Then

$$
D=\mu S+\Omega,
$$

where $\Omega$ is an effective $\mathbb{Q}$-divisor such that $S \not \subset \operatorname{Supp}(\Omega)$, and $\mu>1 / \lambda$. Let $F_{i}$ be a general fiber of $\pi_{i}, i \in\{1,2\}$. Then

$$
2=D \cdot F_{i}=\mu S \cdot F_{i}+\Omega \cdot F_{i} \geqslant \mu S \cdot F_{i},
$$

but either $S \cdot F_{1} \geqslant 1$ or $S \cdot F_{2} \geqslant 1$. Thus, we see that $\mu \leqslant 2$, which is a contradiction.

By Theorem 2.28 and Theorem 2.7, there is a fiber $\Gamma_{2}$ of the $\mathbb{P}^{1}$-bundle $\pi_{2}$ such that

$$
\varnothing \neq \operatorname{LCS}(X, \lambda D)=\Gamma_{2},
$$

because the set $\mathbb{L} \mathbb{C} S(X, \lambda D)$ contains no surfaces.

Applying Theorem 2.28 to the conic bundle $\pi_{1}$, we see that

$$
\pi_{1}\left(\Gamma_{2}\right) \subset \Delta,
$$

which is impossible, because $\Delta \subset \mathbb{P}^{2}$ is an irreducible cubic curve and $\pi_{1}\left(\Gamma_{2}\right) \subset \mathbb{P}^{2}$ is a line.

Lemma 8.11. Suppose that $I(X)=2.25$. Then $\operatorname{lct}(X)=1 / 2$.

Proof. Recall that $X$ is a blow up $\alpha: X \rightarrow \mathbb{P}^{3}$ along a normal elliptic curve $C$ of degree 4 .

Let $Q \subset \mathbb{P}^{3}$ be a general quadric containing $C$, and let $\bar{Q} \subset X$ be a proper transform of $Q$. Then

$$
-K_{X} \sim 2 \bar{Q}+E,
$$

where $E$ is the exceptional divisor of $\alpha$. In particular, $\operatorname{lct}(X) \leqslant 1 / 2$.

We suppose that $\operatorname{lct}(X)<1 / 2$. Then there exists an effective $\mathbb{Q}$-divisor $D \sim_{\mathbb{Q}}-K_{X}$ such that the log pair $(X, \lambda D)$ is not log canonical for some positive rational number $\lambda<1 / 2$.

Note that the linear system $|\bar{Q}|$ defines a quadric fibration

$$
\phi: X \longrightarrow \mathbb{P}^{1},
$$

such that every fiber of $\phi$ is irreducible. Then the log pair $(X, \lambda D)$ is log canonical along every nonsingular fiber $\tilde{Q}$ of the fibration $\phi$ by Theorem 2.28 since $\operatorname{lct}(\tilde{Q})=1 / 2$ (see Example 1.18).

The locus $\operatorname{LCS}(X, \lambda D)$ does not contain any fiber of $\phi$, because $\alpha(D) \sim_{\mathbb{Q}} 2 Q$ and every fiber of $\phi$ is irreducible. Therefore, we see that $\operatorname{dim}(\operatorname{LCS}(X, \lambda D)) \leqslant 1$.

Let $Z$ be an element in $\mathbb{L} \mathbb{C S}(X, \lambda D)$. There is a singular fiber $\bar{Q}_{1}$ of the fibration $\phi$ such that $Z \subset \bar{Q}_{1}$. Note that $\phi$ has 4 singular fibers and each of them is a proper transform of a quadric cone in $\mathbb{P}^{3}$ with vertex outside $C$.

Let $\bar{Q}_{2}$ be a singular fiber of $\phi$ such that $\bar{Q}_{1} \neq \bar{Q}_{2}$, let $\bar{H}$ be a proper transform of a general plane in $\mathbb{P}^{3}$ that is tangent to the cone $\alpha\left(\bar{Q}_{2}\right) \subset \mathbb{P}^{3}$ along one of its rulings $L \subset \alpha\left(\bar{Q}_{2}\right)$, and let $\bar{R}$ be a proper transform of a very general plane in $\mathbb{P}^{3}$. Put

$$
\Delta=\lambda D+\frac{1}{2}\left((1+\varepsilon) \bar{Q}_{2}+(2-\varepsilon) \bar{H}+3 \varepsilon R\right)
$$

for some positive rational number $\varepsilon<1-2 \lambda$. Then

$$
\Delta \sim_{\mathbb{Q}}-\left(\lambda+\frac{1}{2}(1+\varepsilon)\right) K_{X} \sim_{\mathbb{Q}}-\frac{1+\varepsilon+2 \lambda}{2} K_{X},
$$

which implies that $-\left(K_{X}+\Delta\right)$ is ample.

Let $\bar{L}$ be a proper transform on $X$ of the line $L$. Then

$$
Z \cup \bar{L} \subset \operatorname{LCS}(X, \Delta) \subset \bar{Q}_{1} \cup \bar{Q}_{2},
$$

which is impossible by Theorem 2.7 , because $-\left(K_{X}+\Delta\right)$ is ample.

Lemma 8.12. Suppose that $I(X)=2.26$ and $X$ is general. Then $\operatorname{lct}(X)=1 / 2$. 
Proof. Let $V$ be a smooth Fano threefold such that $-K_{V} \sim 2 H$ and

$$
\operatorname{Pic}(V)=\mathbb{Z}[H],
$$

where $H$ is a Cartier divisor such that $H^{3}=5$ (i.e. $I(V)=1.15$ ). Then $|H|$ induces an embedding $X \subset \mathbb{P}^{6}$.

It follows from [98, Proposition 3.4.1] (see also [67]) that there is a line

$$
L \subset X \subset \mathbb{P}^{6}
$$

such that there is a commutative diagram

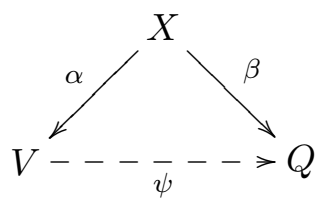

where $Q$ is a smooth quadric hypersurface in $\mathbb{P}^{4}$, the morphism $\alpha$ is a blow up of the line $L \subset V$, the morphism $\beta$ is a blow up of a twisted cubic curve $\mathbb{P}^{1} \cong C \subset Q$, and $\psi$ is a projection from the line $L$.

Let $S$ be the exceptional divisor of the morphism $\beta$. Put $\bar{S}=\alpha(S)$. Then $\bar{S} \sim H$, and $\bar{S}$ is singular along the line $L$. Let $E$ be the exceptional divisor of the morphism $\alpha$. Then

$$
\left.\beta(E) \sim \mathcal{O}_{\mathbb{P}^{4}}(1)\right|_{Q}
$$

which implies that $\beta(E)$ is an irreducible quadric surface.

We suppose that $X$ satisfies the following generality condition: the surface $\beta(E)$ is smooth.

The equivalence $-K_{X} \sim 2 S+3 E$ holds. Moreover, the log pair

$$
\left(X, \frac{1}{3}(2 S+3 E)\right)
$$

is $\log$ canonical but not log terminal. Thus, we see that $\operatorname{lct}(X) \leqslant 1 / 3$.

We suppose that $\operatorname{lct}(X)<1 / 3$. Then there exists an effective $\mathbb{Q}$-divisor $D \sim_{\mathbb{Q}}-K_{X}$ such that the $\log$ pair $(X, \lambda D)$ is not log canonical for some positive rational number $\lambda<1 / 3$. Then

$$
\varnothing \neq \operatorname{LCS}(X, \lambda D) \subseteq E,
$$

because $\alpha(D) \sim_{\mathbb{Q}}-K_{V}$ and $\operatorname{lct}(V)=1 / 2$ by Theorem [7.1.

Note that $E \cong \mathbb{P}^{1} \times \mathbb{P}^{1}$ by our generality condition. Let $F$ be a fiber of the projection $E \rightarrow L$, and let $Z$ be a section of this projection such that $Z \cdot Z=0$. Then $\left.\alpha^{*}(H)\right|_{E} \sim F$ and $\left.E\right|_{E} \sim-Z$, because

$$
-2 Z-\left.\left.\left.2 F \sim K_{E} \sim\left(K_{X}+E\right)\right|_{E} \sim 2\left(E-\alpha^{*}(H)\right)\right|_{E} \sim 2 E\right|_{E}-2 F .
$$

By Remark 2.23, we may assume that either $E \not \subset \operatorname{Supp}(D)$, or $S \not \subset \operatorname{Supp}(D)$. Put

$$
D=\mu E+\Omega,
$$

where $\Omega$ is an effective $\mathbb{Q}$-divisor on $X$ such that $E \not \subset \operatorname{Supp}(\Omega)$.

Suppose that $\mu \leqslant 2$. Then $(X, E+\lambda \Omega)$ is not log canonical, which implies that

$$
\left(E,\left.\lambda \Omega\right|_{E}\right)
$$

is also not log canonical by Theorem 2.20, But

$$
\left.\left.\Omega\right|_{E} \sim_{\mathbb{Q}}\left(-K_{X}-\mu E\right)\right|_{E} \sim_{\mathbb{Q}}(1+\mu) Z+2 F,
$$

which contradicts Lemma 2.24 , because $\mu \leqslant 2$ and $\lambda<1 / 3$.

Thus, we see that $\mu>2$. Then we may assume that $S \not \subset \operatorname{Supp}(D)$.

Let $\Gamma$ be a general fiber of the projection $S \rightarrow C$. Then $\Gamma \not \subset \operatorname{Supp}(D)$ and

$$
1=-K_{X} \cdot \Gamma=\mu E \cdot \Gamma+\Omega \cdot \Gamma=\mu+\Omega \cdot \Gamma \geqslant \mu,
$$

which is a contradiction.

Lemma 8.13. Suppose that $I(X)=2.27$. Then $\operatorname{lct}(X)=1 / 2$. 
Proof. There is a morphism $\alpha: X \rightarrow \mathbb{P}^{3}$ contracting a surface $E$ to a twisted cubic curve $C \subset \mathbb{P}^{3}$, and $X \cong \mathbb{P}(\mathcal{E})$, where $\mathcal{E}$ is a stable rank two vector bundle on $\mathbb{P}^{2}$ with $c_{1}(\mathcal{E})=0$ and $c_{1}(\mathcal{E})=2$ such that the sequence

$$
0 \longrightarrow \mathcal{O}_{\mathbb{P}_{2}}(-1) \oplus \mathcal{O}_{\mathbb{P}_{2}}(-1) \longrightarrow \mathcal{O}_{\mathbb{P}_{2}} \oplus \mathcal{O}_{\mathbb{P}_{2}} \oplus \mathcal{O}_{\mathbb{P}_{2}} \oplus \mathcal{O}_{\mathbb{P}_{2}} \longrightarrow \mathcal{E} \otimes \mathcal{O}_{\mathbb{P}_{2}}(1) \longrightarrow 0
$$

is exact (see [50] and [177, Application 1]).

Let $Q \subset \mathbb{P}^{3}$ be a general quadric containing $C$, and let $\bar{Q} \subset X$ be a proper transform of $Q$. Then

$$
-K_{X} \sim 2 \bar{Q}+E,
$$

where $E$ is the exceptional divisor of $\alpha$. In particular, we see that $\operatorname{lct}(X) \leqslant 1 / 2$.

We suppose that $\operatorname{lct}(X)<1 / 2$. Then there exists an effective $\mathbb{Q}$-divisor $D \sim_{\mathbb{Q}}-K_{X}$ such that the log pair $(X, \lambda D)$ is not log canonical for some positive rational number $\lambda<1 / 2$.

Suppose that the set $\mathbb{L} \mathbb{C S}(X, \lambda D)$ contains a surface $S \subset X$. Put

$$
D=\mu F+\Omega,
$$

where $\mu \geqslant 1 / \lambda$ and $\Omega$ is an effective $\mathbb{Q}$-divisor such that $F \not \subset \operatorname{Supp}(\Omega)$.

Let $\phi: X \rightarrow \mathbb{P}^{2}$ be the natural $\mathbb{P}^{1}$-bundle. Then

$$
2=D \cdot \Gamma=\mu F \cdot \Gamma+\Omega \cdot \Gamma=\mu F \cdot \Gamma+\Omega \cdot F \geqslant \mu F \cdot \Gamma,
$$

where $\Gamma$ is a general fiber of $\phi$. Thus, we see that $F$ is swept out by the fibers of $\phi$. Then

$$
\alpha(F) \sim \mathcal{O}_{\mathbb{P}^{3}}(d)
$$

and $d \geqslant 2$. But $\alpha(D) \sim_{\mathbb{Q}} \mu \alpha(F)+\alpha(\Omega) \sim_{\mathbb{Q}} \mathcal{O}_{\mathbb{P}^{3}}(4)$, which is a contradiction.

We wee that the locus $\operatorname{LCS}(X, \lambda D)$ contains no surfaces. Applying Theorem 2.28 to $(X, \lambda D)$ and $\phi$, we see that

$$
L \subseteq \operatorname{LCS}(X, \lambda D)
$$

where $L$ is a fiber of $\phi$. Note that $\alpha(L)$ is a secant line of the curve $C \subset \mathbb{P}^{3}$. One has

$$
\alpha(L) \subseteq \operatorname{LCS}\left(\mathbb{P}^{3}, \lambda \alpha(D)\right) \subseteq \alpha(\operatorname{LCS}(X, \lambda D)) \cup C,
$$

which is impossible by Lemma 2.9.

Lemma 8.14. Suppose that $I(X)=2.28$. Then $\operatorname{lct}(X)=1 / 4$.

Proof. There is a blow up $\alpha: X \rightarrow \mathbb{P}^{3}$ along a plane cubic curve $C \subset \mathbb{P}^{3}$. One has

$$
-K_{X} \sim 4 G+3 E
$$

where $E$ is the exceptional divisor of $\alpha$ and $G$ is a proper transform of the plane in $\mathbb{P}^{3}$ that contains the curve $C$. In particular, we see that the inequality $\operatorname{lct}(X) \leqslant 1 / 4$ holds.

We suppose that $\operatorname{lct}(X)<1 / 4$. Then there exists an effective $\mathbb{Q}$-divisor $D \sim_{\mathbb{Q}}-K_{X}$ such that the $\log$ pair $(X, \lambda D)$ is not $\log$ canonical for some rational number $\lambda<1 / 4$. One has

$$
\varnothing \neq \operatorname{LCS}(X, \lambda D) \subseteq E,
$$

since $\operatorname{lct}\left(\mathbb{P}^{4}\right)=1 / 4$. Computing the intersections with a strict transform of a general line in $\mathbb{P}^{3}$ intersecting the curve $C$, one obtains that $\operatorname{LCS}(X, \lambda D)$ does not contain the divisor $E$. Moreover, any curve $\Gamma \in \mathbb{L} \mathbb{C S}(X, \lambda D)$ must be a fiber of the natural projection

$$
\psi: E \longrightarrow C
$$

by Lemma 2.14. Therefore, we see that either the locus $\operatorname{LCS}(X, \lambda D)$ consists of a single point, or the locus $\operatorname{LCS}(X, \lambda D)$ consists of a single fiber of the projection $\psi$ by Theorem 2.7 .

Let $R$ be a sufficiently general cone in $\mathbb{P}^{3}$ over the curve $C$, and let $H$ be a sufficiently general plane in $\mathbb{P}^{3}$ that passes through the point Sing $(R)$. Then

$$
\operatorname{LCS}\left(X, \lambda D+\frac{3}{4}(\bar{R}+\bar{H})\right)=\operatorname{LCS}(X, \lambda D) \bigcup \operatorname{Sing}(\bar{R}),
$$


where $\bar{R}$ and $\bar{H}$ are proper transforms of $R$ and $H$ on the threefold $X$. But the divisor

$$
-\left(K_{X}+\lambda D+\frac{3}{4}(\bar{R}+\bar{H})\right) \sim_{\mathbb{Q}}(\lambda-1 / 4) K_{X}
$$

is ample, which contradicts Theorem 2.7.

Lemma 8.15. Suppose that $I(X)=2.29$. Then $\operatorname{lct}(X)=1 / 3$.

Proof. There is a birational morphism $\alpha: X \rightarrow Q$ such that $Q$ is a smooth quadric hypersurface, and $\alpha$ is a blow up along a smooth conic $C \subset Q$.

Let $H$ be a general hyperplane section of $Q \subset \mathbb{P}^{4}$ that contains $C$, and let $\bar{H}$ be a proper transform of the surface $H$ on the threefold $X$. Then

$$
-K_{X} \sim 3 \bar{H}+2 E
$$

where $E$ is the exceptional divisor of $\alpha$. In particular, the inequality lct $(X) \leqslant 1 / 3$ holds.

We suppose that $\operatorname{lct}(X)<1 / 3$. Then there exists an effective $\mathbb{Q}$-divisor $D \sim_{\mathbb{Q}}-K_{X}$ such that the log pair $(X, \lambda D)$ is not $\log$ canonical for some rational $\lambda<1 / 3$. Then

$$
\varnothing \neq \operatorname{LCS}(X, \lambda D) \subseteq E
$$

since $\operatorname{lct}(Q)=1 / 3($ see Example 1.9$)$ and $\alpha(D) \sim_{\mathbb{Q}}-K_{Q}$.

The linear system $|\bar{H}|$ has no base points and defines a morphism $\beta: X \rightarrow \mathbb{P}^{1}$, whose general fiber is a smooth quadric surface. Then the $\log$ pair $(X, \lambda D)$ is $\log$ canonical along the smooth fibers of $\beta$ by Theorem 2.28 (see Example 1.18).

It follows from Theorem 2.7 that there is a singular fiber $S \sim \bar{H}$ of the morphism $\beta$ such that

$$
\varnothing \neq \operatorname{LCS}(X, \lambda D) \subseteq E \cap S
$$

and $\alpha(S) \subset \mathbb{P}^{3}$ is a quadratic cone. Put $\Gamma=E \cap S$. Then $\Gamma$ is an irreducible conic, the log pair

$$
\left(X, S+\frac{2}{3} E\right)
$$

has $\log$ canonical singularities, and $3 S+2 E \sim_{\mathbb{Q}} D$. Therefore, it follows from Remark 2.23 that to complete the proof we may assume that either $S \not \subset \operatorname{Supp}(D)$ or $E \not \subset \operatorname{Supp}(D)$.

Intersecting the divisor $D$ with a strict transform of a general ruling of the cone $\alpha(S) \subset \mathbb{P}^{3}$ and with a general fiber of the projection $E \rightarrow C$, we see that

$$
\Gamma \nsubseteq \operatorname{LCS}(X, \lambda D)
$$

which implies that $\operatorname{LCS}(X, \lambda D)$ consists of a single point $O \in \Gamma$ by Theorem 2.7 .

Let $R$ be a general (not passing through $O$ ) surface in $\left|\alpha^{*}(H)\right|$. Then

$$
\operatorname{LCS}\left(X, \lambda D+\frac{1}{2}(\bar{H}+2 R)\right)=R \cup O,
$$

which is impossible by Theorem 2.7, since $-K_{X} \sim \bar{H}+2 R \sim_{\mathbb{Q}} D$ and $\lambda<1 / 3$.

Lemma 8.16. Suppose that $I(X)=2.30$. Then $\operatorname{lct}(X)=1 / 4$.

Proof. There is a commutative diagram

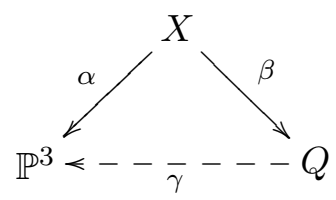

where $Q$ is a smooth quadric threefold in $\mathbb{P}^{4}$, the morphism $\alpha$ is a blow up of a smooth conic $C \subset \mathbb{P}^{3}$, the morphism $\beta$ is a blow up of a point, and $\gamma$ is a projection from a point.

Let $G$ be a proper transform on the variety $X$ of the unique plane in $\mathbb{P}^{3}$ that contains the conic $C$. Then the surface $G$ is contracted by the morphism $\beta$, and

$$
-K_{X} \sim 4 G+3 E
$$

where $E$ is the exceptional divisor of the blow up $\alpha$. Thus, we see that $\operatorname{lct}(X) \leqslant 1 / 4$. 
We suppose that $\operatorname{lct}(X)<1 / 4$. Then there exists an effective $\mathbb{Q}$-divisor $D \sim_{\mathbb{Q}}-K_{X}$ such that the log pair $(X, \lambda D)$ is not log canonical for some rational $\lambda<1 / 4$. Then

$$
\varnothing \neq \operatorname{LCS}(X, \lambda D) \subseteq E \cap G,
$$

because $\operatorname{lct}\left(\mathbb{P}^{4}\right)=1 / 4$ and $\operatorname{lct}(Q)=1 / 3$.

We may assume that either $G \not \subset \operatorname{Supp}(X)$ or $E \not \subset \operatorname{Supp}(X)$ by Remark 2.23.

Intersecting $D$ with lines in $G \cong \mathbb{P}^{2}$ and with fibers of the projection $E \rightarrow C$, we see that

$$
\operatorname{LCS}(X, \lambda D) \subsetneq E \cap G
$$

which implies that there is a point $O \in E \cap G$ such that $\operatorname{LCS}(X, \lambda D)=O$ by Theorem 2.7.

Let $R$ be a general surface in $\left|\alpha^{*}(H)\right|$ and $F$ a general surface in $\left|\alpha^{*}(2 H)-E\right|$. Then

$$
\operatorname{LCS}\left(X, \lambda D+\frac{1}{2}(F+2 R)\right)=R \cup O,
$$

which is impossible by Theorem 2.7 since $-K_{X} \sim F+2 R \sim_{\mathbb{Q}} D$ and $\lambda<1 / 4$.

Lemma 8.17. Suppose that $I(X)=2.31$. Then $\operatorname{lct}(X)=1 / 3$.

Proof. There is a birational morphism $\alpha: X \rightarrow Q$ such that $Q$ is a smooth quadric hypersurface, and $\alpha$ is a blow up of the quadric $Q$ along a line $L \subset Q$.

Let $H$ be a sufficiently general hyperplane section of the quadric $Q \subset \mathbb{P}^{4}$ that passes through the line $L$, and let $\bar{H}$ be a proper transform of the surface $H$ on the threefold $X$. Then

$$
-K_{X} \sim 3 \bar{H}+2 E,
$$

where $E$ is the exceptional divisor of $\alpha$. In particular, $\operatorname{lct}(X) \leqslant 1 / 3$.

We suppose that $\operatorname{lct}(X)<1 / 3$. Then there exists an effective $\mathbb{Q}$-divisor $D \sim_{\mathbb{Q}}-K_{X}$ such that the log pair $(X, \lambda D)$ is not log canonical for some rational $\lambda<1 / 3$. Then

$$
\varnothing \neq \operatorname{LCS}(X, \lambda D) \subseteq E
$$

since $\operatorname{lct}(Q)=1 / 3$ and $\alpha(D) \sim_{\mathbb{Q}}-K_{Q}$.

The linear system $|\bar{H}|$ defines a $\mathbb{P}^{1}$-bundle $\phi: X \rightarrow \mathbb{P}^{2}$ such that the induced morphism $E \cong \mathbb{F}_{1} \rightarrow \mathbb{P}^{2}$ contracts an irreducible curve $Z \subset E$. One has

$$
\operatorname{LCS}(X, \lambda D)=Z \subset E
$$

by Theorem 2.28, Put

$$
D=\mu E+\Omega,
$$

where $\Omega$ is an effective $\mathbb{Q}$-divisor on $X$ such that $E \not \subset \operatorname{Supp}(\Omega)$. Then

$$
2=D \cdot F=\mu E \cdot F+\Omega \cdot F=\mu+\Omega \cdot F \geqslant \mu,
$$

where $F$ is a general fiber of $\phi$. Note that the log pair

$$
(X, E+\lambda \Omega)
$$

is not $\log$ canonical, because $\lambda<1 / 3$. Then $\left(E,\left.\lambda \Omega\right|_{E}\right)$ is not $\log$ canonical by Theorem 2.20 .

Let $C$ be a fiber of the natural projection $E \rightarrow L$. Then

$$
\left.\Omega\right|_{E} \sim_{\mathbb{Q}} 3 C+(1+\mu) Z,
$$

which implies that $\left(E,\left.\lambda \Omega\right|_{E}\right)$ is $\log$ canonical by Lemma 5.8 , which is a contradiction. 


\section{FAno threefolds With $\rho=3$}

We use the assumptions and notation introduced in section 1,

Lemma 9.1. Suppose that $I(X)=3.1$ and $X$ is general. Then $\operatorname{lct}(X)=3 / 4$.

Proof. There is a double cover

$$
\omega: X \longrightarrow \mathbb{P}^{1} \times \mathbb{P}^{1} \times \mathbb{P}^{1}
$$

branched over a divisor of tridegree $(2,2,2)$. The projection

$$
\mathbb{P}^{1} \times \mathbb{P}^{1} \times \mathbb{P}^{1} \longrightarrow \mathbb{P}^{1}
$$

onto the $i$-th factor induces a morphism $\pi_{i}: X \rightarrow \mathbb{P}^{1}$, whose fibers are del Pezzo surfaces of degree 4 .

Let $R_{1}$ be a singular fiber of the fibration $\pi_{1}$, let $Q$ be a singular point of the surface $R_{1}$, and let $R_{2}$ and $R_{3}$ be fibers of $\pi_{2}$ and $\pi_{3}$ such that

$$
R_{2} \ni Q \in R_{3},
$$

respectively. Then $\operatorname{mult}_{Q}\left(R_{1}+R_{2}+R_{3}\right)=4$, which implies that the log pair

$$
\left(X, \frac{3}{4}\left(R_{1}+R_{2}+R_{3}\right)\right)
$$

is not $\log$ terminal at $Q$. But $-K_{X} \sim R_{1}+R_{2}+R_{3}$. Thus, we see that $\operatorname{lct}(X) \leqslant 3 / 4$.

We suppose that the threefold $X$ satisfies the following generality condition: for an arbitrary point $O \in X$, there is $k \in\{1,2,3\}$ such that

- the fiber $F_{k}$ of the fibration $\pi_{k}$ that contains $O$ is smooth at the point $O$,

- the singularities of the surface $F_{k}$ consist of at most one ordinary double point,

- for every smooth curve $\Gamma \subset F_{k}$ such that $-K_{F_{k}} \cdot \Gamma=1$, we have $O \notin \Gamma$,

- for every smooth curves $\Delta_{1} \subset S_{k} \supset \Delta_{2}$ such that

$$
-K_{F_{k}} \cdot \Delta_{1}=-K_{F_{k}} \cdot \Delta_{2}=2
$$

and $\Delta_{1}+\Delta_{2} \sim-K_{F_{k}}$, we have $O \neq \Delta_{1} \cap \Delta_{2}$.

We suppose that $\operatorname{lct}(X)<3 / 4$. Then there exists an effective $\mathbb{Q}$-divisor $D \sim_{\mathbb{Q}}-K_{X}$ such that the log pair $(X, \lambda D)$ is not $\log$ canonical at some point $P \in X$ for some rational number $\lambda<3 / 4$.

Let $S_{i}$ be a fiber of $\pi_{i}$ such that $P \in S_{i}$. Without loss of generality, we may assume that

- the surface $S_{1}$ is smooth at the point $P$,

- the singularities of the surface $S_{1}$ consist of at most one ordinary double point,

- for every smooth curve $L \subset S_{1}$ such that $-K_{S_{1}} \cdot L=1$, we have $P \notin L$,

- for every smooth curves $C_{1} \subset S_{1} \supset C_{2}$ such that

$$
-K_{S_{1}} \cdot C_{1}=-K_{S_{1}} \cdot C_{2}=2
$$

and $C_{1}+C_{2} \sim-K_{S_{1}}$, we have $P \neq C_{1} \cap C_{2}$.

The surface $S_{1}$ is a del Pezzo surface of degree 4 . One has

$$
D=\mu S_{1}+\Omega,
$$

where $\Omega$ is an effective $\mathbb{Q}$-divisor on $X$ such that $S_{1} \not \subset \operatorname{Supp}(\Omega)$.

Let $\phi: X \rightarrow \mathbb{P}^{1} \times \mathbb{P}^{1}$ be a natural conic bundle induced by the linear system

$$
\left|S_{2}+S_{3}\right|
$$

and let $\Gamma$ be a general fiber of the conic bundle $\phi$. Then

$$
2=D \cdot \Gamma=\mu S_{1} \cdot \Gamma+\Omega \cdot \Gamma=2 \mu+\Omega \cdot \Gamma \geqslant 2 \mu,
$$

which implies that $\mu \leqslant 1$. Then $\left(X, S_{1}+\lambda \Omega\right)$ is not canonical at the point $P$. Hence

$$
\left(S_{1},\left.\lambda \Omega\right|_{S_{1}}\right)
$$

is not $\log$ canonical at the point $P$ by Theorem 2.20. But

$$
\left.\left.\Omega\right|_{S_{1}} \sim{ }_{\mathbb{Q}} D\right|_{S_{1}} \sim \mathbb{Q}-K_{S_{k}},
$$


which is impossible (see Example 5.4 and mind the generality assumption).

Lemma 9.2. Suppose that $I(X)=3.2$ and $X$ is general. Then $\operatorname{lct}(X)=1 / 2$.

Proof. The threefold $X$ is a primitive Fano threefold (see [128, Definition 1.3]). Put

$$
U=\mathbb{P}\left(\mathcal{O}_{\mathbb{P}^{1} \times \mathbb{P}^{1}} \oplus \mathcal{O}_{\mathbb{P}^{1} \times \mathbb{P}^{1}}(-1,-1) \oplus \mathcal{O}_{\mathbb{P}^{1} \times \mathbb{P}^{1}}(-1,-1)\right),
$$

let $\pi: U \rightarrow \mathbb{P}^{1} \times \mathbb{P}^{1}$ be a natural projection, and let $L$ be a tautological line bundle on $U$. Then

$$
X \in\left|2 L+\pi^{*}\left(\mathcal{O}_{\mathbb{P}^{1} \times \mathbb{P}^{1}}(2,3)\right)\right| \text {. }
$$

Let us show that $\operatorname{lct}(X) \leqslant 1 / 2$. Let $E_{1}$ and $E_{2}$ be surfaces in $X$ such that

$$
\pi\left(E_{1}\right) \subset \mathbb{P}^{1} \times \mathbb{P}^{1} \supset \pi\left(E_{2}\right)
$$

are divisors on $\mathbb{P}^{1} \times \mathbb{P}^{1}$ of bi-degree $(1,0)$ and $(0,1)$, respectively. Then

$$
-\left.K_{X} \sim L\right|_{X}+2 E_{1}+E_{2},
$$

which implies that $\operatorname{lct}(X) \leqslant 1 / 2$.

We suppose that $\operatorname{lct}(X)<1 / 2$. Then there exists an effective $\mathbb{Q}$-divisor $D \sim_{\mathbb{Q}}-K_{X}$ such that the log pair $(X, \lambda D)$ is not $\log$ canonical at some point $P \in X$ for some rational number $\lambda<1 / 2$.

It follows from [86, Proposition 3.8] that there is a commutative diagram

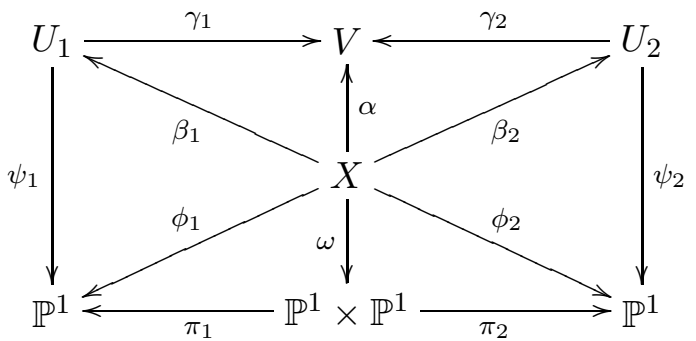

where $V$ is a Fano threefold that has one ordinary double point $O \in V$ such that

$$
\operatorname{Pic}(V)=\mathbb{Z}\left[-K_{V}\right]
$$

and $-K_{V}^{3}=16$, the morphism $\alpha$ contracts a unique surface

$$
\mathbb{P}^{1} \times \mathbb{P}^{1} \cong S \subset X
$$

such that $\left.S \sim L\right|_{X}$ to the point $O \in V$, the morphism $\beta_{i}$ contracts $S$ to a smooth rational curve, the morphism $\gamma_{i}$ contracts the curve $\beta_{i}(S)$ to the point $O \in V$ so that the rational map

$$
\gamma_{2} \circ \gamma_{1}^{-1}: U_{1} \rightarrow U_{2}
$$

is a flop in $\beta_{1}(S) \cong \mathbb{P}^{1}$, the morphism $\psi_{2}$ is a quadric fibration, and the morphisms $\psi_{1}, \phi_{1}, \phi_{2}$ are fibrations whose fibers are del Pezzo surfaces of degree 4, 3 and 6, respectively. The morphisms $\pi_{1}$ and $\pi_{2}$ are natural projections, and $\omega=\left.\pi\right|_{X}$. Then

$$
\mathrm{Cl}(V)=\mathbb{Z}\left[\alpha\left(E_{1}\right)\right] \oplus \mathbb{Z}\left[\alpha\left(E_{2}\right)\right],
$$

and $\omega$ is a conic bundle. The curve $\beta_{1}(S)$ is a section of $\psi_{1}$, and $\beta_{2}(S)$ is a 2-section of $\psi_{2}$.

We assume that the threefold $X$ satisfies the following mild generality condition: every singular fiber of the del Pezzo fibration $\phi_{2}$ has at most $\mathbb{A}_{1}$ singularities.

Applying Lemma 2.26 to the fibration $\phi_{1}$, we see that

$$
\varnothing \neq \operatorname{LCS}(X, \lambda D) \subseteq S_{1}
$$

where $S_{1}$ is a singular fiber of the del Pezzo fibration $\phi_{1}$, because the global log canonical threshold of a smooth del Pezzo surface of degree 6 is equal to 1/2 by Example 1.18 .

Applying Lemma 2.26 to $\phi_{2}$, we obtain a contradiction by Example 1.38.

Lemma 9.3. Suppose that $I(X)=3.3$ and $X$ is general. Then $\operatorname{lct}(X)=2 / 3$. 
Proof. The threefold $X$ is a divisor on $\mathbb{P}^{1} \times \mathbb{P}^{1} \times \mathbb{P}^{2}$ of tridegree $(1,1,2)$. In particular,

$$
-K_{X} \sim \pi_{1}^{*}\left(\mathcal{O}_{\mathbb{P}^{1}}(1)\right)+\pi_{2}^{*}\left(\mathcal{O}_{\mathbb{P}^{1}}(1)\right)+\phi^{*}\left(\mathcal{O}_{\mathbb{P}^{2}}(1)\right),
$$

where $\pi_{1}: X \rightarrow \mathbb{P}^{1}$ and $\pi_{1}: X \rightarrow \mathbb{P}^{1}$ are fibrations into del Pezzo surfaces of degree 4 that are induced by the projections of the variety $\mathbb{P}^{1} \times \mathbb{P}^{1} \times \mathbb{P}^{2}$ onto its first and second factor, respectively, and $\phi: X \rightarrow \mathbb{P}^{2}$ is conic bundle that is induced by the projection $\mathbb{P}^{1} \times \mathbb{P}^{1} \times \mathbb{P}^{2} \rightarrow \mathbb{P}^{2}$.

Let $\alpha_{2}: X \rightarrow \mathbb{P}^{1} \times \mathbb{P}^{2}$ be a birational morphism induced by the linear system

$$
\left|\pi_{2}^{*}\left(\mathcal{O}_{\mathbb{P}^{1}}(1)\right)+\phi^{*}\left(\mathcal{O}_{\mathbb{P}^{2}}(1)\right)\right|,
$$

let $H_{i} \in\left|\pi_{i}^{*}\left(\mathcal{O}_{\mathbb{P}^{1}}(1)\right)\right|$ and $R \in\left|\phi^{*}\left(\mathcal{O}_{\mathbb{P}^{2}}(1)\right)\right|$ be general surfaces. Then

$$
H_{1} \sim H_{2}+2 R-E_{2}
$$

where $E_{2}$ is the exceptional divisor of the birational morphism $\alpha_{2}$. Hence

$$
-K_{X} \sim H_{1}+H_{2}+R \sim \mathbb{Q} \frac{3}{2} H_{1}+\frac{1}{2} H_{2}+\frac{1}{2} E_{2},
$$

which implies that $\operatorname{lct}(X) \leqslant 2 / 3$.

We suppose that the threefold $X$ satisfies the following generality conditions: for an arbitrary point $O \in X$, there is $k \in\{1,2\}$ such that

- the fiber $F_{k}$ of the fibration $\pi_{k}$ that contains the point $O$ is smooth at the point $O$,

- the singularities of the surface $F_{k}$ consist of at most one ordinary double point,

- for every smooth curve $\Gamma \subset F_{k}$ such that $-K_{F_{k}} \cdot \Gamma=1$, we have $O \notin \Gamma$ if $\operatorname{Sing}\left(F_{k}\right) \neq \varnothing$.

We suppose that $\operatorname{lct}(X)<2 / 3$. Then there exists an effective $\mathbb{Q}$-divisor $D \sim_{\mathbb{Q}}-K_{X}$ such that the log pair $(X, \lambda D)$ is not $\log$ canonical at some point $P \in X$ for some rational number $\lambda<2 / 3$.

Let $S_{i}$ be a fiber of $\pi_{i}$ such that $P \in S_{i}$. Then we may assume that

- the surface $S_{1}$ is smooth at the point $P$,

- the singularities of the surface $S_{1}$ consist of at most one ordinary double point,

- for every smooth curve $L \subset S_{1}$ such that $-K_{S_{1}} \cdot L=1$, we have $P \notin L$ if $\operatorname{Sing}\left(S_{1}\right) \neq \varnothing$.

Put $D=\mu S_{1}+\Omega$, where $\Omega$ is an effective $\mathbb{Q}$-divisor such that $S_{1} \not \subset \operatorname{Supp}(\Omega)$. Then

$$
\left(H_{2},\left.\lambda \mu S_{1}\right|_{H_{2}}+\left.\lambda \Omega\right|_{H_{2}}\right)
$$

is $\log$ canonical because $\operatorname{lct}\left(H_{2}\right)=2 / 3$. Thus, we see that $\mu \leqslant 1 / \lambda$. Hence

$$
\left(S_{1},\left.\lambda \Omega\right|_{S_{1}}\right)
$$

is not $\log$ canonical at the point $P$ by Theorem 2.20 , But

$$
\left.\Omega\right|_{S_{1}} \sim \mathbb{Q}-K_{S_{1}}
$$

which is impossible (see Example 5.4).

Lemma 9.4. Suppose that $I(X)=3.4$. Then $\operatorname{lct}(X)=1 / 2$.

Proof. Let $O$ be a point in $\mathbb{P}^{2}$. Then there is a commutative diagram

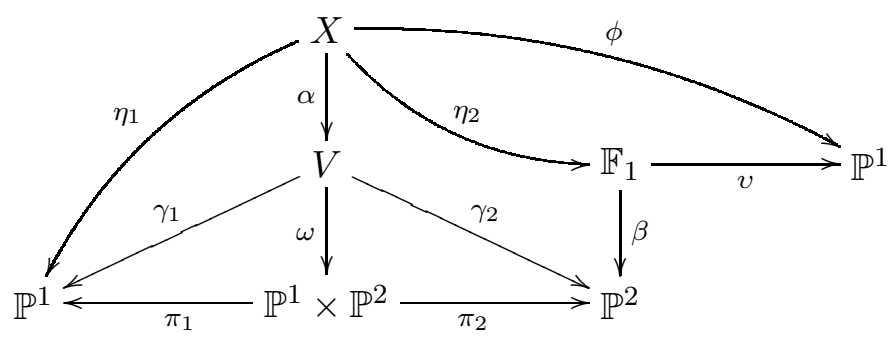

such that $\pi_{i}$ and $v$ are natural projections, $\omega$ is a double cover branched over a divisor $B \subset \mathbb{P}^{1} \times \mathbb{P}^{2}$ of bi-degree $(2,2)$, the morphism $\gamma_{1}$ is a fibration into quadrics, $\gamma_{2}$ and $\eta_{2}$ are conic bundles, the morphism $\beta$ is a blow up of the point $O$, the morphism $\alpha$ is a blow up of a smooth curve that 
is a fiber of $\gamma_{2}$ over the point $O$, the morphism $\eta_{1}$ is a fibration into del Pezzo surfaces of degree 6 , and $\phi$ is a fibration into del Pezzo surfaces of degree 4.

Let $H$ be a general fiber of $\eta_{1}$, and let $S$ be a general fiber of $\phi$. Then

$$
-K_{X} \sim H+2 S+E,
$$

where $E$ is the exceptional divisor of $\alpha$. Thus, we see that $\operatorname{lct}(X) \leqslant 1 / 2$.

We suppose that $\operatorname{lct}(X)<1 / 2$. Then there exists an effective $\mathbb{Q}$-divisor $D \sim_{\mathbb{Q}}-K_{X}$ such that the log pair $(X, \lambda D)$ is not log canonical for some positive rational number $\lambda<1 / 2$. Then

$$
\varnothing \neq \operatorname{LCS}(X, \lambda D) \subseteq E,
$$

because $\alpha(D) \sim_{\mathbb{Q}}-K_{V}$ and $\operatorname{lct}(V)=1 / 2$ by Lemma 8.7

Let $\Gamma$ be a fiber of $\eta_{2}$ such that $\Gamma \cap \operatorname{LCS}(X, \lambda D) \neq \varnothing$. Then

$$
\Gamma \subseteq \operatorname{LCS}(X, \lambda D) \subseteq E,
$$

by Theorem 2.28. Hence $\left(H,\left.\lambda D\right|_{H}\right)$ is not log canonical at the points $H \cap \Gamma$. But

$$
\left.D\right|_{H} \sim_{\mathbb{Q}}-\left.K_{X}\right|_{H} \sim-K_{H}
$$

and $\operatorname{lct}(H)=1 / 2$, because $H$ is a del Pezzo surface of degree 6 , which is a contradiction.

Lemma 9.5. Suppose that $I(X)=3.5$ and $X$ is general. Then $\operatorname{lct}(X)=1 / 2$.

Proof. There is a birational morphism $\alpha: X \rightarrow \mathbb{P}^{1} \times \mathbb{P}^{2}$ that contracts a surface $E \subset X$ to a curve $C \subset \mathbb{P}^{1} \times \mathbb{P}^{2}$ of bidegree $(5,2)$. Let $\pi_{1}: \mathbb{P}^{1} \times \mathbb{P}^{2} \rightarrow \mathbb{P}^{1}$ and $\pi_{2}: \mathbb{P}^{1} \times \mathbb{P}^{2} \rightarrow \mathbb{P}^{2}$ be natural projections. There is

$$
Q \in\left|\pi_{2}^{*}\left(\mathcal{O}_{\mathbb{P}^{1}}(2)\right)\right|
$$

such that $C \subset Q$. Let $H_{1}$ be a general fiber of $\pi_{1}$, let $H_{2}$ be a surface in $\left|\pi_{2}^{*}\left(\mathcal{O}_{\mathbb{P}^{1}}(1)\right)\right|$. We have

$$
-K_{X} \sim 2 \bar{H}_{1}+\bar{H}_{2}+\bar{Q},
$$

where $\bar{H}_{1}, \bar{H}_{2}, \bar{Q} \subset X$ are proper transforms of $H_{1}, H_{2}, Q$, respectively. In particular, lct $(X) \leqslant$ $1 / 2$.

We suppose that $X$ satisfies the following generality condition: every fiber $F$ of $\pi_{1} \circ \alpha$ is singular at most at one ordinary double point.

Assume that $\operatorname{lct}(X)<1 / 2$. Then there exists an effective $\mathbb{Q}$-divisor $D \sim_{\mathbb{Q}}-K_{X}$ such that the log pair $(X, \lambda D)$ is not log canonical for some positive rational number $\lambda<1 / 2$.

Let $S$ be an irreducible surface on the threefold $X$. Put

$$
D=\mu S+\Omega,
$$

where $\Omega$ is an effective $\mathbb{Q}$-divisor such that $S \not \subset \operatorname{Supp}(\Omega)$. Then

$$
\left(\bar{H}_{1},\left.\frac{1}{2}(\mu S+\Omega)\right|_{\bar{H}_{1}}\right)
$$

is $\log$ canonical (see Example 1.18). Thus, either $\mu \leqslant 2$, or $S$ is a fiber of $\pi_{1} \circ \alpha$.

Let $\Gamma \cong \mathbb{P}^{1}$ be a general fiber of the conic bundle $\pi_{2} \circ \alpha$. Then

$$
2=D \cdot \Gamma=\mu S \cdot \Gamma+\Omega \cdot \Gamma \geqslant \mu S \cdot \Gamma,
$$

which implies that $\mu \leqslant 2$ in the case when $S$ is a fiber of $\pi_{1} \circ \alpha$.

We see that the set $\mathbb{L} \mathbb{C} S(X, \lambda D)$ contains no surfaces. Now, applying Lemma 2.26 to $\pi_{1} \circ \alpha$, we obtain a contradiction with Example 5.4.

Lemma 9.6. Suppose that $I(X)=3.6$ and $X$ is general. Then $\operatorname{lct}(X)=1 / 2$.

Proof. Let $\varepsilon: V \rightarrow \mathbb{P}^{3}$ be a blow up of a line $L \subset \mathbb{P}^{3}$. Then

$$
V \cong \mathbb{P}\left(\mathcal{O}_{\mathbb{P}^{1}} \oplus \mathcal{O}_{\mathbb{P}^{1}} \oplus \mathcal{O}_{\mathbb{P}^{1}}(1)\right)
$$


and there is a natural $\mathbb{P}^{2}$-bundle $\eta: V \rightarrow \mathbb{P}^{1}$. There is a smooth elliptic curve $C \subset \mathbb{P}^{3}$ of degree 4 such that $L \cap C=\varnothing$ and there is a commutative diagram

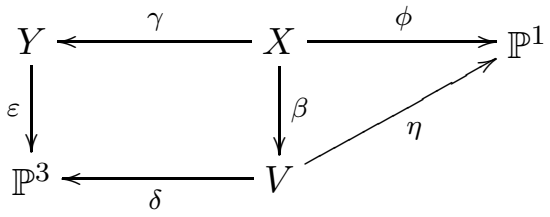

where $\delta$ is a blow up of $C$, the morphism $\beta$ is a blow up of the proper transform of the line $L$, the morphism $\gamma$ is a blow up of the proper transform of the curve $C$, and $\phi$ is a del Pezzo fibration.

We suppose that $X$ satisfies the following generality condition: for every fiber $F$ of $\phi$, the surface $F$ has at most one singular point that is an ordinary double point of the surface $F$.

Let $E$ and $G$ be the exceptional surfaces of $\beta$ and $\gamma$, respectively, let $H \subset \mathbb{P}^{3}$ be a general plane that passes through $L$, and let $Q \subset \mathbb{P}^{3}$ a quadric surface that passes through $C$. Then

$$
-K_{X} \sim 2 \bar{H}+\bar{Q}+E,
$$

where $\bar{H} \subset X \supset \bar{Q}$ are proper transforms of $H$ and $Q$, respectively. We have $\operatorname{lct}(X) \leqslant 1 / 2$.

We suppose that $\operatorname{lct}(X)<1 / 2$. Then there exists an effective $\mathbb{Q}$-divisor $D \sim_{\mathbb{Q}}-K_{X}$ such that the log pair $(X, \lambda D)$ is not log canonical for some positive rational number $\lambda<1 / 2$.

It follows from Lemma 8.11 that $\operatorname{lct}(V)=1 / 2$. Therefore, we see that

$$
\varnothing \neq \operatorname{LCS}(X, \lambda D) \subseteq G .
$$

Note that every fiber of the fibration $\phi$ is a del Pezzo surface of degree 5 that has at most one ordinary double point. Thus, applying Lemma 2.26 to $\phi$, we obtain a contradiction with Example 5.3.

Lemma 9.7. Suppose that $I(X)=3.7$ and $X$ is general. Then $\operatorname{lct}(X)=1 / 2$.

Proof. Let $W$ be a divisor on $\mathbb{P}^{2} \times \mathbb{P}^{2}$ of bi-degree $(1,1)$. Then $-K_{W} \sim 2 H$, where $H$ is a Cartier divisor on $W$. There is a commutative diagram

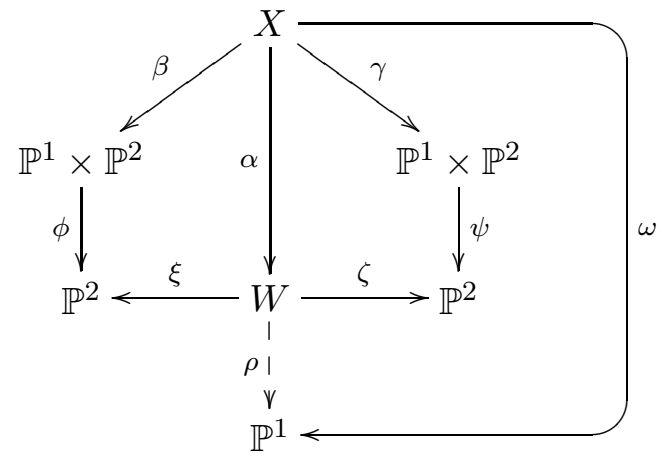

where $\phi$ and $\psi$ are natural projections, $\alpha$ is a blow up of a smooth curve $C \subset W$ such that

$$
C=H_{1} \cap H_{2},
$$

where $H_{1} \neq H_{2}$ are surfaces in $|H|$, the map $\rho$ is induced by the pencil generated by $H_{1}$ and $H_{2}$, the morphism $\omega$ is a del Pezzo fibration of degree 6 , the morphisms $\zeta$ and $\xi$ are $\mathbb{P}^{1}$-bundles, while $\beta$ and $\gamma$ contract surfaces $\bar{M}_{1} \subset X \supset \bar{M}_{2}$ such that $\phi \circ \beta\left(\bar{M}_{1}\right)=\xi(C)$ and $\psi \circ \gamma\left(\bar{M}_{2}\right)=\zeta(C)$.

Note that $\operatorname{lct}(X) \leqslant 1 / 2$, because

$$
-K_{X} \sim 2 \bar{H}_{1}+E
$$

where $\bar{H}_{1} \subset X$ is the proper transform of $H_{1}$, and $E$ is the exceptional surface of $\alpha$.

We suppose that $X$ satisfies the following generality condition: all singular fibers of the fibration $\omega$ satisfy the hypotheses of Lemma 5.5 .

Assume that $\operatorname{lct}(X)<1 / 2$. Then there exists an effective $\mathbb{Q}$-divisor $D \sim_{\mathbb{Q}}-K_{X}$ such that the log pair $(X, \lambda D)$ is not log canonical for some positive rational number $\lambda<1 / 2$. Then

$$
\varnothing \neq \operatorname{LCS}(X, \lambda D) \subseteq E,
$$


because $\operatorname{lct}(W)=1 / 2$ by Theorem 7.1 . Applying Lemma 2.26, we see that

$$
\varnothing \neq \operatorname{LCS}(X, \lambda D) \subseteq E \cap F,
$$

where $F$ is a singular fiber of $\omega$. Note that $F$ is a del Pezzo surface of degree 6 . Put

$$
D=\mu F+\Omega,
$$

where $\Omega$ is an effective $\mathbb{Q}$-divisor such that $F \not \subset \operatorname{Supp}(\Omega)$. Then

$$
\left.\left.\Omega\right|_{F} \sim_{\mathbb{Q}} D\right|_{F} \sim_{\mathbb{Q}}-K_{F}
$$

and the surface $F$ is smooth along the curve $E \cap F$. But the log pair $\left(F,\left.\lambda \Omega\right|_{F}\right)$ is not log canonical at some point $P \in E \cap F$ by Theorem 2.20, which is impossible by Lemma 5.5.

Remark 9.8. Let us use the notation and assumptions of the proof of Lemma 9.7. Then

$$
\varnothing \neq \operatorname{LCS}(X, \lambda D) \subseteq E \cap F,
$$

where $F$ is a singular fiber of the fibration $\omega$. Applying Theorem 2.28 to $\phi$ and $\psi$, we see that

$$
\varnothing \neq \operatorname{LCS}(X, \lambda D) \subseteq E \cap F \cap \bar{M}_{1} \cap \bar{M}_{2},
$$

by Lemma 2.29. Regardless to how singular $F$ is, if the threefold $X$ is sufficiently general, then

$$
E \cap F \cap \bar{M}_{1} \cap \bar{M}_{2}=\varnothing,
$$

which implies that an alternative generality condition can be used in Lemma 9.7 .

Lemma 9.9. Suppose that $I(X)=3.8$ and $X$ is general. Then $\operatorname{lct}(X)=1 / 2$.

Proof. Let $\pi_{1}: \mathbb{F}_{1} \times \mathbb{P}^{2} \rightarrow \mathbb{F}_{1}$ and $\pi_{2}: \mathbb{F}_{1} \times \mathbb{P}^{2} \rightarrow \mathbb{P}^{2}$ be natural projections. Then

$$
X \in\left|\left(\alpha \circ \pi_{1}\right)^{*}\left(\mathcal{O}_{\mathbb{P}^{2}}(1)\right) \otimes \pi_{2}^{*}\left(\mathcal{O}_{\mathbb{P}^{2}}(2)\right)\right|,
$$

where $\alpha: \mathbb{F}_{1} \rightarrow \mathbb{P}^{2}$ is a blow up of a point. Let $H$ be a surface in $\left|\pi_{2}^{*}\left(\mathcal{O}_{\mathbb{P}^{2}}(1)\right)\right|$. Then

$$
-K_{X} \sim E+2 L+H
$$

where $E \subset X \supset L$ are irreducible surfaces such that $\pi_{1}(E) \subset \mathbb{F}_{1}$ is the exceptional curve of $\alpha$, and $\pi_{1}(L) \subset \mathbb{F}_{1}$ is a fiber of the natural projection $\mathbb{F}_{1} \rightarrow \mathbb{P}^{2}$. We have $\operatorname{lct}(X) \leqslant 1 / 2$.

The projection $\pi_{1}$ induces a fibration $\phi: X \rightarrow \mathbb{P}^{1}$ into del Pezzo surfaces of degree 5 .

We suppose that $X$ satisfies the following generality condition: for every fiber $F$ of $\phi$, the surface $F$ has at most one singular point that is an ordinary double point of the surface $F$.

Assume that $\operatorname{lct}(X)<1 / 2$. Then there exists an effective $\mathbb{Q}$-divisor $D \sim_{\mathbb{Q}}-K_{X}$ such that the log pair $(X, \lambda D)$ is not log canonical for some positive rational number $\lambda<1 / 2$.

Applying Lemma 2.26 to the morphism $\phi$, we obtain a contradiction with Example 5.3

Lemma 9.10. Suppose that $I(X)=3.9$. Then $\operatorname{lct}(X)=1 / 3$.

Proof. Let $O_{1} \in V_{1} \cong V_{2} \ni O_{2}$ be singular points of $V_{1} \cong V_{2} \cong \mathbb{P}(1,1,1,2)$, respectively, let

$$
O_{1} \notin S_{1} \in\left|\mathcal{O}_{\mathbb{P}(1,1,1,2)}(2)\right|
$$

be a smooth surface, and let $C_{1} \subset S_{1} \cong \mathbb{P}^{2}$ be a smooth quartic curve. Then the diagram

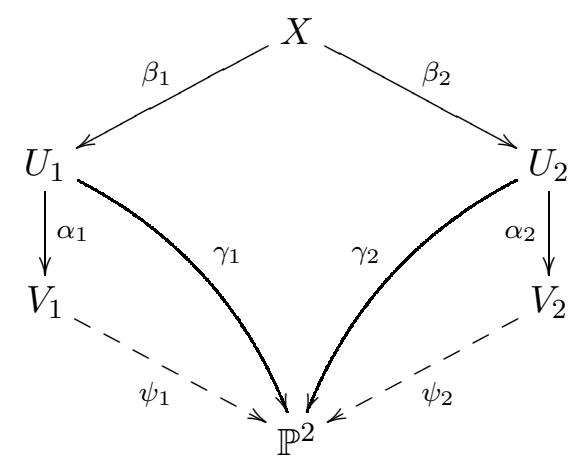


commutes, where $\psi_{i}$ is a natural projection, $\alpha_{i}$ is a blow up of the point $O_{i}$ with weights $(1,1,1)$, the morphism $\gamma_{i}$ is a $\mathbb{P}^{1}$-bundle, and $\beta_{i}$ is a birational morphism that contracts a surface

$$
\mathbb{P}^{1} \times C_{1} \cong G_{i} \subset X
$$

to a smooth curve $C_{1} \cong C_{i} \subset U_{i}$.

Let $E_{i} \subset X$ be the proper transform of the exceptional divisor of $\alpha_{i}$. Then

$$
S_{1}=\alpha_{1} \circ \beta_{1}\left(E_{2}\right) \subset V_{1} \cong \mathbb{P}(1,1,1,2) \cong V_{2} \supset \alpha_{2} \circ \beta_{2}\left(E_{1}\right)
$$

are surfaces in $\left|\mathcal{O}_{\mathbb{P}(1,1,1,2)}(2)\right|$ that contain the curves $C_{1}$ and $C_{2}$, respectively. On the other hand,

$$
\alpha_{1} \circ \beta_{1}\left(G_{2}\right) \subset V_{1} \cong \mathbb{P}(1,1,1,2) \cong V_{2} \supset \alpha_{2} \circ \beta_{2}\left(G_{1}\right)
$$

are surfaces in $\left|\mathcal{O}_{\mathbb{P}(1,1,1,2)}(4)\right|$ that contain $O_{1} \cup C_{1}$ and $O_{2} \cup C_{2}$, respectively.

Let $\bar{H} \subset X$ be the proper transform of a general surface in $\left|\mathcal{O}_{\mathbb{P}(1,1,1,2)}(1)\right|$. Then

$$
-K_{X} \sim 3 \bar{H}+E_{2}+E_{1}
$$

which gives $\operatorname{lct}(X) \leqslant 1 / 3$.

Suppose that $\operatorname{lct}(X)<1 / 3$. Then there is an effective $\mathbb{Q}$-divisor

$$
D \sim_{\mathbb{Q}}-K_{X} \sim_{\mathbb{Q}} \frac{5}{2}\left(G_{1}+G_{2}\right)-5\left(E_{1}+E_{2}\right)
$$

such that the log pair $(X, \lambda D)$ is not $\log$ canonical for some $\lambda<1 / 3$. Put

$$
D=\mu_{1} E_{1}+\mu_{2} E_{2}+\Omega
$$

where $\Omega$ is an effective $\mathbb{Q}$-divisor on $X$ such that

$$
E_{1} \not \subset \operatorname{Supp}(\Omega) \nsupseteq E_{2} \text {. }
$$

Let $\Gamma$ be a general fiber of the conic bundle $\gamma_{1} \circ \beta_{1}$. Then

$$
2=\Gamma \cdot D=\Gamma \cdot\left(\mu_{1} E_{1}+\mu_{2} E_{2}+\Omega\right)=\mu_{1}+\mu_{2}+\Gamma \cdot \Omega \geqslant \mu_{1}+\mu_{2},
$$

and without loss of generality we may assume that $\mu_{1} \leqslant \mu_{2}$. Then $\mu_{1} \leqslant 1$.

Suppose that there is a surface $S \in \mathbb{L} \mathbb{C} S(X, \lambda D)$. Then $S \neq E_{1}$ and $S \neq G_{1}$, because $\alpha_{2} \circ \beta_{2}\left(G_{1}\right) \in\left|\mathcal{O}_{\mathbb{P}(1,1,1,2)}(4)\right|$ and $\alpha_{2} \circ \beta_{2}(D) \in\left|\mathcal{O}_{\mathbb{P}(1,1,1,2)}(5)\right|$. Hence $S \cap E_{1} \neq \varnothing$. But

$$
-\left.\frac{1}{3} K_{E_{1}} \sim_{\mathbb{Q}} D\right|_{E_{1}}=-\frac{2 \mu_{1}}{3} K_{E_{1}}+\left.\Omega\right|_{E_{1}}
$$

and $E_{1} \cong \mathbb{P}^{2}$, which is impossible by Theorem 2.20, because $\lambda<1 / 3=\operatorname{lct}\left(\mathbb{P}^{2}\right)$.

We see that the set $\mathbb{L} \mathbb{C S}(X, \lambda D)$ contains no surfaces. Let $P \in \operatorname{LCS}(X, \lambda D)$ be a point. Suppose that $P \notin G_{1}$. Let $Z$ be a fiber of $\gamma_{1}$ such that $\beta_{1}(P) \in Z$. Then

$$
Z \subseteq \operatorname{LCS}\left(U_{1}, \lambda \beta_{1}(D)\right)
$$

by Theorem 2.28, Put $\bar{E}_{1}=\beta_{1}\left(E_{1}\right)$. Then we have

$$
Z \cap \bar{E}_{1} \in \operatorname{LCS}\left(\bar{E}_{1},\left.\lambda \Omega\right|_{\bar{E}_{1}}\right)
$$

by Theorem 2.20, which is impossible by Lemma 2.8 , because $\mu_{1} \leqslant 1$. Hence $\operatorname{LCS}(X, \lambda D) \subsetneq G_{1}$.

Suppose that $\operatorname{LCS}(X, \lambda D) \subseteq G_{1} \cap G_{2}$. Then

$$
|\operatorname{LCS}(X, \lambda D)|=1
$$

by Lemma 2.14 and Theorem 2.7. One has

$$
\operatorname{LCS}(X, \lambda D) \cup \bar{H} \subseteq \operatorname{LCS}\left(X, \lambda D+\frac{1}{3}\left(E_{2}+E_{2}\right)+\bar{H}\right) \subset \operatorname{LCS}(X, \lambda D) \cup \bar{H} \cup E_{1} \cup E_{1},
$$

which contradicts Theorem 2.7, because $\bar{H}$ is a general surface in $\left|\left(\beta_{1} \circ \gamma_{1}\right)^{*}\left(\mathcal{O}_{\mathbb{P}^{2}}(1)\right)\right|$ and

$$
\lambda D+\frac{1}{3}\left(E_{2}+E_{2}\right)+\bar{H} \sim_{\mathbb{Q}}(\lambda-1 / 3) K_{X} .
$$


Thus, we see that $G_{1} \supsetneq \mathbb{L} \mathbb{C S}(X, \lambda D) \nsubseteq G_{1} \cap G_{2}$. Then

$$
\varnothing \neq \operatorname{LCS}\left(U_{2}, \lambda \beta_{2}(D)\right) \subsetneq \beta_{2}\left(G_{1}\right),
$$

and it follows from Theorems 2.7 and 2.28 that there is a fibre $L$ of the fibration $\gamma_{2}$ such that

$$
\operatorname{LCS}\left(U_{2}, \lambda \beta_{2}(D)\right)=L \text {. }
$$

Let $B$ be a general surface in $\left|\alpha_{2}^{*}\left(\mathcal{O}_{\mathbb{P}(1,1,1,2)}(2)\right)\right|$. Then $\left.\beta_{2}(D)\right|_{B} \sim_{\mathbb{Q}} \mathcal{O}_{\mathbb{P}^{2}}(5)$ and $B \cong \mathbb{P}^{2}$. But

$$
\operatorname{LCS}\left(B,\left.\lambda \beta_{2}(D)\right|_{B}\right)=L \cap B
$$

and $|L \cap B|=1$, which is impossible by Lemma 2.8, because $\lambda<1 / 3$.

Lemma 9.11. Suppose that $I(X)=3.10$. Then $\operatorname{lct}(X)=1 / 2$.

Proof. Let $Q \subset \mathbb{P}^{4}$ be a smooth quadric hypersurface. Let $C_{1} \subset Q \supset C_{2}$ be disjoint (irreducible) conics. Then there is a commutative diagram

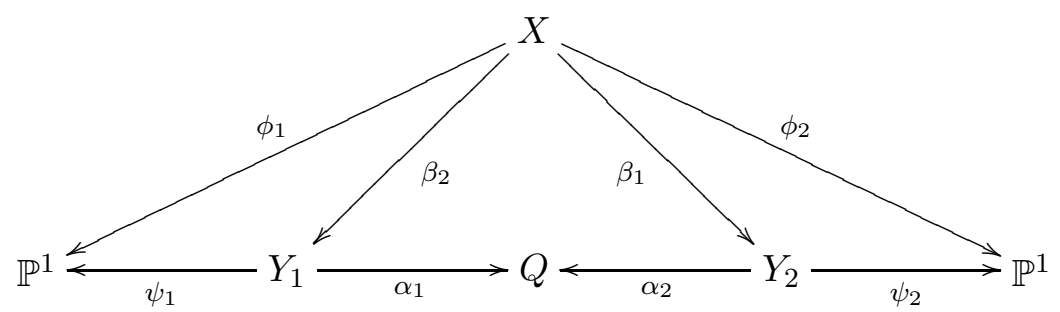

where the morphism $\alpha_{i}$ is a blow up along the conic $C_{i}$, the morphism $\beta_{i}$ is a blow up along the proper transform of the conic $C_{i}$, the morphism $\psi_{i}$ is a natural fibration into quadric surfaces, and $\phi_{i}$ is fibration, whose general fiber is isomorphic to a smooth del Pezzo surfaces of degree 6 .

Let $E_{i}$ be the exceptional divisor of the morphism $\beta_{i}$, and let $H_{i}$ be a sufficiently general hyperplane section of the quadric $Q$ that passes through the conic $C_{i}$. Then

$$
-K_{X} \sim \bar{H}_{1}+2 \bar{H}_{2}+E_{2},
$$

where $\bar{H}_{i} \subset X$ is the proper transform of the surface $H_{i}$. We see that $\operatorname{lct}(X) \leqslant 1 / 2$.

We suppose that $\operatorname{lct}(X)<1 / 2$. Then there exists an effective $\mathbb{Q}$-divisor $D \sim_{\mathbb{Q}}-K_{X}$ such that the log pair $(X, \lambda D)$ is not log canonical for some positive rational number $\lambda<1 / 2$.

Using Example 1.18 and applying Lemma 2.28, we see that

$$
\varnothing \neq \operatorname{LCS}(X, \lambda D) \subseteq S_{1} \cap S_{2},
$$

where $S_{i}$ is a singular fiber of $\phi_{i}$. Hence, the set $\mathbb{L} \mathbb{C S}(X, \lambda D)$ contains no surfaces.

It follows from Theorem 2.7 that either $\operatorname{LCS}(X, \lambda D)$ is a point in $E_{1} \cup E_{2}$, or

$$
\operatorname{LCS}(X, \lambda D) \cap\left(X \backslash\left(E_{1} \cup E_{2}\right)\right) \neq \varnothing
$$

which implies that we may assume that $\operatorname{LCS}(X, \lambda D)$ is a point in $E_{1}$ by Lemma 2.10 .

Since $\beta_{2}$ is an isomorphism on $X \backslash E_{2}$, we see that

$$
P \in \operatorname{LCS}\left(Y_{1}, \lambda \beta_{2}(D)\right) \subset P \cup \beta_{2}\left(E_{2}\right)
$$

for some point $P \in E_{1}$. Then $\operatorname{LCS}\left(Y_{1}, \lambda \beta_{2}(D)\right)=P$ by Theorem 2.7 , because $P \notin \beta_{2}\left(E_{2}\right)$.

Let $H$ be a general hyperplane section of the quadric $Q$. Then

$$
-K_{Y_{1}} \sim \tilde{H}_{1}+2 \tilde{H} \sim_{\mathbb{Q}} \beta_{2}(D),
$$

where $\tilde{H} \subset Y_{1} \supset \tilde{H}_{1}$ are proper transforms of $H$ and $H_{1}$, respectively. But

$$
\operatorname{LCS}\left(Y_{1}, \lambda \beta_{2}(D)+\frac{1}{2}\left(\tilde{H}_{1}+2 \tilde{H}\right)\right)=P \cup \tilde{H},
$$

which is impossible by Theorem 2.7, because $\lambda<1 / 2$.

Lemma 9.12. Suppose that $I(X)=3.11$. Then $\operatorname{lct}(X)=1 / 2$. 
Proof. Let $O \in \mathbb{P}^{3}$ be a point, let $\delta: V_{7} \rightarrow \mathbb{P}^{3}$ be a blow up of the point $O$, and let $E$ be the exceptional divisor of $\delta$. Then

$$
V_{7} \cong \mathbb{P}\left(\mathcal{O}_{\mathbb{P}^{2}} \oplus \mathcal{O}_{\mathbb{P}^{2}}(1)\right)
$$

there is a natural $\mathbb{P}^{1}$-bundle $\eta: V_{7} \rightarrow \mathbb{P}^{2}$, and $E$ is a section of $\eta$. There is a linearly normal elliptic curve $O \in C \mid$ subset $\mathbb{P}^{3}$ such that the diagram

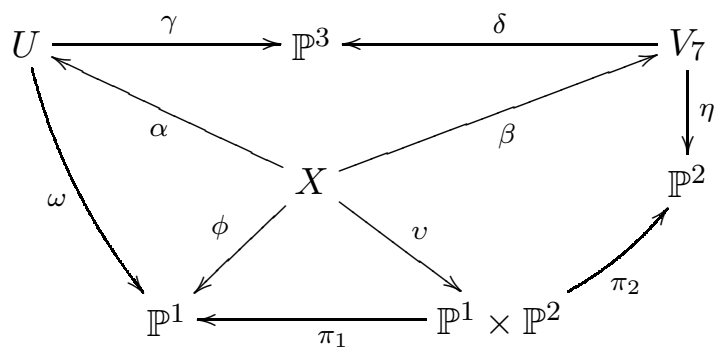

commutes, where $\pi_{1}$ and $\pi_{2}$ are natural projections, the morphism $\gamma$ contracts a surface

$$
C \times \mathbb{P}^{1} \cong G \subset U
$$

to the curve $C$, the morphism $\alpha$ is a blow up of the fiber of the morphism $\gamma$ over the point $O \in \mathbb{P}^{3}$, the morphism $\beta$ is a blow up of the proper transform of $C$, the morphism $\omega$ is a fibration into quadric surfaces, the morphism $\phi$ is a fibration into del Pezzo surfaces of degree 7 , and $v$ contracts a surface

$$
C \times \mathbb{P}^{1} \cong F \subset X
$$

to an elliptic curve $Z \subset \mathbb{P}^{1} \times \mathbb{P}^{2}$ such that $-K_{\mathbb{P}^{1} \times \mathbb{P}^{2}} \cdot Z=13$ and $Z \cong C$.

Let $H_{1}$ be a general fiber of $\phi$, and let $H_{2}$ be a general surface in $\left|(\eta \circ \beta)^{*}\left(\mathcal{O}_{\mathbb{P}^{2}}(1)\right)\right|$. Then

$$
-K_{X} \sim H_{1}+2 H_{2}
$$

which implies that $\operatorname{lct}(X) \leqslant 1 / 2$.

We suppose that $\operatorname{lct}(X)<1 / 2$. Then there exists an effective $\mathbb{Q}$-divisor $D \sim_{\mathbb{Q}}-K_{X}$ such that the $\log$ pair $(X, \lambda D)$ is not log canonical for some positive rational number $\lambda<1 / 2$. Note that

$$
\varnothing \neq \operatorname{LCS}(X, \lambda D) \subseteq \bar{E},
$$

where $\bar{E}$ is the exceptional divisor of $\alpha$, because $\operatorname{lct}(U)=1 / 2$ by Lemma 8.11.

Let $\Gamma \cong \mathbb{P}^{2}$ be the general fiber of $\pi_{2} \circ v$. Then

$$
2=-K_{X} \cdot \Gamma=D \cdot \Gamma=2 \bar{E} \cdot \Gamma,
$$

which implies that $\bar{E} \not \subset \operatorname{LCS}(X, \lambda D)$. Applying Lemma 2.26 to the log pair

$$
\left(V_{7}, \lambda \beta(D)\right)
$$

we see that $\operatorname{LCS}(X, \lambda D) \subseteq \bar{E} \cap G$. Applying Lemma 2.29 to the log pair

$$
\left(\mathbb{P}^{1} \times \mathbb{P}^{2}, \lambda v(D)\right)
$$

we see that $\operatorname{LCS}(X, \lambda D)=\bar{E} \cap F \cap G$, where $|\bar{E} \cap F \cap G|=1$. Hence

$$
\operatorname{LCS}\left(X, \lambda D+H_{2}\right)=\operatorname{LCS}(X, \lambda D) \cup H_{2},
$$

and $H_{2} \cap \operatorname{LCS}(X, \lambda D)=\varnothing$. But the divisor

$$
-\left(K_{X}+\lambda D+H_{2}\right)=\left(\lambda-\frac{1}{2}\right) K_{X}+\frac{1}{2} H_{1}
$$

is ample, which is impossible by Theorem 2.7 .

Lemma 9.13. Suppose that $I(X)=3.12$. Then $\operatorname{lct}(X)=1 / 2$. 
Proof. Let $\varepsilon: V \rightarrow \mathbb{P}^{3}$ be a blow up of a line $L \subset \mathbb{P}^{3}$. There is a natural $\mathbb{P}^{2}$-bundle $\eta: V \rightarrow \mathbb{P}^{1}$, there is a smooth rational cubic curve $C \subset \mathbb{P}^{3}$ such that $L \cap C=\varnothing$, and the diagram

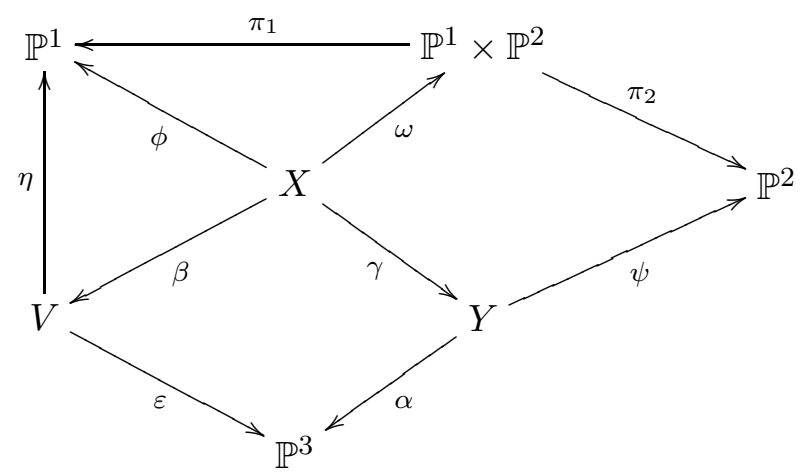

commutes, where $\alpha$ and $\beta$ are blow ups of the curve $C$ and its proper transform, respectively, the morphism $\gamma$ is a blow up of the proper transform of the line $L$, the morphism $\psi$ is a $\mathbb{P}^{1}$-bundle, the morphism $\omega$ is a birational contraction of a surface $F \subset X$ to a curve such that

$$
C \cup L \subset \alpha \circ \gamma(F) \subset \mathbb{P}^{3},
$$

and $\alpha \circ \gamma(F)$ consists of secant lines of $C \subset \mathbb{P}^{3}$ that intersect $L$, the morphism $\phi$ is a fibration into del Pezzo surfaces of degree 6, the morphisms $\pi_{1}$ and $\pi_{2}$ are natural projections.

Let $E$ and $G$ be exceptional divisors of $\beta$ and $\gamma$, respectively, let $Q \subset \mathbb{P}^{3}$ be a general quadric surface that passes through $C$, let $H \subset \mathbb{P}^{3}$ be a general plane that passes through $L$. Then

$$
-K_{X} \sim \bar{Q}+2 \bar{H}+G,
$$

where $\bar{Q} \subset X \supset \bar{H}$ are proper transforms of $Q \subset \mathbb{P}^{3} \supset H$, respectively. In particular, $\operatorname{lct}(X) \leqslant 1 / 2$.

We suppose that $\operatorname{lct}(X)<1 / 2$. Then there exists an effective $\mathbb{Q}$-divisor $D \sim_{\mathbb{Q}}-K_{X}$ such that the log pair $(X, \lambda D)$ is not log canonical for some positive rational number $\lambda<1 / 2$. Note that

$$
\varnothing \neq \operatorname{LCS}(X, \lambda D) \subset G,
$$

since $\operatorname{lct}(Y)=1 / 2$ by Lemma 8.13 . Applying Theorem 2.28 to $\phi$ we see that

$$
\varnothing \neq \operatorname{LCS}(X, \lambda D) \subset G \cap S_{\phi},
$$

where $S_{\phi}$ is a singular fiber of the del Pezzo fibration $\phi$ (see Example 1.18). Then we see that

$$
\varnothing \neq \operatorname{LCS}(X, \lambda D) \subset G \cap S_{\phi} \cap F,
$$

by applying Theorem 2.28 to the log pair $\left(\mathbb{P}^{1} \times \mathbb{P}^{2}, \lambda \omega(D)\right)$ and to the $\mathbb{P}^{1}$-bundle $\pi_{2}$.

Let $Z_{1} \cong \mathbb{P}^{1}$ be a section of the natural projection

$$
\mathbb{P}^{1} \times \mathbb{P}^{1} \cong G \longrightarrow L \cong \mathbb{P}^{1}
$$

such that $Z_{1} \cdot Z_{1}=0$, and let $Z_{2}$ a fiber of this projection. Then

$$
\left.F\right|_{G} \sim Z_{1}+3 Z_{2}
$$

and $\left.S_{\phi}\right|_{G} \sim Z_{1}$. The curve $F \cap G$ is irreducible. Thus, we see that

$$
\left|G \cap F \cap S_{\phi}\right|<+\infty,
$$

which implies that the set $\operatorname{LCS}(X, \lambda D)$ consists of a single point $P \in G$ by Theorem 2.7

The log pair $(V, \lambda \beta(D))$ is not log canonical. Since $\beta$ is an isomorphism on $X \backslash E$, we see that

$$
\beta(P) \in \operatorname{LCS}(V, \lambda \beta(D)) \subseteq \beta(P) \cup \beta(E),
$$

which implies that $\operatorname{LCS}(V, \lambda \beta(D))=\beta(P)$ by Theorem 2.7. Let $H \subset \mathbb{P}^{3}$ be a general plane. Then

$$
\operatorname{LCS}\left(V, \lambda \beta(D)+\frac{1}{2}\left(\tilde{H}_{1}+3 \tilde{H}\right)\right)=\beta(P) \cup \tilde{H},
$$


where $\tilde{H} \subset V \supset \tilde{H}_{1}$ are proper transforms of $H \subset \mathbb{P}^{3} \supset H_{1}$, respectively. But

$$
-K_{V} \sim \tilde{H}_{1}+3 \tilde{H} \sim \mathbb{Q} \beta(D),
$$

which contradicts Theorem 2.7, because $\lambda<1 / 2$.

Lemma 9.14. Suppose that $I(X)=3.14$. Then $\operatorname{lct}(X)=1 / 2$.

Proof. Let $P \in \mathbb{P}^{3}$ be a point, and let $\alpha: V_{7} \rightarrow \mathbb{P}^{3}$ be a blow up of the point $P$. Then there is a natural $\mathbb{P}^{1}$-bundle $\pi: V_{7} \rightarrow \mathbb{P}^{2}$.

Let $\zeta: Z \rightarrow \mathbb{P}(1,1,1,2)$ be a blow up of the singular point of $\mathbb{P}(1,1,1,2)$. Then

$$
Z \cong \mathbb{P}\left(\mathcal{O}_{\mathbb{P}^{2}} \oplus \mathcal{O}_{\mathbb{P}^{2}}(2)\right)
$$

and there is a natural $\mathbb{P}^{1}$-bundle $\phi: Z \rightarrow \mathbb{P}^{2}$.

There is a plane $\Pi \subset \mathbb{P}^{3}$ and a smooth cubic curve $C \subset \Pi$ such that $P \notin \Pi$ and the diagram

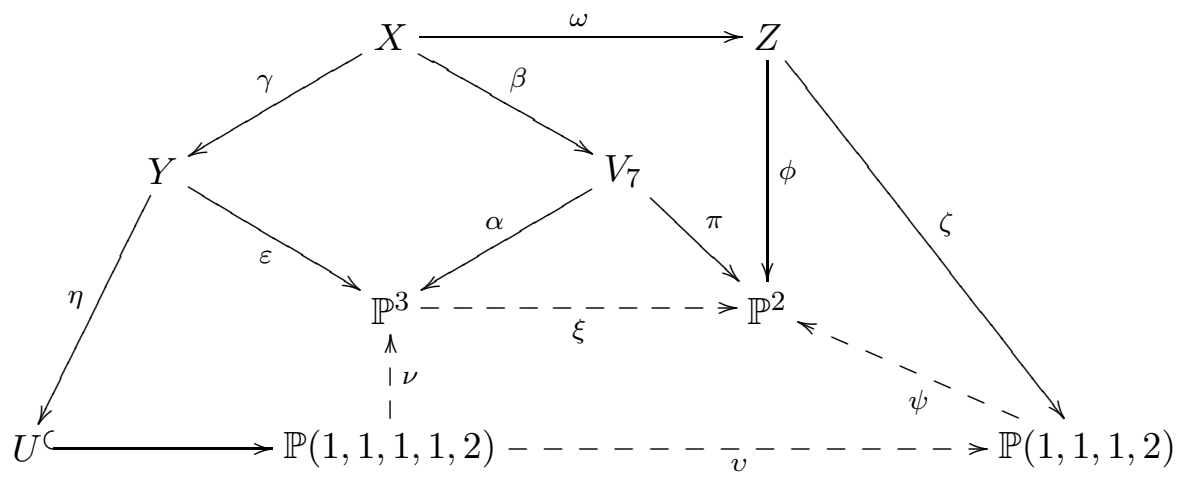

commutes (see [176, Example 3.6]), where we have the following notation:

- the morphism $\varepsilon$ is a blow up of the curve $C$;

- the threefold $U$ is a cubic hypersurface in $\mathbb{P}(1,1,1,1,2)$;

- the rational map $\xi$ is a projection from the point $P$;

- the morphism $\gamma$ is a blow up of the point that dominates $P$;

- the morphism $\beta$ is a blow up of the proper transform of the curve $C$;

- the morphism $\eta$ contracts the proper transform of $\Pi$ to the point $\operatorname{Sing}(U)$,

- the morphism $\omega$ contracts a surface $R \subset X$ to a curve such that

$$
\beta \circ \alpha(R) \subset \mathbb{P}^{3}
$$

is a cone over the curve $C$ whose vertex is the point $P$;

- the rational maps $\psi$ and $\nu$ are natural projections;

- the rational map $v$ is a linear projection from a point.

Let $E$ and $G$ be exceptional divisors of $\gamma$ and $\beta$, respectively, and let $\bar{H} \subset X$ be a proper transform of a general plane in $\mathbb{P}^{3}$ that passes through the point $P$. Then

$$
-K_{X} \sim \bar{\Pi}+3 \bar{H}+G,
$$

where $\bar{\Pi} \subset X$ is the proper transform of the plane $\Pi$. Thus, we see that $\operatorname{lct}(X) \leqslant 1 / 3$.

We suppose that $\operatorname{lct}(X)<1 / 3$. Then there exists an effective $\mathbb{Q}$-divisor $D \sim_{\mathbb{Q}}-K_{X}$ such that the log pair $(X, \lambda D)$ is not log canonical for some positive rational number $\lambda<1 / 3$.

Let $\bar{L} \subset X$ be a proper transform of a general line in $\mathbb{P}^{3}$ that intersects the curve $C$. Then

$$
D \cdot \bar{L}=\bar{\Pi} \cdot \bar{L}+3 \bar{H} \cdot \bar{L}+G \cdot \bar{L}=3 \bar{H} \cdot \bar{L}=3,
$$

which implies that $\mathbb{L} \mathbb{C} S(X, \lambda D)$ contains no surfaces except possibly $\bar{\Pi}$ and $E$.

Let $\Gamma$ be a general fiber of $\pi \circ \beta$. Then

$$
D \cdot \Gamma=\bar{\Pi} \cdot \Gamma+3 \bar{H} \cdot \Gamma+G \cdot \Gamma=\bar{\Pi} \cdot \Gamma+G \cdot \Gamma=2,
$$

which implies that $\mathbb{L} \mathbb{C} S(X, \lambda D)$ does not contain $\bar{\Pi}$ and $E$. Thus, by Lemma 2.9 , we have

$$
\varnothing \neq \operatorname{LCS}(X, \lambda D) \subsetneq E \cup G .
$$


Suppose that $\operatorname{LCS}(X, \lambda D) \subseteq E$. Then

$$
\varnothing \neq \operatorname{LCS}\left(V_{7}, \lambda \beta(D)\right) \subseteq \beta(E),
$$

which contradicts Theorem 2.28 , because $\beta(E)$ is a section of $\pi$. We see that $\operatorname{LCS}(X, \lambda D) \subsetneq G$.

Applying Theorem 2.28 to $(Z, \lambda \omega(D))$ and $\phi$ and Theorem 2.7 to $(X, \lambda D)$, we see that

$$
\varnothing \neq \operatorname{LCS}(X, \lambda D) \subseteq F,
$$

where $F$ is a fiber of the natural projection $G \rightarrow \beta(G)$. Then

$$
\varnothing \neq \operatorname{LCS}(Y, \lambda \gamma(D)) \subseteq \gamma(F),
$$

where $\gamma(F)$ is a fiber of the blow up $\varepsilon$ over a point in the curve $C$.

Let $S \subset \mathbb{P}^{3}$ be a general cone over the curve $C$, and let $O \in C$ be an inflection point such that

$$
\varepsilon \circ \gamma(F) \neq O \text {. }
$$

Let $L \subset S$ be a line that passes through the point $O$, and let $H \subset \mathbb{P}^{3}$ be a plane that is tangent to the cone $S$ along the line $L$. Since $O$ is an inflection point of the curve $C$, the equality

$$
\operatorname{mult}_{L}(S \cdot H)=3
$$

holds. Let $\breve{S}, \breve{H}$ and $\breve{L}$ be the proper transforms of $S, H$ and $L$ on the threefold $Y$. Then

$$
\operatorname{LCS}\left(Y, \lambda \gamma(D)+\frac{2}{3}(\breve{S}+\breve{H})\right)=\operatorname{LCS}(Y, \lambda \gamma(D)) \cup \breve{L}
$$

due to generality in the choice of $S$. But $-K_{Y} \sim \breve{S}+\breve{H}$, which is impossible by Theorem 2.7,

Lemma 9.15. Suppose that $I(X)=3.15$. Then $\operatorname{lct}(X)=1 / 2$.

Proof. Let $Q \subset \mathbb{P}^{4}$ be a smooth quadric hypersurface, let $C \subset Q$ be a smooth conic, and let $\varepsilon: V \rightarrow Q$ be a blow up of the conic $C \subset Q$. Then there is a natural morphism $\eta: V \rightarrow \mathbb{P}^{1}$ induced by the projection $Q \rightarrow \mathbb{P}^{1}$ from the two-dimensional linear subspace in $\mathbb{P}^{4}$ that contains the conic $C \subset Q$. Then a general fiber of $\eta$ is a smooth quadric surface in $\mathbb{P}^{3}$.

Take a line $L \subset Q$ such that $L \cap C=\varnothing$; then there is a commutative diagram

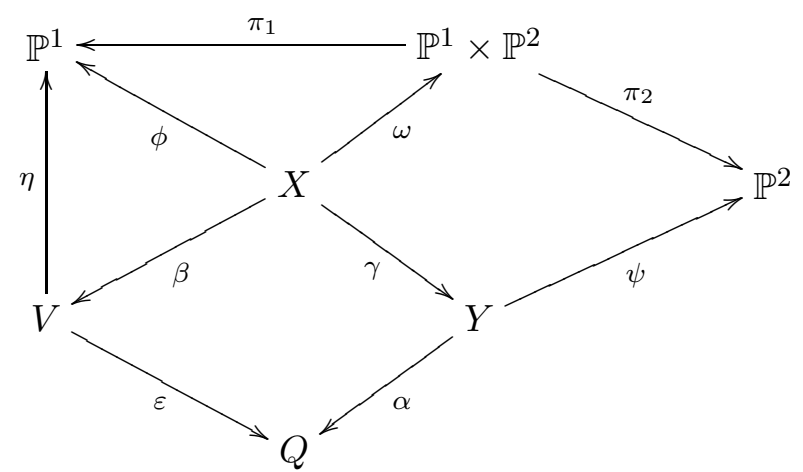

where $\alpha$ and $\beta$ are blow ups of the line $L \subset Q$ and its proper transform, respectively, the morphism $\gamma$ is a blow up of the proper transform of the conic $C$, the morphism $\psi$ is a $\mathbb{P}^{1}$-bundle, the morphism $\omega$ is a birational contraction of a surface $F \subset X$ to a curve such that

$$
C \cup L \subset \alpha \circ \gamma(F) \subset Q
$$

and $\alpha \circ \gamma(F)$ consists of all lines in $Q \subset \mathbb{P}^{4}$ that intersect $L$ and $C$, the morphism $\phi$ is a fibration into del Pezzo surfaces of degree 7 , the morphisms $\pi_{1}$ and $\pi_{2}$ are natural projections.

Let $E_{1}$ and $E_{2}$ be exceptional surfaces of $\beta$ and $\gamma$, respectively, let $H_{1}, H_{2} \subset Q$ be general hyperplane sections that pass through the curves $L$ and $C$, respectively. We have

$$
-K_{X} \sim \bar{H}_{1}+2 \bar{H}_{2}+E_{2} \sim \bar{H}_{2}+2 \bar{H}_{1}+E_{1},
$$

where $\bar{H}_{1} \subset X \supset \bar{H}_{2}$ are proper transforms of $H_{1} \subset Q \supset H_{2}$, respectively. In particular, $\operatorname{lct}(X) \leqslant 1 / 2$. 
We suppose that $\operatorname{lct}(X)<1 / 2$. Then there exists an effective $\mathbb{Q}$-divisor $D \sim_{\mathbb{Q}}-K_{X}$ such that the $\log$ pair $(X, \lambda D)$ is not $\log$ canonical for some positive rational number $\lambda<1 / 2$.

Let $S$ be an irreducible surface on the threefold $X$. Put

$$
D=\mu S+\Omega
$$

where $\Omega$ is an effective $\mathbb{Q}$-divisor such that $S \not \subset \operatorname{Supp}(\Omega)$. Then

$$
\operatorname{LCS}\left(\bar{H}_{2},\left.\frac{1}{2}(\mu S+\Omega)\right|_{\bar{H}_{2}}\right) \subset E_{1} \cap \bar{H}_{2}
$$

by Lemma 5.9. Thus, if $\mu \leqslant 2$, then either $S=E_{1}$, or $S$ is a fiber of $\phi$.

Let $\Gamma \cong \mathbb{P}^{1}$ be a general fiber of the conic bundle $\psi \circ \gamma$. Then

$$
2=D \cdot \Gamma=\mu S \cdot \Gamma+\Omega \cdot \Gamma \geqslant \mu S \cdot \Gamma,
$$

which implies that $\mu \leqslant 2$ in the case when either $S=E_{1}$, or $S$ is a fiber of $\phi$.

Therefore, we see that $\mathbb{L} \mathbb{C S}(X, \lambda D)$ does not contain surfaces.

Applying Theorem 2.28 to the $\log$ pair $(Y, \lambda \gamma(D))$ and $\psi$, we see that

$$
\varnothing \neq \operatorname{LCS}(X, \lambda D) \subsetneq E_{2} \cup \bar{L},
$$

where $\mathbb{P}^{1} \cong \bar{L} \subset X$ is a curve such that $\gamma(\bar{L})$ is a fiber of the conic bundle $\psi$.

Suppose that $\bar{L} \not \subset E_{1}$ and $\bar{L} \subset \operatorname{LCS}(X, \lambda D)$. Then

$$
\alpha \circ \gamma(\bar{L}) \subseteq \operatorname{LCS}(Q, \lambda \alpha \circ \gamma(D)) \subseteq \alpha \circ \gamma(\bar{L}) \cup C \cup L,
$$

which is impossible by Lemma 2.10. Hence by Theorem 2.7 we see that

- either $\operatorname{LCS}(X, \lambda D) \subsetneq E_{2}$,

- or $\operatorname{LCS}(X, \lambda D) \subseteq \bar{L}$ and $\bar{L} \subset E_{1}$.

We may assume that $\bar{L} \subset E_{1}$. Note that $E_{1} \cong \mathbb{F}_{1}$. One has $\bar{L} \cdot \bar{L}=-1$ on the surface $E_{1}$.

Applying Lemma 2.29 to the log pair $\left(\mathbb{P}^{1} \times \mathbb{P}^{2}, \lambda \omega(D)\right)$, we see that $\operatorname{LCS}(X, \lambda D) \subset F$, because

$$
\omega(D) \sim \mathbb{Q}_{\mathbb{P}}-K_{\mathbb{P}^{1} \times \mathbb{P}^{2}}
$$

and $\lambda<1 / 2$. Applying Lemma 2.26 to the $\log$ pair $(V, \lambda \beta(D))$ and the fibration $\eta$, we see that

$$
\varnothing \neq \operatorname{LCS}(X, \lambda D) \subsetneq E_{1} \cup S_{\phi},
$$

where $S_{\phi}$ is a singular fiber of $\phi$, because $\operatorname{lct}\left(\mathbb{P}^{1} \times \mathbb{P}^{1}\right)=1 / 2$ (see Example 1.18).

We have $F \cap \bar{L}=\varnothing$ and $\left|F \cap \bar{S}_{\phi} \cap E_{2}\right|<+\infty$. Thus, there is point $P \in E_{2}$ such that

$$
\operatorname{LCS}(X, \lambda D)=P \in E_{2}
$$

by Theorem 2.7. But $\beta\left(E_{1}\right) \cap \beta(P)=\varnothing$. Thus, it follows from Theorem 2.7 that

$$
\operatorname{LCS}(V, \lambda \beta(D))=\beta(P) .
$$

Let $\tilde{H}_{1} \subset V \supset \tilde{H}_{2}$ be the proper transforms of $H_{1} \subset Q \supset H_{2}$, respectively. Then

$$
-K_{V} \sim \tilde{H}_{2}+2 \tilde{H}_{1} \sim_{\mathbb{Q}} \beta(D)
$$

but it follows from the generality of $H_{1}$ and $H_{2}$ that

$$
\operatorname{LCS}\left(V, \lambda \beta(D)+\frac{1}{2}\left(\tilde{H}_{2}+2 \tilde{H}_{1}\right)\right)=\beta(P) \cup \tilde{H}_{1},
$$

which is impossible by Theorem 2.7, because $\lambda<1 / 2$.

Lemma 9.16. Suppose that $I(X)=3.16$. Then $\operatorname{lct}(X)=1 / 2$. 
Proof. Let $\mathbb{P}^{1} \cong C \subset \mathbb{P}^{3}$ be a twisted cubic curve, let $O \in C$ be a point. There is a commutative diagram

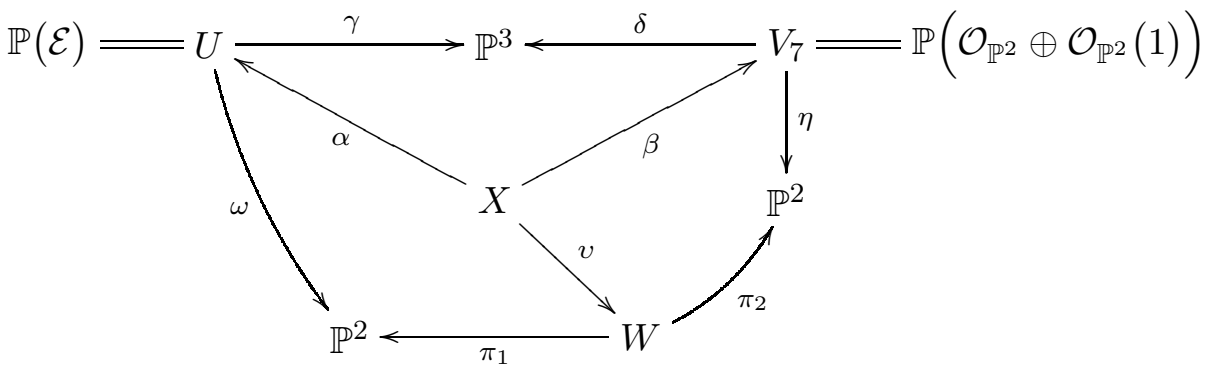

where $\mathcal{E}$ is a stable rank two vector bundle on $\mathbb{P}^{2}$ (see the proof of Lemma 8.13 ), and we have the following notation:

- the morphism $\delta$ is a blow up of the point $O$;

- the morphism $\gamma$ contracts a surface $G \subset U$ to the curve $C \subset \mathbb{P}^{3}$;

- the morphism $\alpha$ contracts a surface $E \cong \mathbb{F}_{1}$ to the fiber of $\gamma$ over the point $O \in \mathbb{P}^{3}$;

- the morphism $\beta$ is a blow up of the proper transform of the curve $C$;

- the variety $W$ is a smooth divisor on $\mathbb{P}^{2} \times \mathbb{P}^{2}$ of bi-degree $(1,1)$;

- the morphisms $\pi_{1}$ and $\pi_{2}$ are natural projections;

- the morphisms $\omega$ and $\eta$ are natural $\mathbb{P}^{1}$-bundles;

- the morphism $v$ contracts a surface $F \subset X$ to a curve

$$
\mathbb{P}^{1} \cong Z \subset W
$$

such that $\omega \circ \alpha(E)=\pi_{1}(Z)$ and $\eta \circ \beta(G)=\pi_{2}(Z)$.

Take general surfaces $H_{1} \in\left|(\omega \circ \alpha)^{*}\left(\mathcal{O}_{\mathbb{P}^{2}}(1)\right)\right|$ and $H_{2} \in\left|(\eta \circ \beta)^{*}\left(\mathcal{O}_{\mathbb{P}^{2}}(1)\right)\right|$. Then

$$
-K_{X} \sim H_{1}+2 H_{2}
$$

which implies that $\operatorname{lct}(X) \leqslant 1 / 2$.

We suppose that $\operatorname{lct}(X)<1 / 2$. Then there exists an effective $\mathbb{Q}$-divisor $D \sim_{\mathbb{Q}}-K_{X}$ such that the $\log$ pair $(X, \lambda D)$ is not log canonical for some positive rational number $\lambda<1 / 2$. Note that

$$
\varnothing \neq \operatorname{LCS}(X, \lambda D) \subseteq E \cap F,
$$

because $\operatorname{lct}(U)=1 / 2$ by Lemma 8.11 and $\operatorname{lct}(W)=1 / 2$ by Theorem 7.1 .

Applying Lemma 2.12 to the log pair $\left(V_{7}, \lambda \beta(D)\right)$, we see that

$$
\operatorname{LCS}(X, \lambda D)=E \cap F \cap G,
$$

where $|E \cap F \cap G|=1$. Then

$$
\operatorname{LCS}\left(X, \lambda D+H_{2}\right)=\operatorname{LCS}(X, \lambda D) \cup H_{2},
$$

where $H_{2} \cap \operatorname{LCS}(X, \lambda D)=\varnothing$. But the divisor

$$
-\left(K_{X}+\lambda D+H_{2}\right) \sim_{\mathbb{Q}}\left(\lambda-\frac{1}{2}\right) K_{X}+\frac{1}{2} H_{1}
$$

is ample, which is impossible by Theorem 2.7 .

Lemma 9.17. Suppose that $I(X)=3.17$. Then $\operatorname{lct}(X)=1 / 2$.

Proof. The threefold $X$ is a divisor in $\mathbb{P}^{1} \times \mathbb{P}^{1} \times \mathbb{P}^{2}$ of tri-degree $(1,1,1)$. Take general surfaces

$$
H_{1} \in\left|\pi_{1}^{*}\left(\mathcal{O}_{\mathbb{P}^{1}}(1)\right)\right|, H_{2} \in\left|\pi_{2}^{*}\left(\mathcal{O}_{\mathbb{P}^{1}}(1)\right)\right|, H_{3} \in\left|\pi_{3}^{*}\left(\mathcal{O}_{\mathbb{P}^{2}}(1)\right)\right|,
$$

where $\pi_{i}$ is a natural projection of the threefold $X$ onto the $i$-th factor of $\mathbb{P}^{1} \times \mathbb{P}^{1} \times \mathbb{P}^{2}$. Then

$$
-K_{X} \sim H_{1}+H_{2}+2 H_{3},
$$


which implies that $\operatorname{lct}(X) \leqslant 1 / 2$. There is a commutative diagram

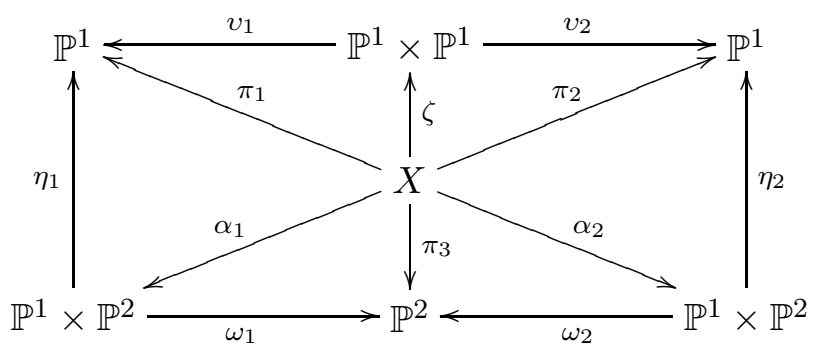

where $\omega_{i}, \eta_{i}$ and $v_{i}$ are natural projections, $\zeta$ is a $\mathbb{P}^{1}$-bundle, and $\alpha_{i}$ is a birational morphism that contracts a surface $E_{i} \subset X$ to a smooth curve $C_{i} \subset \mathbb{P}^{1} \times \mathbb{P}^{2}$ such that $\omega_{1}\left(C_{1}\right)=\omega_{2}\left(C_{2}\right)$ is a (irreducible) conic.

Note that $E_{2} \sim H_{1}+H_{3}-H_{2}$ and $E_{1} \sim H_{2}+H_{3}-H_{1}$.

We suppose that $\operatorname{lct}(X)<1 / 2$. Then there exists an effective $\mathbb{Q}$-divisor $D \sim_{\mathbb{Q}}-K_{X}$ such that the log pair $(X, \lambda D)$ is not log canonical for some positive rational number $\lambda<1 / 2$.

Suppose that the set $\mathbb{L} \mathbb{C S}(X, \lambda D)$ contains a (irreducible) surface $S \subset X$. Put

$$
D=\mu S+\Omega
$$

where $\mu \geqslant 1 / \lambda$ and $\Omega$ is an effective $\mathbb{Q}$-divisor such that $S \not \subset \operatorname{Supp}(\Omega)$. Then

$$
2=D \cdot \Gamma=\mu S \cdot \Gamma+\Omega \cdot \Gamma \geqslant \mu S \cdot \Gamma,
$$

where $\Gamma \cong \mathbb{P}^{1}$ is a general fiber of $\zeta$. Hence $S \cdot \Gamma=0$, which implies that $E_{2} \neq S \neq E_{1}$. One also has

$$
2=D \cdot \Delta=\mu S \cdot \Delta+\Omega \cdot \Delta \geqslant \mu S \cdot \Delta,
$$

where $\Delta \cong \mathbb{P}^{1}$ is a general fiber of the conic bundle $\pi_{2}$. Hence $S \cdot \Delta=0$, which implies that

$$
S \in\left|\pi_{3}^{*}\left(\mathcal{O}_{\mathbb{P}^{2}}(m)\right)\right|
$$

for some $m \in \mathbb{Z}_{>0}$, because $E_{2} \neq S \neq E_{1}$ and $S$ is an irreducible surface. Then

$$
0=S \cdot \Gamma=m \neq 0,
$$

which is a contradiction. Thus, we see that the set $\mathbb{L} \mathbb{C} S(X, \lambda D)$ contains no surfaces.

Applying Theorem 2.28 to $\zeta$ and using Theorem 2.7, we see that

$$
\operatorname{LCS}(X, \lambda D)=F \cong \mathbb{P}^{1},
$$

where $F$ is a fiber of the $\mathbb{P}^{1}$-bundle $\zeta$. Applying Theorem 2.28 to the conic bundle $\pi_{3}$, we see that every fiber of the conic bundle $\pi_{3}$ that intersects $F$ must be reducible. This means that

$$
\pi_{3}(F) \subset \omega_{1}\left(C_{1}\right)=\omega_{2}\left(C_{2}\right) \subset \mathbb{P}^{2},
$$

which is impossible, because $\pi_{3}(F)$ is a line, and $\omega_{1}\left(C_{1}\right)=\omega_{2}\left(C_{2}\right)$ is an irreducible conic.

Lemma 9.18. Suppose that $I(X)=3.18$. Then $\operatorname{lct}(X)=1 / 3$.

Proof. Let $Q \subset \mathbb{P}^{4}$ be a smooth quadric hypersurface, $C \subset Q$ an irreducible conic, and $O \in C$ a point. Then there is a commutative diagram

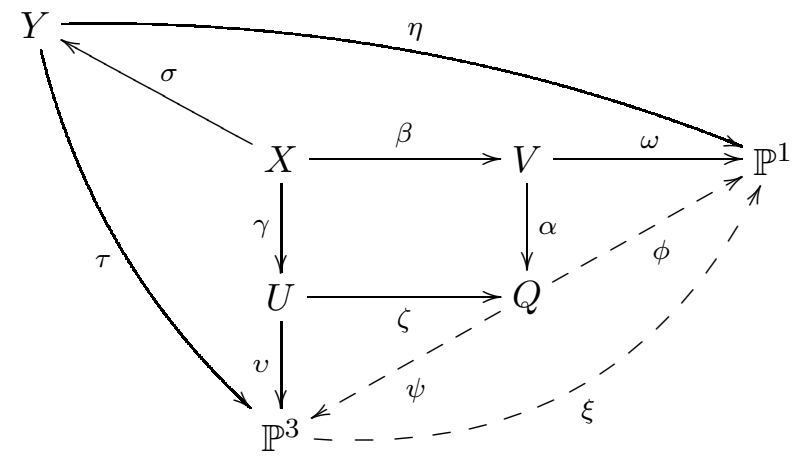


where $\zeta$ is a blow up of the point $O$, the morphisms $\alpha$ and $\gamma$ are blow ups of the conic $C$ and its proper transform, respectively, $\beta$ is a blow up of the fiber of the morphism $\alpha$ over the point $O$, the map $\psi$ is a projection from $O$, the map $\phi$ is induced by the projection from the two-dimensional linear subspace that contains the conic $C$, the morphism $\tau$ is a blow up of the line $\psi(C)$, the morphism $v$ is a blow up of an irreducible conic $Z \subset \mathbb{P}^{3}$ such that

$$
\psi(C) \cap Z \neq \varnothing,
$$

and $Z$ and $\psi(C)$ are not contained in one plane, the morphism $\sigma$ is a blow up of the proper transform of the conic $Z$, the map $\xi$ is a projection from $\psi(C)$, the morphism $\eta$ is a $\mathbb{P}^{1}$-bundle, and $\omega$ is a fibration into quadric surfaces.

Let $\bar{H}$ be a general fiber of $\omega \circ \beta$. Then $\bar{H}$ is a del Pezzo surface such that $K_{\bar{H}}^{2}=7$, and

$$
-K_{X} \sim 3 \bar{H}+2 E+G,
$$

where $G$ and $E$ are the exceptional divisors of $\beta$ and $\gamma$, respectively. In particular, $\operatorname{lct}(X) \leqslant 1 / 3$.

We suppose that $\operatorname{lct}(X)<1 / 3$. Then there exists an effective $\mathbb{Q}$-divisor $D \sim_{\mathbb{Q}}-K_{X}$ such that the log pair $(X, \lambda D)$ is not log canonical for some positive rational number $\lambda<1 / 3$. Note that

$$
\varnothing \neq \operatorname{LCS}(X, \lambda D) \subseteq G
$$

since $\operatorname{lct}(V)=1 / 3$ by Lemma 8.15 and $\beta(D) \sim_{\mathbb{Q}}-K_{V}$.

Applying Lemma 2.26 to the del Pezzo fibration $\omega \circ \beta$ and using Theorem 2.7, we see that there is a unique singular fiber $S$ of the fibration $\omega \circ \beta$ such that

$$
\varnothing \neq \operatorname{LCS}(X, \lambda D) \subseteq G \cap S,
$$

because the equality $\operatorname{lct}(\bar{H})=1 / 3$ holds (see Example 1.18).

Let $P \in G \cap S$ be an arbitrary point of the locus $\operatorname{LCS}(X, \lambda D)$. Put

$$
D=\mu S+\Omega,
$$

where $\Omega$ is an effective $\mathbb{Q}$-divisor such that $S \not \subset \operatorname{Supp}(\Omega)$. Then

$$
P \in \operatorname{LCS}\left(S,\left.\lambda \Omega\right|_{S}\right)
$$

by Theorem 2.20 ,

We can identify the surface $\beta(S)$ with an irreducible quadric cone in $\mathbb{P}^{3}$. Note that $G \cap S$ is an exceptional curve on $S$, so that there is a unique ruling of the cone $\beta(S)$ intersecting the curve $\beta(G)$. Let $L \subset S$ be a proper transform of this ruling. Then $L \cap G \neq \varnothing$ (moreover, $|L \cap G \cap S|=1$ ), while $L \cap E=\varnothing$. Hence $P=L \cap G$ by Lemma 5.10. We see that $\operatorname{LCS}(X, \lambda D)=P$. One has

$$
\bar{H} \cup P \subseteq \operatorname{LCS}\left(X, \lambda D+\bar{H}+\frac{2}{3} E\right) \subseteq \bar{H} \cup P \cup E,
$$

because $\bar{H}$ is a general fiber of the fibration $\omega \circ \beta$. Therefore, the locus

$$
\varnothing \neq \operatorname{LCS}\left(X, \lambda D+\bar{H}+\frac{2}{3} E\right) \subset X
$$

must be disconnected, because $P \notin \bar{H}$ and $P \notin E$. But

$$
-\left(K_{X}+\lambda D+\bar{H}+\frac{2}{3} E\right) \sim_{\mathbb{Q}} \bar{H}+\frac{2}{3}(E+G)+(\lambda-1 / 3) K_{X}
$$

is an ample divisor, which is impossible by Theorem 2.7 .

The proof of Lemma 9.18 implies the following corollary.

Corollary 9.19. Suppose that $\beth(X)=4.4$ or $\beth(X)=5.1$. Then $\operatorname{lct}(X)=1 / 3$.

Lemma 9.20. Suppose that $I(X)=3.19$. Then $\operatorname{lct}(X)=1 / 3$. 
Proof. Let $Q \subset \mathbb{P}^{4}$ be a smooth quadric, and let $L \subset \mathbb{P}^{4}$ be a line such that

$$
L \cap Q=P_{1} \cup P_{2},
$$

where $P_{1}$ and $P_{2}$ are different points. Let $\eta: Q \rightarrow \mathbb{P}^{2}$ be the projection from $L$. The diagram

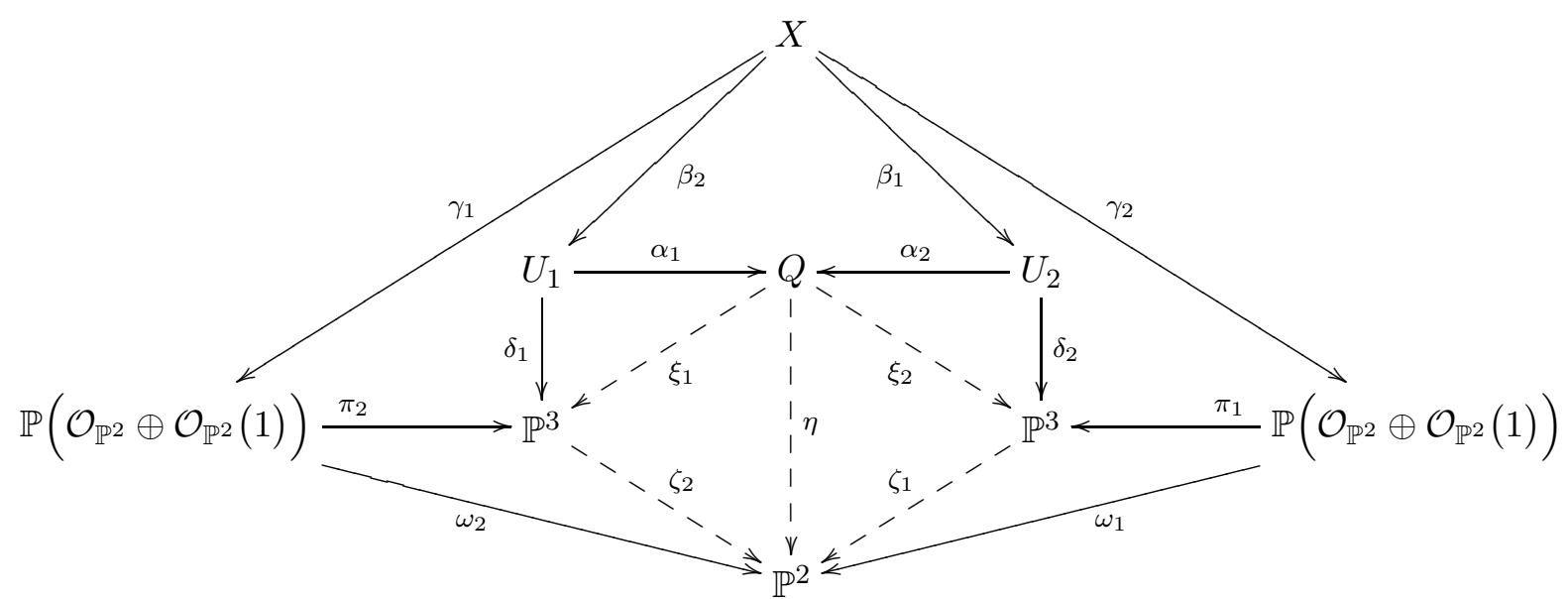

commutes, where $\alpha_{i}$ is a blow up of the point $P_{i}$, the morphism $\beta_{i}$ contracts a surface

$$
\mathbb{P}^{2} \cong E_{i} \subset X
$$

to the point that dominates $P_{i} \in Q$, the map $\xi_{i}$ is a projection from $P_{i}$, the map $\zeta_{i}$ is a projection from the image of $P_{i}$, the morphism $\delta_{i}$ is a contraction of a surface

$$
\mathbb{F}_{2} \cong G_{i} \subset U_{i}
$$

to a conic $C_{i} \subset \mathbb{P}^{3}$, the morphism $\pi_{i}$ is a blow up of the image of $P_{i}$, the morphism $\gamma_{i}$ contracts the proper transform of $G_{i}$ to the proper transform of $C_{i}$, and $\omega_{i}$ is a natural projection.

The map $\gamma_{1} \circ \gamma_{2}^{-1}$ is an elementary transformation of a conic bundle (see [166]), and

$$
\delta_{1} \circ \beta_{2}\left(E_{1}\right) \subset \mathbb{P}^{3} \supset \delta_{2} \circ \beta_{1}\left(E_{2}\right)
$$

are planes that contain the conics $C_{1}$ and $C_{2}$, respectively.

Let $H$ be a general hyperplane section of $Q$ such that $P_{1} \in H \ni P_{2}$. Then

$$
-K_{X} \sim 3 \bar{H}+E_{1}+E_{2},
$$

where $\bar{H}$ is the proper transform of $H$ on the threefold $X$. We see that $\operatorname{lct}(X) \leqslant 1 / 3$.

We suppose that $\operatorname{lct}(X)<1 / 3$. Then there exists an effective $\mathbb{Q}$-divisor $D \sim_{\mathbb{Q}}-K_{X}$ such that the log pair $(X, \lambda D)$ is not $\log$ canonical for some $\lambda<1 / 3$. Note that

$$
\varnothing \neq \operatorname{LCS}(X, \lambda D) \subseteq E_{1} \cup E_{2},
$$

because $\operatorname{lct}(Q)=1 / 3$. By Theorem 2.7, we may assume that

$$
\varnothing \neq \operatorname{LCS}(X, \lambda D) \subseteq E_{1} .
$$

Let $\bar{G}_{2} \subset X$ be a proper transform of $G_{2}$. Then $\bar{G}_{2} \cap E_{1}=\varnothing$, because $\alpha_{2}\left(G_{2}\right) \subset Q$ is a quadric cone whose vertex is the point $P_{2}$, and the line $L$ is not contained in $Q$. Hence

$$
\varnothing \neq \operatorname{LCS}\left(\mathbb{P}\left(\mathcal{O}_{\mathbb{P}^{2}} \oplus \mathcal{O}_{\mathbb{P}^{2}}(1)\right), \lambda \gamma_{2}(D)\right) \subseteq \gamma_{2}\left(E_{1}\right),
$$

where $\gamma_{2}\left(E_{1}\right)$ is a section of $\omega_{1}$. Applying Theorem 2.28 to $\omega_{1}$, we obtain a contradiction.

Lemma 9.21. Suppose that $I(X)=3.20$. Then $\operatorname{lct}(X)=1 / 3$.

Proof. Let $Q \subset \mathbb{P}^{4}$ be a smooth quadric threefold, and let

$$
W \subset \mathbb{P}^{2} \times \mathbb{P}^{2}
$$


be a smooth divisor of bi-degree $(1,1)$. Let $L_{1} \subset Q \supset L_{2}$ be lines such that $L_{1} \cap L_{2}=\varnothing$; then there exists a commutative diagram

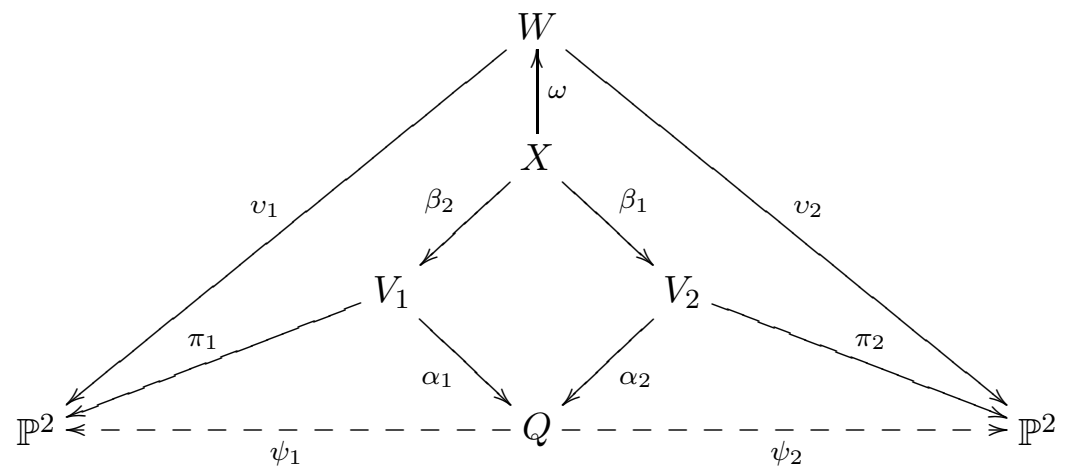

where the morphisms $\alpha_{i}$ and $\beta_{i}$ are blow ups of the line $L_{i}$ and its proper transform, respectively, the morphism $\omega$ is a blow up of a smooth curve $C \subset W$ of bi-degree $(1,1)$, the morphisms $v_{i}$ and $\pi_{i}$ are natural $\mathbb{P}^{1}$-bundles, and the map $\psi_{i}$ is a linear projection from the line $L_{i}$.

Let $\bar{H}$ be the exceptional divisor of $\omega$, and let $E_{i}$ be the exceptional divisor of $\beta_{i}$. Then

$$
-K_{X} \sim 3 \bar{H}+2 E_{1}+2 E_{2}
$$

because $\alpha_{2} \circ \beta_{1}(\bar{H}) \subset Q$ is a hyperplane section that contains $L_{1}$ and $L_{2}$. Hence $\operatorname{lct}(X) \leqslant 1 / 3$.

We suppose that $\operatorname{lct}(X)<1 / 3$. Then there exists an effective $\mathbb{Q}$-divisor $D \sim_{\mathbb{Q}}-K_{X}$ such that the log pair $(X, \lambda D)$ is not log canonical for some positive rational number $\lambda<1 / 3$. Note that

$$
\varnothing \neq \operatorname{LCS}(X, \lambda D) \subseteq E_{1} \cap E_{2} \cap \bar{H}=\varnothing,
$$

because $\operatorname{lct}\left(V_{1}\right)=\operatorname{lct}\left(V_{2}\right)=1 / 3$ by Lemma 8.17 and $\operatorname{lct}(W)=1 / 2$ by Theorem 7.1 , which gives a contradiction.

Lemma 9.22. Suppose that $I(X)=3.21$. Then $\operatorname{lct}(X)=1 / 3$.

Proof. Let $\pi_{1}: \mathbb{P}^{1} \times \mathbb{P}^{2} \rightarrow \mathbb{P}^{1}$ and $\pi_{2}: \mathbb{P}^{1} \times \mathbb{P}^{2} \rightarrow \mathbb{P}^{2}$ be natural projections. There is a morphism

$$
\alpha: X \longrightarrow \mathbb{P}^{1} \times \mathbb{P}^{2}
$$

that contracts a surface $E$ to a curve $C$ such that $\pi_{1}^{*}\left(\mathcal{O}_{\mathbb{P}^{1}}(1)\right) \cdot C=2$ and $\pi_{2}^{*}\left(\mathcal{O}_{\mathbb{P}^{2}}(1)\right) \cdot C=1$.

The curve $\pi_{2}(C) \subset \mathbb{P}^{2}$ is a line. Therefore, there is a unique surface

$$
H_{2} \in\left|\pi_{2}^{*}\left(\mathcal{O}_{\mathbb{P}^{2}}(1)\right)\right|
$$

such that $C \subset H_{2}$. Let $H_{1}$ be a fiber of the $\mathbb{P}^{2}$-bundle $\pi_{1}$. Then

$$
-K_{X} \sim 2 \bar{H}_{1}+3 \bar{H}_{2}+2 E,
$$

where $\bar{H}_{i} \subset X$ is a proper transform of the surface $H_{i}$. In particular, $\operatorname{lct}(X) \leqslant 1 / 3$.

We suppose that $\operatorname{lct}(X)<1 / 3$. Then there exists an effective $\mathbb{Q}$-divisor $D \sim_{\mathbb{Q}}-K_{X}$ such that the log pair $(X, \lambda D)$ is not $\log$ canonical for some rational $\lambda<1 / 3$. Note that

$$
\operatorname{LCS}(X, \lambda D) \subseteq E,
$$

because $\operatorname{lct}\left(\mathbb{P}^{1} \times \mathbb{P}^{2}\right)=1 / 3$ by Lemma 2.22 , There is a commutative diagram

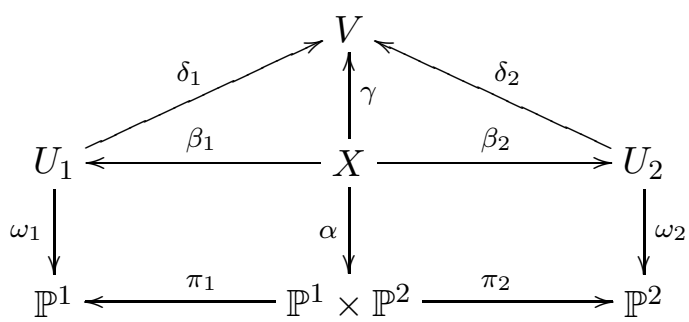

where $V$ is a Fano threefold of index 2 with one ordinary double point $O \in V$ such that $-K_{V}^{3}=40$, the birational morphism $\beta_{i}$ is a contraction of the surface $\bar{H}_{2} \cong \mathbb{P}^{1} \times \mathbb{P}^{1}$ to a smooth 
rational curve, the morphism $\delta_{i}$ contracts the curve $\beta_{i}\left(\bar{H}_{2}\right)$ to the point $O \in V$ such that the rational map

$$
\delta_{2} \circ \delta_{1}^{-1}: U_{1} \rightarrow U_{2}
$$

is a standard flop in $\beta_{1}\left(\bar{H}_{2}\right) \cong \mathbb{P}^{1}$, the morphism $\omega_{1}$ is a fibration whose general fiber is $\mathbb{P}^{1} \times \mathbb{P}^{1}$, the morphism $\omega_{2}$ is a $\mathbb{P}^{1}$-bundle, and $\gamma$ is a birational morphism such that $\gamma\left(\bar{H}_{2}\right)=O \in V$.

The variety $V$ is a section of $\operatorname{Gr}(2,5) \subset \mathbb{P}^{9}$ by a linear subspace of codimension 3 . One has

$$
-K_{V} \sim 2\left(\gamma\left(\bar{H}_{1}\right)+\gamma(E)\right),
$$

and the divisor $\gamma\left(\bar{H}_{1}\right)+\gamma(E)$ is very ample. There is a commutative diagram

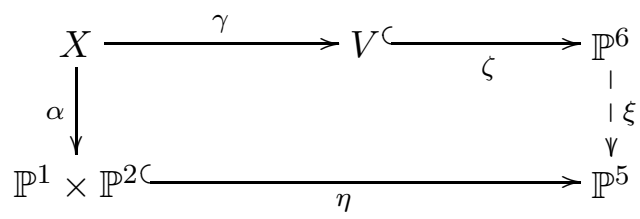

such that the embedding $\zeta$ is given by the linear system $\left|\gamma\left(\bar{H}_{1}\right)+\gamma(E)\right|$, the map $\xi$ is a linear projection from the point $O$, the embedding $\eta$ is given by the linear system $\left|H_{1}+H_{2}\right|$.

It follows from [85, Theorem 3.6] (see [86, Theorem 3.13]) that $U_{2} \cong \mathbb{P}(\mathcal{E})$, where where $\mathcal{E}$ is a stable rank two vector bundle on $\mathbb{P}^{2}$ such that the sequence

$$
0 \longrightarrow \mathcal{O}_{\mathbb{P}_{2}} \longrightarrow \mathcal{E} \otimes \mathcal{O}_{\mathbb{P}_{2}}(1) \longrightarrow \mathcal{I} \otimes \mathcal{O}_{\mathbb{P}_{2}}(1) \longrightarrow 0
$$

is exact, where $\mathcal{I}$ is an ideal sheaf of two general points on $\mathbb{P}^{2}$. One has $c_{1}(\mathcal{E})=-1$ and $c_{1}(\mathcal{E})=2$, and $\mathcal{E}$ is a Hulsbergen bundle (see [80]). It follows from [85, Theorem 3.5] that

$$
U_{1} \subset \mathbb{P}\left(\mathcal{O}_{\mathbb{P}^{1}} \oplus \mathcal{O}_{\mathbb{P}^{1}}(1) \oplus \mathcal{O}_{\mathbb{P}^{1}}(1) \oplus \mathcal{O}_{\mathbb{P}^{1}}(1)\right),
$$

and $U_{1} \in|2 T-F|$, where $T$ is a tautological bundle on $\mathbb{P}\left(\mathcal{O}_{\mathbb{P}^{1}} \oplus \mathcal{O}_{\mathbb{P}^{1}}(1) \oplus \mathcal{O}_{\mathbb{P}^{1}}(1) \oplus \mathcal{O}_{\mathbb{P}^{1}}(1)\right)$, and $F$ is a fiber of the projection $\mathbb{P}\left(\mathcal{O}_{\mathbb{P}^{1}} \oplus \mathcal{O}_{\mathbb{P}^{1}}(1) \oplus \mathcal{O}_{\mathbb{P}^{1}}(1) \oplus \mathcal{O}_{\mathbb{P}^{1}}(1)\right) \rightarrow \mathbb{P}^{1}$.

Either $\bar{H}_{1}$ is a smooth del Pezzo surface such that $K_{\bar{H}_{1}}^{2}=7$, or

$$
\left|H_{1} \cap C\right|=1,
$$

because $H_{1} \cdot C=2$. Applying Lemma 2.26 to the morphism $\omega_{1} \circ \beta_{1}$ and the surface $\bar{H}_{1}$, we see that

- either $\left|H_{1} \cap C\right|=1$,

- or $H_{1} \cap \operatorname{LCS}(X, \lambda D)=\varnothing$,

because $\operatorname{lct}\left(\bar{H}_{1}\right)=1 / 3$ if $\bar{H}_{1}$ is smooth. So, there is a fiber $L$ of the projection $E \rightarrow C$ such that

$$
\operatorname{LCS}(X, \lambda D) \subseteq L
$$

by Theorem 2.7, Put $\bar{C}=\bar{H}_{2} \cap E$ and $P=L \cap \bar{C}$. Applying Theorem 2.28 to $\omega_{2}$ and

$$
\left(U_{2}, \lambda \beta_{2}(D)\right)
$$

we see that either $\operatorname{LCS}(X, \lambda D)=P$ or $\operatorname{LCS}(X, \lambda D)=L$ by Theorem 2.7

Suppose that $\operatorname{LCS}(X, \lambda D)=L$. Then

$$
\operatorname{LCS}(V, \lambda \gamma(D))=\gamma(L) \subset V \subset \mathbb{P}^{6}
$$

where $\gamma(L) \subset V \subset \mathbb{P}^{6}$ is a line, because $-K_{V} \cdot \gamma(L)=2$ and $-K_{V} \sim_{\mathbb{Q}} \gamma(D)$. We have $\operatorname{Sing}(V)=O \in \gamma(L)$.

Let $S \subset V$ be a general hyperplane section of $V \subset \mathbb{P}^{6}$ such that $\gamma(L) \subset S$. Then

- the surface $S$ is a del Pezzo surface such that $K_{S}^{2}=5$,

- the point $O$ is an ordinary double point of the surface $S$,

- the surface $S$ is smooth outside of the point $O \in \gamma(L)$,

- the equivalence $\left.K_{S} \sim \mathcal{O}_{\mathbb{P}^{6}}(1)\right|_{S}$ holds, 
which implies that $S$ contains finitely many lines that intersect the line $\gamma(L)$.

Let $H \subset V$ be a general hyperplane section of $V \subset \mathbb{P}^{6}$. Put $Q=\gamma(L) \cap H$. Then

$$
\operatorname{LCS}\left(H,\left.\lambda \gamma(D)\right|_{H}\right)=Q
$$

by Remark 2.3, which contradicts Lemma 5.2, because $\lambda<1 / 3$.

Thus, we see that $\operatorname{LCS}(X, \lambda D)=P \in \bar{C}$. Let $F_{1}$ be a general fiber of $\pi_{1}$. Then

$$
F_{1} \cap C=P_{1} \cup P_{2} \not \supset \alpha(P),
$$

where $P_{1} \neq P_{2}$ are two points of the curve $C$. One has

$$
P_{1} \cup P_{2} \subset H_{2} \cap F_{1},
$$

because $C \subset H_{2}$. Let $Z$ be a general line in $F_{1} \cong \mathbb{P}^{2}$ such that $P_{1} \in Z$. Then there is a surface

$$
F_{2} \in\left|\pi_{2}^{*}\left(\mathcal{O}_{\mathbb{P}^{2}}(1)\right)\right|
$$

such that $Z \subset F_{2}$. Let $\bar{F}_{1} \subset X \supset \bar{F}_{2}$ be the proper transforms of $F_{1}$ and $F_{2}$, respectively. Then

$$
P \notin \bar{F}_{1} \cup \bar{F}_{2} \text {. }
$$

Let $\bar{Z} \subset X$ be the proper transform of the curve $Z$. Then $-K_{X} \cdot \bar{Z}=2$ and

$$
\bar{Z} \subset \bar{F}_{1} \cap \bar{F}_{2}
$$

but $\bar{Z} \cap \bar{H}_{2}=\varnothing$. Thus, the curve $\gamma(\bar{Z})$ is a line on $V \subset \mathbb{P}^{6}$ such that $\operatorname{Sing}(V)=O \notin \gamma(\bar{Z})$.

Let $T$ be a general hyperplane section of the threefold $V \subset \mathbb{P}^{6}$ such that $\gamma(\bar{Z}) \subset T$. Then

$$
\bar{T} \sim 2 \bar{H}_{2}+\bar{H}_{1}+E \sim 2 \bar{H}_{2}+\bar{F}_{1}+E \sim 2 \bar{F}_{2}+\bar{F}_{1}-E,
$$

where $\bar{T}$ is the proper transform of the surface $T$ on the threefold $X$. Hence

$$
\bar{F}_{1}+\bar{F}_{2}+\bar{T} \sim 3 \bar{F}_{2}+2 \bar{F}_{1}-E \sim 2 \bar{H}_{2}+2 \bar{H}_{1}+2 E \sim-K_{X},
$$

and applying Theorem 2.7, we see that the locus

$$
P \cup \bar{Z}=\operatorname{LCS}\left(X, \lambda D+\frac{2}{3}\left(\bar{F}_{1}+\bar{F}_{2}+\bar{T}\right)\right)
$$

must be connected. But $P \notin \bar{Z}$, which is a contradiction.

Lemma 9.23. Suppose that $I(X)=3.22$. Then $\operatorname{lct}(X)=1 / 3$.

Proof. Let $\pi_{1}: \mathbb{P}^{1} \times \mathbb{P}^{2} \rightarrow \mathbb{P}^{1}$ and $\pi_{2}: \mathbb{P}^{1} \times \mathbb{P}^{2} \rightarrow \mathbb{P}^{2}$ be natural projections. There is a morphism

$$
\alpha: X \longrightarrow \mathbb{P}^{1} \times \mathbb{P}^{2}
$$

that contracts a surface $E$ to a curve $C$ contained in a fiber $H_{1}$ of $\pi_{1}$ such that $\pi_{2}(C)$ is a conic.

We have $E \cong \mathbb{F}_{2}$. Let $H_{2}$ be a general surface in $\left|\pi_{2}^{*}\left(\mathcal{O}_{\mathbb{P}^{2}}(1)\right)\right|$. The equivalence

$$
-K_{X} \sim 2 \bar{H}_{1}+3 \bar{H}_{2}+E
$$

holds, where $\bar{H}_{i} \subset X$ is a proper transform of the surface $H_{i}$. Hence $\operatorname{lct}(X) \leqslant 1 / 3$.

We suppose that $\operatorname{lct}(X)<1 / 3$. Then there exists an effective $\mathbb{Q}$-divisor $D \sim_{\mathbb{Q}}-K_{X}$ such that the log pair $(X, \lambda D)$ is not $\log$ canonical for some rational $\lambda<1 / 3$. Note that

$$
\operatorname{LCS}(X, \lambda D) \subseteq E
$$

since $\operatorname{lct}\left(\mathbb{P}^{1} \times \mathbb{P}^{2}\right)=1 / 3$ by Lemma 2.22 .

Let $Q$ be the unique surface in $\left|\pi_{2}^{*}\left(\mathcal{O}_{\mathbb{P}^{2}}(2)\right)\right|$ such that $C \subset Q$, and let $\bar{Q} \subset X$ be the proper transform of the surface $Q$. Then $\bar{Q} \cap \bar{H}_{1}=\varnothing$, and there is a commutative diagram

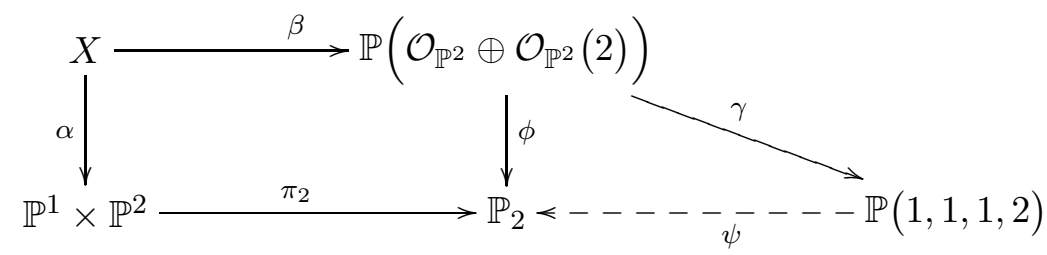


such that $\beta$ is a contraction of $\bar{Q}$ to a curve, $\gamma$ is a contraction of the surface $\beta\left(\bar{H}_{1}\right)$ to a point, the morphism $\phi$ is a natural $\mathbb{P}^{1}$-bundle, and the map $\psi$ is a natural projection. One has

$$
\gamma \circ \beta(D) \sim_{\mathbb{Q}} \frac{5 \gamma \circ \beta(E)}{2} \sim_{\mathbb{Q}}-K_{\mathbb{P}(1,1,1,2)} \sim_{\mathbb{Q}} \mathcal{O}_{\mathbb{P}(1,1,1,2)}(5)
$$

which implies that $E \nsubseteq \operatorname{LCS}(X, \lambda D)$, because $\lambda<1 / 3$.

Applying Theorem 2.28 to $\phi$, we see that there is a fiber $F$ of the projection $E \rightarrow C$ such that

$$
\varnothing \neq \operatorname{LCS}(X, \lambda D) \subseteq(E \cap \bar{Q}) \cup F,
$$

including the possibility that $\operatorname{LCS}(X, \lambda D) \subset E \cap \bar{Q}$.

Suppose that $\operatorname{LCS}(X, \lambda D) \subset E \cap \bar{Q}$. Let $M \subset \mathbb{P}^{1} \times \mathbb{P}^{2}$ be a general surface in $\left|H_{1}+H_{2}\right|$, and let $\bar{M} \subset X$ be the proper transform of the surface $M$. Then

$$
\bar{M} \cap \bar{H}_{1}=L,
$$

where $L$ is a line on $\bar{H}_{1} \cong \mathbb{P}^{2}$. Let $R$ be the unique surface in $\left|\pi_{2}^{*}\left(\mathcal{O}_{\mathbb{P}^{2}}(1)\right)\right|$ such that $\alpha(L) \subset R$, and let $\bar{R}$ be a proper transform of the surface $R$ on the threefold $X$. Then

$$
\operatorname{LCS}(X, \lambda D) \cup L \subseteq \operatorname{LCS}\left(X, \lambda D+\frac{2}{3}\left(\bar{M}+\bar{H}_{1}+\bar{R}+\bar{H}_{2}\right)\right) \subseteq \operatorname{LCS}(X, \lambda D) \cup L \cup \bar{H}_{1},
$$

but $L \cap E \cap \bar{Q}=\bar{Q} \cap \bar{H}_{1}=\varnothing$ and $-K_{X} \sim \bar{M}+\bar{H}_{1}+\bar{R}+\bar{H}_{2}$, which contradicts Theorem 2.7.

Therefore, we see that $F \subseteq \operatorname{LCS}(X, \lambda D)$. Put $\breve{F}=\gamma \circ \beta(F)$ and $\breve{D}=\gamma \circ \beta(D)$. Then

$$
\breve{F} \subseteq \operatorname{LCS}(\mathbb{P}(1,1,1,2), \lambda \breve{D}) \subseteq \breve{C} \cup \breve{F},
$$

where $\breve{C}=\gamma \circ \beta(\bar{Q}) \subset \mathbb{P}(1,1,1,2)$ is a curve such that $\psi(\breve{C})=\pi_{2}(C)$.

Let $S$ be a general surface in $\left|\mathcal{O}_{\mathbb{P}(1,1,1,2)}(2)\right|$. Then $S \cong \mathbb{P}^{2}$ and

$$
\breve{F} \cap S \subseteq \operatorname{LCS}\left(S,\left.\lambda \breve{D}\right|_{S}\right) \subseteq(\breve{C} \cup \breve{F}) \cap S
$$

but $\left.3 D\right|_{S} \sim_{\mathbb{Q}}-5 K_{S}$, which is impossible by Lemma 2.8.

Lemma 9.24. Suppose that $I(X)=3.23$. Then $\operatorname{lct}(X)=1 / 4$.

Proof. Let $O \in \mathbb{P}^{3}$ be a point, let $C \subset \mathbb{P}^{3}$ be a conic such that $O \in C$, let $\Pi \subset \mathbb{P}^{3}$ be a unique plane such that $C \subset \Pi$, and let $Q \subset \mathbb{P}^{4}$ be a smooth quadric threefold. Then the diagram

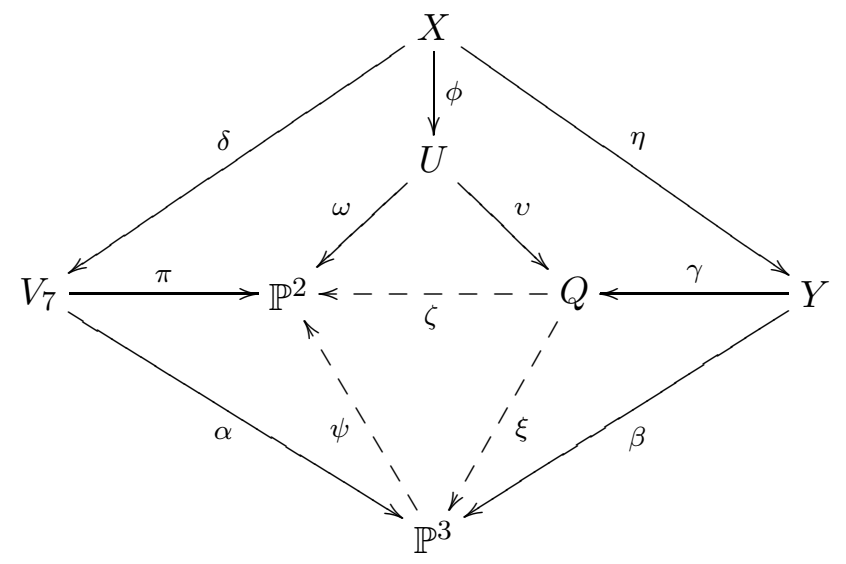

commutes, where we have the following notation:

- the morphism $\alpha$ is a blow up of the point $O$ with an exceptional divisor $E$;

- the morphism $\pi$ is a natural $\mathbb{P}^{1}$-bundle;

- the morphisms $\beta$ and $\delta$ are blow ups of $C$ and its proper transform, respectively;

- the morphism $\gamma$ contracts the proper transform of the plane $\Pi$ to a point;

- the morphism $\phi$ contracts the proper transform of the plane $\Pi$ to a curve;

- the morphism $\eta$ contracts the proper transform of $E$ to a curve $L \subset Y$ such that

$$
\gamma(\Pi) \in \gamma(L) \subset Q \subset \mathbb{P}^{4}
$$

and $\gamma(L)$ is a line in $\mathbb{P}^{4}$; 
- the morphism $\omega$ is a natural $\mathbb{P}^{1}$-bundle;

- the morphism $v$ is a blow up of the line $\gamma(L)$;

- the maps $\psi, \xi$ and $\zeta$ are projections from $O, \gamma(\Pi)$ and $\gamma(L)$, respectively.

Note that $E$ is a section of $\pi$.

Let $\bar{\Pi} \subset X$ be a proper transform of $\Pi \subset \mathbb{P}^{3}$. Then $\operatorname{lct}(X) \leqslant 1 / 4$, because

$$
-K_{X} \sim 4 \bar{\Pi}+2 \bar{E}+3 G,
$$

where $\bar{E}$ and $G$ are exceptional surfaces of $\eta$ and $\delta$, respectively.

We suppose that $\operatorname{lct}(X)<1 / 4$. Then there exists an effective $\mathbb{Q}$-divisor $D \sim_{\mathbb{Q}}-K_{X}$ such that the log pair $(X, \lambda D)$ is not log canonical for some positive rational number $\lambda<1 / 4$. Note that

$$
\varnothing \neq \operatorname{LCS}(X, \lambda D) \subseteq \bar{E} \cap \bar{\Pi} \cap G,
$$

because $\operatorname{lct}\left(V_{7}\right)=1 / 4$ by Lemma $[9.26, \operatorname{lct}(Y)=1 / 4$ by Lemma 8.16 and $\operatorname{lct}(U)=1 / 3$ by Lemma 8.17.

Let $R \subset \mathbb{P}^{3}$ be a general cone over $C$ whose vertex is $P \in \mathbb{P}^{3}$, let $H_{1} \subset \mathbb{P}^{3}$ be a general plane such that $O \in H_{1} \ni P$, and let $H_{2} \subset \mathbb{P}^{3}$ be a general plane such that $P \in H_{2}$. Then

$$
\bar{R} \sim(\alpha \circ \delta)^{*}(R)-\bar{E}-G, \bar{H}_{1} \sim(\alpha \circ \delta)^{*}\left(H_{1}\right)-\bar{E}, \bar{H}_{2} \sim(\alpha \circ \delta)^{*}\left(H_{2}\right),
$$

where $\bar{R}, \bar{H}_{1}, \bar{H}_{2}$ are proper transforms of $R, H_{1}, H_{2}$ on the threefold $X$, respectively. One has

$$
-K_{X} \sim \bar{Q}+\bar{H}_{1}+\bar{H}_{2},
$$

but it follows from the generality of $R, H_{1}, H_{2}$ that the locus

$$
\operatorname{LCS}\left(X, \lambda D+\frac{3}{4}\left(\bar{Q}+\bar{H}_{1}+\bar{H}_{2}\right)\right)=\operatorname{LCS}(X, \lambda D) \cup P,
$$

is disconnected, which is impossible by Theorem 2.7 .

Lemma 9.25. Suppose that $I(X)=3.24$. Then $\operatorname{lct}(X)=1 / 3$.

Proof. Let $W$ is a divisor of bi-degree $(1,1)$ on $\mathbb{P}^{2} \times \mathbb{P}^{2}$. There is a commutative diagram

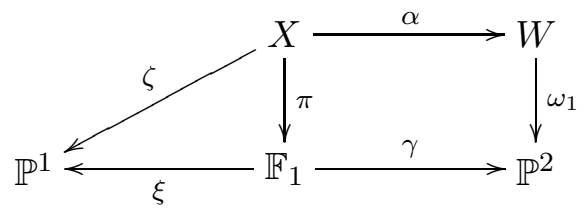

where $\omega_{1}$ is a natural $\mathbb{P}^{1}$-bundle, the morphism $\alpha$ contracts a smooth surface

$$
E \cong \mathbb{P}^{1} \times \mathbb{P}^{1}
$$

to a fiber $L$ of $\omega_{1}, \gamma$ is a blow up of the point $\omega_{1}(L)$, the morphism $\xi$ is a $\mathbb{P}^{1}$-bundle, and $\zeta$ is a $\mathbb{F}_{1}$-bundle.

Let $\omega_{2}: X \rightarrow \mathbb{P}^{2}$ be a natural $\mathbb{P}^{1}$-bundle that is different from $\omega_{1}$. Then there is a surface

$$
G \in\left|\omega_{2}^{*}\left(\mathcal{O}_{\mathbb{P}^{2}}(1)\right)\right|
$$

such that $L \subset G$, because $\omega_{2}(L)$ is a line. Let $\bar{G} \subset X$ be a proper transform of $G$. Then

$$
-K_{X} \sim 2 F+2 \bar{G}+3 E
$$

where $E$ is the exceptional divisor of $\alpha$, and $F$ is a fiber of $\zeta$. We see that $\operatorname{lct}(X) \leqslant 1 / 3$.

We suppose that $\operatorname{lct}(X)<1 / 3$. Then there exists an effective $\mathbb{Q}$-divisor $D \sim_{\mathbb{Q}}-K_{X}$ such that the log pair $(X, \lambda D)$ is not log canonical for some positive rational number $\lambda<1 / 3$. Note that

$$
\varnothing \neq \operatorname{LCS}(X, \lambda D) \subseteq E
$$

since $\operatorname{lct}(W)=1 / 2$ by Theorem [7.1, We may assume that $F \cap \operatorname{LCS}(X, \lambda D) \neq \varnothing$. Then

$$
\mathbb{F}_{1} \cong F \subseteq \operatorname{LCS}(X, \lambda D) \subseteq E \cong \mathbb{P}^{1} \times \mathbb{P}^{1}
$$

by Lemma 2.26, because lct $(F)=1 / 3$ (see Example 1.18), which is a contradiction. 
Lemma 9.26. Suppose that $I(X)=3.25$. Then $\operatorname{lct}(X)=1 / 3$.

Proof. Let $L_{1} \subset \mathbb{P}^{3} \supset L_{2}$ be lines such that $L_{1} \cap L_{2}=\varnothing$. Then there is a commutative diagram

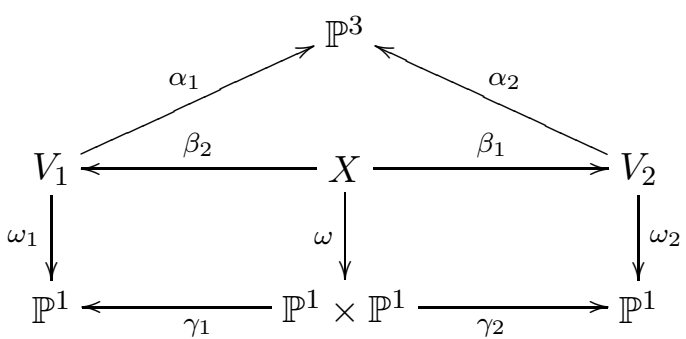

where the morphisms $\alpha_{i}$ and $\beta_{i}$ are blow ups of the line $L_{i}$ and its proper transform, respectively, the morphism $\omega_{i}$ is a natural $\mathbb{P}^{2}$-bundle, the morphisms $\omega$ and $\gamma_{i}$ are $\mathbb{P}^{1}$-bundles. Note that

$$
V_{1} \cong V_{2} \cong \mathbb{P}\left(\mathcal{O}_{\mathbb{P}^{1}} \oplus \mathcal{O}_{\mathbb{P}^{1}} \oplus \mathcal{O}_{\mathbb{P}^{1}}(1)\right)
$$

Let $H_{1}$ and $H_{2}$ be proper transforms on $X$ of planes in $\mathbb{P}^{3}$ such that $L_{i} \subset \alpha\left(H_{i}\right)$. Then

$$
-K_{X} \sim 2 H_{1}+2 H_{2}+E_{1}+E_{2} \sim 3 H_{1}+H_{2}+2 E_{1} \sim H_{1}+3 H_{2}+2 E_{2},
$$

where $E_{i}$ is an exceptional divisors of $\beta_{i}$. Hence $\operatorname{lct}(X) \leqslant 1 / 3$.

We suppose that $\operatorname{lct}(X)<1 / 3$. Then there exists an effective $\mathbb{Q}$-divisor $D \sim_{\mathbb{Q}}-K_{X}$ such that the log pair $(X, \lambda D)$ is not log canonical for some positive rational number $\lambda<1 / 3$.

Applying Lemma 2.26 to the $\mathbb{F}_{1}$-fibrations $\omega_{2} \circ \beta_{1}$ and $\omega_{1} \circ \beta_{2}$, we obtain a contradiction, because the equality $\operatorname{lct}\left(\mathbb{F}_{1}\right)=1 / 3$ holds (see Example 1.18).

Remark 9.27. Actually, the result of Lemma 9.26 is contained in Corollary 6.4, but we still prefer to give a detailed proof that may have further applications.

Lemma 9.28. Suppose that $I(X)=3.30$. Then $\operatorname{lct}(X)=1 / 4$.

Proof. Let $O \in \mathbb{P}^{3}$ be a point, and let $\gamma: V_{7} \rightarrow \mathbb{P}^{3}$ be a blow up of the point $O$. Then there is a $\mathbb{P}^{1}$-bundle $\pi: V_{7} \rightarrow \mathbb{P}^{1}$. Take a line $O \in L \subset \mathbb{P}^{3}$; then the diagram

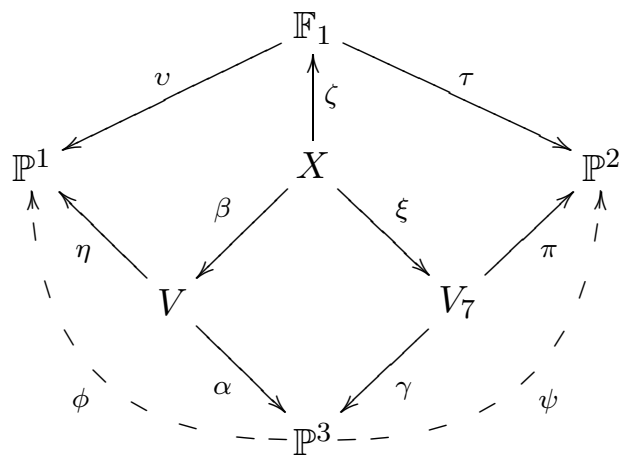

commutes, where $\alpha$ and $\xi$ are blow ups of the line $L$ and its proper transforms, respectively, the morphism $\eta$ is is a natural $\mathbb{P}^{2}$-bundle, the morphism $\beta$ is a blow up of the curve

$$
\mathbb{P}^{1} \cong C \subset V \cong \mathbb{P}\left(\mathcal{O}_{\mathbb{P}^{1}} \oplus \mathcal{O}_{\mathbb{P}^{1}} \oplus \mathcal{O}_{\mathbb{P}^{1}}(1)\right)
$$

such that $\beta(C)=O$, the morphisms $\zeta$ and $v$ are $\mathbb{P}^{1}$-bundles, the maps $\phi$ and $\psi$ are linear projections from $L$ and $O$, respectively, and $\tau$ is a blow up of the point $\psi(L) \in \mathbb{P}^{2}$.

Let $T$ be the proper transform on $X$ of a general plane in $\mathbb{P}^{3}$ that passes through $L \subset \mathbb{P}^{3}$, and let $G$ be the exceptional divisor of the blow up $\beta$. Then

$$
-K_{X} \sim 4 T+3 E+2 G,
$$

where $E$ is the proper transform on $X$ of the exceptional divisor of $\alpha$. In particular, $\operatorname{lct}(X)<1 / 4$.

We suppose that $\operatorname{lct}(X)<1 / 4$. Then there exists an effective $\mathbb{Q}$-divisor $D \sim_{\mathbb{Q}}-K_{X}$ such that the log pair $(X, \lambda D)$ is not log canonical for some rational number $\lambda<1 / 4$. Note that

$$
\varnothing \neq \operatorname{LCS}(X, \lambda D) \subseteq G
$$


because $\operatorname{lct}(V)=1 / 4$ by Lemma 7.2. However, every fiber of the morphism $\eta \circ \beta$ is isomorphic to $\mathbb{F}_{1}$, which is impossible by Lemma 2.26 , because lct $\left(\mathbb{F}_{1}\right)=1 / 3$ by Example 1.18 .

The proof of Lemma 9.28 implies the following corollary (cf. [176, Example 3.3]).

Corollary 9.29. Suppose that $I(X)=4.12$. Then $\operatorname{lct}(X)=1 / 4$.

Remark 9.30. Actually, the results of Lemma 9.28 and Corollary 9.29 are contained in Corollary 6.4, but we still prefer to give a detailed proof that may have further applications.

\section{FANO ThreEFolds With $\rho \geqslant 4$}

We use the assumptions and notation introduced in section 1.

Lemma 10.1. Suppose that $I(X)=4.1$. Then $\operatorname{lct}(X)=1 / 2$.

Proof. The threefold $X$ is a divisor on $\mathbb{P}^{1} \times \mathbb{P}^{1} \times \mathbb{P}^{1} \times \mathbb{P}^{1}$ of multidegree $(1,1,1,1)$. Let

$$
\left[\left(x_{1}: y_{1}\right),\left(x_{2}: y_{2}\right),\left(x_{3}: y_{3}\right),\left(x_{4}: y_{4}\right)\right]
$$

be coordinates on $\mathbb{P}^{1} \times \mathbb{P}^{1} \times \mathbb{P}^{1} \times \mathbb{P}^{1}$. Then $X$ is given by the equation

$$
F\left(x_{1}, y_{1}, x_{2}, y_{2}, x_{3}, y_{3}, x_{4}, y_{4}\right)=0,
$$

where $F$ is a of multidegree $(1,1,1,1)$.

Let $\pi_{1}: X \rightarrow \mathbb{P}^{1} \times \mathbb{P}^{1} \times \mathbb{P}^{1}$ be a projection given by

$$
\left[\left(x_{1}: y_{1}\right),\left(x_{2}: y_{2}\right),\left(x_{3}: y_{3}\right),\left(x_{4}: y_{4}\right)\right] \mapsto\left[\left(x_{2}: y_{2}\right),\left(x_{3}: y_{3}\right),\left(x_{4}: y_{4}\right)\right] \in \mathbb{P}^{1} \times \mathbb{P}^{1} \times \mathbb{P}^{1},
$$

and let $\pi_{2}, \pi_{3}$ and $\pi_{4}: X \rightarrow \mathbb{P}^{1} \times \mathbb{P}^{1} \times \mathbb{P}^{1}$ be projections defined in a similar way. Put

$$
F=x_{1} G\left(x_{2}, y_{2}, x_{3}, y_{3}, x_{4}, y_{4}\right)+y_{1} H\left(x_{2}, y_{2}, x_{3}, y_{3}, x_{4}, y_{4}\right)
$$

where $G\left(x_{2}, y_{2}, x_{3}, y_{3}, x_{4}, y_{4}\right)$ and $H\left(x_{2}, y_{2}, x_{3}, y_{3}, x_{4}, y_{4}\right)$ are multi-linear forms that do not depend on $x_{1}$ and $y_{1}$. Then $\pi_{1}$ is a blow up of a curve $C_{1} \subset \mathbb{P}^{1} \times \mathbb{P}^{1} \times \mathbb{P}^{1}$ given by the equations

$$
G\left(x_{2}, y_{2}, x_{3}, y_{3}, x_{4}, y_{4}\right)=H\left(x_{2}, y_{2}, x_{3}, y_{3}, x_{4}, y_{4}\right)=0
$$

which define a surface $E_{1} \subset \mathbb{P}^{1} \times \mathbb{P}^{1} \times \mathbb{P}^{1} \times \mathbb{P}^{1}$ that is contracted by $\pi_{1}$. The equations

$$
x_{1}=H\left(x_{2}, y_{2}, x_{3}, y_{3}, x_{4}, y_{4}\right)=0
$$

define a divisor $H_{1} \subset X$ such that $-K_{X} \sim 2 H_{1}+E_{1}$, which implies that $\operatorname{lct}(X) \leqslant 1 / 2$.

We suppose that $\operatorname{lct}(X)<1 / 2$. Then there exists an effective $\mathbb{Q}$-divisor $D \sim_{\mathbb{Q}}-K_{X}$ such that the log pair $(X, \lambda D)$ is not log canonical for some positive rational number $\lambda<1 / 2$.

Let $E_{2}, E_{3}, E_{4}$ be surfaces in $X$ defined in a way similar to $E_{1}$. Then

$$
\varnothing \neq \operatorname{LCS}(X, \lambda D) \subseteq E_{1} \cap E_{2} \cap E_{3} \cap E_{4},
$$

because $\operatorname{lct}\left(\mathbb{P}^{1} \times \mathbb{P}^{1} \times \mathbb{P}^{1}\right)=1 / 2$ by Lemma 2.22. But $E_{i} \subset \mathbb{P}^{1} \times \mathbb{P}^{1} \times \mathbb{P}^{1} \times \mathbb{P}^{1}$ is given by

$$
\frac{\partial F\left(x_{1}, y_{1}, x_{2}, y_{2}, x_{3}, y_{3}, x_{4}, y_{4}\right)}{\partial x_{i}}=\frac{\partial F\left(x_{1}, y_{1}, x_{2}, y_{2}, x_{3}, y_{3}, x_{4}, y_{4}\right)}{\partial y_{i}}=0,
$$

which implies that the intersection $E_{1} \cap E_{2} \cap E_{3} \cap E_{4}$ is given by the equations

$$
\frac{\partial F}{\partial x_{1}}=\frac{\partial F}{\partial y_{1}}=\frac{\partial F}{\partial x_{2}}=\frac{\partial F}{\partial y_{2}}=\frac{\partial F}{\partial x_{3}}=\frac{\partial F}{\partial y_{3}}=\frac{\partial F}{\partial x_{4}}=\frac{\partial F}{\partial y_{4}}=0 .
$$

Hence $E_{1} \cap E_{2} \cap E_{3} \cap E_{4}=\operatorname{Sing}(X)=\varnothing$, and $\operatorname{LCS}(X, \lambda D)=\varnothing$.

Lemma 10.2. Suppose that $I(X)=4.2$. Then $\operatorname{lct}(X)=1 / 2$. 
Proof. Let $Q_{1} \subset \mathbb{P}^{4} \supset Q_{2}$ be quadric cones, whose vertices are $O_{1} \in \mathbb{P}^{4} \ni O_{2}$, respectively. Let

$$
O_{1} \notin S_{1} \subset Q_{1} \subset \mathbb{P}^{4}
$$

be a hyperplane section of $Q_{1}$, and let $C_{1} \subset\left|-K_{S_{1}}\right|$ be a smooth elliptic curve. Then the diagram

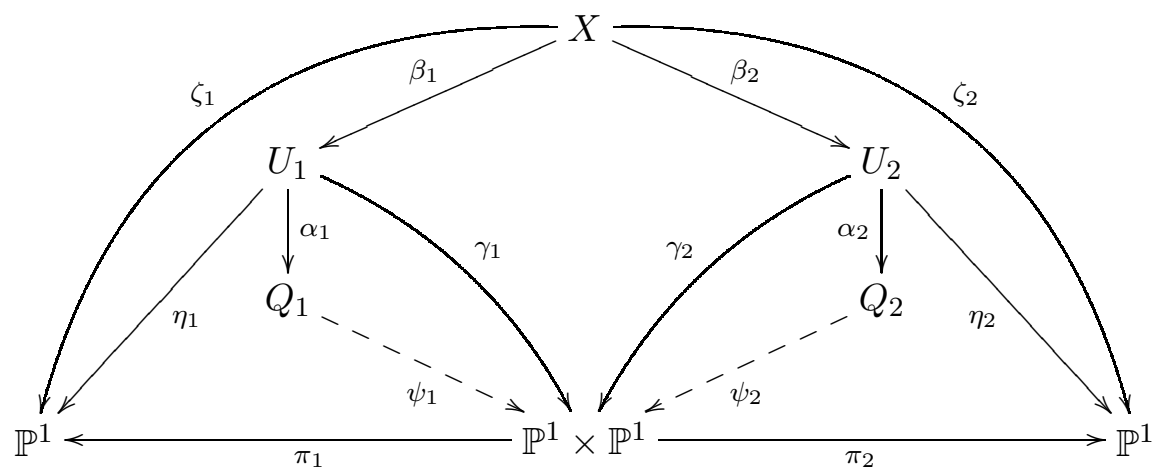

commutes, where $\pi_{1} \neq \pi_{2}$ are natural projections, the map $\psi_{i}$ is a projection from $O_{i} \in Q_{i} \subset \mathbb{P}^{4}$, the morphism $\alpha_{i}$ is a blow up of the vertex $O_{i}$, the morphism $\beta_{i}$ contracts a surface

$$
\mathbb{P}^{1} \times C_{1} \cong G_{i} \subset X
$$

to a curve $C_{1} \cong C_{i} \subset U_{i}$, the morphism $\eta_{i}$ is an $\mathbb{F}_{1}$-bundle, $\gamma_{i}$ is a $\mathbb{P}^{1}$-bundle, and $\zeta_{i}$ is a fibration into del Pezzo surfaces of degree 6 that has 4 singular fibers.

Let $E_{i} \subset X$ be the proper transform of the exceptional divisor of $\alpha_{i}$. Then

$$
S_{1}=\alpha_{1} \circ \beta_{1}\left(E_{2}\right) \subset Q_{1} \subset \mathbb{P}^{4} \supset Q_{2} \supset \alpha_{2} \circ \beta_{2}\left(E_{1}\right)
$$

are hyperplane sections that contain $C_{1}$ and $C_{2}$, respectively. It is also easy to see that

$$
\alpha_{1} \circ \beta_{1}\left(G_{2}\right) \subset Q_{1} \subset \mathbb{P}^{4} \supset Q_{2} \supset \alpha_{2} \circ \beta_{2}\left(G_{1}\right)
$$

are the cones in $\mathbb{P}^{4}$ over the curves $C_{1}$ and $C_{2}$, respectively.

Let $\bar{H} \subset X$ be the proper transform of a hyperplane section of $Q_{1} \subset \mathbb{P}^{4}$ that contains $O_{1}$. Then

$$
-K_{X} \sim 2 \bar{H}+E_{2}+E_{1},
$$

which gives $\operatorname{lct}(X) \leqslant 1 / 2$. Suppose that $\operatorname{lct}(X)<1 / 2$. Then there is an effective $\mathbb{Q}$-divisor

$$
D \sim_{\mathbb{Q}}-K_{X} \sim E_{1}+E_{2}+G_{1}+G_{2}
$$

such that the log pair $(X, \lambda D)$ is not $\log$ canonical for some $\lambda<1 / 2$. Put

$$
D=\mu_{1} E_{1}+\mu_{2} E_{2}+\Omega,
$$

where $\Omega$ is an effective $\mathbb{Q}$-divisor on $X$ such that

$$
E_{1} \not \subset \operatorname{Supp}(\Omega) \nsupseteq E_{2} \text {. }
$$

Let $\Gamma$ be a general fiber of the conic bundle $\gamma_{1} \circ \beta_{1}$. Then

$$
2=\Gamma \cdot D=\Gamma \cdot\left(\mu_{1} E_{1}+\mu_{2} E_{2}+\Omega\right)=\mu_{1}+\mu_{2}+\Gamma \cdot \Omega \geqslant \mu_{1}+\mu_{2},
$$

and without loss of generality we may assume that $\mu_{1} \leqslant \mu_{2}$. Then $\mu_{1} \leqslant 1$.

Suppose that there is a surface $S \in \mathbb{L} \mathbb{C S}(X, \lambda D)$. Then $S \neq E_{1}$. Moreover, we have $S \neq G_{1}$, because $\alpha_{2} \circ \beta_{2}\left(G_{1}\right)$ is a quadric surface and $\lambda<1 / 2$. Hence $S \cap E_{1} \neq \varnothing$. But

$$
-\left.\frac{1}{2} K_{E_{1}} \sim_{\mathbb{Q}} D\right|_{E_{1}} \sim_{\mathbb{Q}}-\frac{\mu_{1}}{2} K_{E_{1}}+\left.\Omega\right|_{E_{1}},
$$

and $E_{1} \cong \mathbb{P}^{1} \times \mathbb{P}^{1}$, which is impossible by Theorem 2.20 and Lemma 2.24,

We see that the set $\mathbb{L} \mathbb{C S}(X, \lambda D)$ contains no surfaces. Let $P \in \operatorname{LCS}(X, \lambda D)$ be a point.

Suppose that $P \notin G_{1}$. Let $Z$ be a fiber of $\gamma_{1}$ such that $\beta_{1}(P) \in Z$. Then

$$
Z \subseteq \operatorname{LCS}\left(U_{1}, \lambda \beta_{1}(D)\right)
$$

by Theorem 2.28, Put $\bar{E}_{1}=\beta_{1}\left(E_{1}\right)$. Then we have

$$
Z \cap \bar{E}_{1} \in \operatorname{LCS}\left(\bar{E}_{1},\left.\lambda \Omega\right|_{\bar{E}_{1}}\right)
$$


by Theorem 2.20, which is impossible by Lemma 2.24, because $\mu_{1} \leqslant 1$.

Thus, we see that $P \in G_{1}$. Let $F_{1} \subset X \supset F_{2}$ be fibers of $\zeta_{1}$ and $\zeta_{2}$ passing through the point $P$. Then either $F_{1}$ or $F_{2}$ is smooth, because $\alpha_{1}(P) \in C_{1}$. But

$$
\operatorname{lct}\left(F_{i}\right)=1 / 2
$$

in the case when $F_{i}$ is smooth (see Example 1.18), which contradicts Lemma 2.26.

Lemma 10.3. Suppose that $I(X)=4.3$. Then $\operatorname{lct}(X)=1 / 2$.

Proof. Let $F_{1} \cong F_{2} \cong F_{3} \cong \mathbb{P}^{1} \times \mathbb{P}^{1}$ be fibers of three different projections

$$
\mathbb{P}^{1} \times \mathbb{P}^{1} \times \mathbb{P}^{1} \longrightarrow \mathbb{P}^{1}
$$

respectively. There is a contraction $\alpha: X \rightarrow \mathbb{P}^{1} \times \mathbb{P}^{1} \times \mathbb{P}^{1}$ of a surface $E \subset X$ to a curve

$$
C \subset \mathbb{P}^{1} \times \mathbb{P}^{1} \times \mathbb{P}^{1}
$$

such that $C \cdot F_{1}=C \cdot F_{2}=1$ and $C \cdot F_{3}=2$. There is a smooth surface

$$
\mathbb{P}^{1} \times \mathbb{P}^{1} \cong G \in\left|F_{1}+F_{2}\right|
$$

such that $C \subset G$. In particular, we see that

$$
-K_{X} \sim 2 \bar{G}+E+\bar{F}_{3},
$$

where $\bar{F}_{3}$ and $\bar{G}$ are proper transforms of $F_{3}$ and $G$, respectively. Hence lct $(X) \leqslant 1 / 2$.

We suppose that $\operatorname{lct}(X)<1 / 2$. Then there exists an effective $\mathbb{Q}$-divisor $D \sim_{\mathbb{Q}}-K_{X}$ such that the log pair $(X, \lambda D)$ is not log canonical for some positive rational number $\lambda<1 / 2$. Note that

$$
\varnothing \neq \operatorname{LCS}(X, \lambda D) \subseteq E
$$

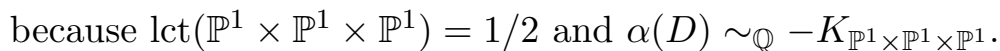

There is a smooth surface $H \in\left|3 F_{1}+F_{3}\right|$ such that $C=G \cap H$. Let $\bar{H}$ be a proper transform of the surface $H$ on the threefold $X$. Then $\bar{H} \cap \bar{G}=\varnothing$ and there is a commutative diagram

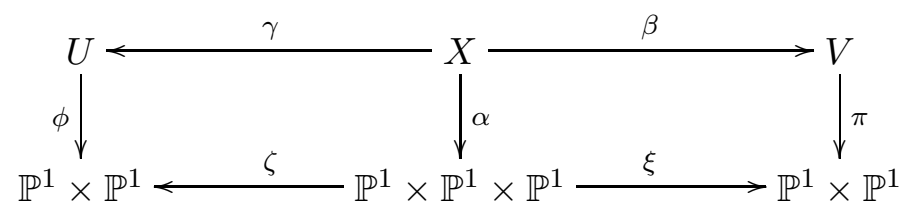

such that $\beta$ and $\gamma$ are contractions of the surfaces $\bar{G}$ and $\bar{H}$ to smooth curves, the morphisms $\pi$ and $\phi$ are $\mathbb{P}^{1}$-bundles, the morphisms $\zeta$ and $\xi$ are projections that are given by the linear systems $\left|F_{1}+F_{2}\right|$ and $\left|F_{1}+F_{3}\right|$, respectively.

It follows from $\bar{H} \cap \bar{G}=\varnothing$ that

- either the log pair $(V, \lambda \beta(D))$ is not log canonical,

- or the log pair $(U, \lambda \gamma(D))$ is not log canonical.

Applying Theorem 2.28 to the $\log$ pairs $(V, \lambda \beta(D))$ or $(U, \lambda \gamma(D))$ (and the fibrations $\pi$ or $\phi$, respectively) and using Theorem 2.7, we see that

$$
\operatorname{LCS}(X, \lambda D)=\Gamma
$$

where $\Gamma$ is a fiber of the natural projection $E \rightarrow C$.

We may assume that $\alpha(\Gamma) \in F_{3}$. Let $\bar{F}_{3} \subset X$ be the proper transform of the surface $F_{3}$. Put

$$
D=\mu \bar{F}_{3}+\Omega,
$$

where $\Omega$ is an effective $\mathbb{Q}$-divisor on $X$ such that $\bar{F}_{3} \not \subset \operatorname{Supp}(\Omega)$. Then

$$
\mu F_{3}+\alpha(\Omega) \sim_{\mathbb{Q}} 2\left(F_{1}+F_{2}+F_{3}\right)
$$

which gives $\mu \leqslant 2$. Hence the $\log$ pair $\left(\bar{F}_{3},\left.\lambda \Omega\right|_{\bar{F}_{3}}\right)$ is not $\log$ canonical along $\Gamma \subset \bar{F}_{3}$ by Theorem 2.20, But

$$
\left.\Omega\right|_{\bar{F}_{3}} \sim \mathbb{Q}-K_{\bar{F}_{3}}
$$

and $\bar{F}_{3}$ is a del Pezzo surface such that $K_{\bar{F}_{3}}^{2}=6$ and 
- either $\bar{F}_{3}$ is smooth and $\left|C \cap F_{3}\right|=2$;

- or $\bar{F}_{3}$ has one ordinary double point and $\left|C \cap F_{3}\right|=1$.

We have $\operatorname{lct}\left(\bar{F}_{3}\right) \leqslant \lambda$. Then $\bar{F}_{3}$ is singular by Example 1.18, It follows from Lemma [5.5 that

$$
\operatorname{LCS}\left(\bar{F}_{3},\left.\lambda \Omega\right|_{\bar{F}_{3}}\right)=\operatorname{Sing}\left(\bar{F}_{3}\right),
$$

but the log pair $\left(\bar{F}_{3},\left.\lambda \Omega\right|_{\bar{F}_{3}}\right)$ is not $\log$ canonical along the whole curve $\Gamma \subset \bar{F}_{3}$, which is a contradiction.

Lemma 10.4. Suppose that $I(X)=4.5$. Then $\operatorname{lct}(X)=3 / 7$.

Proof. Let $Q \subset \mathbb{P}^{4}$ be a quadric cone, let $V \subset \mathbb{P}^{6}$ be a a section of $\operatorname{Gr}(2,5) \subset \mathbb{P}^{9}$ by a linear subspace of dimension 6 such that $V$ has one ordinary double point. Then the diagram

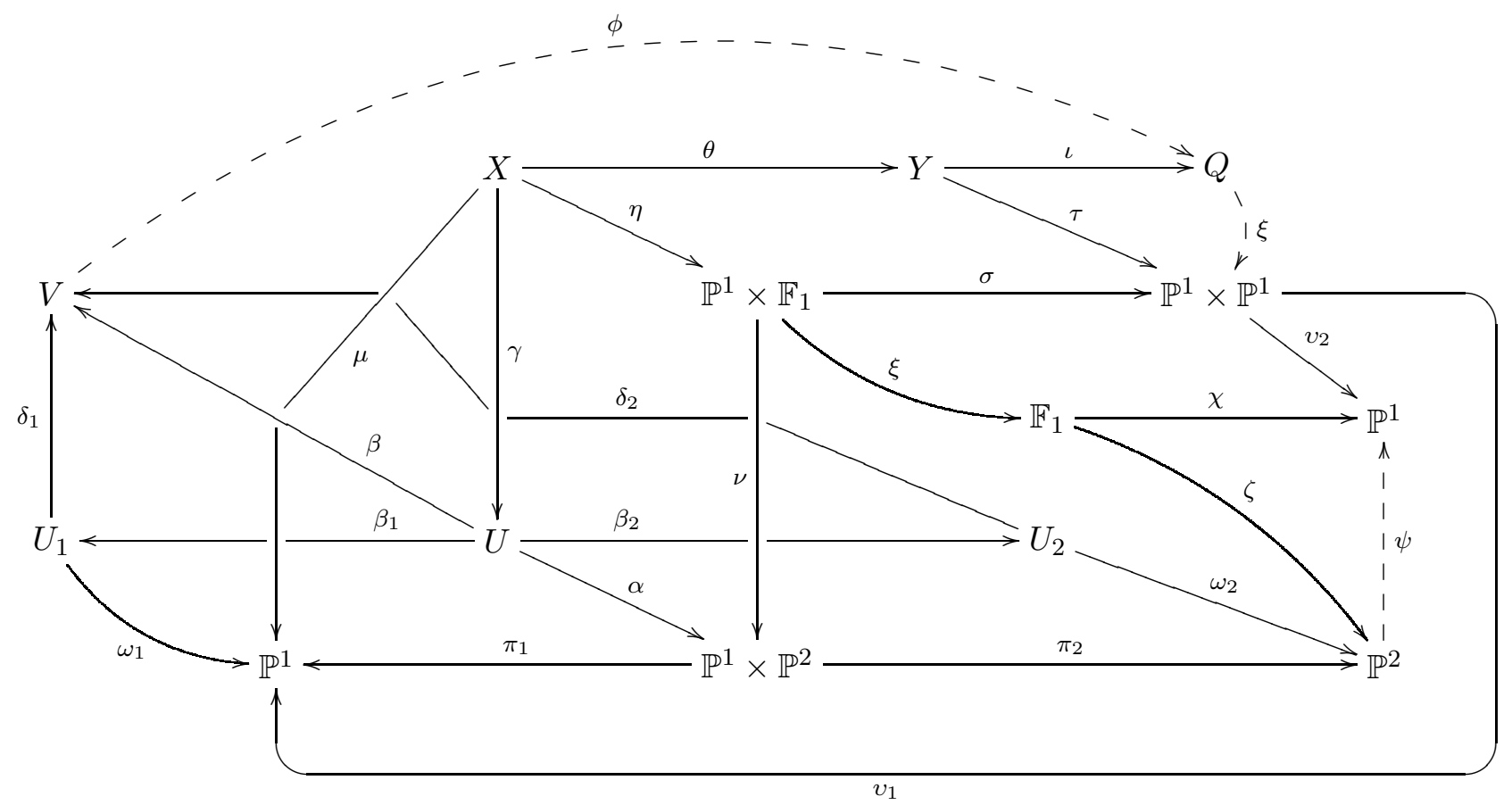

commutes (cf. 66, Lemma 2.6]), where we have the following notation:

- the morphisms $\pi_{i}, v_{i}, \xi$ and $\chi$ are natural projections;

- the morphism $\alpha$ contracts a surface $\mathbb{F}_{3} \cong E \subset U$ to a curve $C$ such that

$$
\pi_{1}^{*}\left(\mathcal{O}_{\mathbb{P}^{1}}(1)\right) \cdot C=2, \pi_{2}^{*}\left(\mathcal{O}_{\mathbb{P}^{2}}(1)\right) \cdot C=1
$$

- the morphism $\beta$ contracts a surface $\mathbb{P}^{1} \times \mathbb{P}^{1} \cong \bar{H}_{2} \subset U$ to the singular point of $V$;

- the morphism $\beta_{i}$ contracts the surface $\bar{H}_{2}$ to a smooth rational curve;

- the morphism $\delta_{i}$ contracts the curve $\beta_{i}\left(\bar{H}_{2}\right)$ to the singular point of $V$ so that the map

$$
\delta_{2} \circ \delta_{1}^{-1}: U_{1} \rightarrow U_{2}
$$

is a standard flop in the curve $\beta_{1}\left(\bar{H}_{2}\right) \cong \mathbb{P}^{1}$;

- the morphism $\omega_{1}$ is a fibration whose general fiber is $\mathbb{P}^{1} \times \mathbb{P}^{1}$;

- the morphisms $\omega_{2}, \pi_{2}, \xi, \sigma, \tau$ are $\mathbb{P}^{1}$-bundles;

- the morphism $\zeta$ is a blow up of a point $O \in \mathbb{P}^{2}$ such that $O \notin \pi_{2}(C)$;

- the map $\psi$ is a linear projection from the point $O \in \mathbb{P}^{2}$;

- the morphism $\nu$ contracts a surface $G \cong \mathbb{P}^{1} \times \mathbb{P}^{1}$ to a curve $L$ such that $\pi_{2}(L)=O$;

- the morphism $\gamma$ contracts a surface $\breve{G}$ to a curve $\bar{L}$ such that

$$
\alpha(\bar{L})=L \subset \mathbb{P}^{1} \times \mathbb{P}^{2}
$$

and the curve $\beta(\bar{L})$ is a line in $V \subset \mathbb{P}^{6}$ such that $\beta(\bar{L}) \cap \operatorname{Sing}(V)=\varnothing$;

- the morphism $\eta$ contracts a surface $\breve{E}$ to a curve such that $\nu \circ \eta(\breve{E})=C \subset \mathbb{P}^{1} \times \mathbb{P}^{2}$; 
- the morphism $\theta$ contracts a surface $\breve{R} \subset X$ to a curve such that $\breve{R} \neq \breve{E}$ and

$$
\tau \circ \theta(\breve{R})=\sigma \circ \eta(\breve{E}) \subset \mathbb{P}^{1} \times \mathbb{P}^{1} ;
$$

- the morphism $\mu$ is a fibration into del Pezzo surfaces of degree 6;

- the morphism $\iota$ contracts the surface $\theta\left(\breve{H}_{2}\right)$ to the singular point of the quadric $Q$;

- the map $\phi$ is a linear projection from the line $\beta(\bar{L}) \subset V \subset \mathbb{P}^{6}$.

The curve $\pi_{2}(C) \subset \mathbb{P}^{2}$ is a line. Then $\alpha\left(\bar{H}_{2}\right) \in\left|\pi_{2}^{*}\left(\mathcal{O}_{\mathbb{P}^{2}}(1)\right)\right|$ and $C \subset \alpha\left(\bar{H}_{2}\right)$.

The morphism $\pi_{1}$ induces a double cover $C \rightarrow \mathbb{P}^{1}$ branched in two points $Q_{1} \in C \ni Q_{2}$. Let

$$
T_{i} \in\left|\pi_{1}^{*}\left(\mathcal{O}_{\mathbb{P}^{1}}(1)\right)\right|
$$

be the unique surface such that $Q_{i} \in T_{i}$. Let $\bar{T}_{i} \subset U$ be the proper transform of $T_{i}$. Then

- the surface $\bar{T}_{i}$ has one ordinary double point,

- the surface $\bar{T}_{i}$ is tangent to the surface $E$ along the curve $E \cap \bar{T}_{i}$,

- the surface $\bar{T}_{i}$ is a del Pezzo surface such that $K_{\bar{T}_{i}}^{2}=7$.

Let $Z_{i} \subset \mathbb{P}^{2}$ be the unique line such that $O \in Z \ni \pi_{2} \circ \alpha\left(Q_{i}\right)$. Then there is a unique surface

$$
\bar{R}_{i} \in\left|\left(\pi_{2} \circ \alpha\right)^{*}\left(\mathcal{O}_{\mathbb{P}^{2}}(1)\right)\right|
$$

such that $Z_{i} \subset \pi_{2} \circ \alpha\left(\bar{R}_{i}\right)$. One has $\bar{L} \subset \bar{R}_{i}$ and

$$
-K_{U} \sim 2 \bar{H}_{2}+\bar{R}_{i}+2 \bar{T}_{i}+E .
$$

Let $\Gamma_{i}$ be a fiber of the projection $E \rightarrow C$ over the point $Q_{i}$. Then $\Gamma_{i}=E \cap \bar{T}_{i}$ and

$$
\Gamma_{i} \subset \operatorname{LCS}\left(U, \frac{3}{7}\left(2 \bar{H}_{2}+\bar{R}_{i}+2 \bar{T}_{i}+E\right)\right) .
$$

Let $\breve{R}_{i}$ and $\breve{T}_{i}$ be the proper transforms of $\bar{R}_{i}$ and $\bar{T}_{i}$ on the threefold $X$, respectively. Then

$$
-K_{X} \sim 2 \breve{H}_{2}+\breve{R}_{i}+2 \breve{T}_{i}+\breve{E},
$$

because $\bar{L} \subset \bar{R}_{i}$. Let $\breve{\Gamma}_{i} \subset X$ be the proper transform of the curve $\Gamma_{i}$. Then the log pair

$$
\left(X, \frac{3}{7}\left(2 \breve{H}_{2}+\breve{R}_{i}+2 \breve{T}_{i}+\breve{E}\right)\right)
$$

is $\log$ canonical but not $\log$ terminal. Thus, we see that $\operatorname{lct}(X) \leqslant 3 / 7$.

We suppose that $\operatorname{lct}(X)<3 / 7$. Then there exists an effective $\mathbb{Q}$-divisor $D \sim_{\mathbb{Q}}-K_{X}$ such that the log pair $(X, \lambda D)$ is not log canonical for some rational $\lambda<3 / 7$.

The surfaces $\breve{T}_{1}$ and $\breve{T}_{2}$ are the only singular fibers of the fibration $\mu: X \rightarrow \mathbb{P}^{1}$. Then

$$
\breve{T}_{i} \not \subset \operatorname{LCS}(X, \lambda D) \subsetneq \breve{T}_{1} \cup \breve{T}_{2},
$$

by Lemma 2.26, because $D \cdot Z=\breve{T}_{1}=2$, where $Z$ is a general fiber of $\pi_{2} \circ \alpha \circ \gamma$.

We may assume that $\operatorname{LCS}(X, \lambda D) \subseteq \breve{T}_{1}$ by Theorem 2.7 .

Applying Theorem 2.28 to the log pair $\left(\mathbb{P}^{1} \times \mathbb{F}_{1}, \lambda \eta(D)\right)$, we see that

$$
\varnothing \neq \operatorname{LCS}(X, \lambda D) \neq \breve{T}_{1} \cap \breve{G},
$$

because $G=\eta(\breve{G})$ is a section of the $\mathbb{P}^{1}$-bundle $\sigma$.

Applying Theorem 2.28 to the log pair $\left(\mathbb{P}^{1} \times \mathbb{P}^{2}, \lambda \alpha \circ \gamma(D)\right)$, we see that

$$
\varnothing \neq \operatorname{LCS}(X, \lambda D) \subseteq \breve{T}_{1} \cap \breve{E}=\breve{\Gamma}_{1}
$$

by Theorem 2.7, because $\breve{G} \cap \breve{E}=\varnothing$ and $T_{1}$ is a section of $\pi_{2}$.

Applying Theorem 2.28 to the log pairs $(Y, \lambda \theta(D))$ and $\left(U_{2}, \lambda \beta_{2} \circ \gamma(D)\right)$ (and the fibrations $\tau$ and $\omega_{2}$ ) we see that

$$
\varnothing \neq \operatorname{LCS}(X, \lambda D)=\breve{\Gamma}_{1},
$$

because $\breve{R} \cap \breve{H}_{2}=\varnothing$. Put $\bar{D}=\gamma(D)$. Then $\operatorname{LCS}(U, \lambda \bar{D})=\Gamma_{1}$. Put

$$
\begin{gathered}
\bar{D}=\varepsilon \bar{H}_{2}+\Omega, \\
90
\end{gathered}
$$


where $\Omega$ is an effective $\mathbb{Q}$-divisor such that $\bar{H}_{2} \not \subset \operatorname{Supp}(\Omega)$. Then

$$
\left.\Omega\right|_{\bar{H}_{2}} \sim \mathbb{Q}_{\mathbb{Q}}-\frac{(1+\varepsilon)}{2} K_{\bar{H}_{2}}
$$

and the log pair $\left(\bar{H}_{2},\left.\lambda \Omega\right|_{\bar{H}_{2}}\right)$ is not $\log$ canonical by Theorem 2.20 . The latter implies that

$$
\frac{3}{7} \cdot \frac{1+\varepsilon}{2}>\lambda \frac{1+\varepsilon}{2}>1 / 2
$$

by Lemma 2.24, and hence $\varepsilon>4 / 3$.

We may assume that either $E \nsubseteq \operatorname{Supp}(\bar{D})$ or $\bar{T}_{1} \not \subset \operatorname{Supp}(\bar{D})$ by Remark 2.23 .

Suppose that $E \nsubseteq \operatorname{Supp}(\bar{D})$. Let $Z$ be a general fiber of the projection $E \rightarrow C$. Then

$$
1=-K_{U} \cdot Z=\bar{D} \cdot Z=\varepsilon+\Omega \cdot Z \geqslant \varepsilon,
$$

which is a contradiction, because $\varepsilon>4 / 3$. Thus, we see that $\bar{T}_{1} \nsubseteq \mathbb{S u p p}(\bar{D})$.

Let $\bar{\Delta} \subset \bar{T}_{1}$ be a proper transform of a general line in $T_{1} \cong \mathbb{P}^{2}$ that passes through $Q_{1}$. Then

$$
2=-K_{U} \cdot \bar{\Delta}=\bar{D} \cdot \bar{\Delta} \geqslant \operatorname{mult}_{\Gamma_{1}}(\bar{D}) \geqslant 1 / \lambda>7 / 3,
$$

because $\bar{\Delta} \not \subset \operatorname{Supp}(\bar{D})$ and $\bar{\Delta} \cap \Gamma_{1} \neq \varnothing$. The obtained contradiction completes the proof.

Lemma 10.5. Suppose that $I(X)=4.6$. Then $\operatorname{lct}(X)=1 / 2$.

Proof. There is a birational morphism $\alpha: X \rightarrow \mathbb{P}^{3}$ that blows up three disjoint lines $L_{1}, L_{2}, L_{3}$.

Let $H_{i}$ be the proper transform on $X$ of a general plane in $\mathbb{P}^{3}$ such that $L_{i} \subset \alpha\left(H_{i}\right)$. Then

$$
-K_{X} \sim 2 H_{1}+E_{1}+H_{2}+H_{3} \sim 2 H_{2}+E_{2}+H_{1}+H_{3} \sim 2 H_{3}+E_{3}+H_{1}+H_{2},
$$

where $E_{i}$ is the exceptional divisor of $\alpha$ such that $\alpha\left(E_{i}\right)=L_{i}$. In particular, we see that $\operatorname{lct}(X) \leqslant 1 / 2$.

We suppose that $\operatorname{lct}(X)<1 / 2$. Then there exists an effective $\mathbb{Q}$-divisor $D \sim_{\mathbb{Q}}-K_{X}$ such that the log pair $(X, \lambda D)$ is not log canonical for some positive rational number $\lambda<1 / 2$.

The surface $H_{i}$ is a smooth del Pezzo surface such that $K_{H_{i}}^{2}=7$, the linear system $\left|H_{i}\right|$ has no base points and induces a smooth morphism $\phi_{i}: X \rightarrow \mathbb{P}^{1}$, whose fibers are isomorphic to $H_{i}$.

Suppose that $|\operatorname{LCS}(X, \lambda D)|<+\infty$. We may assume that $\operatorname{LCS}(X, \lambda D) \nsubseteq E_{1}$. Then the set

$$
\operatorname{LCS}\left(X, \lambda D+H_{1}+\frac{1}{2} E_{1}\right)
$$

is disconnected, which is impossible by Theorem 2.7, because $H_{2}+H_{3}+(\lambda-1 / 2) K_{X}$ is ample.

We may assume that $H_{1} \cap \operatorname{LCS}(X, \lambda D) \neq \varnothing$. Then

$$
\varnothing \neq H_{1} \cap \operatorname{LCS}(X, \lambda D) \subseteq \operatorname{LCS}\left(H_{1},\left.\lambda D\right|_{H_{1}}\right)
$$

by Remark 2.3. Put $C_{2}=\left.E_{2}\right|_{H_{1}}$ and $C_{3}=\left.E_{3}\right|_{H_{1}}$. Then

$$
C_{2} \cdot C_{2}=C_{3} \cdot C_{3}=-1
$$

and there is a unique curve $\mathbb{P}^{1} \cong C \subset H_{1}$ such that $C \cdot C_{2}=C \cdot C_{3}=1$ and $C \cdot C=-1$. Note that

by Lemma 5.9 .

$$
\operatorname{LCS}\left(H_{1},\left.\lambda D\right|_{H_{1}}\right)=C
$$

There is a unique smooth quadric $Q \subset \mathbb{P}^{3}$ that contains $L_{1}, L_{2}, L_{3}$. Note that

$$
\bar{Q} \cap H_{1}=C,
$$

where $\bar{Q} \subset X$ is a proper transform of the surface $Q$.

There is a morphism $\sigma: X \rightarrow \mathbb{P}^{1} \times \mathbb{P}^{1} \times \mathbb{P}^{1}$ contracting $\bar{Q}$ to a curve of tri-degree $(1,1,1)$. Since $\bar{Q} \cap H_{1}=C$, one obtains (see Remark 2.3) that

$$
\operatorname{LCS}(X, \lambda D) \supset \bar{Q}
$$

and hence $\operatorname{LCS}(X, \lambda D)=\bar{Q}$, because $\operatorname{lct}\left(\mathbb{P}^{1} \times \mathbb{P}^{1} \times \mathbb{P}^{1}\right)=1 / 2$. Put

$$
\begin{gathered}
D=\mu \bar{Q}+\Omega, \\
91
\end{gathered}
$$


where $\mu \geqslant 1 / \lambda>2$, and $\Omega$ is an effective $\mathbb{Q}$-divisor such that $\bar{Q} \not \subset \operatorname{Supp}(\Omega)$. Then

$$
\alpha(D)=\mu Q+\alpha(\Omega),
$$

which is impossible, because $\alpha(D) \sim_{\mathbb{Q}} 2 Q \sim-K_{\mathbb{P} 3}$ and $\mu>2$.

Lemma 10.6. Suppose that $I(X)=4$.7. Then $\operatorname{lct}(X)=1 / 2$.

Proof. There is a birational morphism $\alpha: X \rightarrow W$ such that

- the variety $W$ is a smooth divisor of bi-degree $(1,1)$ on $\mathbb{P}^{2} \times \mathbb{P}^{2}$;

- the morphism $\alpha$ contracts two (irreducible) surfaces $E_{1} \neq E_{2}$ to two disjoint curves $L_{1}$ and $L_{2}$;

- the curves $L_{i}$ are fibers of one natural $\mathbb{P}^{1}$-bundle $W \rightarrow \mathbb{P}^{2}$.

There is a surface $H \subset W$ such that $-K_{X} \sim 2 H$ and $L_{1} \subset H \supset L_{2}$. Then

$$
-K_{X} \sim 2 \bar{H}+E_{1}+E_{2},
$$

where $\bar{H}$ is a proper transform of $H$ on the threefold $X$. In particular, $\operatorname{lct}(X) \leqslant 1 / 2$.

We suppose that $\operatorname{lct}(X)<1 / 2$. Then there exists an effective $\mathbb{Q}$-divisor $D \sim_{\mathbb{Q}}-K_{X}$ such that the log pair $(X, \lambda D)$ is not log canonical for some $\lambda<1 / 2$. Then

$$
\varnothing \neq \operatorname{LCS}(X, \lambda D) \subseteq E_{1} \cup E_{2},
$$

since $\operatorname{lct}(W)=1 / 2$ by Theorem 7.1 and $\alpha(D) \sim \mathbb{Q}-K_{W}$.

We may assume that $\operatorname{LCS}(X, \lambda D) \cap E_{1} \neq \varnothing$. Let $\beta: X \rightarrow Y$ be a contraction of $E_{2}$. Then

$$
\mathbb{L} \mathbb{C S}(Y, \lambda \beta(D)) \neq \varnothing
$$

and $\beta(D) \sim_{\mathbb{Q}}-K_{Y}$, which contradicts Lemma 9.25,

Lemma 10.7. Suppose that $I(X)=4$. . Then $\operatorname{lct}(X)=1 / 3$.

Proof. There is blow up $\alpha: X \rightarrow \mathbb{P}^{1} \times \mathbb{P}^{1} \times \mathbb{P}^{1}$ of a curve $C \subset \mathbb{P}^{1} \times \mathbb{P}^{1} \times \mathbb{P}^{1}$ such that $C \subset F_{1}$ and

$$
C \cdot F_{2}=C \cdot F_{3}=1 \text {, }
$$

where $F_{i}$ is a fiber of the projection of $\mathbb{P}^{1} \times \mathbb{P}^{1} \times \mathbb{P}^{1}$ to its $i$-th factor. There is a surface

$$
\mathbb{P}^{1} \times \mathbb{P}^{1} \cong G \in\left|F_{2}+F_{3}\right|
$$

such that $C \subset G$. Let $E$ be the exceptional divisor of $\alpha$. Then

$$
-K_{X} \sim 2 \bar{F}_{1}+2 \bar{G}+3 E,
$$

where $\bar{F}_{1}$ and $\bar{G}$ are proper transforms of $F_{1}$ and $G$, respectively. In particular, $\operatorname{lct}(X) \leqslant 1 / 3$.

We suppose that $\operatorname{lct}(X)<1 / 3$. Then there exists an effective $\mathbb{Q}$-divisor $D \sim_{\mathbb{Q}}-K_{X}$ such that the log pair $(X, \lambda D)$ is not log canonical for some positive rational number $\lambda<1 / 3$. Note that

$$
\varnothing \neq \operatorname{LCS}(X, \lambda D) \subseteq E,
$$

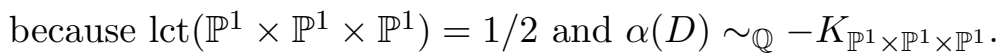

Let $Q$ be a quadric cone in $\mathbb{P}^{4}$. Then there is a commutative diagram

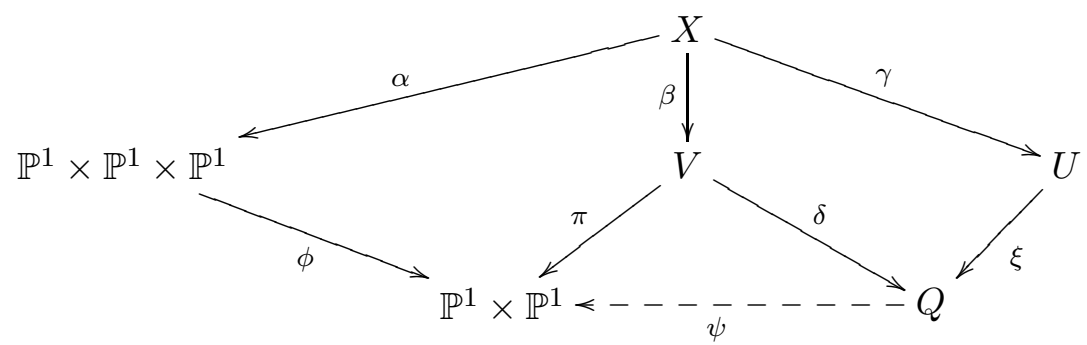

where we have the following notations:

- $V$ is a variety with $I(V)=3.31$;

- the morphism $\beta$ is a contraction of the surface $\bar{G}$ to a curve;

- the morphism $\gamma$ is a contraction of $\bar{F}_{1} \cong \mathbb{P}^{1} \times \mathbb{P}^{1}$ to an ordinary double point; 
- the morphism $\delta$ is a blow up of the vertex of the quadric cone $Q \subset \mathbb{P}^{4}$;

- the morphism $\xi$ is a blow up of a smooth conic in $Q$;

- the map $\psi$ is a projection from the vertex of the cone $Q$;

- the morphism $\phi$ is a projection that is given by $\left|F_{2}+F_{3}\right|$, i. e. the projection of $\mathbb{P}^{1} \times \mathbb{P}^{1} \times \mathbb{P}^{1}$ onto the product of the last two factors;

- the morphism $\pi$ is a natural $\mathbb{P}^{1}$-bundle.

It follows from Corollary 6.4 that $\operatorname{lct}(V)=1 / 3$. On the other hand, $\operatorname{lct}(U)=1 / 3$ by Lemma 2.27, Hence

$$
\varnothing \neq \operatorname{LCS}(X, \lambda D) \subseteq E \cap \bar{G} \cap \bar{F}_{1}=\varnothing,
$$

which is a contradiction.

Lemma 10.8. Suppose that $I(X)=4.9$. Then $\operatorname{lct}(X)=1 / 3$.

Proof. There exists a point $O \in \mathbb{P}^{3}$, and there exist lines $L_{1} \subset \mathbb{P}^{3} \supset L_{2}$ such that $L_{1} \cap L_{2}=\varnothing$, the line $L_{1}$ passes through the point $O$, and there is a commutative diagram

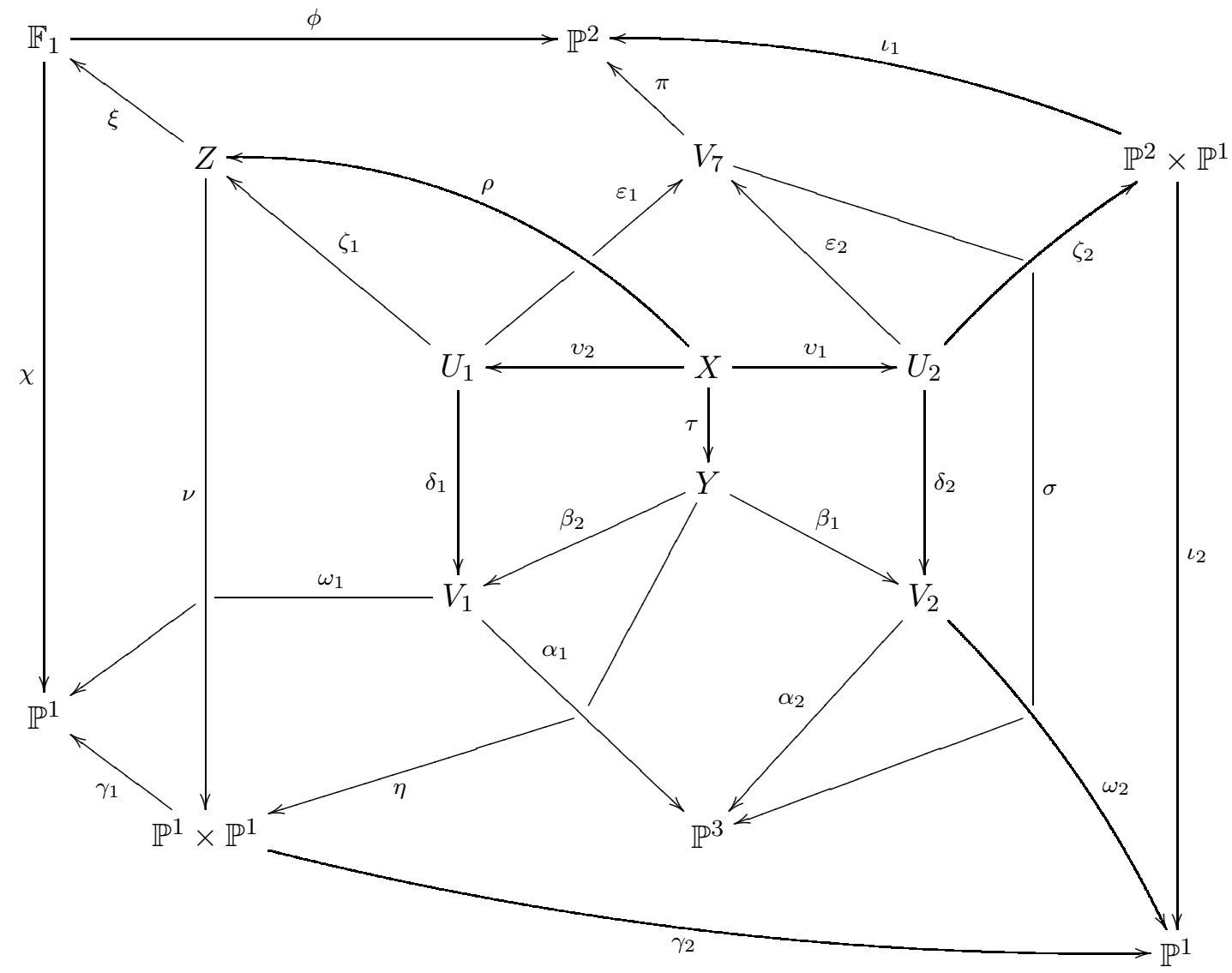

that uses the following notation:

- the morphism $\sigma$ is a blow up of the point $O$;

- the morphism $\pi$ is a natural $\mathbb{P}^{1}$-bundle;

- the morphism $\alpha_{i}$ is a blow up of the line $L_{i}$;

- the morphisms $\beta_{i}, v_{i}$ and $\varepsilon_{i}$ are blow ups of the proper transforms of the line $L_{i}$;

- the morphisms $\tau$ and $\delta_{1}$ are blow ups of curves that are the preimages of the point $O$;

- the morphism $\delta_{2}$ is a blow up of the point that dominates the point $O$;

- the morphisms $\omega_{1}$ and $\omega_{2}$ are natural $\mathbb{P}^{2}$-bundles, where

$$
V_{1} \cong V_{2} \cong \mathbb{P}\left(\mathcal{O}_{\mathbb{P}^{1}} \oplus \mathcal{O}_{\mathbb{P}^{1}} \oplus \mathcal{O}_{\mathbb{P}^{1}}(1)\right)
$$

- the morphisms $\iota_{1}, \iota_{2}, \gamma_{1}$ and $\gamma_{2}$ are natural projections;

- the morphism $\phi$ is a blow up of the point $\pi\left(\bar{L}_{1}\right)$, where $\bar{L}_{1} \subset V_{7}$ is a proper transform of $L_{1}$ 
- the morphisms $\rho$ and $\zeta_{i}$ contract the proper transforms of the plane $\Pi \subset \mathbb{P}^{3}$ such that

$$
L_{2} \subset \Pi \ni O ;
$$

- the morphisms $\eta, \nu, \xi, \chi, \gamma_{1}, \gamma_{2}$ and $\iota_{2}$ are natural $\mathbb{P}^{1}$-bundles.

Let $H_{i} \subset X$ be the proper transform of a general plane in $\mathbb{P}^{3}$ that contains $L_{i}$. Then

$$
-K_{X} \sim 3 H_{1}+H_{2}+2 E_{1}+G,
$$

where $E_{i}$ and $G$ be the exceptional divisor of $v_{i}$ and $\tau$, respectively. Thus, we have $\operatorname{lct}(X) \leqslant 1 / 3$.

We suppose that $\operatorname{lct}(X)<1 / 3$. Then there exists an effective $\mathbb{Q}$-divisor $D \sim_{\mathbb{Q}}-K_{X}$ such that the log pair $(X, \lambda D)$ is not log canonical for some positive rational number $\lambda<1 / 3$. Note that

$$
\varnothing \neq \operatorname{LCS}(X, \lambda D) \subseteq G,
$$

since $\operatorname{lct}(Y)=1 / 3$ by Lemma 9.26. But the surface $G$ is not a fiber of the smooth morphism

$$
\omega_{1} \circ \beta_{2} \circ \tau: X \longrightarrow \mathbb{P}^{1},
$$

so we obtain a contradiction applying Lemma 2.26 to the morphism $\omega_{1} \circ \beta_{2} \circ \tau$.

The proof of Lemma 10.8 implies the following.

Corollary 10.9. Suppose that $I(X)=5.2$. Then $\operatorname{lct}(X)=1 / 3$.

Remark 10.10. Actually, the results of Lemma 10.8 and Corollary 10.9 are contained in Corollary 6.4, but we still prefer to give a detailed proof that may have further applications.

The following result is implied by Corollaries 9.19 and 10.9, Lemma 2.30 and Example 1.18 .

Corollary 10.11. Suppose that $\rho \geqslant 5$. Then

$$
\operatorname{lct}(X)=\left\{\begin{array}{l}
1 / 3 \text { whenever } I(X) \in\{5.1,5.2\}, \\
1 / 2 \text { in the remaining cases. }
\end{array}\right.
$$

Lemma 10.12. Suppose that $I(X)=4.13$ and $X$ is general. Then $\operatorname{lct}(X)=1 / 2$.

Proof. Let $F_{1} \cong F_{2} \cong F_{3} \cong \mathbb{P}^{1} \times \mathbb{P}^{1}$ be fibers of three different projections

$$
\mathbb{P}^{1} \times \mathbb{P}^{1} \times \mathbb{P}^{1} \longrightarrow \mathbb{P}^{1}
$$

respectively. There is a contraction $\alpha: X \rightarrow \mathbb{P}^{1} \times \mathbb{P}^{1} \times \mathbb{P}^{1}$ of a surface $E \subset X$ to a curve

$$
C \subset \mathbb{P}^{1} \times \mathbb{P}^{1} \times \mathbb{P}^{1}
$$

such that $C \cdot F_{1}=C \cdot F_{2}=1$ and $C \cdot F_{3}=3$. Then there is a smooth surface

$$
\mathbb{P}^{1} \times \mathbb{P}^{1} \cong G \in\left|F_{1}+F_{2}\right|
$$

such that $C \subset G$. In particular, we see that

$$
-K_{X} \sim 2 \bar{G}+E+2 \bar{F}_{3},
$$

where $\bar{F}_{3}$ and $\bar{G}$ are proper transforms of $F_{3}$ and $G$, respectively. Hence $\operatorname{lct}(X) \leqslant 1 / 2$.

We suppose that $\operatorname{lct}(X)<1 / 2$. Then there exists an effective $\mathbb{Q}$-divisor $D \sim_{\mathbb{Q}}-K_{X}$ such that the log pair $(X, \lambda D)$ is not $\log$ canonical for some positive rational number $\lambda<1 / 2$. Note that

$$
\varnothing \neq \operatorname{LCS}(X, \lambda D) \subseteq E \cong \mathbb{F}_{4},
$$

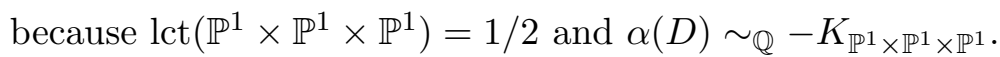

There are smooth surfaces $H_{1} \in\left|3 F_{1}+F_{3}\right|$ and $H_{2} \in\left|3 F_{2}+F_{3}\right|$ such that

$$
C=G \cdot H_{1}=G \cdot H_{2},
$$

and $H_{1} \cong H_{2} \cong \mathbb{P}^{1} \times \mathbb{P}^{1}$. Let $\bar{H}_{i}$ be a proper transform of $H_{i}$ on the threefold $X$. Then

$$
\bar{H}_{1} \cap \bar{G}=\underset{94}{\bar{H}_{2} \cap \bar{G}=\varnothing .}
$$


There is a commutative diagram

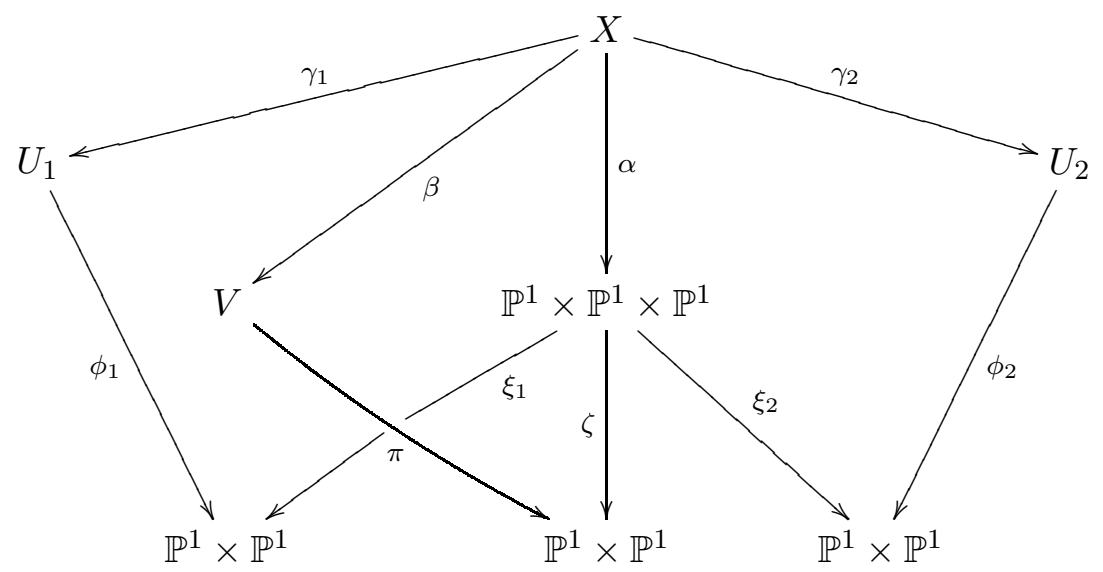

such that $\beta$ and $\gamma_{i}$ are contractions of the surfaces $\bar{G}$ and $\bar{H}_{i}$ to a smooth curves, the morphisms $\pi$ and $\phi_{i}$ are $\mathbb{P}^{1}$-bundles, the morphisms $\zeta$ and $\xi_{i}$ are projections that are given by the linear systems $\left|F_{1}+F_{2}\right|$ and $\left|F_{i}+F_{3}\right|$, respectively.

It follows from $\bar{H}_{1} \cap \bar{G}=\varnothing$ that

- either the log pair $(V, \lambda \beta(D))$ is not log canonical,

- of the log pair $\left(U_{1}, \lambda \gamma_{1}(D)\right)$ is not $\log$ canonical.

Applying Theorem 2.28 to $(V, \lambda \beta(D))$ or $\left(U_{1}, \lambda \gamma_{1}(D)\right)$ (and the fibration $\pi$ or $\phi_{1}$ ) and using Theorem 2.7, we see that

$$
\operatorname{LCS}(X, \lambda D)=\Gamma
$$

where $\Gamma$ is a fiber of the natural projection $E \rightarrow C$.

We may assume that $\alpha(\Gamma) \in F_{3}$. Let $\bar{F}_{3} \subset X$ be the proper transform of the surface $F_{3}$. Put

$$
D=\mu \bar{F}_{3}+\Omega,
$$

where $\Omega$ is an effective $\mathbb{Q}$-divisor on $X$ such that $\bar{F}_{3} \not \subset \operatorname{Supp}(\Omega)$. Then

$$
\mu F_{3}+\alpha(\Omega) \sim_{\mathbb{Q}} 2\left(F_{1}+F_{2}+F_{3}\right),
$$

which gives $\mu \leqslant 2$. The log pair $\left(\bar{F}_{3},\left.\lambda \Omega\right|_{\bar{F}_{3}}\right)$ is not $\log$ canonical along $\Gamma \subset \bar{F}_{3}$ by Theorem 2.20 . One has

$$
\left.\Omega\right|_{\bar{F}_{3}} \sim \mathbb{Q}-K_{\bar{F}_{3}}
$$

and $\bar{F}_{3}$ is a del Pezzo surface such that $K_{\bar{F}_{3}}^{2}=5$. Note that $\bar{F}_{3}$ may be singular. Namely, we have

$$
\operatorname{Sing}\left(\bar{F}_{3}\right)=\varnothing \Longleftrightarrow\left|C \cap F_{3}\right|=F_{3} \cdot C=3,
$$

and $\operatorname{Sing}\left(\bar{F}_{3}\right) \subset \Gamma$. The following cases are possible:

- the surface $\bar{F}_{3}$ is smooth and $\left|C \cap F_{3}\right|=3$;

- the surface $\bar{F}_{3}$ has one ordinary double point and $\left|C \cap F_{3}\right|=2$;

- the surface $\bar{F}_{3}$ has a singular point of type $\mathbb{A}_{2}$ and $\left|C \cap F_{3}\right|=1$.

We have $\operatorname{lct}\left(\bar{F}_{3}\right) \leqslant \lambda<1 / 2$. Thus, it follows from Examples 1.18 and 5.3 that $\left|C \cap F_{3}\right|=1$, which is impossible if the threefold $X$ is sufficiently general.

\section{UPPER BOUNDS}

We use the assumptions and notation introduced in section 1. The purpose of this section is to find upper bounds for the global log canonical thresholds of the varieties $X$ with

$$
J(X) \in\{1.1,1.2, \ldots, 1.17,2.1, \ldots, 2.36,3.1, \ldots, 3.31,4.1, \ldots, 4.13,5.1, \ldots, 5.7,5.8\} .
$$

Lemma 11.1. Suppose that $I(X)=1.8$. Then $\operatorname{lct}(X) \leqslant 6 / 7$. 
Proof. The linear system $\left|-K_{X}\right|$ does not have base points and induces an embedding $X \subset \mathbb{P}^{10}$, and the threefold $X$ contains a line $L \subset X$ (see [168], [178]).

It follows from [98, Theorem 4.3.3] (see [45], [178]) that there is a commutative diagram

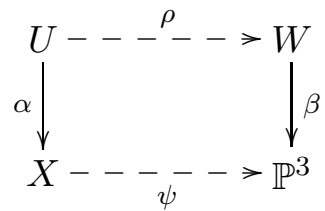

where $\alpha$ is a blow up of the line $L$, the map $\rho$ is a composition of flops, the morphism $\beta$ is a blow up of a smooth curve of degree 7 and genus 3 , and $\psi$ is a double projection from $L$.

Let $S \subset X$ be the proper transform of the exceptional surface of $\beta$. Then

$$
\operatorname{mult}_{L}(S)=7
$$

and $S \sim-3 K_{X}$, which implies that $\operatorname{lct}(X) \leqslant 6 / 7$.

Lemma 11.2. Suppose that $I(X)=1.9$. Then $\operatorname{lct}(X) \leqslant 4 / 5$.

Proof. The linear system $\left|-K_{X}\right|$ does not have base points and induces an embedding $X \subset \mathbb{P}^{11}$, and the threefold $X$ contains a line $L \subset X$ (see [168], [178]).

It follows from [98, Theorem 4.3.3] (see [45], [178]) that there is a commutative diagram

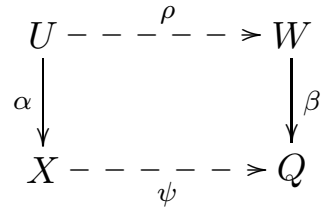

where $Q \subset \mathbb{P}^{4}$ is a smooth quadric threefold, $\alpha$ is a blow up of the line $L$, the map $\rho$ is a composition of flops, the morphism $\beta$ is a blow up along a smooth curve of degree 7 and genus 2 , and $\psi$ is a double projection from the line $L$.

Let $S \subset X$ be the proper transform of the exceptional surface of $\beta$. Then

$$
\operatorname{mult}_{L}(S)=5
$$

and $S \sim-2 K_{X}$, which implies that $\operatorname{lct}(X) \leqslant 4 / 5$.

Lemma 11.3. Suppose that $I(X)=1.10$. Then $\operatorname{lct}(X) \leqslant 2 / 3$.

Proof. The linear system $\left|-K_{X}\right|$ does not have base points and induces an embedding $X \subset \mathbb{P}^{13}$, and the threefold $X$ contains a line $L \subset X$ (see [168, [178]).

It follows from [98, Theorem 4.3.3] (see [45], [178]) that the diagram

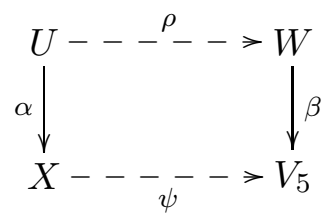

commutes, where $V_{5}$ is a smooth section of $\operatorname{Gr}(2,5) \subset \mathbb{P}^{9}$ by a linear subspace of dimension 6 , the morphism $\alpha$ is a blow up of the line $L$, the map $\rho$ is a composition of flops, the morphism $\beta$ is a blow up of a smooth rational curve of degree 5 , and $\psi$ is a double projection from $L$.

Let $S \subset X$ be the proper transform of the exceptional surface of $\beta$. Then

$$
\operatorname{mult}_{L}(S)=3
$$

and $S \sim-K_{X}$, which implies that $\operatorname{lct}(X) \leqslant 2 / 3$.

Lemma 11.4. Suppose that $I(X)=2.2$. Then $\operatorname{lct}(X) \leqslant 13 / 14$. 
Proof. There is a smooth divisor $B \subset \mathbb{P}^{1} \times \mathbb{P}^{2}$ of bi-degree $(2,4)$ such that the diagram

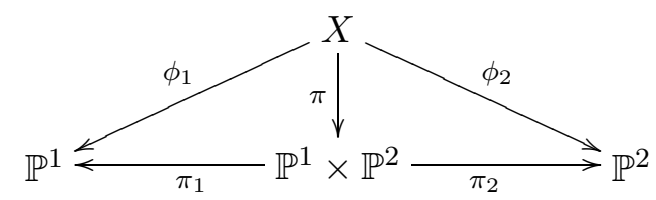

commutes, where $\pi$ is a double cover branched along $B$, the morphisms $\pi_{1}$ and $\pi_{2}$ are natural projections, $\phi_{1}$ is a fibration into del Pezzo surfaces of degree 2, and $\phi_{2}$ is a conic bundle.

Let $H_{1}$ be a general fiber of $\phi_{1}$. Put $\bar{H}_{1}=\pi\left(H_{1}\right)$. Then the intersection

$$
C=\bar{H}_{1} \cap B \subset \bar{H}_{1} \cong \mathbb{P}^{2}
$$

is a smooth quartic curve.

There is a point $P \in C$ such that

$$
\operatorname{mult}_{P}(C \cdot L) \geqslant 3
$$

where $L \subset \bar{H}_{1} \cong \mathbb{P}^{2}$ is a line that is tangent to $C$ at the point $P$.

The curve $\pi_{2}(L)$ is a line. Thus, there is a unique surface

$$
H_{2} \in\left|\phi_{2}^{*}\left(\mathcal{O}_{\mathbb{P}^{2}}(1)\right)\right|
$$

such that $\phi_{2}\left(H_{2}\right)=\pi_{2}(L)$. Hence $-K_{X} \sim H_{1}+H_{2}$.

Let us show that $\operatorname{lct}\left(X, H_{1}+H_{2}\right) \leqslant 13 / 14$. Put $\bar{H}_{2}=\pi\left(H_{2}\right)$. Then

$$
\mathbb{L} \mathbb{C S}\left(X, \frac{13}{14}\left(H_{1}+H_{2}\right)\right) \neq \varnothing \Longleftrightarrow \mathbb{L} \mathbb{C S}\left(\mathbb{P}^{1} \times \mathbb{P}^{2}, \frac{1}{2} B+\frac{13}{14}\left(\bar{H}_{1}+\bar{H}_{2}\right)\right) \neq \varnothing
$$

by [105, Proposition 3.16]. Let $\alpha: V \rightarrow \mathbb{P}^{1} \times \mathbb{P}^{2}$ be a blow up of the curve $C$. Then

$$
K_{V}+\frac{1}{2} \tilde{B}+\frac{13}{14}\left(\tilde{H}_{1}+\tilde{H}_{2}\right)+\frac{3}{7} E \sim_{\mathbb{Q}} \alpha^{*}\left(K_{\mathbb{P}^{1} \times \mathbb{P}^{2}}+\frac{1}{2} B+\frac{13}{14}\left(\bar{H}_{1}+\bar{H}_{2}\right)\right),
$$

where $\tilde{B}, \tilde{H}_{1}, \tilde{H}_{2} \subset V$ are proper transforms of $B, \bar{H}_{1}, \bar{H}_{2}$, respectively. But the log pair

$$
\left(V, \frac{13}{14} \tilde{H}_{2}+\frac{3}{7} E\right)
$$

is not $\log$ terminal along the fiber $\Gamma$ of the morphism $\alpha$ such that $\alpha(\Gamma)=P$, because

$$
\operatorname{mult}_{\Gamma}\left(\tilde{H}_{2} \cdot E\right)=\operatorname{mult}_{P}\left(C \cdot \bar{H}_{2}\right) \geqslant \operatorname{mult}_{P}(C \cdot L) \geqslant 3
$$

due to the choice of the fiber $H_{1}$. We see that

$$
\Gamma \subseteq \operatorname{LCS}\left(V, \frac{13}{14} \tilde{H}_{2}+\frac{3}{7} E\right) \subseteq \operatorname{LCS}\left(V, \frac{1}{2} \tilde{B}+\frac{13}{14}\left(\tilde{H}_{1}+\tilde{H}_{2}\right)+\frac{3}{7} E\right),
$$

which implies that $\operatorname{lct}\left(X, H_{1}+H_{2}\right) \leqslant 13 / 14$. Hence the inequality $\operatorname{lct}(X) \leqslant 13 / 14$ holds.

Remark 11.5. It follows from Lemmas 2.26 and 5.1 that $\operatorname{lct}(X) \geqslant 2 / 3$ if $I(X)=2.2$ and the threefold $X$ satisfies the following generality condition: any fiber of $\phi_{1}$ satisfies the hypotheses of Lemma 5.1

Lemma 11.6. Suppose that $I(X)=2.7$. Then $\operatorname{lct}(X) \leqslant 2 / 3$.

Proof. There is a commutative diagram

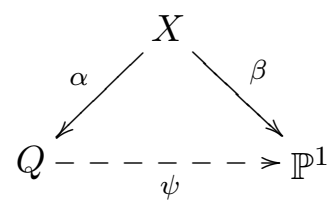

where $Q \subset \mathbb{P}^{4}$ is a smooth quadric threefold, $\alpha$ is a blow up of a smooth curve that is a complete intersection of two divisors

$$
S_{1}, S_{2} \in \underset{97}{\left|\mathcal{O}_{\mathbb{P}^{4}}(2)\right|_{Q} \mid,}
$$


the morphism $\beta$ is a fibration into del Pezzo surfaces of degree 4 , and $\psi$ is a rational map that is induced by the pencil generated by the surfaces $S_{1}$ and $S_{2}$. Then $\operatorname{lct}(X) \leqslant 2 / 3$, because

$$
-K_{X} \sim \mathbb{Q} \frac{3}{2} \bar{S}_{1}+\frac{1}{2} E
$$

where $\bar{S}_{1} \subset X$ is a proper transform of the surface $S_{1}$, and $E$ is the exceptional divisor of $\alpha$.

Lemma 11.7. Suppose that $I(X)=2.9$. Then $\operatorname{lct}(X) \leqslant 3 / 4$.

Proof. There is a commutative diagram

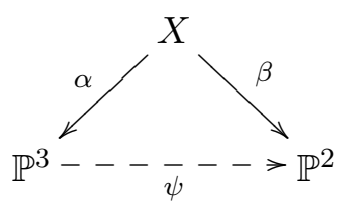

where $\alpha$ is a blow up of a smooth curve $C \subset \mathbb{P}^{3}$ of degree 7 and genus 5 that is a scheme-theoretic intersection of cubic surfaces in $\mathbb{P}^{3}$, the morphism $\beta$ is a conic bundle, and $\psi$ is a rational map that is given by the linear system of cubic surfaces that contain $C$. One has

$$
-K_{X} \sim_{\mathbb{Q}} \frac{4}{3} S+\frac{1}{3} E,
$$

where $S \in\left|\beta^{*}\left(\mathcal{O}_{\mathbb{P}^{2}}(1)\right)\right|$, and $E$ is the exceptional divisor of $\alpha$. We see that $\operatorname{lct}(X) \leqslant 3 / 4$.

Lemma 11.8. Suppose that $I(X)=2.12$. Then $\operatorname{lct}(X) \leqslant 3 / 4$.

Proof. There is a commutative diagram

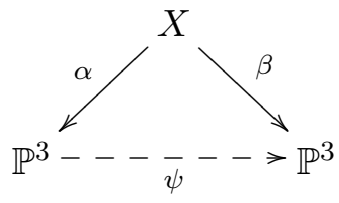

where $\alpha$ and $\beta$ are blow ups of smooth curves $C \subset \mathbb{P}^{3}$ and $Z \subset \mathbb{P}^{3}$ of degree 6 and genus 3 that are scheme-theoretic intersections of cubic surfaces in $\mathbb{P}^{3}$, and $\psi$ is a birational map that is given by the linear system of cubic surfaces that contain $C$. Then

$$
-K_{X} \sim_{\mathbb{Q}} \frac{4}{3} S+\frac{1}{3} E,
$$

where $S \in\left|\beta^{*}\left(\mathcal{O}_{\mathbb{P}^{3}}(1)\right)\right|$, and $E$ is the exceptional divisor of $\alpha$. We see that $\operatorname{lct}(X) \leqslant 3 / 4$.

Lemma 11.9. Suppose that $I(X)=2.13$. Then $\operatorname{lct}(X) \leqslant 2 / 3$.

Proof. There is a commutative diagram

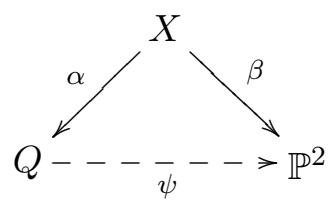

where $Q \subset \mathbb{P}^{4}$ is a smooth quadric threefold, $\alpha$ is a blow up of a smooth curve $C \subset Q$ of degree 6 and genus 2, the morphism $\beta$ is a conic bundle, and $\psi$ is a rational map that is given by the linear system of surfaces in $\left|\mathcal{O}_{\mathbb{P}^{4}}(2)\right|_{Q} \mid$ that contain the curve $C$. One has

$$
-K_{X} \sim_{\mathbb{Q}} \frac{3}{2} S+\frac{1}{2} E
$$

where $S \in\left|\beta^{*}\left(\mathcal{O}_{\mathbb{P}^{2}}(1)\right)\right|$, and $E$ is the exceptional divisor of $\alpha$. We see that $\operatorname{lct}(X) \leqslant 2 / 3$.

Lemma 11.10. Suppose that $I(X)=2.16$. Then $\operatorname{lct}(X) \leqslant 1 / 2$. 
Proof. There is a commutative diagram

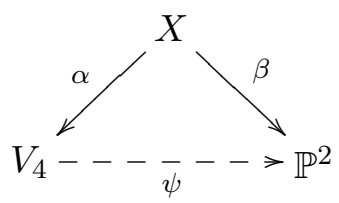

where $V_{4} \subset \mathbb{P}^{5}$ is a smooth complete intersection of two quadric hypersurfaces, $\alpha$ is a blow up of an irreducible conic $C \subset V_{4}$, the morphism $\beta$ is a conic bundle, and $\psi$ is a rational map that is given by the linear system of surfaces in $\left|\mathcal{O}_{\mathbb{P}^{5}}(1)\right|_{V_{4}} \mid$ that contain $C$. One has

$$
-K_{X} \sim 2 S+E
$$

where $S \in\left|\beta^{*}\left(\mathcal{O}_{\mathbb{P}^{2}}(1)\right)\right|$, and $E$ is the exceptional divisor of $\alpha$. We see that $\operatorname{lct}(X) \leqslant 1 / 2$.

Lemma 11.11. Suppose that $I(X)=2.17$. Then $\operatorname{lct}(X) \leqslant 2 / 3$.

Proof. There is a commutative diagram

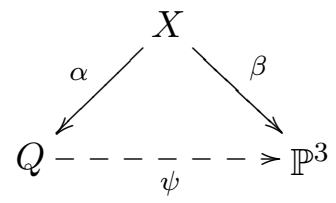

where $Q \subset \mathbb{P}^{4}$ is a smooth quadric threefold, the morphisms $\alpha$ and $\beta$ are blow ups of smooth elliptic curves $C \subset Q$ and $Z \subset \mathbb{P}^{3}$ of degree 5 , respectively, and the map $\psi$ is given by the linear system of surfaces in $\left|\mathcal{O}_{\mathbb{P}^{4}}(2)\right|_{Q} \mid$ that contain the curve $C$. One has

$$
-K_{X} \sim_{\mathbb{Q}} \frac{3}{2} S+\frac{1}{2} E,
$$

where $S \in\left|\beta^{*}\left(\mathcal{O}_{\mathbb{P}^{3}}(1)\right)\right|$, and $E$ is the exceptional divisor of $\alpha$. We see that $\operatorname{lct}(X) \leqslant 2 / 3$.

Lemma 11.12. Suppose that $I(X)=2.20$. Then $\operatorname{lct}(X) \leqslant 1 / 2$.

Proof. There is a commutative diagram

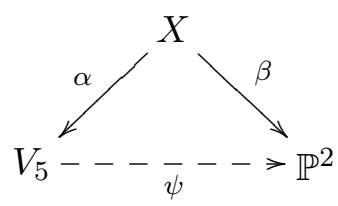

where $V_{5} \subset \mathbb{P}^{6}$ is a smooth intersection of $\operatorname{Gr}(2,5) \subset \mathbb{P}^{9}$ with a linear subspace of dimension 6 , the morphism $\alpha$ is a blow up of a cubic curve $\mathbb{P}^{1} \cong C \subset V_{5}$, the morphism $\beta$ is a conic bundle, and $\psi$ is given by the linear system of surfaces in $\left|\mathcal{O}_{\mathbb{P}^{6}}(1)\right|_{V_{5}} \mid$ that contain the curve $C$. One has

$$
-K_{X} \sim 2 S+E
$$

where $S \in\left|\beta^{*}\left(\mathcal{O}_{\mathbb{P}^{2}}(1)\right)\right|$, and $E$ is the exceptional divisor of $\alpha$. We see that $\operatorname{lct}(X) \leqslant 1 / 2$.

Lemma 11.13. Suppose that $I(X)=2.21$. Then $\operatorname{lct}(X) \leqslant 2 / 3$.

Proof. There is a commutative diagram

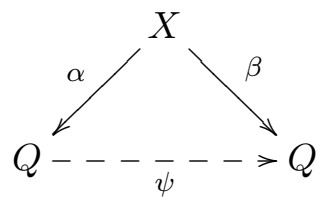

where $Q \subset \mathbb{P}^{4}$ is a smooth quadric threefold, the morphisms $\alpha$ and $\beta$ are blow ups of smooth rational curves $C \subset Q$ and $Z \subset Q$ of degree 4 , and $\psi$ is a birational map that is given by the linear system of surfaces in $\left|\mathcal{O}_{\mathbb{P}^{4}}(2)\right|_{Q} \mid$ that contain the curve $C$. One has

$$
-K_{X} \sim \mathbb{Q} \frac{3}{2} S+\frac{1}{2} E
$$

where $S \in\left|\beta^{*}\left(\mathcal{O}_{\mathbb{P}^{4}}(1)\right)\right|_{Q} \mid$, and $E$ is the exceptional divisor of $\alpha$. We see that $\operatorname{lct}(X) \leqslant 2 / 3$. 
Lemma 11.14. Suppose that $I(X)=2.22$. Then $\operatorname{lct}(X) \leqslant 1 / 2$.

Proof. There is a commutative diagram

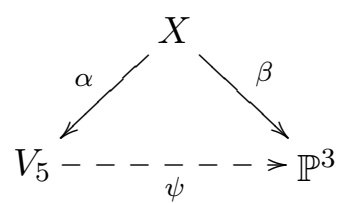

where $V_{5} \subset \mathbb{P}^{6}$ is a smooth intersection of $\operatorname{Gr}(2,5) \subset \mathbb{P}^{9}$ with a linear subspace of dimension 6 , the morphisms $\alpha$ and $\beta$ are blow ups of a conic $C \subset V_{5}$ and a rational (not linearly normal) quartic $Z \subset \mathbb{P}^{3}$, respectively, and $\psi$ is given by the linear system of surfaces in $\left|\mathcal{O}_{\mathbb{P}^{6}}(1)\right|_{V_{5}} \mid$ that contain the curve $C$. One has

$$
-K_{X} \sim 2 S+E
$$

where $S \in\left|\beta^{*}\left(\mathcal{O}_{\mathbb{P}^{3}}(1)\right)\right|$, and $E$ is the exceptional divisor of $\alpha$. We see that $\operatorname{lct}(X) \leqslant 1 / 2$.

Lemma 11.15. Suppose that $I(X)=3.13$. Then $\operatorname{lct}(X) \leqslant 1 / 2$.

Proof. There is a commutative diagram

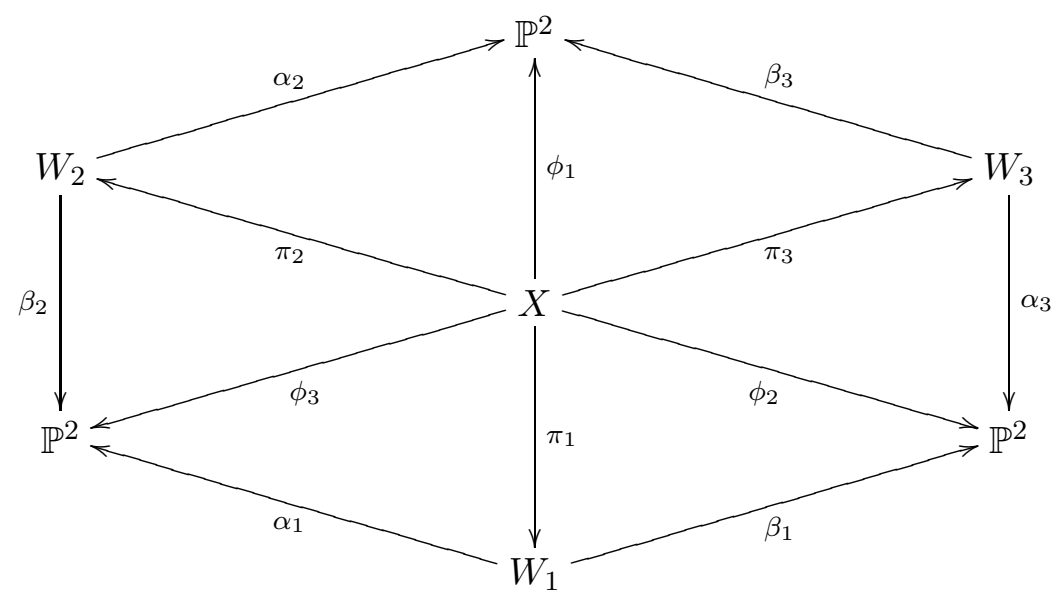

such that $W_{i} \subset \mathbb{P}^{2} \times \mathbb{P}^{2}$ is a divisor of bi-degree $(1,1)$, the morphisms $\alpha_{i}$ and $\beta_{i}$ are $\mathbb{P}^{1}$-bundles, $\pi_{i}$ is a blow up of a smooth curve $C_{i} \subset W_{i}$ of bi-degree $(2,2)$ such that

$$
\alpha_{i}\left(C_{i}\right) \subset \mathbb{P}^{2} \supset \beta_{i}\left(C_{i}\right)
$$

are irreducible conics, and $\phi_{i}$ is a conic bundle. Let $E_{i}$ be the exceptional divisor of $\pi_{i}$. Then

$$
-K_{X} \sim 2 H_{1}+E_{1} \sim 2 H_{2}+E_{2} \sim 2 H_{3}+E_{3} \sim E_{1}+E_{2}+E_{3},
$$

where $H_{i} \in\left|\phi_{i}^{*}\left(\mathcal{O}_{\mathbb{P}^{2}}(1)\right)\right|$. We see that $\operatorname{lct}(X) \leqslant 1 / 2$.

Remark 11.16. Let us use the notation of the proof of Lemma 11.15 and assume that lct $(X)<$ $1 / 2$. Then there is an effective $\mathbb{Q}$-divisor $D \sim_{\mathbb{Q}}-K_{X}$ such that the log pair $(X, \lambda D)$ is not log canonical for some $\lambda<1 / 2$. Since $\operatorname{lct}\left(W_{i}\right)=1 / 2$ by Theorem 7.1 , one has

$$
\varnothing \neq \operatorname{LCS}(X, \lambda D) \subset E_{1} \cap E_{2} \cap E_{3} .
$$

In particular, by Theorem 2.7 the locus $\operatorname{LCS}(X, \lambda D)$ consists of a single point $P$; note that $P$ is an intersection $P=F_{1} \cap F_{2} \cap F_{3}$ of three curves $F_{i}$ such that $F_{2} \cup F_{3}$ (resp., $F_{1} \cup F_{3}, F_{1} \cup F_{2}$ ) is a reducible fiber of the conic bundle $\phi_{1}$ (resp., $\phi_{2}, \phi_{3}$ ).

\section{Appendix A. By Jean-Pierre Demailly. On Tian's invariant and log Canonical THRESHOLDS}

The goal of this appendix is to relate log canonical thresholds with the $\alpha$-invariant introduced by G. Tian [179] for the study of the existence of Kähler-Einstein metrics. The approximation technique of closed positive $(1,1)$-currents introduced in [48] is used to show that the $\alpha$-invariant actually coincides with the log canonical threshold. 
Algebraic geometers have been aware of this fact after [49] appeared, and several papers have used it consistently in the latter years (see e.g. [81, [12]). However, it turns out that the required result is stated only in a local analytic form in [49], in a language which may not be easily recognizable by algebraically minded people. Therefore, we will repair here the lack of a proper reference by stating and proving the statements required for the applications to projective varieties, e.g. existence of Kähler-Einstein metrics on Fano varieties and Fano orbifolds.

Usually, in these applications, only the case of the anticanonical line bundle $L=-K_{X}$ is considered. Here we will consider more generally the case of an arbitrary line bundle $L$ (or $\mathbb{Q}$ line bundle $L$ ) on a complex manifold $X$, with some additional restrictions which will be stated later.

Assume that $L$ is equipped with a singular hermitian metric $h$ (see e.g. [47]). Locally, $L$ admits trivializations $\theta: L_{\mid U} \simeq U \times \mathbb{C}$, and on $U$ the metric $h$ is given by a weight function $\varphi$ such that

$$
\|\xi\|_{h}^{2}=|\xi|^{2} e^{-2 \varphi(z)} \text { for all } z \in U, \xi \in L_{z}
$$

when $\xi \in L_{z}$ is identified with a complex number. We are interested in the case where $\varphi$ is (at the very least) a locally integrable function for the Lebesgue measure, since it is then possible to compute the curvature form

$$
\Theta_{L, h}=\frac{i}{\pi} \partial \bar{\partial} \varphi
$$

in the sense of distributions. We have $\Theta_{L, h} \geqslant 0$ as a $(1,1)$-current if and only if the weights $\varphi$ are plurisubharmonic functions. In the sequel we will be interested only in that case.

Let us first introduce the concept of complex singularity exponent for singular hermitian metrics, following e.g. [184, [185, [4] and [49].

Definition A.1. If $K$ is a compact subset of $X$, we define the complex singularity exponent $c_{K}(h)$ of the metric $h$, written locally as $h=e^{-2 \varphi}$, to be the supremum of all positive numbers $c$ such that $h^{c}=e^{-2 c \varphi}$ is integrable in a neighborhood of every point $z_{0} \in K$, with respect to the Lebesgue measure in holomorphic coordinates centered at $z_{0}$.

Now, we introduce a generalized version of Tian's invariant $\alpha$, as defined in [179] (see also [173]).

Definition A.2. Assume that $X$ is a compact manifold and that $L$ is a pseudo-effective line bundle, i. e. $L$ admits a singular hermitian metric $h_{0}$ with $\Theta_{L, h_{0}} \geqslant 0$. If $K$ is a compact subset of $X$, we put

$$
\alpha_{K}(L)=\inf _{\left\{h, \Theta_{L, h} \geqslant 0\right\}} c_{K}(h)
$$

where $h$ runs over all singular hermitian metrics on $L$ such that $\Theta_{L, h} \geqslant 0$.

In algebraic geometry, it is more usual to look instead at linear systems defined by a family of linearly independent sections $\sigma_{0}, \sigma_{1}, \ldots, \sigma_{N} \in H^{0}\left(X, L^{\otimes m}\right)$. We denote by $\Sigma$ the vector subspace generated by these sections and by

$$
|\Sigma|:=P(\Sigma) \subset|m L|:=P\left(H^{0}\left(X, L^{\otimes m}\right)\right)
$$

the corresponding linear system. Such an $(N+1)$-tuple of sections $\sigma=\left(\sigma_{j}\right)_{0 \leqslant j \leqslant N}$ defines a singular hermitian metric $h$ on $L$ by putting in any trivialization

$$
\|\xi\|_{h}^{2}=\frac{|\xi|^{2}}{\left(\sum_{j}\left|\sigma_{j}(z)\right|^{2}\right)^{1 / m}}=\frac{|\xi|^{2}}{|\sigma(z)|^{2 / m}} \text { for } \xi \in L_{z}
$$

hence $h(z)=|\sigma(z)|^{-2 / m}$ with

$$
\varphi(z)=\frac{1}{m} \log |\sigma(z)|=\frac{1}{2 m} \log \sum_{j}\left|\sigma_{j}(z)\right|^{2}
$$

as the associated weight function. Therefore, we are interested in the number $c_{K}\left(|\sigma|^{-2 / m}\right)$. In the case of a single section $\sigma_{0}$ (corresponding to a linear system containing a single divisor), this is the same as the $\log$ canonical threshold $\operatorname{lct}_{K}\left(X, \frac{1}{m} D\right)$ of the where $D$ is a divisor corresponding 
to $\sigma_{0}$. We will also use the formal notation $\operatorname{lct}_{K}\left(X, \frac{1}{m}|\Sigma|\right)$ in the case of a higher dimensional linear system $|\Sigma| \subset|m L|$.

Now, recall that the line bundle $L$ is said to be big if the Kodaira-Iitaka dimension $\varkappa(L)$ equals $n=\operatorname{dim}_{\mathbb{C}}(X)$. The main result of this appendix is

Theorem A.3. Let $L$ be a big line bundle on a compact complex manifold $X$. Then for every compact set $K$ in $X$ we have

$$
\alpha_{K}(L)=\inf _{\left\{h, \Theta_{L, h} \geqslant 0\right\}} c_{K}(h)=\inf _{m \in \mathbb{Z}>0} \inf _{D \in|m L|} \operatorname{lct}_{K}\left(X, \frac{1}{m} D\right) .
$$

Observe that the inequality

$$
\inf _{m \in \mathbb{Z}>0} \inf _{D \in|m L|} \operatorname{lct}_{K}\left(X, \frac{1}{m} D\right) \geq \inf _{\left\{h, \Theta_{L, h} \geqslant 0\right\}} c_{K}(h)
$$

is trivial, since any divisor $D \in|m L|$ gives rise to a singular hermitian metric $h$. The converse inequality will follow from the approximation technique of [48] and some elementary analysis. The proof is parallel to the proof of [49, Theorem 4.2], although the language used there was somewhat different. In any case, we use again the crucial concept of multiplier ideal sheaves: if $h$ is a singular hermitian metric with local plurisubharmonic weights $\varphi$, the multiplier ideal sheaf $\mathcal{I}(h) \subset \mathcal{O}_{X}$ (also denoted by $\mathcal{I}(\varphi)$ ) is the ideal sheaf defined by

$$
\begin{aligned}
& \mathcal{I}(h)_{z}=\left\{\begin{array}{l}
f \in \mathcal{O}_{X, z} \mid \text { there exists a neighborhood } V \ni z,
\end{array}\right. \\
& \left\{\text { such that } \int_{V}|f(x)|^{2} e^{-2 \varphi(x)} d \lambda(x)<+\infty\right\},
\end{aligned}
$$

where $\lambda$ is the Lebesgue measure. By Nadel (see [132]), this is a coherent analytic sheaf on $X$. Theorem A.3 has a more precise version which can be stated as follows.

Theorem A.4. Let $L$ be a line bundle on a compact complex manifold $X$ possessing a singular hermitian metric $h$ with $\Theta_{L, h} \geqslant \varepsilon \omega$ for some $\varepsilon>0$ and some smooth positive definite hermitian $(1,1)$-form $\omega$ on $X$. For every real number $m>0$, consider the space $\mathcal{H}_{m}=H^{0}\left(X, L^{\otimes m} \otimes \mathcal{I}\left(h^{m}\right)\right)$ of holomorphic sections $\sigma$ of $L^{\otimes m}$ on $X$ such that

$$
\int_{X}|\sigma|_{h^{m}}^{2} d V_{\omega}=\int_{X}|\sigma|^{2} e^{-2 m \varphi} d V_{\omega}<+\infty
$$

where $d V_{\omega}=\frac{1}{m !} \omega^{m}$ is the hermitian volume form. Then for $m \gg 1, \mathcal{H}_{m}$ is a non zero finite dimensional Hilbert space and we consider the closed positive $(1,1)$-current

$$
T_{m}=\frac{i}{2 \pi} \partial \bar{\partial}\left(\frac{1}{2 m} \log \sum_{k}\left|g_{m, k}\right|^{2}\right)=\frac{i}{2 \pi} \partial \bar{\partial}\left(\frac{1}{2 m} \log \sum_{k}\left|g_{m, k}\right|_{h}^{2}\right)+\Theta_{L, h}
$$

where $\left(g_{m, k}\right)_{1 \leqslant k \leqslant N(m)}$ is an orthonormal basis of $\mathcal{H}_{m}$. The following statements hold.

(i) For every trivialization $L_{\mid U} \simeq U \times \mathbb{C}$ on a cordinate open set $U$ of $X$ and every compact set $K \subset U$, there are constants $C_{1}, C_{2}>0$ independent of $m$ and $\varphi$ such that

$$
\varphi(z)-\frac{C_{1}}{m} \leqslant \psi_{m}(z):=\frac{1}{2 m} \log \sum_{k}\left|g_{m, k}(z)\right|^{2} \leqslant \sup _{|x-z|<r} \varphi(x)+\frac{1}{m} \log \frac{C_{2}}{r^{n}}
$$

for every $z \in K$ and $r \leqslant \frac{1}{2} d(K, \partial U)$. In particular, $\psi_{m}$ converges to $\varphi$ pointwise and in $L_{\text {loc }}^{1}$ topology on $\Omega$ when $m \rightarrow+\infty$, hence $T_{m}$ converges weakly to $T=\Theta_{L, h}$.

(ii) The Lelong numbers $\nu(T, z)=\nu(\varphi, z)$ and $\nu\left(T_{m}, z\right)=\nu\left(\psi_{m}, z\right)$ are related by

$$
\nu(T, z)-\frac{n}{m} \leqslant \nu\left(T_{m}, z\right) \leqslant \nu(T, z) \quad \text { for every } z \in X .
$$

(iii) For every compact set $K \subset X$, the complex singularity exponents of the metrics given locally by $h=e^{-2 \varphi}$ and $h_{m}=e^{-2 \psi_{m}}$ satisfy

$$
c_{K}(h)^{-1}-\frac{1}{m} \leqslant c_{K}\left(h_{m}\right)^{-1} \leqslant c_{K}(h)^{-1} .
$$


Proof. The major part of the proof is a small variation of the arguments already explained in [48] (see also [49, Theorem 4.2]). We give them here in some detail for the convenience of the reader.

(i) We note that $\sum\left|g_{m, k}(z)\right|^{2}$ is the square of the norm of the evaluation linear form $\sigma \mapsto \sigma(z)$ on $\mathcal{H}_{m}$, hence

$$
\psi_{m}(z)=\sup _{\sigma \in B(1)} \frac{1}{m} \log |\sigma(z)|
$$

where $B(1)$ is the unit ball of $\mathcal{H}_{m}$. For $r \leqslant \frac{1}{2} d(K, \partial \Omega)$, the mean value inequality applied to the plurisubharmonic function $|\sigma|^{2}$ implies

$$
\begin{aligned}
|\sigma(z)|^{2} \leqslant \frac{1}{\pi^{n} r^{2 n} / n !} \int_{|x-z|<r}|\sigma(x)|^{2} d \lambda(x) & \leqslant \\
& \leqslant \frac{1}{\pi^{n} r^{2 n} / n !} \exp \left(2 m \sup _{|x-z|<r} \varphi(x)\right) \int_{\Omega}|\sigma|^{2} e^{-2 m \varphi} d \lambda .
\end{aligned}
$$

If we take the supremum over all $\sigma \in B(1)$ we get

$$
\psi_{m}(z) \leqslant \sup _{|x-z|<r} \varphi(x)+\frac{1}{2 m} \log \frac{1}{\pi^{n} r^{2 n} / n !}
$$

and the right hand inequality in (i) is proved. Conversely, the Ohsawa-Takegoshi extension theorem [134], 135] applied to the 0-dimensional subvariety $\{z\} \subset U$ shows that for any $a \in \mathbb{C}$ there is a holomorphic function $f$ on $U$ such that $f(z)=a$ and

$$
\int_{U}|f|^{2} e^{-2 m \varphi} d \lambda \leqslant C_{3}|a|^{2} e^{-2 m \varphi(z)}
$$

where $C_{3}$ only depends on $n$ and $\operatorname{diam}(U)$. Now, provided $a$ remains in a compact set $K \subset U$, we can use a cut-off function $\theta$ with support in $U$ and equal to 1 in a neighborhood of $a$, and solve the $\bar{\partial}$-equation $\bar{\partial} g=\bar{\partial}(\theta f)$ in the $L^{2}$ space associated with the weight $2 m \varphi+2(n+1) \log |z-a|$, that is, the singular hermitian metric $h(z)^{m}|z-a|^{-2(n+1)}$ on $L^{\otimes m}$. For this, we apply the standard Andreotti-Vesentini-Hörmander $L^{2}$ estimates (see e.g. [46] for the required version). This is possible for $m \geqslant m_{0}$ thanks to the hypothesis that $\Theta_{L, h} \geqslant \varepsilon \omega>0$, even if $X$ is non Kähler ( $X$ is in any event a Moishezon variety from our assumptions). The bound $m_{0}$ depends only on $\varepsilon$ and the geometry of a finite covering of $X$ by compact sets $K_{j} \subset U_{j}$, where $U_{j}$ are coordinate balls (say); it is independent of the point $a$ and even of the metric $h$. It follows that $g(a)=0$ and therefore $\sigma=\theta f-g$ is a holomorphic section of $L^{\otimes m}$ such that

$$
\int_{X}|\sigma|_{h^{m}}^{2} d V_{\omega}=\int_{X}|\sigma|^{2} e^{-2 m \varphi} d V_{\omega} \leqslant C_{4} \int_{U}|f|^{2} e^{-2 m \varphi} d V_{\omega} \leqslant C_{5}|a|^{2} e^{-2 m \varphi(z)}
$$

in particular, $\sigma \in \mathcal{H}_{m}=H^{0}\left(X, L^{\otimes m} \otimes \mathcal{I}\left(h^{m}\right)\right)$. We fix a such that the right hand side of the latter inequality is 1 . This gives the inequality

$$
\psi_{m}(z) \geqslant \frac{1}{m} \log |a|=\varphi(z)-\frac{\log C_{5}}{2 m}
$$

which is the left hand part of statement (i).

(ii) The first inequality in (i) implies $\nu\left(\psi_{m}, z\right) \leqslant \nu(\varphi, z)$. In the opposite direction, we find

$$
\sup _{|x-z|<r} \psi_{m}(x) \leqslant \sup _{|x-z|<2 r} \varphi(x)+\frac{1}{m} \log \frac{C_{2}}{r^{n}} .
$$

Divide by $\log r<0$ and take the limit as $r$ tends to 0 . The quotient by $\log r$ of the supremum of a psh function over $B(x, r)$ tends to the Lelong number at $x$. Thus we obtain

$$
\nu\left(\psi_{m}, x\right) \geqslant \nu(\varphi, x)-\frac{n}{m} .
$$

(iii) Again, the first inequality in (i) immediately yields $h_{m} \leqslant C_{6} h$, hence $c_{K}\left(h_{m}\right) \geqslant c_{K}(h)$. For the converse inequality, since we have $c_{\cup K_{j}}(h)=\min _{j} c_{K_{j}}(h)$, we can assume without loss of generality that $K$ is contained in a trivializing open patch $U$ of $L$. Let us take $c<c_{K}\left(\psi_{m}\right)$. Then, 
by definition, if $V \subset X$ is a sufficiently small open neighborhood of $K$, the Hölder inequality for the conjugate exponents $p=1+m c^{-1}$ and $q=1+m^{-1} c$ implies, thanks to equality $\frac{1}{p}=\frac{c}{m q}$,

$$
\begin{gathered}
\int_{V} e^{-2(m / p) \varphi} d V_{\omega}=\int_{V}\left(\sum_{1 \leqslant k \leqslant N(m)}\left|g_{m, k}\right|^{2} e^{-2 m \varphi}\right)^{1 / p}\left(\sum_{1 \leqslant k \leqslant N(m)}\left|g_{m, k}\right|^{2}\right)^{-c / m q} d V_{\omega} \leqslant \\
\leqslant\left(\int_{X} \sum_{1 \leqslant k \leqslant N(m)}\left|g_{m, k}\right|^{2} e^{-2 m \varphi} d V_{\omega}\right)^{1 / p}\left(\int_{V}\left(\sum_{1 \leqslant k \leqslant N(m)}\left|g_{m, k}\right|^{2}\right)^{-c / m} d V_{\omega}\right)^{1 / q}= \\
=N(m)^{1 / p}\left(\int_{V}\left(\sum_{1 \leqslant k \leqslant N(m)}\left|g_{m, k}\right|^{2}\right)^{-c / m} d V_{\omega}\right)^{1 / q}<+\infty .
\end{gathered}
$$

From this we infer $c_{K}(h) \geqslant m / p$, i.e., $c_{K}(h)^{-1} \leqslant p / m=1 / m+c^{-1}$. As $c<c_{K}\left(\psi_{m}\right)$ was arbitrary, we get $c_{K}(h)^{-1} \leqslant 1 / m+c_{K}\left(h_{m}\right)^{-1}$ and the inequalities of (iii) are proved.

Proof of Theorem A.3. Given a big line bundle $L$ on $X$, there exists a modification $\mu: \tilde{X} \rightarrow X$ of $X$ such that $\tilde{X}$ is projective and $\mu^{*} L=\mathcal{O}(A+E)$ where $A$ is an ample divisor and $E$ an effective divisor with rational coefficients. By pushing forward by $\mu$ a smooth metric $h_{A}$ with positive curvature on $A$, we get a singular hermitian metric $h_{1}$ on $L$ such that

$$
\Theta_{L, h_{1}} \geqslant \mu_{*} \Theta_{A, h_{A}} \geqslant \varepsilon \omega
$$

on $X$. Then for any $\delta>0$ and any singular hermitian metric $h$ on $L$ with $\Theta_{L, h} \geqslant 0$, the interpolated metric $h_{\delta}=h_{1}^{\delta} h^{1-\delta}$ satisfies $\Theta_{L, h_{\delta}} \geqslant \delta \varepsilon \omega$. Since $h_{1}$ is bounded away from 0 , it follows that $c_{K}(h) \geqslant(1-\delta) c_{K}\left(h_{\delta}\right)$ by monotonicity. By Theorem A.4 (iii) applied to $h_{\delta}$, we infer

$$
c_{K}\left(h_{\delta}\right)=\lim _{m \rightarrow+\infty} c_{K}\left(h_{\delta, m}\right)
$$

and we also have

$$
c_{K}\left(h_{\delta, m}\right) \geqslant \operatorname{lct}_{K}\left(\frac{1}{m} D_{\delta, m}\right)
$$

for any divisor $D_{\delta, m}$ associated with a section $\sigma \in H^{0}\left(X, L^{\otimes m} \otimes \mathcal{I}\left(h_{\delta}^{m}\right)\right)$, since the metric $h_{\delta, m}$ is given by $h_{\delta, m}=\left(\sum_{k}\left|g_{m, k}\right|^{2}\right)^{-1 / m}$ for an orthornormal basis of such sections. This clearly implies

$$
c_{K}(h) \geqslant \liminf _{\delta \rightarrow 0} \liminf _{m \rightarrow+\infty} \operatorname{lct}_{K}\left(\frac{1}{m} D_{\delta, m}\right) \geqslant \inf _{m \in \mathbb{Z}_{>0}} \inf _{D \in|m L|} \operatorname{lct}_{K}\left(\frac{1}{m} D\right) .
$$

In the applications, it is frequent to have a finite or compact group $G$ of automorphisms of $X$ and to look at $G$-invariant objects, namely $G$-equivariant metrics on $G$-equivariant line bundles $L$; in the case of a reductive algebraic group $G$ we simply consider a compact real form $G^{\mathbb{R}}$ instead of $G$ itself.

One then gets an $\alpha$ invariant $\alpha_{G, K}(L)$ by looking only at $G$-equivariant metrics in Definition A.2. All contructions made are then $G$-equivariant, especially $\mathcal{H}_{m} \subset|m L|$ is a $G$-invariant linear system. For every $G$-invariant compact set $K$ in $X$, we thus infer

$$
\alpha_{G, K}(L)=\inf _{\left\{h \text { is } G \text {-equivariant, } \Theta_{L, h} \geqslant 0\right\}} c_{K}(h)=\inf _{m \in \mathbb{Z}_{>0}} \inf _{|\Sigma| \subset|m L|, \Sigma^{G}=\Sigma} \operatorname{lct}_{K}\left(\frac{1}{m}|\Sigma|\right) .
$$

When $G$ is a finite group, one can pick for $m$ large enough a $G$-invariant divisor $D_{\delta, m}$ associated with a $G$-invariant section $\sigma$, possibly after multiplying $m$ by the order of $G$. One then gets the slightly simpler equality

$$
\alpha_{G, K}(L)=\inf _{m \in \mathbb{Z}>0} \inf _{D \in|m L|^{G}} \operatorname{lct}_{K}\left(\frac{1}{m} D\right) .
$$

In a similar manner, one can work on an orbifold $X$ rather than on a non singular variety. The $L^{2}$ techniques work in this setting with almost no change ( $L^{2}$ estimates are essentially insensitive to singularities, since one can just use an orbifold metric on the open set of regular points). 


\section{Appendix B. The Big Table}

This appendix contains the list of nonsingular Fano threefolds. We follow the notation and the numeration of these in [98, [126], [127]. We also assume the following conventions

- the symbol $V_{i}$ denotes a smooth Fano threefold such that $-K_{X} \sim 2 H$ and

$$
\operatorname{Pic}\left(V_{i}\right)=\mathbb{Z}[H],
$$

where $H$ is a Cartier divisor on $V_{i}$, and $H^{3}=8 i \in\{8,16, \ldots, 40\}$,

- the symbol $W$ denotes a divisor on $\mathbb{P}^{2} \times \mathbb{P}^{2}$ of bidegree $(1,1)$ (or, that is the same, the variety $\mathbb{P}\left(T_{\mathbb{P}^{2}}\right)$ ),

- the symbol $V_{7}$ denotes a blow up of $\mathbb{P}^{3}$ at a point (or, that is the same, the variety $\left.\mathbb{P}\left(\mathcal{O}_{\mathbb{P}^{2}} \oplus \mathcal{O}_{\mathbb{P}^{2}}(1)\right)\right)$,

- the symbol $Q$ denotes a smooth quadric hypersurface in $\mathbb{P}^{4}$,

- the symbol $S_{i}$ denotes a smooth del Pezzo surface such that

$$
K_{S_{i}}^{2}=i \in\{1, \ldots, 8\},
$$

where $S_{8} \approx \mathbb{P}^{1} \times \mathbb{P}^{1}$.

The fourth column of Table 1 contains the values of global log canonical thresholds of the corresponding Fano varieties. The symbol $\star$ near a number means that $\operatorname{lct}(X)$ is calculated for a general $X$ with a given deformation type. If we know only the upper bound $\operatorname{lct}(X) \leqslant \alpha$, we write $\leqslant \alpha$ instead of the exact value of lct $(X)$, and the symbol ? means that we don't know any reasonable upper bound (apart from a trivial lct $(X) \leqslant 1$ ).

\begin{tabular}{|c|c|c|c|}
\hline$\exists(X)$ & $-K_{X}^{3}$ & Brief description & $\operatorname{lct}(X)$ \\
\hline 1.1 & 2 & a hypersurface in $\mathbb{P}(1,1,1,1,3)$ of degree 6 & $1 \star$ \\
\hline 1.2 & 4 & $\begin{array}{l}\text { a hypersurface in } \mathbb{P}^{4} \text { of degree } 4 \text { or } \\
\text { a double cover of smooth quadric in } \mathbb{P}^{4} \text { branched over a surface of degree } 8\end{array}$ & $?$ \\
\hline 1.3 & 6 & a complete intersection of a quadric and a cubic in $\mathbb{P}^{5}$ & $?$ \\
\hline 1.4 & 8 & a complete intersection of three quadrics $\mathbb{P}^{6}$ & $?$ \\
\hline 1.5 & 10 & a section of $\operatorname{Gr}(2,5) \subset \mathbb{P}^{9}$ by quadric and linear subspace of dimension 7 & $?$ \\
\hline 1.6 & 12 & $\begin{array}{l}\text { a section of the Hermitian symmetric space } M=G / P \subset \mathbb{P}^{15} \\
\text { of type DIII by a linear subspace of dimension } 8\end{array}$ & $?$ \\
\hline 1.7 & 14 & a section of $\operatorname{Gr}(2,6) \subset \mathbb{P}^{14}$ by a linear subspace of codimension 5 & $?$ \\
\hline 1.8 & 16 & $\begin{array}{l}\text { a section of the Hermitian symmetric space } M=G / P \subset \mathbb{P}^{19} \\
\text { of type CI by a linear subspace of dimension } 10\end{array}$ & $\leqslant 6 / 7$ \\
\hline 1.9 & 18 & $\begin{array}{l}\text { a section of the } 5 \text {-dimensional rational homogeneous contact } \\
\text { manifold } G_{2} / P \subset \mathbb{P}^{13} \text { by a linear subspace of dimension } 11\end{array}$ & $\leqslant 4 / 5$ \\
\hline 1.10 & 22 & $\begin{array}{l}\text { a zero locus of three sections of the rank } 3 \text { vector bundle } \wedge^{2} \mathcal{Q} \\
\text { where } \mathcal{Q} \text { is the universal quotient bundle on } \operatorname{Gr}(7,3)\end{array}$ & $\leqslant 2 / 3$ \\
\hline 1.11 & 8 & $V_{1}$ that is a hypersurface in $\mathbb{P}(1,1,1,2,3)$ of degree 6 & $1 / 2$ \\
\hline 1.12 & 16 & $V_{2}$ that is a hypersurface in $\mathbb{P}(1,1,1,1,2)$ of degree 4 & $1 / 2$ \\
\hline 1.13 & 24 & $V_{3}$ that is a hypersurface in $\mathbb{P}^{4}$ of degree 3 & $1 / 2$ \\
\hline 1.14 & 32 & $V_{4}$ that is a complete intersection of two quadrics in $\mathbb{P}^{5}$ & $1 / 2$ \\
\hline 1.15 & 40 & $V_{5}$ that is a section of $\operatorname{Gr}(2,5) \subset \mathbb{P}^{9}$ by linear subspace of codimension 3 & $1 / 2$ \\
\hline 1.16 & 54 & $Q$ that is a hypersurface in $\mathbb{P}^{4}$ of degree 2 & $1 / 3$ \\
\hline
\end{tabular}

Table 1: Smooth Fano threefolds 


\begin{tabular}{|c|c|c|c|}
\hline 1.17 & 64 & $\mathbb{P}^{3}$ & $1 / 4$ \\
\hline 2.1 & 4 & $\begin{array}{l}\text { a blow up of the Fano threefold } V_{1} \text { along an elliptic curve } \\
\text { that is an intersection of two divisors from }\left|-\frac{1}{2} K_{V_{1}}\right|\end{array}$ & $1 / 2$ \\
\hline 2.2 & 6 & a double cover of $\mathbb{P}^{1} \times \mathbb{P}^{2}$ whose branch locus is a divisor of bidegree $(2,4)$ & $\leqslant 13 / 14$ \\
\hline 2.3 & 8 & $\begin{array}{l}\text { the blow up of the Fano threefold } V_{2} \text { along an elliptic curve } \\
\text { that is an intersection of two divisors from }\left|-\frac{1}{2} K_{V_{2}}\right|\end{array}$ & $1 / 2$ \\
\hline 2.4 & 10 & the blow up of $\mathbb{P}^{3}$ along an intersection of two cubics & $3 / 4 \star$ \\
\hline 2.5 & 12 & the blow up of the threefold $V_{3} \subset \mathbb{P}^{4}$ along a plane cubic & $1 / 2 \star$ \\
\hline 2.6 & 12 & $\begin{array}{l}\text { a divisor on } \mathbb{P}^{2} \times \mathbb{P}^{2} \text { of bidegree }(2,2) \text { or } \\
\text { a double cover of } W \text { whose branch locus is a surface in }\left|-K_{W}\right|\end{array}$ & $?$ \\
\hline 2.7 & 14 & the blow up of $Q$ along the intersection of two divisors from $\left|\mathcal{O}_{Q}(2)\right|$ & $\leqslant 2 / 3$ \\
\hline 2.8 & 14 & a double cover of $V_{7}$ whose branch locus is a surface in $\left|-K_{V_{7}}\right|$ & $1 / 2 \star$ \\
\hline 2.9 & 16 & $\begin{array}{l}\text { the blow up of } \mathbb{P}^{3} \text { along a curve of degree } 7 \text { and genus } 5 \\
\text { which is an intersection of cubics }\end{array}$ & $\leqslant 3 / 4$ \\
\hline 2.10 & 16 & $\begin{array}{l}\text { the blow up of } V_{4} \subset \mathbb{P}^{5} \text { along an elliptic curve } \\
\text { which is an intersection of two hyperplane sections }\end{array}$ & $1 / 2 \star$ \\
\hline 2.11 & 18 & the blow up of $V_{3}$ along a line & $1 / 2 \star$ \\
\hline 2.12 & 20 & $\begin{array}{l}\text { the blow up of } \mathbb{P}^{3} \text { along a curve of degree } 6 \text { and genus } 3 \\
\text { which is an intersection of cubics }\end{array}$ & $\leqslant 3 / 4$ \\
\hline 2.13 & 20 & the blow up of $Q \subset \mathbb{P}^{4}$ along a curve of degree 6 and genus 2 & $\leqslant 2 / 3$ \\
\hline 2.14 & 20 & $\begin{array}{l}\text { the blow up of } V_{5} \subset \mathbb{P}^{6} \text { along an elliptic curve } \\
\text { which is an intersection of two hyperplane sections }\end{array}$ & $1 / 2 \star$ \\
\hline 2.15 & 22 & the blow up of $\mathbb{P}^{3}$ along the intersection of a quadric and a cubic surfaces & $1 / 2 \star$ \\
\hline 2.16 & 22 & the blow up of $V_{4} \subset \mathbb{P}^{5}$ along a conic & $\leqslant 1 / 2$ \\
\hline 2.17 & 24 & the blow up of $Q \subset \mathbb{P}^{4}$ along an elliptic curve of degree 5 & $\leqslant 2 / 3$ \\
\hline 2.18 & 24 & a double cover of $\mathbb{P}^{1} \times \mathbb{P}^{2}$ whose branch locus is a divisor of bidegree $(2,2)$ & $1 / 2$ \\
\hline 2.19 & 26 & the blow up of $V_{4} \subset \mathbb{P}^{5}$ along a line & $1 / 2 \star$ \\
\hline 2.20 & 26 & the blow up of $V_{5} \subset \mathbb{P}^{6}$ along a twisted cubic & $\leqslant 1 / 2$ \\
\hline 2.21 & 28 & the blow up of $Q \subset \mathbb{P}^{4}$ along a twisted quartic & $\leqslant 2 / 3$ \\
\hline 2.22 & 30 & the blow up of $V_{5} \subset \mathbb{P}^{6}$ along a conic & $\leqslant 1 / 2$ \\
\hline 2.23 & 30 & $\begin{array}{l}\text { the blow up of } Q \subset \mathbb{P}^{4} \text { along a curve of degree } 4 \text { that is an intersection of a } \\
\text { surface in }\left|\mathcal{O}_{\mathbb{P}^{4}}(1)\right|_{Q} \mid \text { and a surface in }\left|\mathcal{O}_{\mathbb{P}^{4}}(2)\right|_{Q} \mid\end{array}$ & $1 / 3 \star$ \\
\hline 2.24 & 30 & a divisor on $\mathbb{P}^{2} \times \mathbb{P}^{2}$ of bidegree $(1,2)$ & $1 / 2 \star$ \\
\hline 2.25 & 32 & the blow up of $\mathbb{P}^{3}$ along an elliptic curve which is an intersection of two quadrics & $1 / 2$ \\
\hline 2.26 & 34 & the blow up of the threefold $V_{5} \subset \mathbb{P}^{6}$ along a line & $1 / 2 \star$ \\
\hline 2.27 & 38 & the blow up of $\mathbb{P}^{3}$ along a twisted cubic & $1 / 2$ \\
\hline 2.28 & 40 & the blow up of $\mathbb{P}^{3}$ along a plane cubic & $1 / 4$ \\
\hline 2.29 & 40 & the blow up of $Q \subset \mathbb{P}^{4}$ along a conic & $1 / 3$ \\
\hline 2.30 & 46 & the blow up of $\mathbb{P}^{3}$ along a conic & $1 / 4$ \\
\hline
\end{tabular}




\begin{tabular}{|c|c|c|c|}
\hline 2.31 & 46 & the blow up of $Q \subset \mathbb{P}^{4}$ along a line & $1 / 3$ \\
\hline 2.32 & 48 & $W$ that is a divisor on $\mathbb{P}^{2} \times \mathbb{P}^{2}$ of bidegree $(1,1)$ & $1 / 2$ \\
\hline 2.33 & 54 & the blow up of $\mathbb{P}^{3}$ along a line & $1 / 4$ \\
\hline 2.34 & 54 & $\mathbb{P}^{1} \times \mathbb{P}^{2}$ & $1 / 3$ \\
\hline 2.35 & 56 & $V_{7} \cong \mathbb{P}\left(\mathcal{O}_{\mathbb{P}^{2}} \oplus \mathcal{O}_{\mathbb{P}^{2}}(1)\right)$ & $1 / 4$ \\
\hline 2.36 & 62 & $\mathbb{P}\left(\mathcal{O}_{\mathbb{P}^{2}} \oplus \mathcal{O}_{\mathbb{P}^{2}}(2)\right)$ & $1 / 5$ \\
\hline 3.1 & 12 & a double cover of $\mathbb{P}^{1} \times \mathbb{P}^{1} \times \mathbb{P}^{1}$ branched in a divisor of tridegree $(2,2,2)$ & $3 / 4 \star$ \\
\hline 3.2 & 14 & $\begin{array}{l}\text { a divisor on a } \mathbb{P}^{2} \text {-bundle } \mathbb{P}\left(\mathcal{O}_{\mathbb{P}^{1}} \times \mathbb{P}^{1} \oplus \mathcal{O}_{\mathbb{P}^{1} \times \mathbb{P}^{1}}(-1,-1) \oplus \mathcal{O}_{\mathbb{P}^{1}} \times \mathbb{P}^{1}(-1,-1)\right) \\
\text { such that } X \in\left|L^{\otimes 2} \otimes \mathcal{O}_{\mathbb{P}^{1} \times \mathbb{P}^{1}}(2,3)\right| \text {, where } L \text { is the tautological line bundle }\end{array}$ & $1 / 2 \star$ \\
\hline 3.3 & 18 & a divisor on $\mathbb{P}^{1} \times \mathbb{P}^{1} \times \mathbb{P}^{2}$ of tridegree $(1,1,2)$ & $2 / 3 \star$ \\
\hline 3.4 & 18 & $\begin{array}{l}\text { the blow up of the Fano threefold } Y \text { with } I(Y)=2.18 \text { along a smooth fiber } \\
\text { of the composition } Y \rightarrow \mathbb{P}^{1} \times \mathbb{P}^{2} \rightarrow \mathbb{P}^{2} \text { of the double cover with the projection }\end{array}$ & $1 / 2$ \\
\hline 3.5 & 20 & $\begin{array}{l}\text { the blow up of } \mathbb{P}^{1} \times \mathbb{P}^{2} \text { along a curve } C \text { of bidegree }(5,2) \\
\text { such that the composition } C \hookrightarrow \mathbb{P}^{1} \times \mathbb{P}^{2} \rightarrow \mathbb{P}^{2} \text { is an embedding }\end{array}$ & $1 / 2 \star$ \\
\hline 3.6 & 22 & the blow up of $\mathbb{P}^{3}$ along a disjoint union of a line and an elliptic curve of degree 4 & $1 / 2 \star$ \\
\hline 3.7 & 24 & $\begin{array}{l}\text { the blow up of the threefold } W \text { along an elliptic curve } \\
\text { that is an intersection of two divisors from }\left|-\frac{1}{2} K_{W}\right|\end{array}$ & $1 / 2 \star$ \\
\hline 3.8 & 24 & $\begin{array}{l}\text { a divisor in }\left|\left(\alpha \circ \pi_{1}\right)^{*}\left(\mathcal{O}_{\mathbb{P}^{2}}(1)\right) \otimes \pi_{2}^{*}\left(\mathcal{O}_{\mathbb{P}^{2}}(2)\right)\right|, \text { where } \pi_{1}: \mathbb{F}_{1} \times \mathbb{P}^{2} \rightarrow \mathbb{F}_{1} \\
\text { and } \pi_{2}: \mathbb{F}_{1} \times \mathbb{P}^{2} \rightarrow \mathbb{P}^{2} \text { are projections, and } \alpha: \mathbb{F}_{1} \rightarrow \mathbb{P}^{2} \text { is a blow up of a point }\end{array}$ & $1 / 2 \star$ \\
\hline 3.9 & 26 & $\begin{array}{l}\text { the blow up of a cone } W_{4} \subset \mathbb{P}^{6} \text { over the Veronese surface } R_{4} \subset \mathbb{P}^{5} \\
\text { with center in a disjoint union of the vertex and a quartic on } R_{4} \cong \mathbb{P}^{2}\end{array}$ & $1 / 3$ \\
\hline 3.10 & 26 & the blow up of $Q \subset \mathbb{P}^{4}$ along a disjoint union of two conics & $1 / 2$ \\
\hline 3.11 & 28 & $\begin{array}{l}\text { the blow up of the threefold } V_{7} \text { along an elliptic curve } \\
\text { that is an intersection of two divisors from }\left|-\frac{1}{2} K_{V_{7}}\right|\end{array}$ & $1 / 2$ \\
\hline 3.12 & 28 & the blow up of $\mathbb{P}^{3}$ along a disjoint union of a line and a twisted cubic & $1 / 2$ \\
\hline 3.13 & 30 & $\begin{array}{l}\text { the blow up of } W \subset \mathbb{P}^{2} \times \mathbb{P}^{2} \text { along a curve } C \text { of bidegree }(2,2) \\
\text { such that } \pi_{1}(C) \subset \mathbb{P}^{2} \text { and } \pi_{2}(C) \subset \mathbb{P}^{2} \text { are irreducible conics, } \\
\text { where } \pi_{1}: W \rightarrow \mathbb{P}^{2} \text { and } \pi_{2}: W \rightarrow \mathbb{P}^{2} \text { are natural projections }\end{array}$ & $\leqslant 1 / 2$ \\
\hline 3.14 & 32 & $\begin{array}{l}\text { the blow up of } \mathbb{P}^{3} \text { along a disjoint union of a plane cubic curve that } \\
\text { is contained in a plane } \Pi \subset \mathbb{P}^{3} \text { and a point that is not contained in } \Pi\end{array}$ & $1 / 2$ \\
\hline 3.15 & 32 & the blow up of $Q \subset \mathbb{P}^{4}$ along a disjoint union of a line and a conic & $1 / 2$ \\
\hline 3.16 & 34 & $\begin{array}{l}\text { the blow up of } V_{7} \text { along a proper transform via the blow up } \alpha: V_{7} \rightarrow \mathbb{P}^{3} \\
\text { of a twisted cubic passing through the center of the blow up } \alpha\end{array}$ & $1 / 2$ \\
\hline 3.17 & 36 & a divisor on $\mathbb{P}^{1} \times \mathbb{P}^{1} \times \mathbb{P}^{2}$ of tridegree $(1,1,1)$ & $1 / 2$ \\
\hline 3.18 & 36 & the blow up of $\mathbb{P}^{3}$ along a disjoint union of a line and a conic & $1 / 3$ \\
\hline 3.19 & 38 & the blow up of $Q \subset \mathbb{P}^{4}$ at two non-collinear points & $1 / 3$ \\
\hline 3.20 & 38 & the blow up of $Q \subset \mathbb{P}^{4}$ along a disjoint union of two lines & $1 / 3$ \\
\hline 3.21 & 38 & the blow up of $\mathbb{P}^{1} \times \mathbb{P}^{2}$ along a curve of bidegree $(2,1)$ & $1 / 3$ \\
\hline 3.22 & 40 & the blow up of $\mathbb{P}^{1} \times \mathbb{P}^{2}$ along a conic in a fiber of the projection $\mathbb{P}^{1} \times \mathbb{P}^{2} \rightarrow \mathbb{P}^{1}$ & $1 / 3$ \\
\hline
\end{tabular}




\begin{tabular}{|c|c|c|c|}
\hline 3.23 & 42 & $\begin{array}{l}\text { the blow up of } V_{7} \text { along a proper transform via the blow up } \alpha: V_{7} \rightarrow \mathbb{P}^{3} \\
\text { of an irreducible conic passing through the center of the blow up } \alpha\end{array}$ & $1 / 4$ \\
\hline 3.24 & 42 & $W \times_{\mathbb{P}^{2}} \mathbb{F}_{1}$, where $W \rightarrow \mathbb{P}^{2}$ is a $\mathbb{P}^{1}$-bundle and $\mathbb{F}_{1} \rightarrow \mathbb{P}^{2}$ is the blow up & $1 / 3$ \\
\hline 3.25 & 44 & the blow up of $\mathbb{P}^{3}$ along a disjoint union of two lines & $1 / 3$ \\
\hline 3.26 & 46 & the blow up of $\mathbb{P}^{3}$ with center in a disjoint union of a point and a line & $1 / 4$ \\
\hline 3.27 & 48 & $\mathbb{P}^{1} \times \mathbb{P}^{1} \times \mathbb{P}^{1}$ & $1 / 2$ \\
\hline 3.28 & 48 & $\mathbb{P}^{1} \times \mathbb{F}_{1}$ & $1 / 3$ \\
\hline 3.29 & 50 & $\begin{array}{l}\text { the blow up of the Fano threefold } V_{7} \text { along a line in } E \cong \mathbb{P}^{2} \text {, } \\
\text { where } E \text { is the exceptional divisor of the blow up } V_{7} \rightarrow \mathbb{P}^{3}\end{array}$ & $1 / 5$ \\
\hline 3.30 & 50 & $\begin{array}{l}\text { the blow up of } V_{7} \text { along a proper transform via the blow up } \alpha: V_{7} \rightarrow \mathbb{P}^{3} \\
\text { of a line that passes through the center of the blow up } \alpha\end{array}$ & $1 / 4$ \\
\hline 3.31 & 52 & the blow up of a cone over a smooth quadric in $\mathbb{P}^{3}$ at the vertex & $1 / 3$ \\
\hline 4.1 & 24 & divisor on $\mathbb{P}^{1} \times \mathbb{P}^{1} \times \mathbb{P}^{1} \times \mathbb{P}^{1}$ of multidegree $(1,1,1,1)$ & $1 / 2$ \\
\hline 4.2 & 28 & $\begin{array}{l}\text { the blow up of the cone over a smooth quadric } S \subset \mathbb{P}^{3} \\
\text { along a disjoint union of the vertex and an elliptic curve on } S\end{array}$ & $1 / 2$ \\
\hline 4.3 & 30 & the blow up of $\mathbb{P}^{1} \times \mathbb{P}^{1} \times \mathbb{P}^{1}$ along a curve of tridegree $(1,1,2)$ & $1 / 2$ \\
\hline 4.4 & 32 & $\begin{array}{l}\text { the blow up of the smooth Fano threefold } Y \text { with } I(Y)=3.19 \\
\text { along the proper transform of a conic on the quadric } Q \subset \mathbb{P}^{4} \\
\text { that passes through the both centers of the blow up } Y \rightarrow Q\end{array}$ & $1 / 3$ \\
\hline 4.5 & 32 & $\begin{array}{l}\text { the blow up of } \mathbb{P}^{1} \times \mathbb{P}^{2} \text { along a disjoint union of } \\
\text { two irreducible curves of bidegree }(2,1) \text { and }(1,0)\end{array}$ & $3 / 7$ \\
\hline 4.6 & 34 & the blow up of $\mathbb{P}^{3}$ along a disjoint union of three lines & $1 / 2$ \\
\hline 4.7 & 36 & $\begin{array}{l}\text { the blow up of } W \subset \mathbb{P}^{2} \times \mathbb{P}^{2} \text { along a disjoint union of } \\
\text { two curves of bidegree }(0,1) \text { and }(1,0)\end{array}$ & $1 / 2$ \\
\hline 4.8 & 38 & the blow up of $\mathbb{P}^{1} \times \mathbb{P}^{1} \times \mathbb{P}^{1}$ along a curve of tridegree $(0,1,1)$ & $1 / 3$ \\
\hline 4.9 & 40 & $\begin{array}{l}\text { the blow up of the smooth Fano threefold } Y \text { with } \mathrm{J}(Y)=3.25 \\
\text { along a curve that is contracted by the blow up } Y \rightarrow \mathbb{P}^{3}\end{array}$ & $1 / 3$ \\
\hline 4.10 & 42 & $\mathbb{P}^{1} \times S_{7}$ & $1 / 3$ \\
\hline 4.11 & 44 & $\begin{array}{l}\text { the blow up of } \mathbb{P}^{1} \times \mathbb{F}_{1} \text { along a curve } C \cong \mathbb{P}^{1} \text { such that } C \text { is contained } \\
\text { in a fiber } F \cong \mathbb{F}_{1} \text { of the projection } \mathbb{P}^{1} \times \mathbb{F}_{1} \rightarrow \mathbb{P}^{1} \text { and } C \cdot C=-1 \text { on } F\end{array}$ & $1 / 3$ \\
\hline 4.12 & 46 & $\begin{array}{l}\text { the blow up of the smooth Fano threefold } Y \text { with } \mathrm{I}(Y)=2.33 \\
\text { along two curves that are contracted by the blow up } Y \rightarrow \mathbb{P}^{3}\end{array}$ & $1 / 4$ \\
\hline 4.13 & 26 & the blow up of $\mathbb{P}^{1} \times \mathbb{P}^{1} \times \mathbb{P}^{1}$ along a curve of tridegree $(1,1,3)$ & $1 / 2 \star$ \\
\hline 5.1 & 28 & $\begin{array}{l}\text { the blow up of the smooth Fano threefold } Y \text { with } \mathrm{J}(Y)=2.29 \\
\text { along three curves that are contracted by the blow up } Y \rightarrow Q\end{array}$ & $1 / 3$ \\
\hline 5.2 & 36 & $\begin{array}{l}\text { the blow up of the smooth Fano threefold } Y \text { with } \mathrm{J}(Y)=3.25 \text { along } \\
\text { two curves } C_{1} \neq C_{2} \text { that are contracted by the blow up } \phi: Y \rightarrow \mathbb{P}^{3} \\
\text { and that are contained in the same exceptional divisor of the blow up } \phi\end{array}$ & $1 / 3$ \\
\hline 5.3 & 36 & $\mathbb{P}^{1} \times S_{6}$ & $1 / 2$ \\
\hline 5.4 & 30 & $\mathbb{P}^{1} \times S_{5}$ & $1 / 2$ \\
\hline 5.5 & 24 & $\mathbb{P}^{1} \times S_{4}$ & $1 / 2$ \\
\hline
\end{tabular}




\begin{tabular}{|c|c|l|c|}
\hline 5.6 & 18 & $\mathbb{P}^{1} \times S_{3}$ & $1 / 2$ \\
\hline 5.7 & 12 & $\mathbb{P}^{1} \times S_{2}$ & $1 / 2$ \\
\hline 5.8 & 6 & $\mathbb{P}^{1} \times S_{1}$ & $1 / 2$ \\
\hline
\end{tabular}

\section{REFERENCES}

[1] V. Alexeev, OTwo two-dimensional terminations Duke Mathematical Journal 69 (1993), 527-545

[2] C. Araujo, Kähler-Einstein metrics for some quasi-smooth log del Pezzo surfaces Transactions of the American Mathematical Society 354 (2002), 4303-3312

[3] C. Arezzo, A. Ghigi, G. Pirola, Symmetries, quotients and Kähler-Einstein metrics Journal fur die Reine und Angewandte Mathematik 591 (2006), 177-200

[4] V.Arnold, S. Gusein-Zade, A. Varchenko, Singularities of differentiable maps, II Progress in Mathematics, Birkhäuser (1988)

[5] T. Aubin, Equations du type Monge-Ampère sur les variétés Kähleriennes compactes Bulletin des Sciences Mathématique 354 (2002), 4303-3312

[6] W. Barth, Two projective surfaces with many nodes, admitting the symmetries of the icosahedron Journal of Algebraic Geometry 5 (1996), 173-186

[7] V. Batyrev, E. Selivanova, Einstein-Kähler metrics on symmetric toric Fano manifolds Journal fur die Reine und Angewandte Mathematik, 512 (1999), 225-236

[8] C. Birkar, Ascending chain condition for log canonical thresholds and termination of log flips Duke Mathematical Journal 136 (2007), 173-180

[9] C. Birkar, P. Cascini, C. Hacon, J. McKernan, Existence of minimal models for varieties of log general type arXiv:math/0610203 (2006)

[10] J. Björk, Rings of differential operators North-Holland, Amsterdam (1979)

[11] C. Boyer, Sasakian geometry: the recent work of Krzysztof Galicki arXiv:0806.0373 (2008)

[12] C. Boyer, K. Galicki, J. Kollár, Einstein metrics on spheres Annals of Mathematics 162 (2005), 557-580

[13] C. Boyer, K. Galicki, M. Nakamaye, Sasakian-Einstein structures on $9 \#\left(S^{2} \times S^{3}\right)$ Transactions of the American Mathematical Society 354 (2002), 2983-2996

[14] C. Boyer, K. Galicki, M. Nakamaye, Einstein metrics on rational homology 7-spheres Annales de l'Institut Fourier 52 (2002), 1569-1584

[15] C. Boyer, K. Galicki, M. Nakamaye, On the geometry of Sasakian-Einstein 5-manifolds Mathematische Annalen 325 (2003), 485-524

[16] J. W. Bruce, C. T. C. Wall, On the classification of cubic surfaces Journal of the London Mathematical Society 19 (1979), 245-256

[17] A.-M. Castravet, Examples of Fano varieties of index one that are not birationally rigid Proceedings of the American Mathematical Society 135 (2007), 3783-3788

[18] F. Catanese, G. Ceresa, Constructing sextic surfaces with a given number d of nodes Journal of Pure and Applied Algebra 23 (1982), 1-12

[19] I. Cheltsov, On a smooth four-dimensional quintic Sbornik: Mathematics 191 (2001), 1399-1419

[20] I. Cheltsov, Log canonical thresholds on hypersurfaces Sbornik: Mathematics 192 (2001), 1241-1257

[21] I. Cheltsov, Nonrationality of fourfold complete intersection of quadric and quartic not containing plane Sbornik: Mathematics 194 (2003), 1679-1699

[22] I. Cheltsov, Birationally rigid Fano varieties Russian Mathematical Surveys 60 (2005), 875-965

[23] I. Cheltsov, Nonrational nodal quartic threefolds Pacific Journal of Mathematics 226 (2006), 65-81

[24] I. Cheltsov, Double cubics and double quartics Mathematische Zeitschrift 253 (2006), 75-86

[25] I. Cheltsov, On nodal sextic fivefold Mathematische Nachrichten 280 (2007), 1344-1353 
[26] I. Cheltsov, Elliptic structures on weighted three-dimensional Fano hypersurfaces Izvestiya: Mathematics, 71 (2007), 765-862

[27] I. Cheltsov, Fano varieties with many selfmaps Advances in Mathematics 217 (2008), 97-124

[28] I. Cheltsov, Double spaces with isolated singularities Sbornik: Mathematics 199 (2008), 291-306

[29] I. Cheltsov, Log canonical thresholds and Kähler-Einstein metrics on Fano threefold hypersurfaces Izvestiya: Mathematics, to appear

[30] I. Cheltsov, Extremal metrics on two Fano varieties Sbornik: Mathematics, to appear

[31] I. Cheltsov, Log canonical thresholds of del Pezzo surfaces Geometric and Functional Analysis, to appear

[32] I. Cheltsov, On singular cubic surfaces arXiv:0706.2666 (2007)

[33] I. Cheltsov, J. Park, Total log canonical thresholds and generalized Eckardt points Sbornik: Mathematics 193 (2002), 779789

[34] I. Cheltsov, J. Park, Sextic double solids arXiv:math.AG/0404452 (2004)

[35] I. Cheltsov, J. Park, Weighted Fano threefold hypersurfaces Journal fur die Reine und Angewandte Mathematik, 600 (2006), 81-116

[36] I. Cheltsov, J. Park, Halphen pencils on weighted Fano threefold hypersurfaces arXiv:math.AG/0607776 (2006)

[37] I. Cheltsov, J. Park, J. Won, Log canonical thresholds of certain Fano hypersurfaces arXiv:math.AG/0706.0751 (2007)

[38] H. Clemens, P. Griffiths, The intermediate Jacobian of the cubic threefold Annals of Mathematics 95 (1972), 73-100

[39] C. van Coevering, Toric surfaces and Sasakian-Einstein 5-manifolds arXiv:math/0607721 (2006)

[40] A. Corti, Factorizing birational maps of threefolds after Sarkisov Journal of Algebraic Geometry 4 (1995), 223-254

[41] A. Corti, Del Pezzo surfaces over Dedekind schemes Annals of Mathematics 144 (1996), 641-683

[42] A. Corti, Singularities of linear systems and 3-fold birational geometry L.M.S. Lecture Note Series 281 (2000), 259-312

[43] A. Corti, A.Pukhlikov, M. Reid, Fano 3-fold hypersurfaces L.M.S. Lecture Note Series 281 (2000), 175-258

[44] S. Crass, Solving the sextic by iteration: a study in complex geometry and dynamics Experimental Mathematics 8 (1999), 209-240

[45] C. Cutkosky, On Fano 3-folds Manuscripta Mathematica 64 (1989), 189-204

[46] J.-P. Demailly, Estimations $L^{2}$ pour l'opérateur $\bar{\partial}$ d'un fibré vectoriel holomorphe semi-positif au-dessus d'une variété kählérienne complète Annales Scientifiques de l'École Normale Supérieure 15 (1982), 457-511

[47] J.-P. Demailly, Singular hermitian metrics on positive line bundles Lecture Notes in Mathematics 1507, Springer-Verlag, Berlin (1992)

[48] J.-P. Demailly, Regularization of closed positive currents and Intersection Theory Journal of Algebraic Geometry 1 (1992), 361-409

[49] J.-P. Demailly, J. Kollár, Semi-continuity of complex singularity exponents and Kähler-Einstein metrics on Fano orbifolds Annales Scientifiques de l'École Normale Supérieure 34 (2001), 525-556

[50] I. Demin, Fano threefolds that can be represented as rulings over surfaces Izvestiya Akademii Nauk SSSR, Seriya Matematicheskaya 44 (1980), 963-971

[51] W. Ding, G. Tian, Kähler-Einstein metrics and the generalized Futaki invariant Inventiones Mathematicae 110 (1992), 315-335

[52] I. Dolgachev, V.Iskovskikh, Finite subgroups of the plane Cremona group arXiv:math.AG/0610595 (2006)

[53] S. Donaldson, Scalar curvature and stability of toric varieties Journal of Differential Geometry 62 (2002), 289-349 
[54] S. Donaldson, Lower bounds on the Calabi functional Journal of Differential Geometry 70 (2005), 453-472

[55] S. Donaldson, A note on the $\alpha$-invariant of the Mukai-Umemura 3-fold arXiv:math.AG/0711.4357 (2007)

[56] S. Endraß, On the divisor class group of double solids Manuscripta Mathematica 99 (1999), 341-358

[57] P. Eyssidieux, V. Guedj, A.Zeriahi, Singular Kahler-Einstein metrics arXiv:math.AG/0603431 (2006)

[58] C. Favre, J. Jonsson, Valuations and multiplier ideals Journal of the American Mathematical Society 18 (2005), 655-684

[59] T. de Fernex, L. Ein, M. Mustaţă, Bounds for log canonical thresholds with applications to birational rigidity Mathematical Research Letters 10 (2003), 219-236

[60] T. de Fernex, M. Mustaţă, Limits of log canonical thresholds arXiv:math.AG/0710.4978 (2007)

[61] W. Fulton, Introduction to toric varieties Princeton University Press (1993)

[62] M. Furushima, Singular del Pezzo surfaces and analytic compactifications of $\mathbb{C}^{3}$ Nagoya Mathematical Journal 104 (1986), 1-28

[63] M. Furushima, Complex analytic compactification of $\mathbb{C}^{3}$ Compositio Mathematica 76 (1990), 163-196

[64] M. Furushima, Mukai-Umemura's example of the Fano threefold with genus 12 as a compactification of $\mathbb{C}^{3}$ Nagoya Mathematical Journal 127 (1992), 145-165

[65] M. Furushima, A new example of a compactification of $\mathbb{C}^{3}$ Mathematische Zeitschrift 212 (1993), 1432-1823

[66] M. Furushima, Singular Fano compactifications of $\mathbb{C}^{3}$ Mathematische Zeitschrift 248 (2004), 709-723

[67] M. Furushima, N. Nakayama The family of lines on the Fano threefold $V_{5}$ Nagoya Mathematical Journal 116 (1989), 111-122

[68] A. Futaki, An obstruction to the existence of Einstein-Kähler metrics Inventiones Mathematicae 73 (1983), 437-443

[69] J. Gauntlett, D. Martelli, J. Sparks, S.-T. Yau, Obstructions to the existence of Sasaki-Einstein metrics arXiv:hep-th/0607080 (2006)

[70] A. Ghigi, J. Kollár, Kähler-Einstein metrics on orbifolds and Einstein metrics on spheres Commentarii Mathematici Helvetici, 82 (2007), 877-902

[71] M. Gizatullin, Rational G-surfaces Izvestiya Akademii Nauk SSSR, Seriya Matematicheskaya 441 (1980), 110-144

[72] M. Grinenko, On fibrations into del Pezzo surfaces Mathematical Notes 69 (2001), 499-513

[73] M. Grinenko, On fiberwise surgeries of fibrations into del Pezzo surface of degree 2 Russian Mathematical Surveys 56 (2001), 753-754

[74] L. Gruson, F. Laytimi, D. Nagaraj, On prime Fano threefolds of genus 9 International Journal of Mathematics 17 (2006), 253-261

[75] H. Hironaka, Resolution of singularities of an algebraic variety over a field of characteristic zero Annals of Mathematics 79 (1964), 109-326

[76] J. Hoffman, S. Weintraub, The Siegel modular variety of degree two and level three Transactions of the American Mathematical Society 353 (2001), 3267-3305

[77] Z. Hou, Local complex singularity exponents for isolated singularities Ph.D. Thesis, Massachusetts Institute of Technology, 2004

[78] J.-M. Hwang, Log canonical thresholds of divisors on Grassmannians Mathematische Annalen 334 (2006), 413-418

[79] J.-M. Hwang, Log canonical thresholds of divisors on Fano manifolds of Picard rank 1 Compositio Mathematica 143 (2007), 89-94

[80] K. Hulek, Stable rank-2 vector bundles on $\mathbb{P}^{2}$ with $c_{1}$ odd Mathematische Annalen 242 (1979), 241-266

[81] J. Johnson, J. Kollár, Kähler-Einstein metrics on log del Pezzo surfaces in weighted projective 3-spaces Annales de l'Institut Fourier 51 (2001), 69-79

[82] J. Johnson, J. Kollár, Fano hypersurfaces in weighted projective 4-spaces Experimental Mathematics 10 (2001), 151-158 
[83] A. J. de Jong, N. Shepherd-Barron, A. V.de Ven, On the Burkhardt quartic Mathematische Annalen 286 (1990), 309-328

[84] D. Jaffe, D. Ruberman, A sextic surface cannot have 66 nodes Journal of Algebraic Geometry 6 (1997), 151-168

[85] P. Jahnke, T. Peternell, Almost del Pezzo manifolds arXiv:math/0612516 (2006)

[86] P. Jahnke, T. Peternell, I. Radloff Threefolds with big and nef anticanonical bundles II arXiv:0710.2763 (2007)

[87] P. Jahnke, I. Radloff, Fano threefolds with sections in $\Omega_{V}^{1}(1)$ Mathematische Nachrichten 280 (2007), 127-139

[88] Th. Jeffres, Singular set of some Kähler orbifolds Transactions of the American Mathematical Society 349 (1997), 1961-1971

[89] A. R. Iano-Fletcher, Working with weighted complete intersections L.M.S. Lecture Note Series 281 (2000), 101-173

[90] J. Igusa, On the first terms of certain asymptotic expansions Complex analysis and algebraic geometry Iwanami Shoten, Tokyo (1977), 357-368

[91] A. Iliev, C. Schuhmann, Tangent scrolls in prime Fano threefolds Kodai Mathematical Journal 23 (2000), 411-431

[92] V.Iskovskikh, Fano 3-folds I Mathematics of the USSR, Izvestija 11 (1977), 485-527

[93] V. Iskovskikh, Fano 3-folds II Mathematics of the USSR, Izvestija 12 (1978), 469-506

[94] V. Iskovskikh, Birational automorphisms of three-dimensional algebraic varieties Journal of Soviet Mathematics 13 (1980), 815-868

[95] V. Iskovskikh, Factorization of birational maps of rational surfaces from the viewpoint of Mori theory Russian Mathematical Surveys 51 (1996), 585-652

[96] V. Iskovskikh, Birational rigidity of Fano hypersurfaces in the framework of Mori theory Russian Mathematical Surveys 56 (2001), 207-291

[97] V. Iskovskikh, Yu. Manin, Three-dimensional quartics and counterexamples to the Lüroth problem Mathematics of the USSR-Sbornik 15 (1971), 140-166

[98] V. Iskovskikh, Yu. Prokhorov, Fano varieties Encyclopaedia of Mathematical Sciences 47 (1999) Springer, Berlin

[99] V. Iskovskikh, A. Pukhlikov, Birational automorphisms of multidimensional algebraic manifolds Journal of Mathematical Sciences 82 (1996), 3528-3613

[100] A.-S. Kaloghiros, The topology of terminal quartic 3-folds arXiv:math/0707.1852 (2007)

[101] A.-S. Kaloghiros, The defect of Fano 3-folds arXiv:math/0711.2186 (2007)

[102] Y. Kawamata, K. Matsuda, K. Matsuki, Introduction to the minimal model problem Advanced Studies in Pure Mathematics 10 (1987), 283-360

[103] H. Kim, Y. Lee, Log canonical thresholds of semistable plane curves Mathematical Proceedings of the Cambridge Philosophical Society 137 (2004), 273-280

[104] J. Kollár, Log surfaces of general type: some conjectures Contemporary Mathematics 162 (1994), 261-275

[105] J. Kollár, Singularities of pairs Proceedings of Symposia in Pure Mathematics 62 (1997), 221-287

[106] J. Kollár, Which powers of holomorphic functions are integrable? arXiv:0805.0756 (2008)

[107] J. Kollár, Universal untwisting of birational maps in preparation (2008)

[108] J. Kollár, S. Mori, Birational geometry of algebraic varieties Cambridge University Press (1998)

[109] T. Kuwata, On log canonical thresholds of reducible plane curves American Journal of Mathematics 121 (1999), 701-721

[110] T. Kuwata, On log canonical thresholds of surfaces in $\mathbb{C}^{3}$ Tokyo Journal of Mathematics 22 (1999), 245-251

[111] R. Lazarsfeld, Positivity in algebraic geometry II Springer-Verlag, Berlin, 2004 
[112] Y. Lee, Chow stability criterion in terms of log canonical threshold Journal of the Korean Mathematical Society 45 (2008), 467-477

[113] Zh. Lu, On the Futaki invariants of complete intersections Duke Mathematical Journal 100 (1999), 359-372

[114] M. Lübke, Stability of Einstein-Hermitian vector bundles Manuscripta Mathematica 42 (1983), 245-257

[115] T. Mabuchi, Einstein-Kähler forms, Futaki invariants and convex geometry on toric Fano varieties Osaka Journal of Mathematics 24 (1987), 705-737

[116] T. Mabuchi, S. Mukai, Stability and Einstein-Kähler metric of a quartic del Pezzo surface Lecture Notes in Pure and Applied Mathematics 145 (1993), 133-160

[117] Yu. Manin, Rational surfaces over perfect fields Publications Mathematiques, Institut des Hautes Etudes Scientifiques 30 (1966), 55-97

[118] Yu. Manin, Rational surfaces over perfect fields, II Mathematics of the USSR, Sbornik 1 (1967), 141-168

[119] Yu. Manin, New dimensions in geometry Russian Mathematical Surveys 39 (1984), 51-83

[120] M. Marchisio, Unirational quartic hypersurfaces Bollettino della Unione Matematica Italiana 8 (2000), 301-313

[121] K. Matsuki, Introduction to the Mori program Universitext, Springer, 2002.

[122] Y.Matsushima, Sur la structure du groupe d'homéomorphismes analytiques d'une certaine variété kählérienne Nagoya Mathematical Journal 11 (1957), 145-150

[123] H. Matsumura, P. Monsky, On the automorphisms of hypersurfaces Journal of Mathematics of Kyoto University 3 (1964), 347-361

[124] J. McKernan, Yu. Prokhorov, Threefold thresholds Manuscripta Mathematica 114 (2004), 281-304

[125] M. Mella, Birational geometry of quartic 3-folds II: the importance of being $\mathbb{Q}$-factorial Mathematische Annalen 330 (2004), 107-126

[126] S. Mori, S. Mukai, Classification of Fano 3-folds with $B_{2} \geqslant 2$ Manuscripta Mathematica 36 (1981), 147-162

[127] S. Mori, S. Mukai, Classification of Fano 3-folds with $B_{2} \geqslant 2$. Erratum Manuscripta Mathematica 110 (2003), 407

[128] S. Mori, S. Mukai, On Fano 3-folds with $B_{2} \geqslant 2$ Advanced Studies in Pure Mathematics, Algebraic Varieties and Analityc Varieties 1 (1983), 101-129

[129] S. Mori, S. Mukai, Classification of Fano 3-folds with $B_{2} \geqslant 2, I$ Algebraic and Topological Theories - to the memory of Dr. Takehiko Miyata, Kinokuniya (1985), 496-545

[130] S. Mukai, H. Umemura, Minimal rational threefolds Lecture Notes in Mathematics 1016 (1983), 490-518

[131] M. Mustata, Singularities of pairs via jet schemes Journal of the American Mathematical Society 15 (2002) 599-615

[132] A. Nadel, Multiplier ideal sheaves and Kähler-Einstein metrics of positive scalar curvature Annals of Mathematics 132 (1990), 549-596

[133] B. Nill, Complete toric varieties with reductive automorphism group Mathematische Zeitschrift 252 (2006), 767-786

[134] T. Ohsawa and K. Takegoshi, On the extension of $L^{2}$ holomorphic functions Mathematische Zeitschrift 195 (1987) 197-204

[135] T. Ohsawa, On the extension of $L^{2}$ holomorphic functions, II Publications of the Research Institute for Mathematical Sciences, Kyoto University 24 (1988), 265-275

[136] J. Park, Birational maps of del Pezzo fibrations Journal fur die Reine und Angewandte Mathematik 538 (2001), 213-221

[137] J. Park, A note on del Pezzo fibrations of degree 1 Communications in Algebra 31 (2003), 5755-5768

[138] Th. Peternell, M. Schneider, Compactifications of $\mathbb{C}^{3} . I$ Mathematische Annalen 280 (1988), 129-146

[139] Th. Peternell, Compactifications of $\mathbb{C}^{3}$. II Mathematische Annalen 283 (1989), 121-137

[140] K. Pettersen, On nodal determinantal quartic hypersurfaces in $\mathbb{P}^{4}$ Ph.D. Thesis, University of Oslo, 1998 
[141] D. Phong, J. Sturm, On a conjecture of Demailly and Kollár Asian Journal of Mathematics 4 (2000), 221-226

[142] Yu. Prokhorov, Automorphism groups of Fano 3-folds Russian Mathematical Surveys 45 (1990), 222-223

[143] Yu. Prokhorov, Exotic Fano varieties Moscow University Mathematical Bulletin 45 (1990), 36-38

[144] Yu. Prokhorov, Fano threefolds of genus 12 and compactifications of $\mathbb{C}^{3}$ Saint Petersburg Mathematical Journal 3 (1992), 855-864

[145] Yu. Prokhorov, On log canonical thresholds Communications in Algebra 29 (2001), 3961-3970

[146] Yu. Prokhorov, On log canonical thresholds. II Communications in Algebra 30 (2002), 5809-5823

[147] A. Pukhlikov, Birational isomorphisms of four-dimensional quintics Inventiones Mathematicae 87 (1987), 303-329

[148] A. Pukhlikov, Birational automorphisms of a double space and double quadric Mathematics of the USSR, Izvestija 32 (1989), 233-243

[149] A. Pukhlikov, Birational automorphisms of a three-dimensional quartic with a simple singularity Matematicheskii Sbornik 177 (1988), 472-496

[150] A.Pukhlikov, Notes on theorem of V.A.Iskovskikh and Yu.I.Manin about threefold quartic Proceedings of Steklov Institute 208 (1995), 278-289.

[151] A.Pukhlikov, Birational automorphisms of Fano hypersurfaces Inventiones Mathematicae 134 (1998), 401-426

[152] A.Pukhlikov, Fiberwise birational correspondences Mathematical Notes 68 (2000), 102-112

[153] A.Pukhlikov, Birationally rigid double Fano hypersurfaces Sbornik: Mathematics 191 (2000), 883-908

[154] A. Pukhlikov, Birationally rigid Fano complete intersections Journal fur die Reine und Angewandte Mathematik 541 (2001), 55-79

[155] A. Pukhlikov, Birationally rigid Fano hypersurfaces Izvestiya: Mathematics 66 (2002), 1243-1269

[156] A. Pukhlikov, Birationally rigid Fano hypersurfaces with isolated singularities Sbornik: Mathematics 193 (2002), 445-471

[157] A. Pukhlikov, Birational geometry of Fano direct products Izvestiya: Mathematics 69 (2005), 1225-1255

[158] A. Pukhlikov, Birational geometry of algebraic varieties with a pencil of Fano complete intersections Manuscripta Mathematica 121 (2006), 491-526

[159] A. Pukhlikov, Birationally rigid varieties. I: Fano varieties Russian Mathematical Surveys 62 (2007), 857-942

[160] A. Pukhlikov, Explicit examples of birationally rigid Fano varieties Moscow Mathematical Journal 7 (2007), 543-560

[161] J. Rauschning, P. Slodowy, An aspect of icosahedral symmetry Canadian Mathematical Bulletin 45 (2005), 686-696

[162] J. Ross, R. Thomas, An obstruction to the existence of constant scalar curvature Kähler metrics Journal of Differential Geometry 72 (2006), 429-466

[163] J. Ross, R. Thomas, A study of the Hilbert-Mumford criterion for the stability of projective varieties Journal of Algebraic Geometry 16 (2007), 201-255

[164] D. Ryder, Classification of elliptic and K3 fibrations birational to some $\mathbb{Q}$-Fano 3 -folds Journal of Mathematical Sciences of the University of Tokyo 13 (2006), 13-42

[165] D. Ryder, The Curve Exclusion Theorem for elliptic and K3 fibrations birational to Fano 3-fold hypersurfaces arXiv:math.AG/0606177 (2006)

[166] V.Sarkisov, Birational automorphisms of conic bundles Izvestiya Akademii Nauk SSSR, Seriya Matematicheskaya 44 (1980), 918-945

[167] F.-O. Schreyer, Geometry and algebra of prime Fano 3-folds of genus 12 Compositio Mathematica 127 (2001), 297-319

[168] V.Shokurov, The existence of a line on Fano varieties Izvestiya Akademii Nauk SSSR. Seriya Matematicheskaya 43 (1979), 922-964

[169] V.Shokurov, Three-fold log flips Russian Academy of Sciences, Izvestiya Mathematics 40 (1993), 95-202 
[170] V.Shokurov, On rational connectedness Mathematical Notes 68 (2000), 771-782

[171] C. Shramov, $\mathbb{Q}$-factorial quartic threefolds Sbornik: Mathematics 198 (2007), 1165-1174

[172] C.Shramov, Birational automorphisms of nodal quartic threefolds arXiv:0803.4348 (2008)

[173] Y.T.Siu, The existence of Kähler-Einstein metrics on manifolds with positive anticanonical line bundle and a suitable finite symmetry group Annals of Mathematics 127 (1988), 585-627

[174] J. Song, The $\alpha$-invariant on toric Fano threefolds American Journal of Mathematics 127 (2005), 1247-1259

[175] J.Sparks, New results in Sasaki-Einstein geometry arXiv:math/0701518 (2007)

[176] A.Steffens, On the stability of the tangent bundle of Fano manifolds Mathematische Annalen 304 (1996), 635-643

[177] M. Szurek, J. Wiśniewski, Fano bundles of rank 2 on surfaces Compositio Mathematica 76 (1990), 295-305

[178] K. Takeuchi, Some birational maps of Fano 3-folds Compositio Mathematica 71 (1989), 265-283

[179] G. Tian, On Kähler-Einstein metrics on certain Kähler manifolds with $c_{1}(M)>0$ Inventiones Mathematicae 89 (1987), 225-246

[180] G. Tian, On Calabi's conjecture for complex surfaces with positive first Chern class Inventiones Mathematicae 101 (1990), 101-172

[181] G. Tian, On a set of polarized Kähler metrics on algebraic manifolds Journal of Differential Geometry 32 (1990), 99-130

[182] G. Tian, Kähler-Einstein metrics with positive scalar curvature Inventiones Mathematicae 130 (1997), 1-37

[183] G. Tian, S. T. Yau, Kähler-Einstein metrics on complex surfaces with $C_{1}>0$ Communications in Mathematical Physics 112 (1987), 175-203

[184] A. Varchenko, Complex exponents of a singularity do not change along the stratum $\mu=$ constant Functional Analysis and Its Applications 16 (1982), 1-9

[185] A. Varchenko, Semi-continuity of the complex singularity index Functional Analysis and Its Applications 17 (1983), 307-308

[186] A. Varchenko, On semicontinuity of spectrum and upper bound for number of singular points of hypersurfaces Doklady Akademii Nauk SSSR 270 (1983), 1294-1297

[187] X. Wang, X. Zhu, Kähler-Ricci solitons on toric manifolds with positive first Chern class Advances in Mathematics 188 (2004), 87-103

[188] J. Wahl, Nodes on sextic hypersurfaces in $\mathbb{P}^{3}$ Journal of Differential Geometry 48 (1998), 439-444

[189] J. Won, Anticanonical divisors on Gorenstein del Pezzo surfaces Master Thesis, POSTECH, 2004

[190] S. T. Yau, On the Ricci curvature of a compact Kähler manifold and the complex Monge-Ampère equation, I Communications on Pure and Applied Mathematics 31 (1978), 339-411

[191] S. T. Yau, Review on Kähler-Einstein metrics in algebraic geometry Israel Mathematical Conference Proceedings 9 (1996), 433-443

[192] A. Zagorskii, Three-dimensional conical fibrations Mathematical Notes 21 (1977), 420-427

[193] Q. Zhang, Rational connectedness of $\log \mathbb{Q}$-Fano varietiess Journal fur die Reine und Angewandte Mathematik 590 (2006), 131-142

University of Edinburgh, Kings Buildings, Mayfield Road, Edinburgh EH9 3JZ, UK

E-mail address: cheltsov@yahoo.com, shramov@mccme.ru 